\title{
Identification of genes regulated in target organs of acute graft versus host disease in different organs in different species
}

\section{Doctoral Thesis}

\author{
In partial fulfillment of the requirements for the degree \\ "Doctor rerum naturalium (Dr. rer. nat.)" \\ in the Molecular Medicine Study Program \\ at the Georg-August University Göttingen
}

submitted by

Pranali Nitin Shah

born in

Gujarat, India

Göttingen, June 2016 


\section{Members of the thesis committee}

Supervisor and first member of the thesis committee:

Prof. Dr. med. Ralf Dressel

Department of Cellular and Molecular Immunology

University Medical Center

Georg-August University Göttingen

Second member of the thesis committee:

Prof. Dr. rer. nat. Lutz Walter

Department of Primate Genetics

German Primate Center

Third member of the thesis committee:

Prof. Dr. rer. nat. Holger Reichardt

Department of Cellular and Molecular Immunology

University Medical Center

Georg-August University Göttingen

Date of Disputation: 19.08.2016 


\section{AFFIDAVIT}

Here I declare that my doctoral thesis entitled

"Identification of genes regulated in target organs of acute graft versus host disease in different organs in different species"

has been written independently with no other sources and aids than quoted.

Date

Signature 



\section{Table of contents}

Acknowledgements ............................................................................................................................

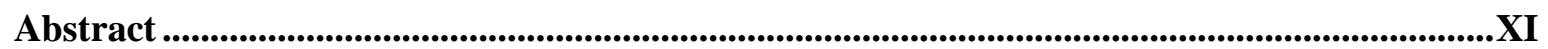

List of figures ................................................................................................................III

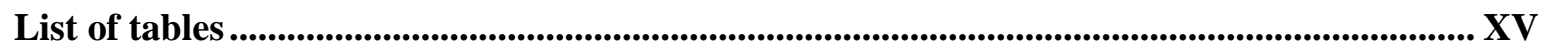

List of abbreviations.................................................................................................... XVII

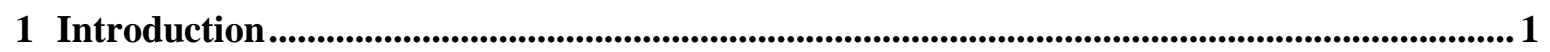

1.1 Allogeneic hematopoietic stem cell transplantation ......................................................... 1

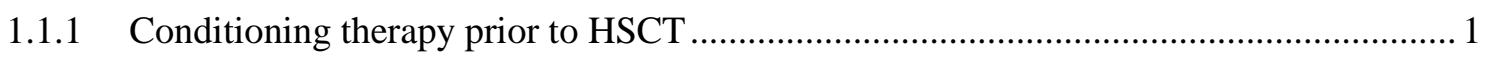

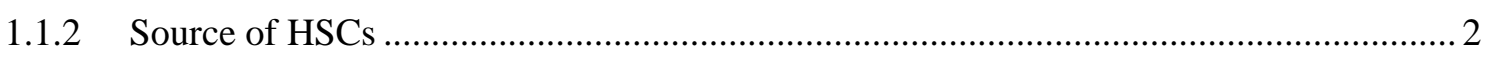

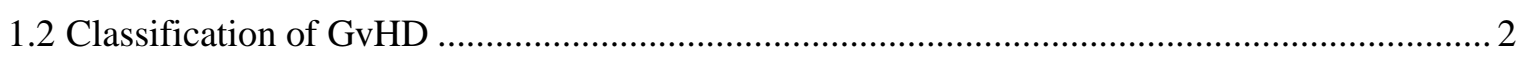

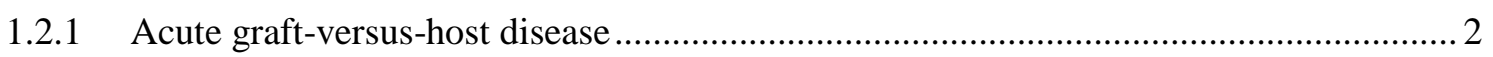

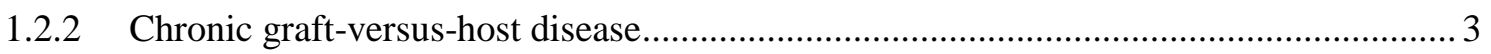

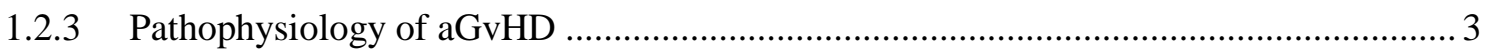

1.2.4 Histopathological manifestation of GvHD in target organs .......................................... 7

1.3 Important HLA and non-HLA related risk factors involved in aGvHD .................................. 8

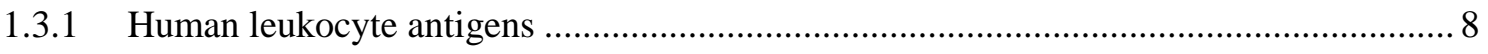

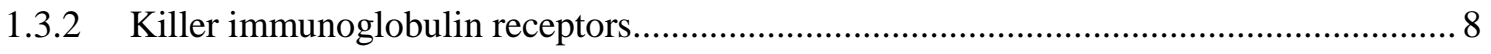

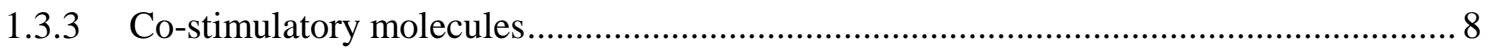

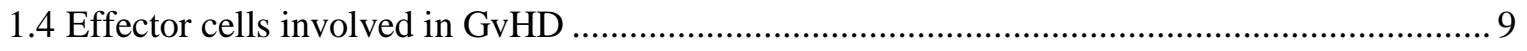

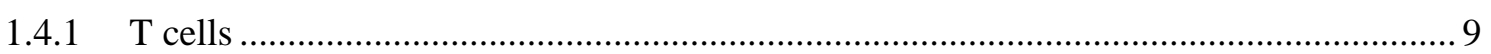

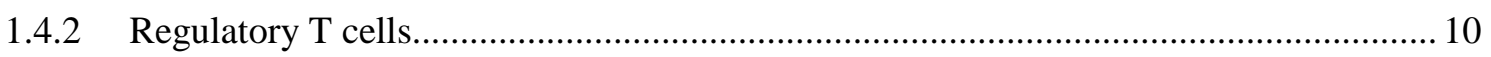

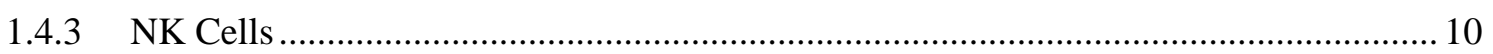

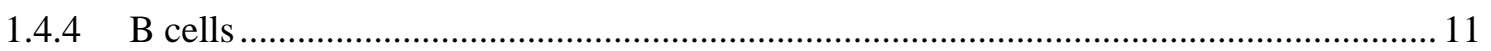

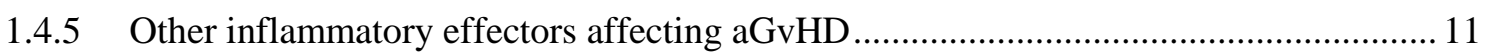

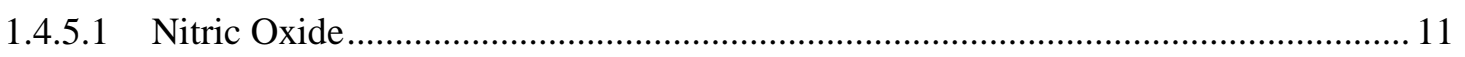

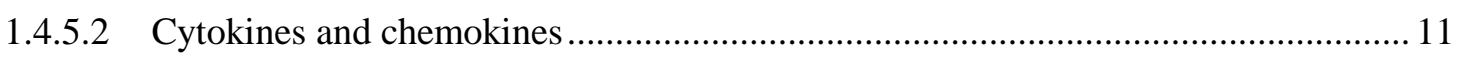

1.4.5.2.1 Regulation of cytokine genes in aGvHD ........................................................... 12

1.4.5.2.2 Regulation of chemokine genes in aGvHD ......................................................... 13

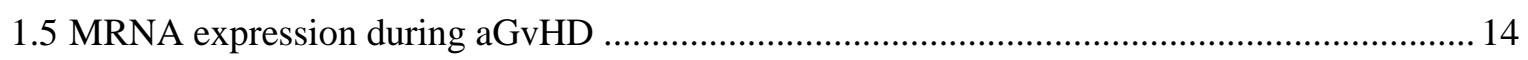

1.5.1 Expression of previously identified candidate genes in rat skin and clinical

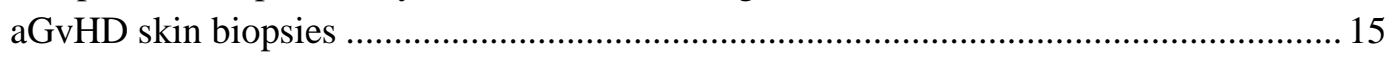

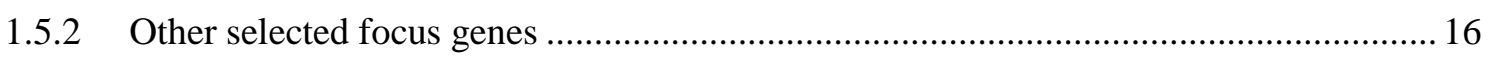

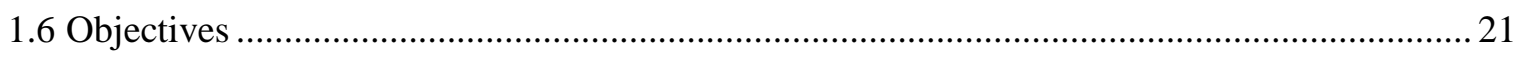

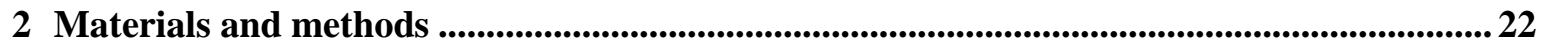

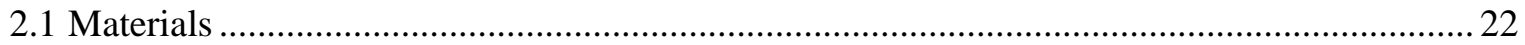




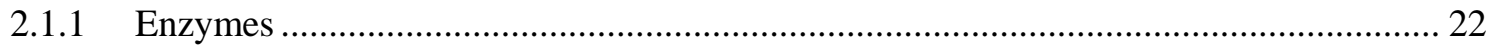

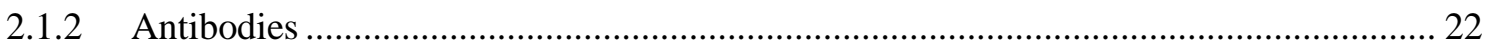

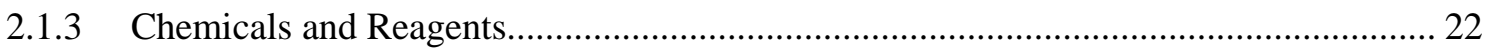

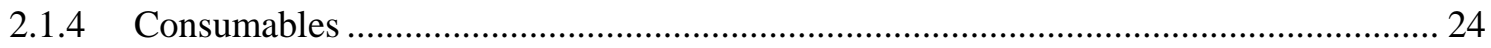

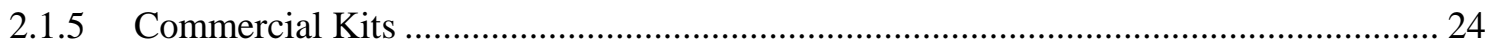

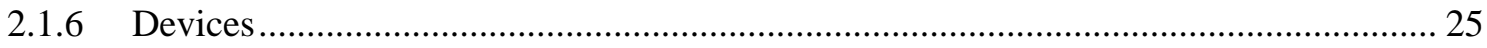

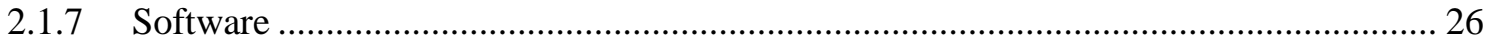

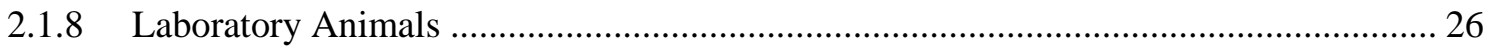

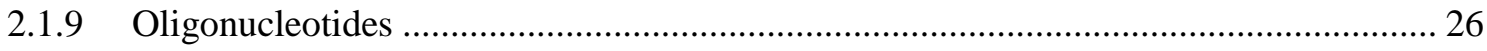

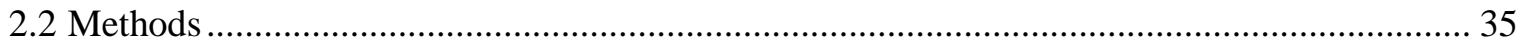

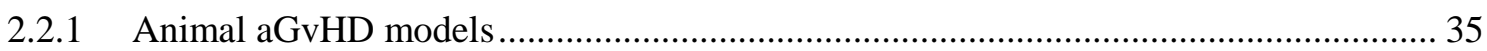

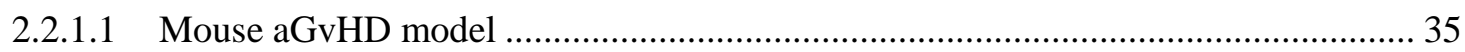

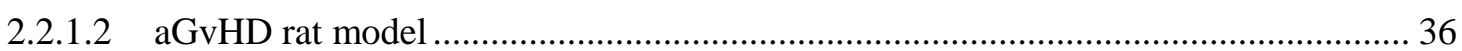

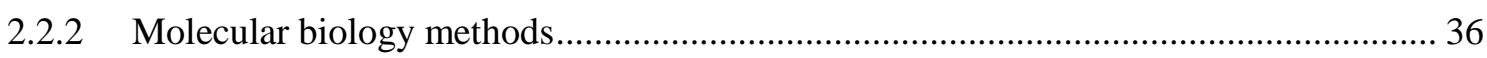

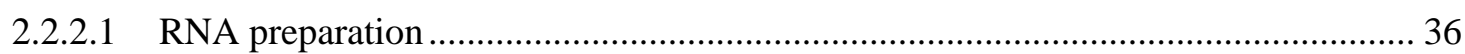

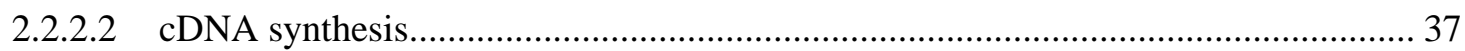

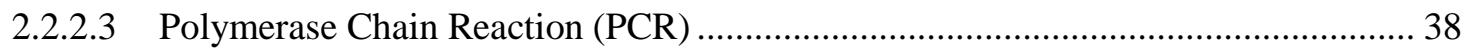

2.2.2.4 Quantitative real-time PCR (qRT-PCR) ….......................................................... 39

2.2.3 Gene expression using Biomark Fluidigm dynamic Arrays .......................................... 40

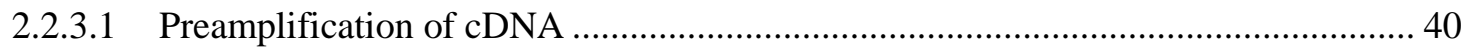

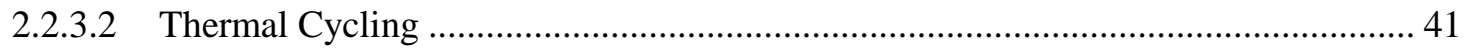

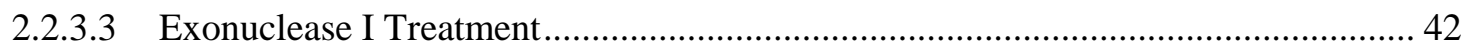

2.2.3.4 Priming and Loading the Dynamic Array IFC ........................................................... 44

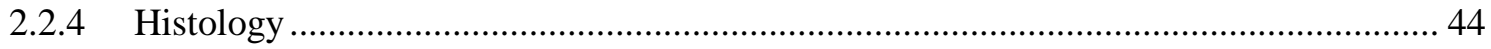

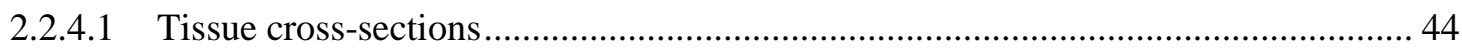

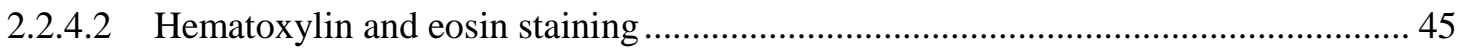

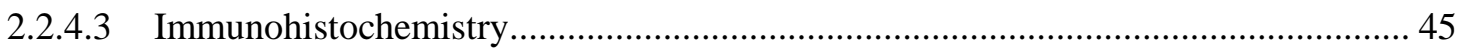

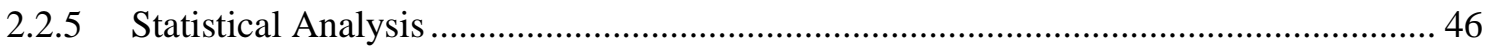

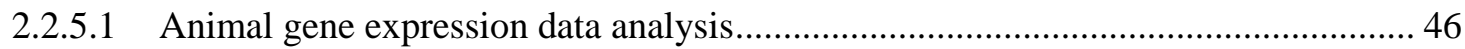

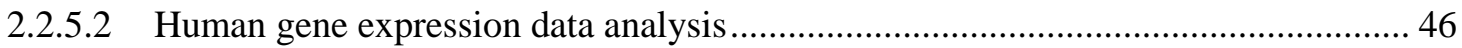

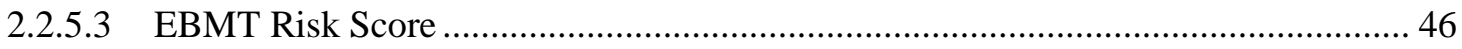

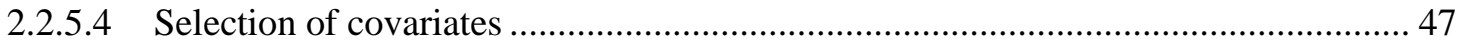

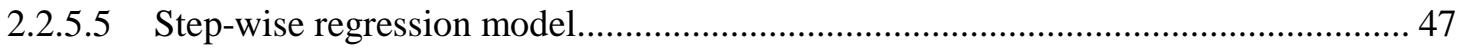

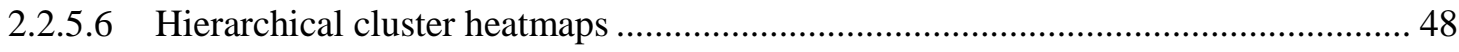

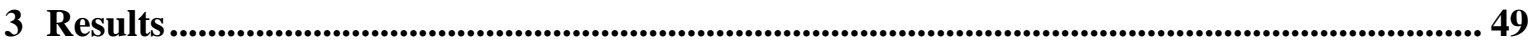

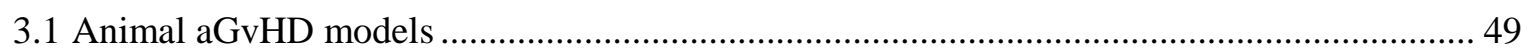




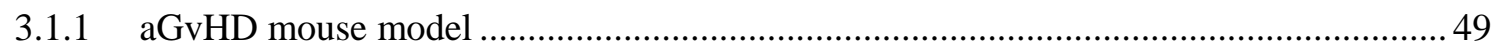

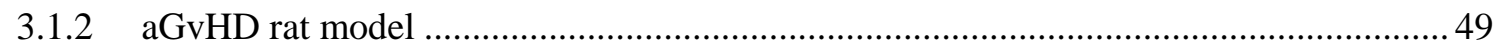

3.1.3 Gene expression profiling in different aGvHD tissues................................................ 50

3.2 Important genes regulated in different mouse tissues due to preconditioning and aGvHD...... 50

3.2.1 Expression of cytokines associated with Th1 responses in mice ................................... 51

3.2.2 Expression of cytokines associated with $\mathrm{Th} 2$ responses in mice …............................... 53

3.2.3 Expression of cytokines associated with Th17 responses in mice ..................................55

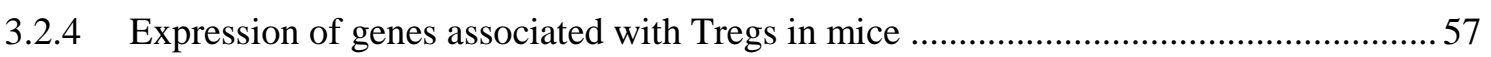

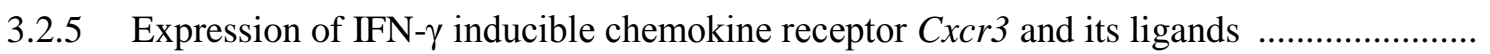

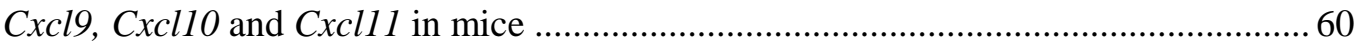

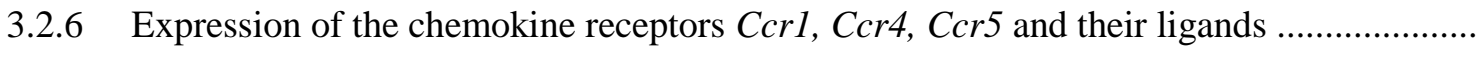

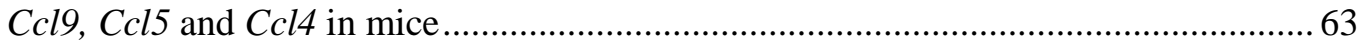

3.2.7 Expression of Cxcl15, Cxcl16 and chemokine receptor Cxcr4 in mice.......................... 65

3.2.8 Expression of the chemokine receptor $\mathrm{Cx} 3 \mathrm{crl}$ and its ligand $\mathrm{Cx} 3 \mathrm{cll}$ in mice ............... 67

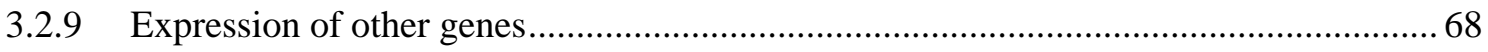

3.3 Gene expression of previously identified candidate genes in mice and rat aGvHD ................. 71

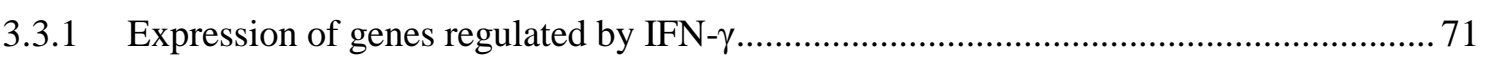

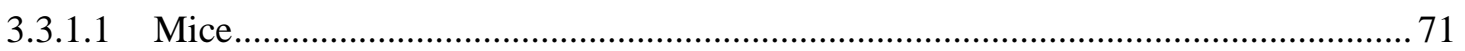

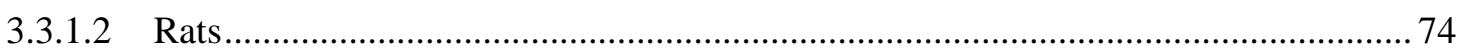

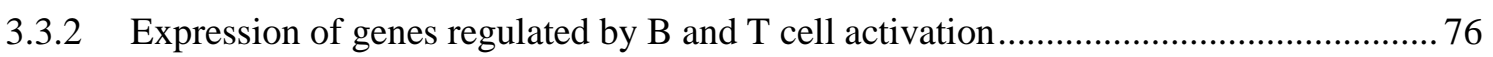

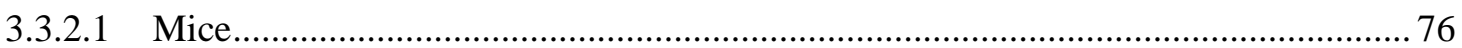

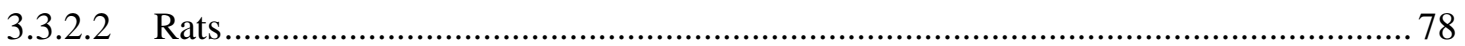

3.3.3 Expression of genes regulated by innate immune responses......................................... 80

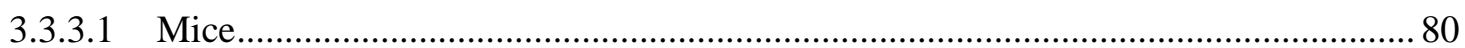

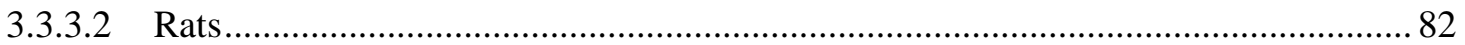

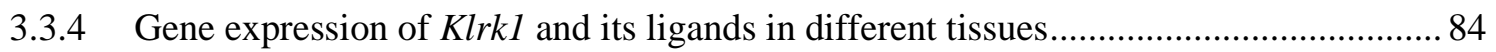

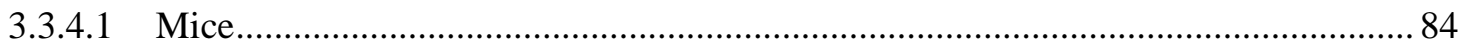

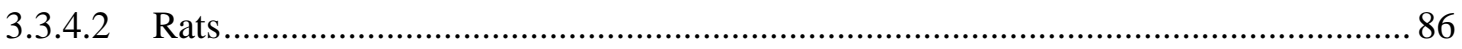

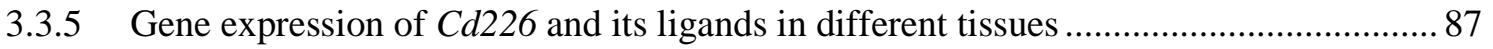

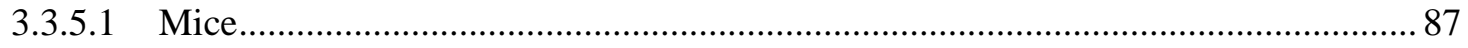

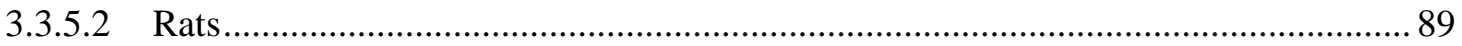

3.4 $\mathrm{T}$ cell infiltration in the small and large intestines during aGvHD .......................................... 90

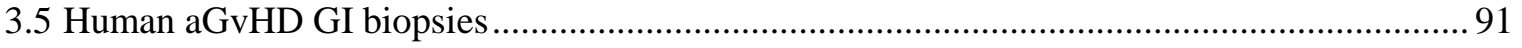

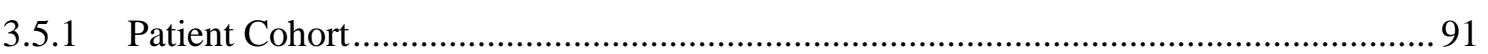

3.5.2 Gene expression analysis during GI aGvHD in all patients .......................................... 93

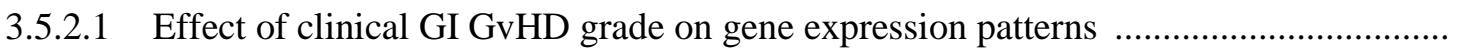

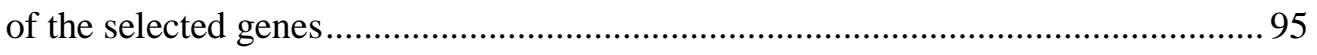


3.5.2.2 Effect of histological aGvHD score on gene expression patterns

of the selected genes

3.5.2.2.1 Histological aGvHD grades 1-4 versus no aGvHD 97

3.5.2.2.2 Histological aGvHD grades 2-4 versus no or low aGvHD (grades 0-1). 98

3.5.2.3 Effect of gene regulation on transplant related mortality (TRM) in HSCT patients

3.5.2.4 Effect of time period after transplant on gene expression patterns of the selected genes

3.5.3 Gene expression in patients with no steroid ${ }^{*}$ treatment 100

3.5.3.1 Effect of clinical GI GvHD grade on gene expression patterns of the selected genes in patients with no steroid treatment ${ }^{*}$.

3.5.3.2 Effect of histological aGvHD score on gene expression patterns of the selected genes in patients with no steroid treatment ${ }^{*}$.

3.5.3.2.1 Histological aGvHD grades 2-4 versus no or low aGvHD (grades 0-1).

3.5.3.3 Effect of gene regulation on transplant related mortality (TRM) in HSCT patients with no steroid treatment ${ }^{*}$.

3.5.4 Regulation of NK receptors and their ligands during clinical GI aGvHD 104

3.5.4.1 Regulation of KLRKI and its ligands in clinical GI aGvHD . 105

3.5.4.2 Regulation of $C D 226$ and its ligands in clinical GI aGvHD 105

3.5.4.3 Effect of regulation of KLRK1, CD226 and their ligands on TRM in HSCT patients with clinical GI aGvHD

3.5.4.4 Effect of steroids on the regulation of KLRK1, CD226 and their ligands in clinical GI aGvHD

4 Discussion

4.1 Comparison of gene expression in different target organs of the same species

4.1.1 Comparison of genes associated with different immune responses in the different aGvHD mouse target tissues compared to healthy controls

4.1.2 Comparison of previously identified candidate genes in the different aGvHD target tissues in mouse and rats

4.2 Differences in gene expression due to conditioning... 118

4.3 Comparison of gene expression in different target organs in different species.................... 123

4.3.1 Comparison of gene expression between mouse and rat aGvHD

4.3.2 Comparison of gene expression between mouse intestinal and human GI GvHD

4.3.3 Comparison of gene expression between mouse, rat aGvHD in small intestine..... and human GI GvHD

4.4 Gene expression pattern of KLRKI and its ligands in aGvHD tissues in different species.... 128

4.5 Gene expression pattern of $C D 226$ and its ligands in aGvHD tissues in different species .... 130

4.6 Gene expression in human GI aGvHD biopsies 
5 Summary and conclusions ........................................................................................................... 132

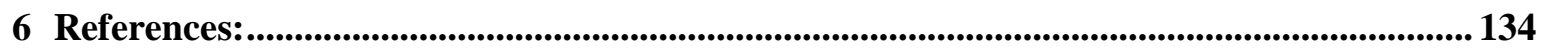

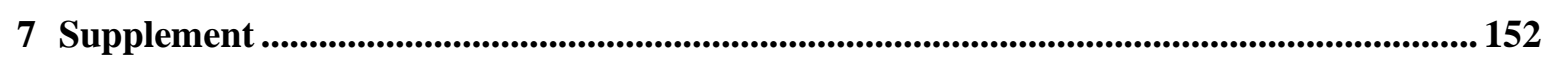

7.1 Expression of genes in aGvHD mice compared to healthy controls in different tissues ........ 152

7.2 Expression of genes in aGvHD mice compared to BM controls in different tissues............... 154

7.3 Expression of genes in BM mice compared to healthy controls in different tissues ............... 156

7.4 Expression of genes in aGvHD rat compared to syngeneic rats in different tissues............... 158

Curriculum vitae ...................................................................................................................................................... 159 


\section{Acknowledgements}

First and foremost, I would like to thank Prof. Ralf Dressel for giving me the opportunity to carry out my PhD studies in his research group. I sincerely thank you for your guidance and support in helping me evolve into an independent researcher.

I also want to thank Prof. Lutz Walter from the Department of Primate Genetics of the German Primate Center (DPZ) Göttingen for supervision of my work and guidance, being part of my thesis committee, and for all the work conducted at your facility to perform my experiments. My sincere thanks to Prof. Holger Reichardt from the Department of Cellular and Molecular Immunology, UMG, Göttingen, for all your suggestions and guidance during the thesis committees and throughout the project, and for providing me with my mice samples.

For help with the mice work, I would like to thank Dr. Henrike Fischer and Dr. Katharina Jörß. I especially want to thank Dr. Olena Zaitseva for all the time spent helping me with my experiments.

To our technician Leslie Elsner, I want to express my gratitude for your assistance. In addition, I want to thank all my colleagues, especially Carina Hamann, Kanika Vanshylla and Dr. Sebastian Monecke for making these last three years memorable.

I would also like to thank my entire Celleurope team, for giving me an invaluable experience being part of such an enthusiastic research team. I especially want to thank Prof. Anne Dickinson, from Newcastle University, UK and Prof. Bent Rolstad and Dr. Marit Inngjerdingen from University of Oslo, Norway, for hosting me during my secondments.

In addition, I want to thank Prof. Ernst Holler, for providing me with the invaluable human clinical biopsies. 
Moreover, I want to express my gratitude to all my colleagues in this project, for making these last three years an incredible experience. A special thanks goes out to Sakhila Ghimire and Margherita Boieri for all your help throughout this project.

A big thanks to Dr. Dörthe Malzahn, from the Department of Genetic Epidemiology of the UMG for your indispensable help with my statistics analyses.

In addition, I owe thanks to the Head of the Institute for Cellular and Molecular Immunology of the UMG Prof. Jürgen Wienands and the secretaries Ingrid Teuteberg and Anika Schindler.

Finally, I would like to thank my family and my friends for their unconditional support. 


\begin{abstract}
Major histocompatibility complex (MHC) matching is essential to reduce the risk of graft versus host disease (GvHD) but minor histocompatibility antigens (mHags) also affect the outcome of hematopoietic stem cell transplantation (HSCT). In addition, single nucleotide polymorphisms (SNPs), which do not give rise to mHags, can influence the result of HSCT. In addition to genotyping, profiling of gene expression in tissues affected by GvHD might identify genes, which are important for the development of GvHD.
\end{abstract}

Previously, 11 non-class I/II MHC and 174 other genes were identified to be regulated during GvH reactions in rat skin explant assays, which provide an in vitro model of GvHD as well as in rat GvHD models. The expression of 27 of these genes was tested in human skin explant assays and in human GvHD skin biopsies and several genes were confirmed to be regulated also in the human skin biopsies. In this study, we aimed to validate the regulation of these candidate genes in tissues affected by acute GvHD (aGvHD) in two animal models and compare their regulation in 186 gastrointestinal biopsies from patients after HSCT. Notably, the candidate genes HCLS1, UBD and TGM2 were strongly upregulated in all the mice aGvHD tissues compared to controls. A similar trend in regulation was observed in the rat aGvHD tissues and human GI GvHD biopsies.

In addition several cytokines, chemokines and other molecules have been implicated in GvHD pathophysiology. In this study we selected several genes that are expected to be important in HSCT outcome in view of previous reports or known functions and determined their regulation in different tissues affected by aGvHD. We wanted to compare the regulation of these genes during aGvHD and after preconditioning and transplantation not leading to aGvHD. In addition, we analysed the regulation of these genes in human GI aGvHD. In the mouse model, an upregulation of Th1, Th2 and Th17 cytokines in the lung, small and large intestines was associated with aGvHD. Genes associated with Treg regulation and activation were increased in the liver, lung and both intestines during aGvHD. Overall, we observed that aGvHD in both the mouse tissues and human GI biopsies was associated with a marked chemokine regulation. Several chemokines $C x c l 9$, Cxcl10, Cxcl11 and their receptor Cxcr3, Ccl4, Ccl9 and their receptors Ccr5, Ccrl were upregulated in mouse tissues affected by aGvHD compared to the healthy controls, whereas a strong downregulation was observed in the expression of the chemokines $\mathrm{Ccl}$, Cx3cll and chemokine receptors $\mathrm{Ccr} 4$ and $\mathrm{Cxcr}$. These genes showed a similar trend of expression in the human GI aGvHD biopsies as well. 
In view of previous data of our group and others, we also studied the gene expression profiles of KLRK1, encoding NKG2D and CD226, encoding DNAM-1, and their ligands in the animal models of aGvHD and human GI GvHD. NKG2D and DNAM-1 are activating NK receptors on NK and $\mathrm{CD}^{+} \mathrm{T}$ cells. They control cytotoxicity and interferon- $\gamma$ production by NK cells and serve as costimulatory molecules on $\mathrm{CD}^{+} \mathrm{T}$ cells. NKG2D and DNAM-1 ligands can be up-regulated in several pathological conditions. Blockade or deficiency of either DNAM-1 or NKG2D in donor cells, has been shown to reduce the intensity of aGvHD in mice. Both NKG2D and DNAM-1 have also been implicated in inducing aGvHD by co-stimulating allogeneic cytotoxic $\mathrm{T}$ cells (CTL) directly via upregulation of their ligands on non-professional antigen presenting cells (APCs). Moreover, we recently described that a polymorphism in the human NKG2D ligand MICA has a major impact on the risk of aGvHD after allogeneic hematopoietic stem cell transplantation (HSCT) by modulating NKG2D signalling. The mRNA expression of KLRK1 and CD226 was increased significantly in all the mouse tissues affected by aGvHD, and showed an increased expression trend in the rat and human GvHD biopsies. Additionally, we observed a strong upregulation of their ligands, Ulbpl, Pvr and Pvrl2 in mouse aGvHD and Raell in the rat. MICA was upregulated in patients with aGvHD that were treated with steroids. On the other hand, Rael was downregulated in the different aGvHD mice tissues and $M I C B$ was downregulated in human GI aGvHD. $P V R$ and $P V R L 2$ showed a trend of upregulation in rats, however they were not differentially regulated in the human aGvHD biopsies. Additionally, the expression of MICA was increased whereas $P V R L 2$ was decreased in patients who died due to transplant related causes compared to patients who were still alive or died due to other causes including relapse of malignancy.

In conclusion, we confirmed that several candidate genes previously suggested to be regulated during aGvHD were indeed significantly regulated in the different tissues in both animal models and human GI biopsies, and a number of genes showed similar expression trends. Moreover, we successfully showed that the regulation of many of our focus genes was significantly altered due to preconditioning, and their regulation was frequently exacerbated due to aGvHD in the different mouse tissues. Conditioning regimens that are less likely to induce these genes could be beneficial for attenuating the aGvHD response. Ligands of the activating NK receptors NKG2D showed a complex expression pattern during GvHD that might be important for the activation of allogeneic CTL in target tissues of aGvHD. 


\section{List of figures}

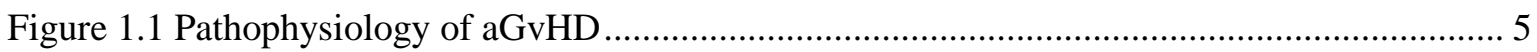

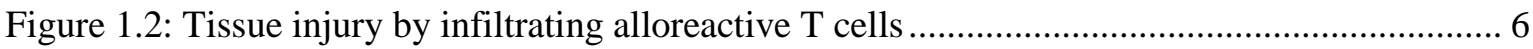

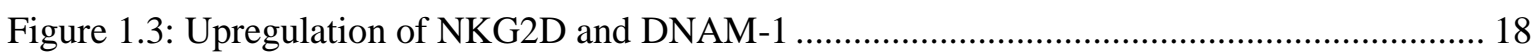

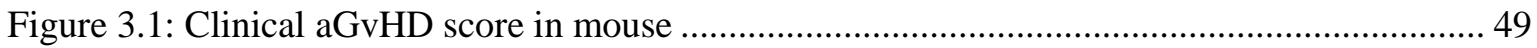

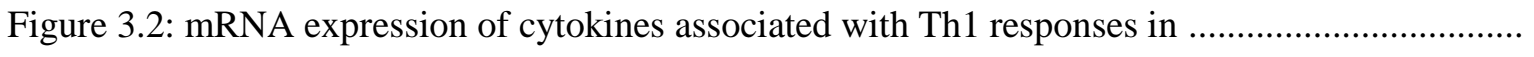

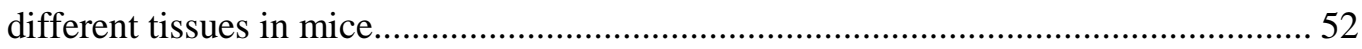

Figure 3.3: mRNA expression of cytokines associated with Th2 responses in

different tissues in mice.

Figure 3.4: mRNA expression of cytokines associated with Th17 responses in

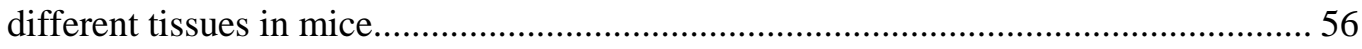

Figure 3.5: mRNA expression of cytokines associated with Tregs responses in

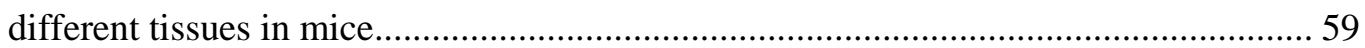

Figure 3.6: mRNA expression of chemokines induced by IFN- $\gamma$ in different tissues in mice......... 62

Figure 3.7: mRNA expression of chemokine receptors $C c r 1, C c r 4, C c r 5$ and ligands

Ccl4, $C c l 5$ and $C c l 9$ in different tissues in mice.

Figure 3.8: mRNA expression of chemokines $C x c l 15, C x c l 16$ and chemokine receptor

Cxcr4 in different tissues in mice

Figure 3.9: mRNA expression of chemokine receptor $C x 3 c r l$ and its ligand $C x 3 c l 1$

in different tissues in mice.

Figure 3.10: mRNA expression other genes involved in immune responses in

different tissues in mice.

Figure 3.11: mRNA expression of candidate genes regulated by IFN- $\gamma$ in

different tissues in mice.

Figure 3.12: mRNA expression of genes regulated by IFN- $\gamma$ in different tissues in rats. 76

Figure 3.13: mRNA expression of candidate genes regulated by activation of

$\mathrm{B}$ and $\mathrm{T}$ cells in different tissues in mice

Figure 3.14: mRNA expression of genes regulated by $\mathrm{B}$ and $\mathrm{T}$ cell activation in

different tissues in rats 79 
Figure 3.15: mRNA expression of candidate genes regulated by innate immune responses in different tissues in mice

Figure 3.16: mRNA expression of genes regulated by innate immune responses in different tissues in rats.

Figure 3.17: mRNA expression of Klrk1 and its ligands in different tissues in mice 85

Figure 3.18: mRNA expression of Klrkl and its ligands in different tissues in rat........................... 86

Figure 3.19: mRNA expression of $C d 226$ and its ligands in different tissues in mice. 88

Figure 3.20: mRNA expression of $\mathrm{Cd} 226$ and its ligands in different tissues in rats. 89

Figure 3.21: CD3 staining of small and large intestines in mice. 91

Figure 3.22: Gene expression of KLRK1 (NKG2D) and its ligands in clinical GI aGvHD biopsies. 105

Figure 3.23: Gene expression of $C D 226$ and its ligands in clinical GI aGvHD biopsies. 105

Figure 3.24: Effect of gene regulation of KLRK1, CD226 and their ligands on TRM in human GI aGvHD.

Figure 3.25: Effect of steroid on gene expression of KLRK1, CD226 and their ligands in human GI aGvHD. 108

Figure 4.1: Hierarchical cluster of genes associated with immune responses in the different tissues of mouse aGvHD compared to healthy controls.

Figure 4.2: Hierarchical cluster of candidate genes in different tissues of mouse and rat aGvHD.

Figure 4.3: Hierarchical clustering of mRNA expression of genes in mouse aGvHD compared to preconditioning, in different organs.

Figure 4.4: Hierarchical clustering of mRNA expression of genes between mouse and rats in different organs.

Figure 4.5: Hierarchical clustering of mRNA expression of genes between human

GI GvHD compared to patients with no GvHD and mouse intestinal aGvHD.

Figure 4.6: Hierarchical clustering of mRNA expression of candidate genes between mice, rat and human GI aGvHD small intestine. 


\section{List of tables}

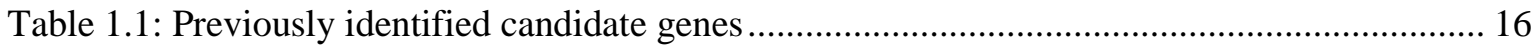

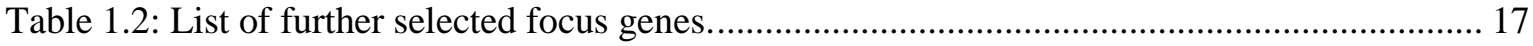

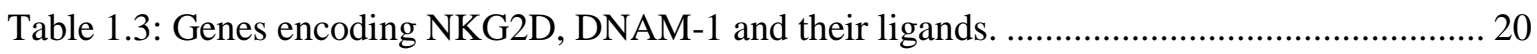

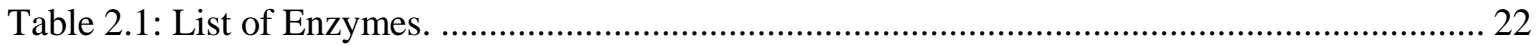

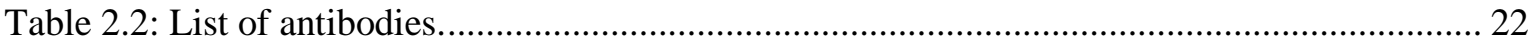

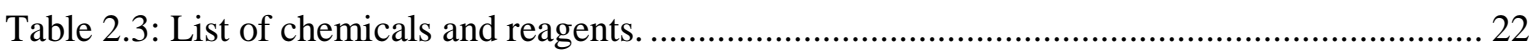

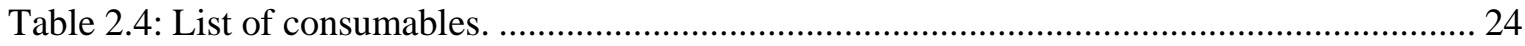

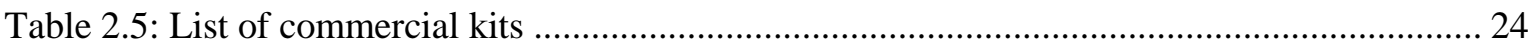

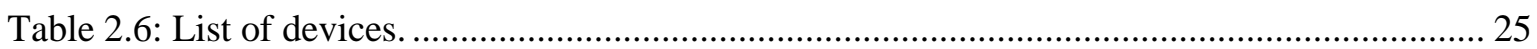

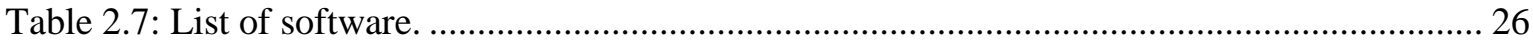

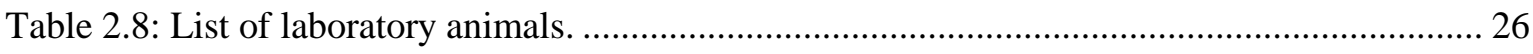

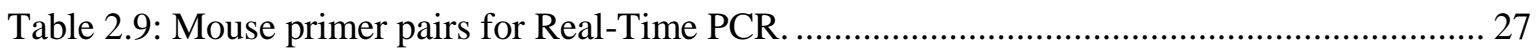

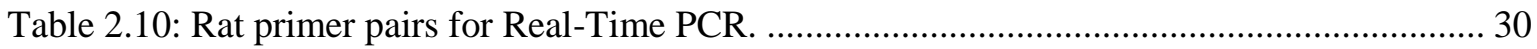

Table 2.11: Human primer pairs for Real-Time PCR. ............................................................... 31

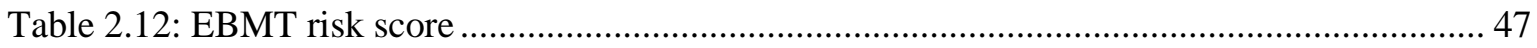

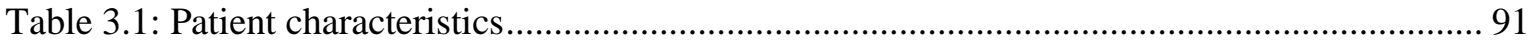

Table 3.2: Genes selected for gene expression study in human GI aGvHD. .................................. 93

Table 3.3: Genes regulated in severe clinical aGvHD versus no aGvHD in patients. ...................... 96

Table 3.4: Genes regulated in histological GI aGvHD (grade 1-4) versus no aGvHD in patients... 97

Table 3.5: Genes regulated in severe histological GI aGvHD (grade 2-4) versus no or low

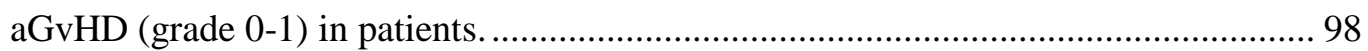

Table 3.6: Effect of gene regulation on TRM in HSCT patients....................................................... 99

Table 3.7: Gene regulation based on time of biopsy taken post transplant................................... 100

Table 3.8: Genes regulated in clinically scored GI aGvHD (grade 1-4) biopsies versus no aGvHD (grade 0 ) in patients with no steroids*

Table 3.9: Genes regulated in severe histological GI aGvHD (grade 2-4) versus no or low aGvHD (grade 0-1) in patients with no steroids*

Table 3.10: Effect of gene regulation on TRM in patients with no steroids. 
Table 3.11: Effect of gene regulation of KLRK1, CD226 and their ligands on TRM in clinical GI aGvHD

Table 3.12: Effect of steroids on the regulation of KLRK1, CD226 and their ligands in clinical GI aGvHD.

Table 4.1: Comparison of candidate genes regulated in previous human and rat skin biopsies and in mouse and rat aGvHD skin in our study.

Table 7.1 Fold changes of regulated genes in aGvHD mice compared to healthy controls in the different tissues

Table 7.2 Fold changes of regulated genes in aGvHD mice compared to BM controls in the different tissues

Table 7.3 Fold changes of regulated genes due to preconditioning and transplantation procedure in BM control mice compared to healthy controls. 156

Table 7.4 Fold changes of regulated genes in aGvHD rats compared to syngeneic rats in different tissues. 


\section{List of abbreviations}

(Mouse and rat genes are denoted in lower case and italics; human genes are denoted in uppercase and italics)

$\begin{array}{ll}\mathrm{y} & \text { year } \\ \gamma & \text { gamma } \\ \mu & \text { micro } \\ - & \text { minus/negative } \\ \% & \text { percentage } \\ { }^{\circ} \mathrm{C} & \text { Celsius } \\ + & \text { plus/positive }\end{array}$

AA Aplastic Anemia

$\mathrm{Ab} \quad$ antibody

aGvHD acute GvHD

ALL

Acute Lymphoblastic Leukemia

AML

Acute Myeloid Leukemia

ANP32A

Acidic nuclear phosphoprotein 32 family member A

APCs

antigen presenting cells

ARG1

Arginase-1

$B A F F$

$\mathrm{B}$ cell activating factor

BCR

B cell receptor

$\mathrm{BM}$

bone marrow

$\mathrm{BMC}$

bone marrow control

BMPR1A

Bone morphogenetic protein receptor type-1A

bp

base pairs

Breg

CIQTNF7

Regulatory B cell

CARD11

$\mathrm{C} 1 \mathrm{q}$ and tumor necrosis factor related protein 7

CCL4

Capsase recruitment domain-containing protein 11

CCL5

Chemokine (C-C) motif ligand 4

Ccl9

Chemokine (C-C) motif ligand 5

Ccrl

Chemokine (C-C) motif ligand 9

CCR4

Chemokine (C-C) motif receptor 1

CCR5

Chemokine (C-C) motif receptor 4

CD154

Chemokine (C-C) motif receptor 5

CD226 (DNAM-1)

Cluster of differentiation 154

CD28

Cluster of differentiation 226

CD40

Cluster of differentiation 28

Cluster of differentiation 40

CD80

Cluster of differentiation 80

CD86

cGvHD

Cluster of differentiation 86

CLL

chronic GvHD

CMV

Chronic Lymphoblastic leukemia

$\mathrm{Ct}$

cytomegalovirus

CTL

CX3CL1

Threshold cycle

cytotoxic T lymphocyte

Chemokine (C-X3-C motif) ligand 1

CX3CR1

Chemokine (C-X3-C motif) receptor 1 


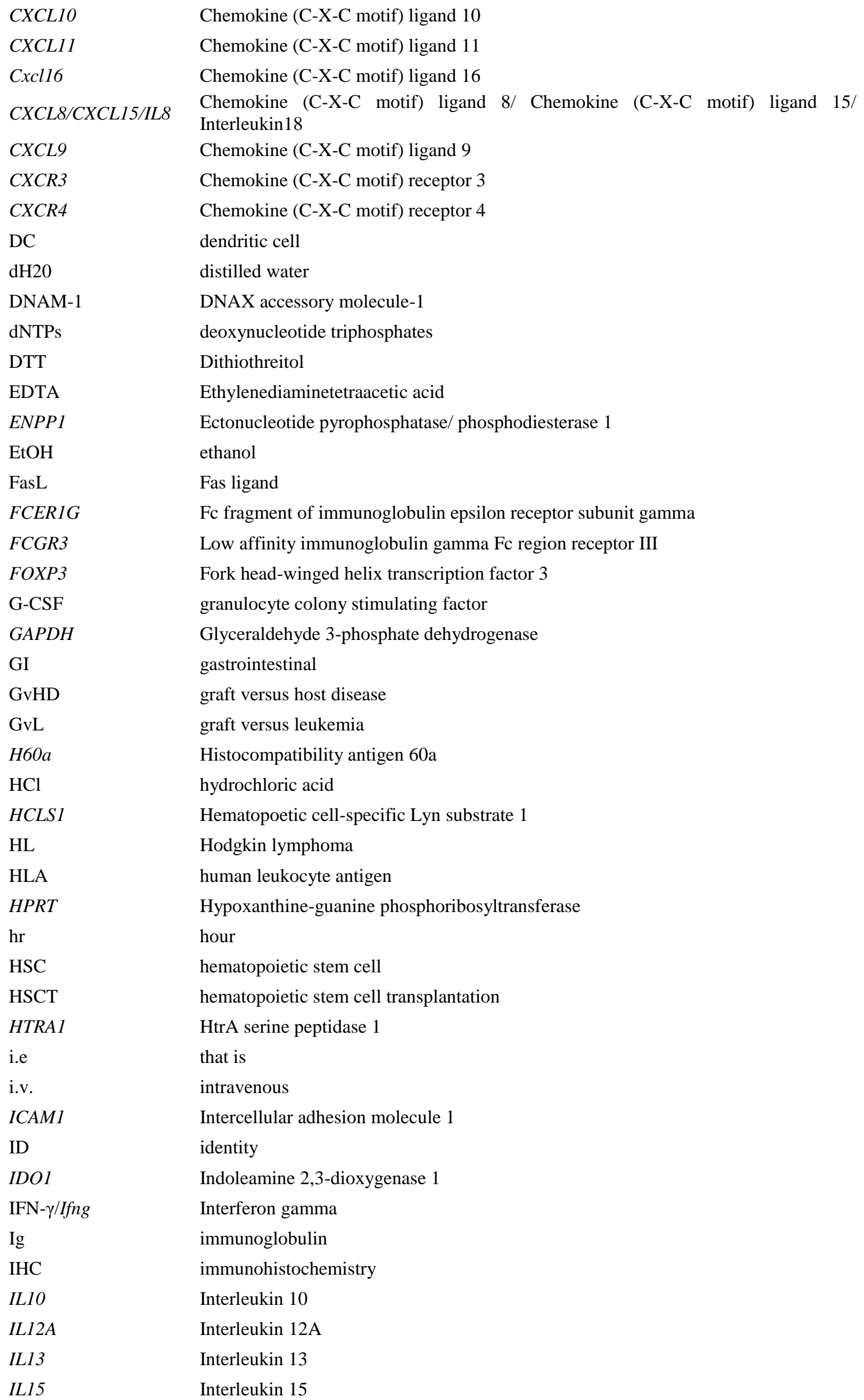

Interleukin 15 
ILI7A

ILIRI

ILIRL2

IL2

IL22

IL23A

IL2Ra

IL33

ILA

ILARa

IL5

IL6

iTreg

KIR

KLRK1

$\mathrm{L}$

1

LGALS3

LGALS7

LILRA5

LPS

LST1

$\mathrm{M}$

M-MLV

MDS

MDSCs

mHag

MHC

MICA

$M I C B$

$\min$

mRNA

MSC

MSRI

n

$\mathrm{NaOAc}$

$\mathrm{NaOH}$

NHL

NK

NKG2D

NO

nTreg

PAMP

PBMC

PBSC

PC
Interleukin 17A

Interleukin 1 receptor 1

Interleukin 1 receptor ligand 2

Interleukin 2

Interleukin 22

Interleukin 23A

Interleukin 2 receptor alpha

Interleukin 33

Interleukin 4

Interleukin 4 receptor alpha

Interleukin 5

Interleukin 6

induced Treg

killer immunoglobulin-like receptor

Killer cell lectin-like receptor subfamily K, Member 1

ligand

liter

Lectin galactoside-binding soluble 3

Lectin galactoside-binding soluble 7

Leukocyte immunoglobulin-like receptor subfamily A member 5

lipopolysaccharide

Leukocyte-specific transcript 1

molar

Moloney murine leukemia virus

Myelodysplastic syndrome

myeloid-derived stem cells

minor histocompatibility antigen

major histocompatibility antigen

MHC Class I polypeptide-related sequence A

MHC Class I polypeptide-related sequence B

minute

messenger RNA

Mesenchymal stem cell

Macrophage scavenger receptor 1

number

Sodium acetate

Sodium hydroxide

Non-Hodgkin's lymphoma

Natural killer

NK Cell Group 2D

nitric oxide

natural Treg

pathogen-associated molecular PBMC

peripheral blood mononuclear cell

peripheral blood stem cell

Plasmocytoma 


\begin{tabular}{|c|c|}
\hline PCR & polymerase chain reaction \\
\hline$P I 3 K A P 1$ & Phosphoinositide 3 -linase adapter protein 1 \\
\hline pmol & picomolar \\
\hline PSTPIP1 & Proline-serine-threonine phosphatase-interacting protein 1 \\
\hline PTGER2 & Prostaglandin E2 receptor \\
\hline PTPN7 & Protein tyrosine phosphatase non-receptor type 7 \\
\hline PVR/Necl5 & Poliovirus receptor/ Nectin-like protein 5 \\
\hline$P V R L 2$ & Poliovirus receptor-related 2 \\
\hline qRT-PCR & quantitative real-time PCR \\
\hline $\mathrm{R}$ & Correlation coefficient \\
\hline Rael & Retinoic acid early-inducible protein 1 \\
\hline Raell & Retinoic acid early-inducible protein ligand 1 \\
\hline RefSeq & reference sequence \\
\hline RNA & Ribonucleic acid \\
\hline rpm & rounds per minute \\
\hline Rrlt & Rae1 like transcript \\
\hline RT & reverse transcriptase \\
\hline RT & room temperature \\
\hline $\mathrm{s}$ & second \\
\hline SCT & stem cell transplantation \\
\hline SNP & single nucleotide polymorphism \\
\hline TAP1 & Transporter associated with antigen processing 1 \\
\hline TCR & $\mathrm{T}$ cell receptor \\
\hline$T G F B 1$ & Transforming growth factor beta- 1 \\
\hline$T G F B 2$ & Transforming growth factor beta- 2 \\
\hline TGFBRI & Transforming growth factor beta- 1 receptor \\
\hline$T G M 2$ & Transglutaminase 2 \\
\hline Th & $\mathrm{T}$ helper cell \\
\hline TLR & toll-like receptor \\
\hline$T N F$ & Tumor necrosis factor \\
\hline TRAIL & TNF-related apoptosis inducing ligand \\
\hline Treg & regulatory $\mathrm{T}$ cell \\
\hline TREM2 & Triggering receptor expressed on myeloid cells 2 \\
\hline TRM & Transplant related mortality \\
\hline UBC & Ubiquitin $\mathrm{C}$ \\
\hline$U B D$ & Ubiquitin D \\
\hline$U L B P 1$ & UL16 binding protein 1 \\
\hline$U L B P 2$ & UL16 binding protein 2 \\
\hline$U L B P 3$ & UL16 binding protein 3 \\
\hline UIO & University of Oslo \\
\hline URD & unrelated donor \\
\hline VCAM1 & Vascular adhesion molecule 1 \\
\hline
\end{tabular}




\section{Introduction}

\subsection{Allogeneic hematopoietic stem cell transplantation}

Allogeneic hematopoietic stem cell transplantation (HSCT) is a widely used and effective treatment for several hematological malignancies as well as other acquired or inherited fatal disorders affecting the hematopoietic system (Shlomchik, 2007). Thomas and colleagues, carried out the first human allo-HSCT in 1959 (Thomas et al., 1959). Over the last decade, the number of patients receiving HSCTs has increased dramatically, currently at around 55000-60000 transplant every year (Pasquini MC, Wang Z, 2007). However, the five year survival rate post transplant is still only at $50 \%$ due to several complications, such as acute graft-versus-host disease (aGvHD) (Appelbaum, 2001; Gooley et al., 2010). Prior to the transplantation procedure, patients are typically subjected to conditioning regimen of chemotherapy or radiotherapy and suppression of the immune system. This allows for engraftment in the recipient, thus reducing the risk of graft rejection and lowers the number of malignant cells. After transplantation, the donor hematopoietic stem cells replace the immune system of the host gradually over time (Deeg and Storb, 1985). However, patients undergoing allo-HSCT are prone to develop serious complications such as relapse of disease, GvHD, graft rejection and a variety of infections that can be fatal (Welniak et al., 2007). Several factors can affect the extent of the complications post transplant, such as patient gender, age, type of disease, health status of patient, relationship to donor, etc (Gratwohl, 2012).

\subsubsection{Conditioning therapy prior to HSCT}

Prior to HSCT, conditioning regimens eradicate host stem cells to make room for the recipient graft to proliferate and differentiate. Also, it is crucial that the recipients are immunocompromised before receiving the graft to prevent graft rejection. The suppressed host immune system allows the donor stem cells from the graft to home in the bone marrow (BM) microenvironment without the risk of graft rejection. Finally, the conditioning regimen is beneficial for tackling the underlying disease and providing a long term control of the disease or in any case, reducing the leukemic cells enough to achieve graft versus leukemia $(\mathrm{GvL})$ effects. This is mainly important for patients with hematological malignancies. Conditioning regimens are generally classified as myeloablative conditioning and non-myeloablative/reduced-intensity conditioning (Bacigalupo et al., 2009). 


\subsubsection{Source of HSCs}

Initially, the source of most hematopoietic stem cells (HSCs) was the BM. BM cells are generally obtained from the anterior and posterior iliac crest of the donor while they are under spinal or general anaesthesia (Thomas et al., 1975). However, BM is no longer the only source of stem cells. Peripheral blood stem cells (PBSCs) as well as placental blood obtained from the umbilical cord after birth, have increasingly become the stem cell sources for HSCT (Bensinger et al., 1996; Gluckman et al., 1999; Ringdén et al., 2000).

Granulocyte colony stimulating factor (G-CSF) mobilized peripheral blood has been shown in a large randomized trial, to lead to a more rapid engraftment post-transplant than the use of marrow, without increasing the incidence of aGvHD (Bensinger et al., 2001; Hägglund et al., 1998). The use of PBSCs has been associated with a trend towards higher chronic GvHD (cGvHD), especially in cases of high cell numbers used (Ringdén et al., 2002; Storek et al., 1997). However, in patients with matched unrelated donors, PBSCs can be used safely without any increase in aGvHD incidence (Remberger et al., 2001). Cord blood on the other hand, is associated with a lower rate of engraftment and an increased risk of graft failure (Kurtzberg et al., 1996; Wagner et al., 1996), but a lower risk of aGvHD and cGvHD. Unfortunately, cord blood involves a relatively low cell count, thereby limiting its use mainly to children and small adults.

\subsection{Classification of GvHD}

GvHD is still the most critical risk factor of any allo-HSCT. The incidence of GvHD in patents post-transplant is still high. GvHD is classified into acute and chronic GvHD.

\subsubsection{Acute graft-versus-host disease}

Acute GvHD (aGvHD) is a severe reaction, typically occurring within 100 days post-transplant when alloreactive donor $\mathrm{T}$ cells recognize a genetic disparity compared to the host and mount an immune response against various host tissues, mainly the skin, gastrointestinal (GI) tract, liver and lungs (Ferrara et al., 2009). It is characterized as a T helper (Th)-1 type cellular response (Mohty et al., 2005). The severity of aGvHD as well as the incidence of graft failure is directly associated with the level of mismatch between human leukocyte antigen (HLA) genes between the donor and recipient (Atkinson et al., 1990; Loiseau et al., 2007; Petersdorf, 2007). Although even in cases of HLA identical donor-recipient pairs, approximately $50 \%$ of the recipients still require treatment for aGvHD due to mismatches in the minor histocompatibility antigens (mHags) (Ferrara et al., 2009). The mHags are polymorphic peptides, encoded mostly outside the MHC locus, that are 
formed due to SNPs or gene deletions. These mHags are highly immunogenic peptides that can bind to either HLA class I or class II molecules and can subsequently be presented to T cells (Dzierzak-Mietla et al. 2012). Disparity of mHags between the donor and recipient further increases the incidence of GvHD in HLA-matched, unrelated patient-donor pairs (Welniak et al. 2007).

\subsubsection{Chronic graft-versus-host disease}

Chronic GvHD (cGvHD), which typically occurs after 100 days post-transplant. The mechanisms contributing to cGvHD are not well understood, and involve a diverse range of symptoms (Shlomchik, 2007). Chronic GvHD can either be progressive, occurring in patients who already have acute GvHD, or quiescent, occurring in patients that had aGvHD previously, or finally de novo, occurring in recipients that did not develop aGvHD (Ferrara et al., 2009). It is characterized by the polarization of $\mathrm{CD}^{+} \mathrm{T}$ cells towards a Th2 type response (Skert et al., 2009) and by the elevation of B cell activating factor (BAFF) (Sarantopoulos et al., 2009).

Even though aGvHD and cGvHD are generally considered as different conditions, evidence indicates the presence of a close relationship between the risk factors for both types of GvHD (Atkinson et al., 1990). Acute GvHD frequently occurs after day 100 as late acute GvHD, occurring after the cessation of immunosuppression or after donor lymphocyte infusion. Alternatively, GvHD can present with symptoms of both acute and chronic GvHD, known as overlap syndrome (Filipovich et al., 2005). Moreover, the pathophysiology of GvHD is further complicated due to involvement of regulatory $\mathrm{T}$ cells (Treg), antigen presenting cells (APCs), regulatory B cells (Bregs) (Shimabukuro-Vornhagen et al., 2009) and mesenchymal stem cells (MSCs). In our study, we will focus on different models of aGvHD.

\subsubsection{Pathophysiology of aGvHD}

Several years ago, Billingham postulated three requirements for GvHD development, namely a graft containing immunologically competent cells, expression of tissue antigens by the recipient that are not present in the donor and inability of the recipient to mount an effective response to eliminate the transplanted cells (Billingham, 1996). This model was modified later by adding another requirement i.e., the ability of effector cells to migrate to the target tissues of aGvHD (Sackstein, 1995). 
The pathophysiology of aGvHD is described as a three phase process. Phase I involves the effects of conditioning, followed by donor $\mathrm{T}$ cell activation during phase II and finally a cellular and inflammatory effector phase III (Jaksch and Mattsson, 2005).

\section{Phase I}

Phase I mainly involves activation of APCs. Conditioning regimens, prior to HSCT, lead to tissue injury in GvHD target tissues, mainly the liver and intestinal mucosa. The tissue damage induces expression of proinflammatory cytokines, chemokines and adhesion molecules (Ferrara et al., 2009), which in turn can activate APCs (Matzinger, 2002). The main cytokines involved in this phase are TNF- $\alpha$, IL-1 and IL-6, released by damaged host tissue, described as the "cytokine storm" (Hill and Ferrara, 2000; Hill et al., 1997). The cytokine storm is responsible for activating host APCs, and subsequently activate the donor T cells (Matzinger, 2002; Shlomchik et al., 1999). Damage to the intestinal mucosa is of particular importance as it allows the translocation of lipopolysaccharides (LPS) into the intestine further enhances the activation of host APCs and exacerbates production of TNF- $\alpha$ and IL-1 by macrophages (Nestel et al., 1992). This process is associated with increase in GvHD severity (Hill and Ferrara, 2000; Hill et al., 1999). In addition to activating APCs, these cytokines can also promote antigen presentation by non-professional APCs in the host tissue and cause direct inflammation of the tissue allowing $\mathrm{T}$ cells to access the target tissues (Hill, 2009). Non-professional APCs can directly activate cytotoxic T cells and these cause tissue damage.

\section{Phase II}

In this phase, donor $\mathrm{T}$ cells are activated after the transplantation procedure. Donor $\mathrm{T}$ cells proliferate and secrete cytokines including IL-2 and IFN- $\gamma$, that increases antigen presentation and T cell recruitment, events that are crucial to aGvHD pathophysiology (Jaksch and Mattsson, 2005). Naïve donor $\mathrm{CD}^{+} \mathrm{T}$ cells are primed by dendritic cells (DCs) initiating GvHD (Matzinger, 2002). The intensity of the $\mathrm{T}$ cell response is dependent on the MHC and minor histocompatibility disparity between donor and recipient. After HSCT, both donor and host derived APCs are present in the secondary lymphoid organs (Korngold and Sprent, 1980). The donor T cells can recognize alloantigens presented by both host APCs (direct presentation) and donor APCs (indirect presentation) (Lechler et al., 2001; Shlomchik et al., 2003). 


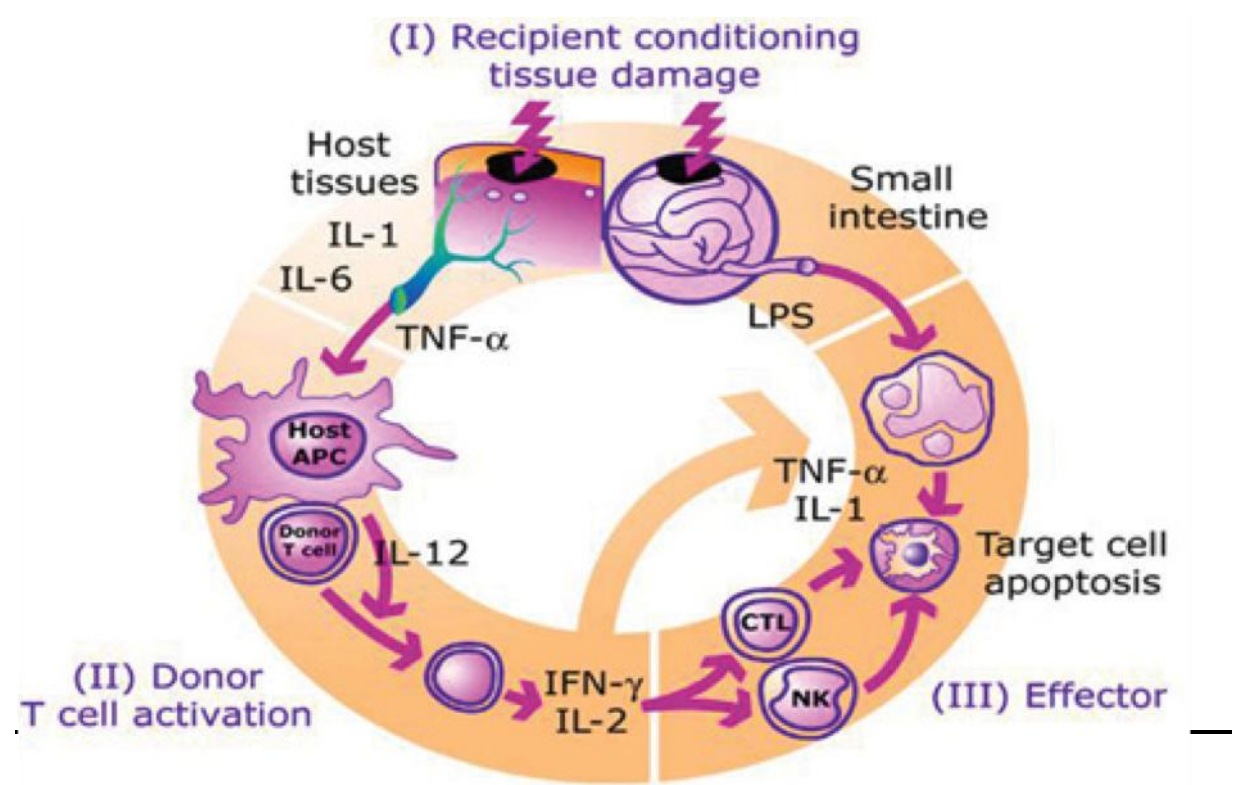

Figure 1.1 Pathophysiology of aGvHD; adopted from (Reddy, P. and Ferrara J.L.M).

Prior to transplant, the conditioning causes tissue damage, which activates APCs and increases APC function. Damage to the gut, releases bacteria, which leads to an activation of innate immune cells by PAMPs and chemokines, leading to direct damage to tissue and initiation of the cytokine storm. The cytokines further promote antigen presentation and the recruitment of effector $\mathrm{T}$ cells and innate immune cells, further augmenting the pro-inflammatory cytokine response. Finally, effector T cells, NK cells, macrophages and pro-inflammatory cytokines such as TNF- $\alpha$, IL-1 result in damage to target tissues such as skin, gut, liver and lung, leading to multi-organ failure and aGvHD.

\section{Phase III}

Phase III otherwise known as the efferent phase, involves a complex cascade of effector processes that cause further tissue injury in the host. The important mediators in this phase are the cytokines TNF- $\alpha$ and IL-1 and macrophage derived nitric oxide (NO). In addition cytotoxic T lymphocytes (CTLs) and Fas- and perforin-mediated mechanisms and NK cells are involved in the complex cascade leading to development of GvHD (Ferrara 2003). TNF- $\alpha$ and IL-1 produced by APCs further activate DCs, increasing allo-antigen presentation, further induce cytokines and recruitment of more effector cells that migrate to target organs of GvHD (Jaksch and Mattsson, 2005), causing an exacerbated tissue damage by inducing apoptosis in the target organs, such as gut, liver, lung and skin and resulting in multi-organ failure (Ferrara 1992). The mechanism of tissue injury by infiltrating alloreactive $\mathrm{T}$ cells is shown in Figure 1.2. Several chemokines and their receptors specifically guide $\mathrm{T}$ cells during this process (Wysocki et al. 2005). 


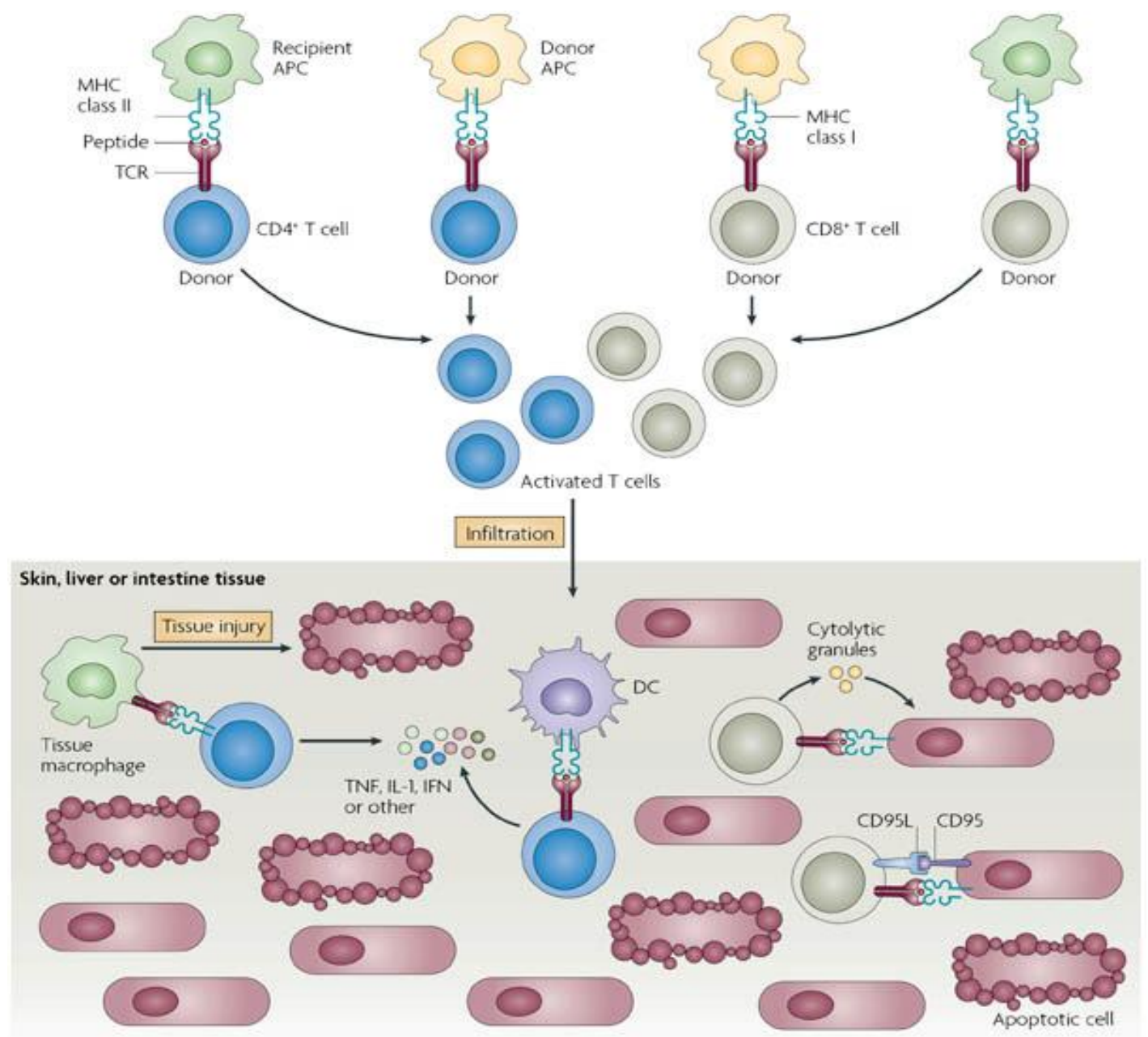

Figure 1.2: Tissue injury by infiltrating alloreactive T cells; adopted from (Shlomchik et al. 2007). Mechanisms of tissue injury in the target aGvHD tissues by infiltrating alloreactive $\mathrm{T}$ cells. Activated alloreactive $\mathrm{CD} 8^{+} \mathrm{T}$ cells directly induce tissue injury via expression of CD95 ligand and producing cytolytic granules. By contrast, $\mathrm{CD}^{+} \mathrm{T}$ cells can be activated by tissue macrophages and DCs, and subsequently release TNF- $\alpha$, IL-1 and IFN- $\gamma$. Alternatively, they can activate antigen bearing macrophages that can directly induce tissue injury.

The main pathways involved in the tissue damage to the aGvHD target tissues are: the FAS/FASL pathway, the perforin/granzyme pathway and direct tissue injury mediated by cytokines. Knockout mouse models have elucidated the importance of each of the pathways (Jaksch and Mattsson, 2005).

In the perforin/granzyme pathway, following direct cell contact, perforin penetrates the cell membrane, in turn leading to the activation of the caspase cascade and cytolysis of the cell (Goker et al., 2001). The FAS receptor (CD95) is expressed on many tissues and its expression levels are further induced in the presence of inflammation. On the other hand, the FAS receptor ligand (FASL/CD95L) is predominantly expressed on activated T cells, macrophages and neutrophils. The interaction between FAS and the FASL causes FAS-mediated apoptosis (Nagata and Golstein, 1995). During aGvHD, the expression of FASL is increased on both $\mathrm{CD}^{+}$and $\mathrm{CD} 4^{+} \mathrm{T}$ cells and 
the serum levels of both FAS and FASL correlate with a severe grade of GvHD (Jaksch and Mattsson, 2005). The FAS/FASL mechanism is mostly important in hepatic GvHD, since it has been shown that FAS-deficient recipient mice were protected from hepatic GvHD (van Den Brink et al., 2000).

\subsubsection{Histopathological manifestation of GvHD in target organs}

In 1956, Barnes and colleagues showed that when irradiated mice were infused with allogeneic bone marrow and splenic cells, they could recover from the subsequent aplasia and irradiation injury. However, the mice developed skin changes, liver abnormalities, weight loss and diarrhoea, subsequently dying from a "secondary disease" called the "runt disease" (Barnes et al., 1956), which later came to be known as GvHD. This disease mainly manifests clinically in the skin, liver and GI tract.

The first clinical symptoms of aGvHD usually present as a skin rash, which could be pruritic, occurring on the palms and soles, neck or shoulders (Ferrara et al., 2009). As the disease progresses, the rash can spread to other parts of the body and can form bullous lesions during severe aGvHD (Ferrara et al., 2009). However during cGvHD, the skin appears scleradermous and lesions are characterized by immunoglobulin deposits (Griffith et al., 2008).

The second most commonly involved organ during aGvHD is the liver. Damage to the liver typically leads to an increase in conjugated bilirubin and alkaline phosphatase, eventually leading to cholestasis. In addition, other histopathological manifestations in the liver include infiltration of lymphocytes into the bile ducts and degeneration of the biliary and epithelial cells (Snover et al., 1984).

Another organ that is affected by GvHD is the GI tract, which is clinically characterized by diarrhoea, abdominal cramp, nausea and vomiting (Ferrara et al., 2009). The GI tract is also most affected after conditioning. These symptoms can be accompanied by blood and mucosa in stool, as well as weight loss. The histopathology of the damage to the GI tract presents with necrosis of individual cells, loss of individual crypts, lymphocyte infiltration in the lamina propria and mucosal epithelium erosion (Ferrara et al., 2009; Snover et al., 1985). 


\subsection{Important HLA and non-HLA related risk factors involved in aGvHD}

\subsubsection{Human leukocyte antigens}

The Major Histocompatibility Complex (MHC) encoding Human Leukocyte Antigens (HLAs) is located on the chromosome 6 in humans (Beck and Trowsdale, 2000). HLA genes are classified into three classes, namely HLA class I, HLA class II and HLA class III. The class I region is comprised of the classical HLA-A, HLA-B and HLA-C genes, that are present on all nucleated cells, whereas the class II region contains the HLA DR, DQ and DP genes that are expressed by professional APCs (Beck and Trowsdale, 2000). Under certain conditions such as inflammation and cell damage, the HLA class II proteins can be expressed by other cell types as well. The manifestation of aGvHD is predominantly a result of mismatch between donor and recipient HLA proteins (Lee et al., 2007). Even siblings only have a $25 \%$ possibility of being matched for the MHC. However, even when siblings are matched for the MHC, around $40 \%$ of patients that undergo HSCT still develop GvHD (Ferrara et al., 2009), as a consequence of mismatched in the mHags. Differences in the mHags between MHC-matched donor and recipients leads to the risk of GvHD in matched donors (Welniak et al., 2007).

\subsubsection{Killer immunoglobulin receptors}

Killer immunoglobulin receptors (KIRs) are present on NK cells, and subpopulations of $\gamma \delta$ and $\alpha \beta$ T cells (Uhrberg et al., 2001) and are inherited independent of the HLA (Welniak et al., 2007). KIRs are comprised of both activatory and inhibitory receptors responsible for regulating NK cell activation. They can recognize HLA-A, B and C molecules. The relevance of KIRs for HSCT outcome has been emphasized by several studies. Lack of engagement of inhibitory NK cell receptors by recipient ligands, was associated with beneficial NK cell alloreactivity (Ruggeri et al., 2002). KIR/KIR ligand incompatibilities have been associated with effects in graft rejection, GvHD and GvL effects (Ruggeri et al., 2002).

\subsubsection{Co-stimulatory molecules}

The stimulation of $\mathrm{T}$ cells involves the recognition of peptides presented on APCs as well as a second co-stimulatory signal. The expression of co-stimulatory molecules is important for regulating the differentiation, proliferation and activation of $\mathrm{T}$ cells. Both activating CD28 and inhibitory cytotoxic T lymphocyte antigen 4 (CTLA4) costimulatory molecules are present on T 
cells, while their ligands CD80 (B7-1) and CD86 (B7-2) are expressed primarily on APCs (Manickasingham et al., 1998). Binding of costimulatory molecules to their ligands promotes differentiation and survival of T cells stimulation (Alegre et al., 2001). Other pathways involved in T cell activation, NKG2D-NKG2D-L (Karimi et al. 2015), DNAM-1/DNAM-1-L (Nabekura et al., 2010), and the ICOS/ICOS-L (Taylor et al., 2005) have also been implicated in aGvHD severity.

\subsection{Effector cells involved in GvHD}

\subsubsection{T cells}

Mature $T$ cells present in the bone marrow are crucial in inducing GvHD, since elimination of $T$ cells from the graft prevents GvHD (Trentin and Judd, 1973; Tyan, 1973). The exact T cell subsets that are involved in GvHD induction largely depend on the histocompatibility disparity between the donor and recipient. Donors and recipients that are fully MHC-mismatched elicit a severe GvHD reaction by both $\mathrm{CD}^{+}$and $\mathrm{CD} 8^{+}$donor T cells (Müller-Ruchholtz et al., 1976), while an MHC-II mismatched model requires only donor $\mathrm{CD}^{+} \mathrm{T}$ cells to induce a GvHD response (Korngold and Sprent, 1985). Similarly, when the donor and recipient are MHC-I mismatched, donor $\mathrm{CD}^{+} \mathrm{T}$ cells alone, without any participation from donor $\mathrm{CD}^{+} \mathrm{T}$ cells are enough to induce a GvHD response (Sprent et al., 1986, 1988; Theiss-Suennemann et al., 2015). However, the severity of the response is heightened with the presence of $\mathrm{CD} 4^{+} \mathrm{T}$ cells in addition to the $\mathrm{CD} 8^{+} \mathrm{T}$ cells in the graft, despite no mismatches in the MHC-II molecules (Korngold and Sprent, 1982; Sprent et al., 1988). In addition, $\mathrm{CD}^{+} \mathrm{T}$ cells further exacerbate the alloreactivity of $\mathrm{CD}^{+} \mathrm{T}$ cells during GvHD, due to infections by endogenous viruses such as herpes simplex, cytomegalovirus (CMV) and varicella zoster virus (Cray \& Levy 1990; Cray \& Levy 1990; Ringdén 1992), causing a more intense GvHD reaction (Cray and Levy, 1993). Across mismatches in mHags, GvHD is mostly induced by donor CD8 ${ }^{+} \mathrm{T}$ cells (Korngold and Sprent, 1982), although $\mathrm{CD}^{+} \mathrm{T}$ cells are mainly involved in production of immune-regulatory cytokines (OKunewick et al., 1987). Cytokines released drive the differentiation of donor $\mathrm{CD}^{+} \mathrm{T}$ cells into distinct Th subsets. DCs or NK cells secrete IFN- $\gamma$, IL-12, and IL-18 cytokines that drive Th1 differentiation, which in turn leads to the production of IL-2 and IFN- $\gamma$ (Kurt-Jones et al., 1987). Th2 cells are induced by IL-4 produced by basophils and mast cells, and secrete IL-4, IL-5, IL-10 and IL-13 (Heinzel et al. 1991). Moreover, while Th1 cells respond to chemokines up-regulated in response to Th1 cytokines via their expression of chemokine receptors CCR-5 and CXCR-3 (Moser et al., 2004; Sallusto et al., 1998), Th2 cells express the skin-homing chemokine receptor CCR-4 together with the CCR-3 receptor (Campbell et al., 1999; Sallusto et al., 1998), which may explain their association to cutaneous GvHD. A third T helper subset, Th17 cells 
are induced by either IL-23 or a combination of IL- 6 and TGF- $\beta$, and produce IL-17, IL-21 and IL22 (Harrington et al., 2005).

\subsubsection{Regulatory $\mathrm{T}$ cells}

Another important subset of $\mathrm{T}$ cells, the regulatory $\mathrm{T}$ cells (Tregs) have been the focus of several HSCT studies due to their ability to suppress alloreactivity (Fontenot et al., 2005). Tregs, defined as $\mathrm{CD} 4{ }^{+} \mathrm{CD} 25^{+}$forkheadbox protein $3(\mathrm{FOXP} 3)^{+} \mathrm{T}$ cells, are involved in the maintenance of immunological tolerance by secreting anti-inflammatory cytokines such as TGF- $\beta$ and IL-10 (Beres and Drobyski, 2013). Tregs can be divided into two populations, thymus-derived naturally occurring Tregs (nTregs) (Sakaguchi et al., 1995) and adaptive or induced Tregs (iTregs) generated in the peripheral lymphoid organs in the presence of TGF- $\beta$ (Cobbold et al., 2004). However its exact role during GvHD is not clear. Previously studies showed that the frequency of $\mathrm{CD} 4{ }^{+} \mathrm{CD} 25^{+}$ Tregs was significantly lower in patients with severe acute or chronic GvHD, and a decreased level of $\mathrm{CD}^{+} \mathrm{CD} 25^{+}$Tregs was correlated to increased severity of GvHD (Li et al., 2010). In contrast, Foxp $3^{+}$cells were upregulated significantly in GvHD intestinal mucosa when compared to non GvHD mucosa in another study (Lord et al., 2011).

\subsubsection{NK Cells}

NK cells are crucial for successful engraftment after HSCT. As in T cells, NK cells can also migrate to and proliferate in lymphoid organs, and can also reach target tissues during aGvHD. In HSCT, NK cells reduce GvHD by producing TGF- $\beta$ or stimulate its production in other cells (Ruggeri et al., 2002). NK cells are also known to inhibit activated alloreactive T cells, and thereby reduce GvHD while maintaining GvL effects (Olson et al., 2010). On the other hand, they also produce IFN- $\gamma, \mathrm{TNF}-\alpha$ and NO upon induction, resulting in subsequent tissue injury during aGvHD (Cooke et al., 1998). 


\subsubsection{B cells}

Antigen presentation by $\mathrm{B}$ cells also plays an important role during immune responses. B lymphocytes become potent antigen-presenting cells (APCs) on activation via the B-cell receptor (BCR) and co-stimulatory receptors such as CD40 (Von Bergwelt-Baildon et al., 2002). Furthermore, the activated $\mathrm{B}$ cells can prime both $\mathrm{CD} 4^{+}$and $\mathrm{CD} 8^{+} \mathrm{T}$ cells, and the $\mathrm{T}$ cell response is dependent on antigen presentation by B cells (Von Bergwelt-Baildon et al., 2002; Constant et al., 1995). Regulatory B lymphocytes can also induce the secretion of several cytokines such as TGF- $\beta$ and chemokines, and reduce T cell responses (Mauri and Ehrenstein, 2008). B cells have mainly been associated with cGvHD. Elevated mRNA expression levels of the B cell-activating factor $(B A F F)$ is associated with clinical cGvHD in patient biopsies (Ahmed et al., 2015; Allen et al., 2012). Moreover, the depletion of B cells reduced the incidence of cGvHD in mice (Schultz et al., 1995).

\subsubsection{Other inflammatory effectors affecting aGvHD}

\subsubsection{Nitric Oxide}

Nitric oxide (NO) plays a crucial role in host defense and anti-microbial function of macrophages. IFN- $\gamma$ also induces the production of NO. Exposure to increased amounts of IFN- $\gamma$ reduces the amount of LPS needed to trigger synthesis of inflammatory mediators by macrophages (Ding et al., 1988; Gifford and Lohmann-Matthes, 1987). As a result, even small quantities of LPS can trigger NO and TNF- $\alpha$ production (Kichian et al., 1996; Nestel et al., 1992).

Elevated serum levels of NO precede GvHD symptoms in both human and animal aGvHD (Langrehr et al., 1992; Weiss et al., 1995). NO induces immunosuppression and inhibiting mechanisms that repair the target tissues, by inactivating non-heme-iron containing enzymes, in turn inhibiting epithelial stem cell proliferation in the skin and gut (Krenger et al., 1996; Nestel et al., 1992), and causing direct tissue damage (Halliwell and Gutteridge, 1992).

\subsubsection{Cytokines and chemokines}

During the pathophysiology of aGvHD, in addition to LPS, several proinflammatory cytokines such as IL-1, TNF- $\alpha$ or IFN- $\gamma$, are involved in stimulating different cells such as macrophages, epithelial, endothelial, fibroblast cells, resident cells and monocytes (Jaksch and Mattsson, 2005). 
TNF- $\alpha$ is involved in activating DCs and enhancing the presentation of alloantigens. As a result of stimulation, inflammatory chemokines are expressed in the infiltrating cells, and the chemokines recruit effector $\mathrm{T}$ cells, monocytes and granulocytes to the sites of inflammation. On reaching the target organs, $\mathrm{T}$ cells cause damage to target tissues via cytotoxic activity directly, leading to recruitment of other leukocytes. The cytotoxic activity of the T cells is mediated mainly by the Fas ligand/Fas and the perforin-granzyme pathways (Braun et al., 1996). However, CTLs deficient for both pathways exhibit residual cytolytic activity, suggesting that other pathways, like the TNFrelated apoptosis inducing ligand (TRAIL) might be involved in mediating GvL but not GvHD (Schmaltz et al., 2002). TNF- $\alpha$ can be expressed and secreted by activated CTLs, contributes to the cytotoxicity mediated by CTLs (Ware et al., 1995). The TNF- $\alpha$ can be derived from monocytes and macrophages of either the donor or the host (Ferrara et al., 1999). Moreover, TNF- $\alpha$ derived from donor $\mathrm{T}$ cells have been associated with morbidity and mortality due to aGvHD as well as GvL (Schmaltz et al., 2003). TNF- $\alpha$ is involved in both, the induction and the effector phases of GvHD (Ferrara and Deeg, 1991). Furthermore, activated macrophages can secrete inflammatory cytokines that can strongly contribute to the tissue damage during the last phase of aGvHD. Interestingly, TNF- $\alpha$ has also been associated with direct tissue damage by inducing necrosis of target cells and apoptosis (Wall and Sheehan, 1994).

IL-1 is another important cytokine that plays an important role in the effector phase of acute GvHD. Mice receiving IL-1 after allo-SCT, had an increased frequency of mortality, which was augmented in aGvHD (Atkinson et al., 1991). Moreover, IL1 mRNA was significantly increased in mononuclear cells during clinical aGvHD (Tanaka et al., 1995a). Administration of an IL-1 receptor antagonist (IL-1Ra) was shown to attenuate aGvHD in mice (Eisenberg et al., 1990; Hannum et al., 1990), however the treatment was not successful in preventing aGvHD in a randomized clinical trial (Antin et al., 2002).

Several cytokines and chemokines have been implicated during aGvHD. Moreover, several therapy strategies for aGvHD focus on blocking interactions between chemokines and their receptors. For example, an ameliorated gastrointestinal aGvHD in mouse models was observed on administration of anti-CXCR-3 antibodies (He et al., 2008).

\subsection{Regulation of cytokine genes in aGvHD}

Several studies have elucidated the importance of cytokine expression during HSCT. A number of cytokines are differentially expressed during GvHD, the most important being IFN- $\gamma$, IL-2 and 
TNF- $\alpha$ that are involved in the initiation of aGvHD (Reddy, 2003). Several SNPs in the cytokine genes encoding IL-10, TNF- $\alpha$ and IL- 6 have been associated with an increased risk of GvHD.

The activation of cytokines is determined by the difference in polarization of $\mathrm{T}$ cells into mainly type 1 and type 2 responses, which in turn determines the immune response during aGvHD, and IL-12 is involved in the activation of type 1 cytokines (Yang et al., 1997). Several gene expression studies have reported the involvement of several cytokines that are involved in aGvHD (Buzzeo et al., 2008; Das et al., 2001; Tanaka et al., 1995a).

IL-2 is a Th1 cytokine that serves as a T cell growth factor. The treatment and prophylaxis of aGvHD frequently involves the inhibition of IL-2 production by using cyclosporine A (Qian et al., 2013). In both animal and clinical studies, the administration of monoclonal antibodies against the IL-2 receptor after transplant prevented aGvHD (Blaise et al. 1991; Ferrara et al. 1986). IL-2 is also necessary for the generation and maintenance of Tregs, suggesting that inhibition of IL-2 could have a negative effect on the long-term tolerance after HSCT (Gavin et al., 2007; Zeiser et al., 2006).

IFN- $\gamma$ is a pro-inflammatory cytokine that is of crucial importance during aGvHD. Several cell types, such as activated T cells, NK and NKT cells, produce IFN- $\gamma$. Both IFN- $\gamma$ and IL-2 are involved in the proliferation of $\mathrm{T}$ cells, stimulation of cytotoxic T lymphocyte (CTL) and NK cell responses and production of IL-1 and TNF- $\alpha$ (Jaksch and Mattsson, 2005). A number of studies have reported a correlation between the expression of IFN- $\gamma$ and severity of aGvHD (Das et al., 2001; Dickinson et al., 1994; Tanaka et al., 1995a). IFN- $\gamma$ is induced early in the cytokine cascade of aGvHD, and augments the disease, leading to the maturation of DCs and stimulation of macrophages to produce cytokines and NO (Jaksch and Mattsson, 2005). Moreover, the mRNA expression of $I F N G$ and $I L 2$ was increased in the PBMCs of GvHD patients and the expression of IL2 mRNA correlated with the progression of GvHD (Das et al., 2001). TNF- $\alpha$ is another proinflammatory cytokine involved in the pathogenesis of aGvHD. Neutralization of TNF- $\alpha$ can reduce aGvHD symptoms (Cooke et al., 1998).

\subsection{Regulation of chemokine genes in aGvHD}

All inflammatory reactions are associated with a recruitment of leukocytes to sites of inflammation. Many genes encoding chemokines and their receptors are regulated during GvHD. The Th1 chemokine receptor, CXCR-3 is an important chemokine receptor involved in lymphocyte recruitment and is expressed on T cells. CXCL-9, CXCL-10 and CXCL-11, the ligands for CXCR3, are induced by the Th1 cytokines IFN- $\gamma$ and TNF- $\alpha$ (Groom and Luster, 2011). CXCL-9 is 
expressed by effector $\mathrm{CD} 4^{+}$Th1 cells and $\mathrm{CD} 8^{+} \mathrm{CTL}$, and has been shown to affect the migration of effector T cells to inflamed tissue during progression of GvHD (Groom and Luster, 2011). Several studies have reported the regulation of the CXCR-3 and its ligands during aGvHD (Ahmed et al. 2015; Bouazzaoui et al. 2009; Zhou et al. 2007; Ichiba et al. 2003; Sadeghi et al. 2013). Furthermore, the use of CXCR-3-transfected Tregs, as a novel therapeutic strategy, resulted in decreased severity of GvHD due to attraction of Tregs to the target tissues of GvHD (Hasegawa et al., 2008). CCR-5 mediates the recruitment of effector $\mathrm{T}$ cells, as well as regulatory $\mathrm{T}$ cells, to many different target organs (Murai et al., 1999; Wysocki et al., 2004, 2005b). Additionally, in gastrointestinal aGvHD, the chemokine receptors CXCR-3 (Duffner et al., 2004) and CCR-6 (Varona et al., 2006) and chemokine CX3CL1 (Ueha et al., 2007), have been shown to play important roles. CCL-2, CCL-3, CCL-4 and CCL-5 are involved in the migration of donor cells to the target organs during GvHD (Castor et al., 2012). The mRNA expression of the chemokines (Cxcl1,Cxcl2, Cxcl9 and Cxcl20,Ccl2, Ccl5, Ccl6, Ccl7, Ccl8,Ccl9, Ccl11, Ccl17, and Ccl29) and chemokine receptors $(C c r l$ and $C c r 5)$ were reported to be increased in the skin during aGvHD (Sugerman et al. 2004; Zhou et al. 2007). Ccl5 was also profoundly up regulated during hepatic aGvHD in a mouse model (Ichiba et al. 2003).

\subsection{MRNA expression during aGvHD}

Several studies have shown the importance of determining the regulation of genes involved in different aspects of HSCT and in aGvHD. A number of gene expression-profiling studies have identified candidate genes that could be associated with the disease diagnosis, prognosis and outcome aGvHD (Bouazzaoui et al., 2009; Novota et al., 2011; Sadeghi et al., 2013; Sugerman et al., 2004; Verner et al., 2012). Moreover, identification of the genes that are regulated during the different phases of aGvHD, have been important in determining potential therapeutic strategies. For example, blocking cytokines, such as TNF- $\alpha$ (Cooke et al., 1998) and cytokine receptors such as IL-6R (Chen et al., 2009; Tawara et al., 2011) was associated with a reduction in aGvHD. Alternatively, some cytokines are protective against aGvHD, such as IL-22 (Hanash et al., 2012). In this study, we aimed to compare the gene expression patterns in different aGvHD target organs in three different species, between aGvHD tissue and controls without aGvHD. 


\subsubsection{Expression of previously identified candidate genes in rat skin and clinical aGvHD skin biopsies}

MHC matching is essential to reduce the risk GvHD but mHags also affect the outcome of HSCT. In addition, SNPs, even if not giving rise to mHags, can influence the result of HSCT. Previously, 11 non-class I/II MHC and 174 other genes were found to be regulated during $\mathrm{GvH}$ reactions (Novota et al., 2011) in rat skin explant assays, which provide an in vitro model of GvHD (Novota et al., 2008) as well as in rat GvHD models (Zinöcker et al., 2011). The expression of 27 genes was tested in human skin explant assays and in human GvHD skin biopsies (Norden et al., unpublished data). The candidate genes selected from their study can be divided into three groups. The first group includes genes regulated by IFN- $\gamma$, TAP1, LILRA5, UBD, TREM2, PTGER2 and MSR1. In this group, the gene expressions of these genes were increased in the rat and skin biopsies, while LST1 mRNA was downregulated. The second group of candidate include genes regulated by B and T cell activation, CARD11, HCLS1, PIK3AP1, PSTPIP1 and PTPN7, which were all increased in the rat skin and human clinical aGvHD skin biopsies (Dressel et al., 2013; Novota et al., 2011). The last group consists of genes that are associated with innate immune responses. In this group, ANP32A, ClQTNF7, HTRA1, LGALS7 were downregulated, whereas TGM2 was upregulated in the human and rat aGvHD skin (Dressel et al., 2013; Novota et al., 2011).

In this study, we wanted to validate the regulation of these candidate genes in different tissues using a larger sample size, and to confirm the trend of regulation of these genes in aGvHD. Therefore, we compared their expression patterns in the different target organs of mice, and determined the effect of preconditioning on their regulation patterns. We already know these genes are differentially regulated in the human and rat skin during aGvHD. Additionally, we wanted to determine if these genes are also regulated in a different rat (MHC congenic) model in the different aGvHD tissues, and more importantly, to compare their expression patterns to the GI biopsies from out human aGvHD cohort. Identifying genes that are similarly regulated in the different target organs, or more importantly in the different species can help us to better understand the mechanisms during aGvHD. 
Table 1.1: Previously identified candidate genes from (Novota et al., 2011) and patent (Publication Number: 20130338035) (Dressel et al., 2013).

Genes are listed in alphabetical order

\begin{tabular}{|c|c|c|}
\hline Symbol & Entrez Gene Name & Location of protein \\
\hline ANP32A & $\begin{array}{l}\text { acidic (leucine-rich) nuclear phosphoprotein } 32 \\
\text { family, member A }\end{array}$ & Nucleus \\
\hline C1QTNF7 & $\mathrm{C} 1 \mathrm{q}$ and tumor necrosis factor related protein 7 & Extracellular Space \\
\hline$C A R D 11$ & caspase recruitment domain family member 11 & Cytoplasm \\
\hline CXCL9 & chemokine (C-X-C motif) ligand 9 & Extracellular Space \\
\hline HCLS1 & hematopoietic cell-specific Lyn substrate 1 & Nucleus \\
\hline HTRAI & HtrA serine peptidase 1 & Extracellular Space \\
\hline ILIRL2 & interleukin 1 receptor like 2 & Plasma Membrane \\
\hline$L G A L S 7 / L G A L S 7 B$ & lectin, galactoside-binding, soluble, 7 & Extracellular Space \\
\hline LILRA5 & leukocyte immunoglobulin like receptor A5 & Plasma Membrane \\
\hline LST1 & leukocyte specific transcript 1 & Cytoplasm \\
\hline MSR1 & macrophage scavenger receptor 1 & Plasma Membrane \\
\hline PIK3AP1 & phosphoinositide-3-kinase adaptor protein 1 & Cytoplasm \\
\hline PSTPIP1 & $\begin{array}{l}\text { proline-serine-threonine phosphatase interacting } \\
\text { protein } 1\end{array}$ & Cytoplasm \\
\hline PTGER2 & prostaglandin E receptor 2 & Plasma Membrane \\
\hline PTPN7 & protein tyrosine phosphatase, non-receptor type 7 & Cytoplasm \\
\hline TAP1 & $\begin{array}{l}\text { transporter 1, ATP-binding cassette, sub-family B } \\
\text { (MDR/TAP) }\end{array}$ & Cytoplasm \\
\hline TGM2 & transglutaminase 2 & Cytoplasm \\
\hline TREM2 & triggering receptor expressed on myeloid cells 2 & Plasma Membrane \\
\hline$U B D$ & ubiquitin D & Nucleus \\
\hline
\end{tabular}

\subsubsection{Other selected focus genes}

In addition, we also selected several cytokines, chemokines and their receptors, and genes associated with immune cells in aGvHD, listed in Table 1.2. Secretion of inflammatory cytokines and chemokine expression are important in activation of lymphocytes and their migration to sites of inflammation (Ebert et al., 2005; Hill et al., 1997; New et al., 2002). Previously, studies have shown an increased expression of recruiting chemokines in target tissues of aGvHD in mice (Ma et al., 2011; Mapara et al., 2006). Therefore, we expect these genes to be significantly regulated during aGvHD in one more of the target organs as many of the chemokines and cytokines have been implicated to be important in aGvHD pathophysiology previously. The importance of this study is to elucidate the regulation patterns of these genes in different tissues of the mouse aGvHD model, and more importantly, assess the effects of preconditioning on the expression pattern of these genes in the different target organs. Furthermore, comparing the gene expression profiles between the 
different target organs to spleen, the non-target organ, could potentially help us understand the varying mechanisms of $\mathrm{T}$ cell trafficking in the different organs in aGvHD.

Table 1.2: List of further selected focus genes.

\begin{tabular}{|c|c|c|}
\hline Symbol & Entrez Gene Name & Location of protein \\
\hline CCL4 & chemokine (C-C motif) ligand 4 & Extracellular Space \\
\hline CCL5 & chemokine (C-C motif) ligand 5 & Extracellular Space \\
\hline CCL9 & chemokine (C-C motif) ligand 9 & Extracellular Space \\
\hline CCR1 & chemokine (C-C motif) receptor 1 & Plasma Membrane \\
\hline CCR4 & chemokine (C-C motif) receptor 4 & Plasma Membrane \\
\hline CCR5 & $\begin{array}{l}\text { chemokine (C-C motif) receptor } 5 \\
\text { (gene/pseudogene) }\end{array}$ & Plasma Membrane \\
\hline CX3CL1 & chemokine (C-X3-C motif) ligand 1 & Extracellular Space \\
\hline CX3CR1 & chemokine (C-X3-C motif) receptor 1 & Plasma Membrane \\
\hline CXCL10 & chemokine (C-X-C motif) ligand 10 & Extracellular Space \\
\hline CXCL11 & chemokine (C-X-C motif) ligand 11 & Extracellular Space \\
\hline CXCL15 & chemokine (C-X-C motif) ligand 15 & Extracellular Space \\
\hline CXCL16 & chemokine (C-X-C motif) ligand 16 & Extracellular Space \\
\hline CXCR3 & chemokine (C-X-C motif) receptor 3 & Plasma Membrane \\
\hline CXCR4 & chemokine (C-X-C motif) receptor 4 & Plasma Membrane \\
\hline IFNG & interferon, gamma & Extracellular Space \\
\hline IL10 & interleukin 10 & Extracellular Space \\
\hline IL12A & interleukin $12 \mathrm{~A}$ & Extracellular Space \\
\hline IL13 & interleukin 13 & Extracellular Space \\
\hline IL15 & interleukin 15 & Extracellular Space \\
\hline IL17A & interleukin 17A & Extracellular Space \\
\hline ILIRI & interleukin 1 receptor, type I & Plasma Membrane \\
\hline$I L 2$ & interleukin 2 & Extracellular Space \\
\hline IL22 & interleukin 22 & Extracellular Space \\
\hline IL23A & interleukin 23 subunit alpha & Extracellular Space \\
\hline$I L 2 R A$ & interleukin 2 receptor subunit alpha & Plasma Membrane \\
\hline IL33 & interleukin 33 & Extracellular Space \\
\hline IL4 & interleukin 4 & Extracellular Space \\
\hline$I L 4 R$ & interleukin 4 receptor & Plasma Membrane \\
\hline IL5 & interleukin 5 & Extracellular Space \\
\hline IL6 & interleukin 6 & Extracellular Space \\
\hline TGFB1 & transforming growth factor beta 1 & Extracellular Space \\
\hline$T G F B 2$ & transforming growth factor beta 2 & Extracellular Space \\
\hline$T G F B R 1$ & transforming growth factor beta receptor I & Plasma Membrane \\
\hline$T N F$ & tumor necrosis factor & Extracellular Space \\
\hline FOXP3 & forkhead box P3 & Nucleus \\
\hline IDO1 & indoleamine 2,3-dioxygenase 1 & Cytoplasm \\
\hline
\end{tabular}




\begin{tabular}{lll} 
ARG1 & arginase 1 & Cytoplasm \\
LGALS3 & lectin, galactoside-binding, soluble, 3 & Extracellular Space \\
BMPR1A & bone morphogenetic protein receptor type IA & Plasma Membrane \\
ENPP1 & $\begin{array}{l}\text { ectonucleotide } \\
\text { pyrophosphatase/phosphodiesterase 1 }\end{array}$ & Plasma Membrane \\
FCER1G & Fc fragment of IgE receptor Ig & Plasma Membrane \\
FCGR2A & Fc fragment of IgG receptor IIa & Plasma Membrane \\
\hline ICAM1 & intercellular adhesion molecule 1 & Plasma Membrane \\
UBC & ubiquitin C & Cytoplasm \\
\hline
\end{tabular}

In addition, our research group previously studied the effect of MHC class I chain-related molecule A (MICA)-129Met/Val dimorphism on the outcome of HSCT (Isernhagen et al., 2015). Presence of the Met allele in patients decreased the risk of death and lowered the mortality due to aGvHD, despite a higher risk to experience this complication. The functional consequences of this SNP for NKG2D signaling on NK and T cells were characterized and they suggest a causative effect of this SNP on the outcome of HSCT (Isernhagen et al., 2015, 2016a). Also, we found the expression intensity of the MICA-129 polymorphisms affected NKG2D function and cytotoxicity on NK and $\mathrm{CD} 8^{+} \mathrm{T}$ cells. This could suggest that the gene expression of NKG2D and its ligands could be important for the HSCT outcome and aGvHD (Isernhagen et al., 2016b).

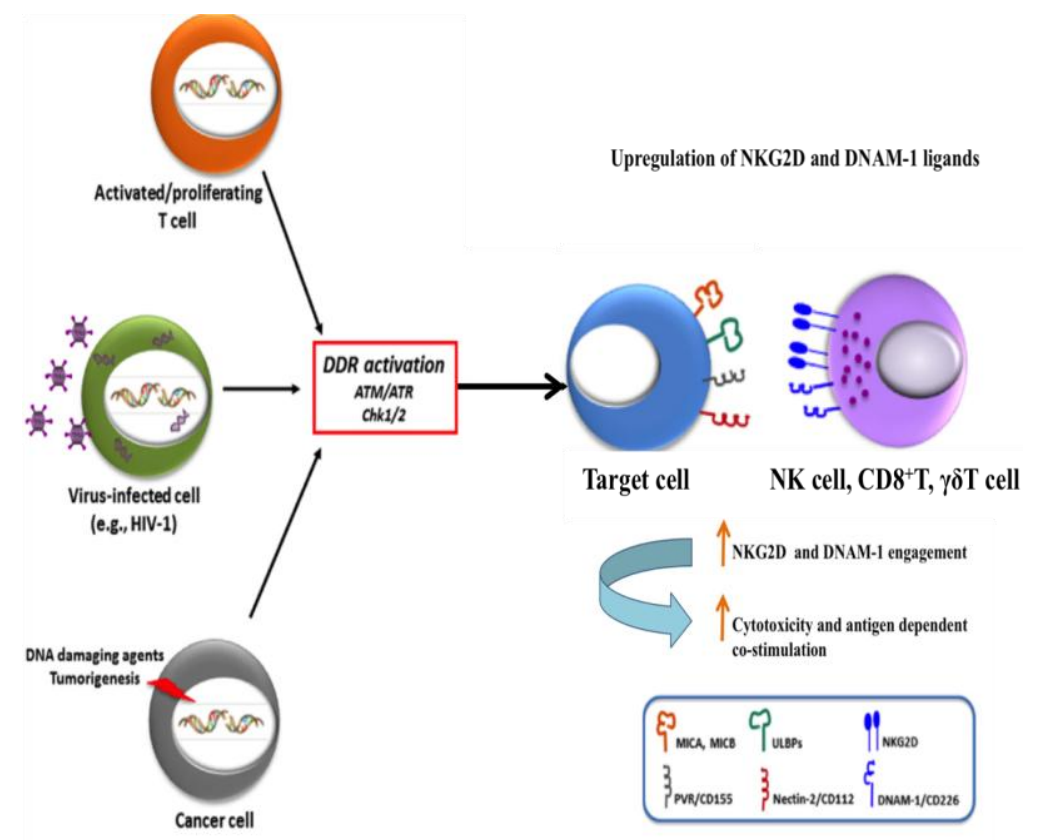

Figure 1.3: Upregulation of NKG2D and DNAM-1 adopted from (Cerboni et al., 2014)

Upregulation of NKG2D and DNAM-1 on interaction with their ligands, upon activation by DNA damage response (DDR) of stressed cells, activated by different stress inducing stimuli.

NKG2D and DNAM-1 are both activating NK receptors. In addition, they also serve as costimulatory molecules on $\mathrm{CD}^{+} \mathrm{T}$ cells. NKG2D is expressed on subsets of NK cells, NKT-cells, T cells, and CD8 ${ }^{+}$T cells (Bauer et al. 1999; Raulet 2003; Groh et al. 1996). Similarly, DNAM-1 is 
expressed on all NK cells, NKT cells, a subset of $\gamma \delta \mathrm{T}$ cells and all CD8 ${ }^{+} \mathrm{T}$ cells, in humans and on activated $\mathrm{CD} 8^{+} \mathrm{T}$ cells and a subset of $\mathrm{CD} 4^{+} \mathrm{T}$ cells in mice. Both these co-stimulatory molecules have been implicated in aGvHD. Mice deficient in either NKG2D (Karimi et al., 2015) or DNAM1 (Nabekura et al., 2010) show an attenuated aGvHD response. Their co-stimulatory function has been attributed to interactions between NKG2D/NKG2D-L and DNAM-1/DNAM-1L. NKG2D binds to several ligands in humans; MICA, MICB and ULBP1-6, also known as the RAET1 family, given their homology to mouse RAE1 proteins (Cerwenka et al., 2001). The MICA and MICB ligands are encoded in the human MHC (Bauer et al., 1999), but lack $\beta 2$ microglobulin and cannot bind to antigens. However, rodents lack MIC genes, and NKG2D binds to the RAE1 family, H60 family (a,b and c) (Takada et al., 2008), of which H60a is a minor histocompatibility antigen (Malarkannan et al., 1998) and UL16-protein like transcript 1 (MULT-1) (Carayannopoulos et al., 2002; Diefenbach et al., 2003) in mice. Similarly, in rats, NKG2D binds to two members of the RAE1 family, RAE1L and RRLT (Zhuo et al., 2010). On the other hand, the leukocyte adhesion molecule, DNAM-1 binds to CD112 encoded by PVRL2 or Nectin-2 and CD155 encoded by PVR in all three species (Bottino et al., 2003).

The engagement of NKG2D and DNAM-1, with their respective ligands, can provide costimulatory signals to $\mathrm{T}$ cells from non-professional APCs, in a non-classical MHC I restricted manner and can promote $\mathrm{CD} 8^{+} \mathrm{T}$ cell activity under specific conditions (Groh et al. 1996; Gilfillan et al., 2008). NKG2D also facilitates activation of $\mathrm{CD} 8^{+} \mathrm{T}$ cells during inflammation (Ogasawara et al., 2004), and its ligands are upregulated prior to allogeneic HSCT as a result of myeloblative conditioning (Ho et al., 2009). Karimi et al. showed that blockade of NKG2D on donor CD8 ${ }^{+} \mathrm{T}$ cells ameliorated aGvHD in mice, while maintaining GvL effects (Karimi et al., 2015). Moreover, it was recently shown that a polymorphism in its ligand, MICA-129 was important in HSCT outcome and occurrence of aGvHD (Isernhagen et al., 2015, 2016b). Interestingly, the NKG2D dependent activation of $\mathrm{NK}$ cells and the co-stimulation of $\mathrm{CD}^{+} \mathrm{T}$ cells was significantly influenced by the expression intensity of the MICA-129 variants (Isernhagen et al., 2016a). Therefore, we wanted to investigate the regulation of mRNA expression of $N K G 2 D$ and its ligands in two models of aGvHD.

The ligands are stress-induced and not expressed under normal conditions, becoming upregulated under cellular or genotoxic stress during disease or infection, such as cytomegalovirus (CMV) (Cosman et al., 2001), in turn leading to activation of NK cells and stimulation of $\mathrm{CD}^{+} \mathrm{T}^{-}$cells (Groh et al. 1996). To assess the gene expression pattern of these receptors and their ligands, we also studied the regulation the KLRK1, encoding NKG2D and CD226, encoding DNAM-1, and their ligands in different target tissues and compared their regulation in the different species in this study, listed in Table 1.3. 
Table 1.3: Genes encoding NKG2D, DNAM-1 and their ligands.

\begin{tabular}{|c|c|c|}
\hline Symbol & Entrez Gene Name & Location \\
\hline$K L R K 1$ & $\begin{array}{l}\text { killer cell lectin-like receptor subfamily } \\
1\end{array}$ & Plasma Membrane \\
\hline MICA & MHC Class I chain related protein A & Plasma Membrane \\
\hline MICB & MHC Class I chain related protein B & Plasma Membrane \\
\hline$U L B P 1$ & UL16 binding protein 1 & Plasma Membrane \\
\hline$U L B P 2$ & UL16 binding protein 2 & Plasma Membrane \\
\hline ULBP3 & UL16 binding protein 3 & Plasma Membrane \\
\hline H60a & histocompatibility $60 \mathrm{a}$ & Plasma Membrane \\
\hline Rae1 & retinoic acid early transcript 1 , alpha & Plasma Membrane \\
\hline Ulbp1 & UL16 binding protein & Plasma Membrane \\
\hline Rrlt & retinoic acid & Plasma Membrane \\
\hline Raet1 & Rat similar to retinoic acid early transcript $1 \mathrm{~L}$ & Plasma Membrane \\
\hline$C D 226$ & CD226 molecule, (DNAM-1) & Plasma Membrane \\
\hline$P V R L 2$ & $\begin{array}{l}\text { poliovirus receptor-related } 2 \text { (herpesvirus entry mediator } \\
\text { B) }\end{array}$ & Plasma Membrane \\
\hline$P V R$ & poliovirus receptor & Plasma Membrane \\
\hline
\end{tabular}




\subsection{Objectives}

The aims of this study are listed below:

a. To study the regulation of genes selected in different mouse tissues affected by aGvHD

b. To assess the difference in gene regulation due to preconditioning and aGvHD in the different mouse tissues

c. To validate the gene expression of previously identified candidate genes in the different species; mouse, rat and human GI aGvHD

d. To study the gene expression patterns of important genes associated with different immune cells during aGvHD during mouse aGvHD and compare it to human clinical gastrointestinal biopsies

e. To study the regulation of the NK receptors KLRK1 and CD226 and their ligands in the different species during aGvHD.

f. To study the regulation of genes and their effects on HSCT outcome in a patient cohort

Gene expression studies frequently use blood, whereas conjunctiva is commonly used to study cGvHD for the mRNA studies. The advantage of using animal models for studying the gene expression is the broad availability of specific target tissues of GvHD, such as liver and gut. In our study, we had a unique opportunity to study the expression patterns of several genes not only in the different target tissues during aGvHD in two different animal models of aGvHD, as well as in human biopsies. 


\section{Materials and methods}

\subsection{Materials}

\subsubsection{Enzymes}

Table 2.1: List of Enzymes.

\begin{tabular}{ll} 
Enzyme & Supplier \\
\hline \hline DNase I & NEB \\
M-MLV Reverse Transcriptase & Promega \\
RevertAid Reverse Transcriptase & Thermo Scientific \\
Proteinase K & Merck \\
RNAse A & Roche Diagnostics \\
RNAsin & NEB \\
RiboLock RNAse Inhibitor & Thermo Scientific \\
Taq DNA Polymerase & NEB \\
Exo I & NEB
\end{tabular}

\subsubsection{Antibodies}

Table 2.2: List of antibodies.

Antigen

Rat anti-mouse CD3

Biotin goat anti-rat IgG

Rat IgG1

\section{Supplier}

Bio-Rad

Biolegend

Biolegend

\subsubsection{Chemicals and Reagents}

Table 2.3: List of chemicals and reagents.

Chemical/Reagent

Acetic acid

Chloroform

\section{Supplier}

Merck 
Dithiothreitol (DTT)

\begin{tabular}{|c|c|}
\hline dNTPs & NEB \\
\hline dNTP mix & Thermo Scientific \\
\hline Eosin Y & Merck Millipore \\
\hline Ethanol (analytical grade) & UMG Pharmacy \\
\hline Ethanol (EtOH) & UMG Pharmacy \\
\hline Ethidium bromide & HyClon \\
\hline EDTA & Carl Roth \\
\hline GeneRuler 1kb DNA ladder & Thermo Scientific \\
\hline Hydrochloric acid $(\mathrm{HCl})$ & Merck \\
\hline Hydrogen peroxide $\left(\mathrm{H}_{2} \mathrm{O}_{2}\right)$ & Merck \\
\hline Isoamylalcohol & Merck \\
\hline Isopropanol & Merck \\
\hline Magnesium chloride $\left(\mathrm{MgCl}_{2}\right)$ & Merck \\
\hline Mayer's hemalum solution & Merck Millipore \\
\hline MLV-RT Buffer & Promega \\
\hline RT Buffer & Thermo Scientific \\
\hline Paraffin & Carl Roth \\
\hline PCR Buffer 10X & NEB \\
\hline Random Primer & Promega \\
\hline Random Hexamers & Thermo Scientific \\
\hline Roti-Histokitt & Carl Roth \\
\hline Roti-Phenol & Carl Roth \\
\hline Sodium carbonate $\left(\mathrm{Na}_{2} \mathrm{CO}_{3} / \mathrm{NaHCO}_{3}\right)$ & Merck \\
\hline Sodium chloride $(\mathrm{NaOH})$ & Carl Roth \\
\hline Streptavidin-horseradish peroxidase (HRP) & BioLegend \\
\hline Tris & Carl Roth \\
\hline TRIzol reagent & Invitrogen \\
\hline TRI reagent & Ambion \\
\hline UltraPure Agarose & Invitrogen \\
\hline Xylol & Carl Roth \\
\hline
\end{tabular}




\subsubsection{Consumables}

Table 2.4: List of consumables.

Label

\begin{tabular}{ll}
\hline \hline 96 - well plates for qPCR & Applied Biosystems \\
\hline 96 - well plates for PCR & Thermo Scientific \\
\hline Cover slips glass & Roth \\
\hline Cover slips plastic & Sarstedt \\
\hline Cryo tubes & Greiner \\
\hline Microscope slides & Menzel \\
\hline Multipette plus Combitips & Eppendorf \\
\hline Reaction Tubes $(0.2 \mathrm{ml}, 1 \mathrm{ml}, 2 \mathrm{ml})$ & Greiner/Sarstedt \\
\hline Sterile pipettes (1 ml, $2 \mathrm{ml}, 5 \mathrm{ml}, 10 \mathrm{ml}, 25 \mathrm{ml})$ & Greiner \\
\hline Superfrost Plus glass slides & Thermo \\
\hline Top Seal for qPCR plates & Applied Biosystems \\
\hline Wheighing paper & Eppendorf \\
\hline
\end{tabular}

\subsubsection{Commercial Kits}

Table 2.5: List of commercial kits.

Label

ABsolute Blue QPCR SYBR Green Low ROX Mix

\begin{tabular}{ll}
\hline 2X SsoFasr EvaGreen Supermix with Low ROX & Fluidigm \\
\hline 20X DNA Binding Dye Sample Loading Reagent & Fluidigm \\
\hline 2X Assay Loading Reagent & Fluidigm \\
\hline Fluidigm 48.48 Dynamic Arrays & Fluidigm \\
\hline Fluidigm 96.96 Dynamic Arrays & Fluidigm \\
\hline Taqman PreAmp Master Mix & Applied Biosystems \\
\hline
\end{tabular}




\subsubsection{Devices}

Table 2.6: List of devices.

\begin{tabular}{|c|c|c|}
\hline Description & Label & Supplier \\
\hline Agarose gel trays and chambers & Perfect Blue Gel System & Peglab \\
\hline Autoclave & $\begin{array}{l}\text { High pressure steam sterilisator } \\
\text { FVS }\end{array}$ & Integra Biosciences \\
\hline \begin{tabular}{lll|}
$\begin{array}{l}\text { Biological } \\
\text { Centrifuge }\end{array}$ & Safety & Cabinet \\
\end{tabular} & HERASave ${ }^{\circledR}$ & $\begin{array}{l}\text { Thermo } \\
\text { Scientific }\end{array}$ \\
\hline Centrifuge & Multifuge 3 S-R & Heraeus \\
\hline Centrifuge & Mini Centrifuge MCF-2360 & Heraeus \\
\hline Centrifuge & $3 \mathrm{~K} 30$ & LMS Consult \\
\hline Centrifuge & RC 3B Plus & Sigma \\
\hline Centrifuge & $\begin{array}{lrl}\text { Mini } & \text { Centrifuge } & \text { MCF-2360 } \\
\text { Neubauer improved } & \\
\end{array}$ & Sorvall \\
\hline Dispenser & Multipette plus & Eppendorf \\
\hline Freezer & HERA freeze $-80^{\circ} \mathrm{C}$ & Heraeus \\
\hline Freezer & Liebherr Comfort $-20^{\circ} \mathrm{C}$ & Liebherr GmbH \\
\hline Freezer & VIP plus $-150^{\circ} \mathrm{C}$ & $\begin{array}{l}\text { SANYO Electric Co., } \\
\text { Japan }\end{array}$ \\
\hline Imaging devices & UV workbench GelImager & Intas \\
\hline Imaging devices & Chemilux Blot Detection Imager & Intas \\
\hline Incubator shaker Incubators & Unitron-plus & Infors \\
\hline Microscope & LSCM 510 Axioplan 2 & Zeiss \\
\hline Microscope & Leica RM2255 & Leica Biosystems \\
\hline Microwave & HF12M 900W & Siemens \\
\hline Pipettes & Research $^{\circledR} \&$ Reference ${ }^{\circledR}$ & Eppendorf \\
\hline Pipettor & IBS PIPETBOY acu & Integra Biosciences \\
\hline Power supply & EPS-301/-3501 XL & GE Healthcare \\
\hline Scales & ACCULAB Vicon & Sartorius \\
\hline Slide scanner & dotslode - Virtual slide system & Olympus Life Science \\
\hline Spectrophotometer & NanoDrop $^{\mathrm{TM}}$ ND-1000 & $\begin{array}{ll}\text { Thermo } & \text { Fisher } \\
\text { Scientific } & \\
\end{array}$ \\
\hline Thermal Cycler & ABI 7500 Real-Time PCR System & Applied Biosystems \\
\hline Thermal Cycler & MasterCycler epgradient & Eppendorf \\
\hline Thermal Cycler & Fluidigm & Biomark \\
\hline Thermoblock & Thermomixer comfort & Eppendorf \\
\hline Homogenizer & Tissue Lyser LT & Qiagen \\
\hline Vortex & MS1 Minishaker & IKA \\
\hline Water bath & Medingen W6 & $\begin{array}{l}\text { Labortechnik } \\
\text { Medingen }\end{array}$ \\
\hline
\end{tabular}




\subsubsection{Software}

Table 2.7: List of software.

\section{Product}

7500 System SDS Software

MFE primer-2.0

Microsoft Office Professional Plus 2013

NCBI database

GraphPad PRISM

SPSS

$\mathrm{R}$

Real-Time PCR Analysis

\section{Company}

Applied Biosystems

GitHub Inc.

Microsoft

National Center for

Biotechnology

Information (NCBI)

GraphPad Software Inc.

IBM

http://www.R-

project.org

Biomark

\subsubsection{Laboratory Animals}

Table 2.8: List of laboratory animals.

\begin{tabular}{|c|c|c|}
\hline Product & Species & Description \\
\hline C57BL/6 & Mouse & MHC Haplotype: $\mathrm{H} 2^{\mathrm{b}}$ \\
\hline $\mathrm{BALB} / \mathrm{c}$ & Mouse & MHC Haplotype: $\mathrm{H}^{\mathrm{d}}$ \\
\hline PVG.1N & Rat & $\mathrm{RT}^{\mathrm{n}}, \mathrm{CD} 45.1$ \\
\hline PVG.7B & Rat & $\mathrm{RT}^{\mathrm{c}}{ }^{\mathrm{c}}, \mathrm{CD} 45.2$ \\
\hline PVG & Rat & $\mathrm{RT}^{\mathrm{c}}, \mathrm{CD} 45.1$ \\
\hline
\end{tabular}

\subsubsection{Oligonucleotides}

All primers were synthesized by biomers.net GmbH. Primers were designed using primer BLAST from the National Center for Biotechnology Information (NCBI), and verified using MFE 2.0 software. The primers were designed to span exon-exon junctions, if feasible. In case of targets with two or more isoforms the primers were designed to target all transcripts. The sequences for the mouse, rat and human primers have been listed separately below in alphabetical order. 
Table 2.9: Mouse primer pairs for Real-Time PCR.

\begin{tabular}{|c|c|c|c|}
\hline Gene & Primer sequence $5{ }^{`} 3^{`}$ & Accession ID & $\begin{array}{l}\text { Amplicon } \\
\text { (bp) }\end{array}$ \\
\hline Anp32a & $\begin{array}{l}\text { F: CAGGACGCCCTCTGATGTG } \\
\text { R: CCCTGAGATTCTGTTTTCGCTTA }\end{array}$ & NM_009672.3 & 196 \\
\hline Arg1 & $\begin{array}{l}\text { F: ACCTGGCCTTTGTTGATGTCC } \\
\text { R: AGCACCACACTGACTCTTCCATTC }\end{array}$ & NM_007482.3 & 136 \\
\hline Bmprla & $\begin{array}{l}\text { F: GACACTGCCCAGATGATGCT } \\
\text { R: CTGGGCTTTCGGTGAATCCT }\end{array}$ & NM_009758.4 & 158 \\
\hline C1qtnf7 & $\begin{array}{l}\text { F: ATTGTTGTGGAGCAGCTTGGG } \\
\text { R: CAATCATCTTGGGCTCTTTCCTG }\end{array}$ & NM_001135172.1 & 153 \\
\hline Card11 & $\begin{array}{l}\text { F: GGAGGGCCAGCTATGGATGA } \\
\text { R: GGTTGATGTAACGGCTCAGC }\end{array}$ & NM_175362.2 & 106 \\
\hline Ccl4 & $\begin{array}{l}\text { F: CAACACCATGAAGCTCTGCG } \\
\text { R: CAGGAAGTGGGAGGGTCAGA }\end{array}$ & NM_013652.2 & 108 \\
\hline Ccl5 & $\begin{array}{l}\text { F: TTGTCACTCGAAGGAACCGC } \\
\text { R: AGAGCAAGCAATGACAGGGA }\end{array}$ & NM_013653.3 & 141 \\
\hline Cel9 & $\begin{array}{l}\text { F: CССТСТССТTCСТCATTCTTACA } \\
\text { R: AGTCTTGAAAGCCCATGTGAAA }\end{array}$ & NM_011338.2 & 141 \\
\hline Ccr1 & $\begin{array}{l}\text { F: TCTCCATCATCATACAGGAAGCC } \\
\text { R: GGAACTGGTCAGGAATAATAGCT }\end{array}$ & NM_009912.4 & 180 \\
\hline Ccr4 & $\begin{array}{l}\text { F: GCCTGGTTACAAGCGTAGAGATA } \\
\text { R: GAAAGCCAAACTGCACGGAC }\end{array}$ & NM_009916.2 & 107 \\
\hline Ccr5 & $\begin{array}{l}\text { F: GAGACATCCGTTCCCCCTAC } \\
\text { R: AGCTGAGCCGCAATTTGTTTC }\end{array}$ & NM_009917.5 & 143 \\
\hline Cd226 & $\begin{array}{l}\text { F: TCGTTGGAGGGTTAGTTTCAC } \\
\text { R:GCTACCTTACTCTGTTTATCCCTG }\end{array}$ & NM_178687.2 & 130 \\
\hline Cx3cl1 & $\begin{array}{l}\text { F: GCCGCGTTCTTCCATTTG } \\
\text { R: TGGGATTCGTGAGGTCATCTT }\end{array}$ & NM_009142.3 & 96 \\
\hline Cx3cr1 & $\begin{array}{l}\text { F: AGGACACAGCCAGACAAG } \\
\text { R: TCAGGGGAGAAAGCAAG }\end{array}$ & NM_009987.3 & 134 \\
\hline Cxcl10 & $\begin{array}{l}\text { F: TCCCCATCAGCACCATGAAC } \\
\text { R: CATTCTCACTGGCCCGTCAT }\end{array}$ & NM_021274.2 & 140 \\
\hline Cxcl11 & $\begin{array}{l}\text { F:GTTGAAGTGATTGTTACTATGAAG } \\
\text { R: TGGCACAGAGTTCTTATTGG }\end{array}$ & NM_019494.1 & 175 \\
\hline Cxcl15 (Il8) & $\begin{array}{l}\text { F: GAGTATGACGATTCTGCTGAGG } \\
\text { R: CAGACCGAACGTGAAGACGAG }\end{array}$ & NM_011339.2 & 102 \\
\hline Cxcl16 & $\begin{array}{l}\text { F: TCAGGTTCCAGTTGCAGTCC } \\
\text { R: GTCTGGGTACTGGCTTGAGG }\end{array}$ & NM_023158.6 & 154 \\
\hline
\end{tabular}




\begin{tabular}{|c|c|c|c|}
\hline \multirow{2}{*}{ Cxcl9 } & F: AGGCACGATCCACTACAAATC & \multirow{2}{*}{ NM_008599.4 } & \multirow{2}{*}{154} \\
\hline & R: CCATTCTTTCATCAGCTTCTTCAC & & \\
\hline Cxcr3 & $\begin{array}{l}\text { F: GCAAGTTCCCAACCACAAGT } \\
\text { R: TCTCGTTTTCCCCATAATCG }\end{array}$ & NM_009910.2 & 189 \\
\hline Cxcr 4 & $\begin{array}{l}\text { F: GATAGCCTGTGGATGGTGGT } \\
\text { R: GCAGGCAAAGAAAGCTAGGA }\end{array}$ & NM_009911.3 & 180 \\
\hline Enpp1 & $\begin{array}{l}\text { F: CTGGTTTTGTCAGTATGTGTGCT } \\
\text { R: CTCACCGCACCTGAATTTGTT }\end{array}$ & NM_008813.3 & 231 \\
\hline Fcer1g & $\begin{array}{l}\text { F: ATCTCAGCCGTGATCTTGTTCT } \\
\text { R:ACCATACAAAAACAGGACAGCAT }\end{array}$ & NM_010185.4 & 105 \\
\hline Fcgr3 & $\begin{array}{l}\text { F: CAGAATGCACACTCTGGAAGC } \\
\text { R: GGTCCCTTCGCACATCAG }\end{array}$ & NM_010188.5 & 172 \\
\hline Foxp3 & $\begin{array}{l}\text { F: GCGAAAGTGGCAGAGAGGTA } \\
\text { R: CAGAGGCAGGCTGGATAACG }\end{array}$ & NM_001199347.1 & 169 \\
\hline Gapdh & $\begin{array}{l}\text { F: TGTGTCCGTCGTGGATCTGA } \\
\text { R: TTGCTGTTGAAGTCGCAGGAG }\end{array}$ & NM_008084.2 & 150 \\
\hline H60a & $\begin{array}{l}\text { F: CACCCACTTCATCCTCTGTTTCTT } \\
\text { R: CTTTGCCATGGTCTTCCCTCAG }\end{array}$ & NM_010400.2 & 112 \\
\hline Hcls1 & $\begin{array}{l}\text { F: TGTTGGGGAGTTAGATCGGC } \\
\text { R: CGCTTCCCTCTCCTTGGTAA }\end{array}$ & NM_008225.2 & 167 \\
\hline Hprt & $\begin{array}{l}\text { F: GTCCTGTGGCCATCTGCCTA } \\
\text { R: GGGACGCAGCAACTGACATT }\end{array}$ & NM_013556.2 & 91 \\
\hline Htral & $\begin{array}{l}\text { F: CTCCTTCGCAATTCCATCCG } \\
\text { R: GCTTTGCTAGATGTGAGCGA }\end{array}$ & NM_019564.3 & 132 \\
\hline Icam1 & $\begin{array}{l}\text { F: AGCCTCCGGACTTTCGATCT } \\
\text { R: TGTTTGTGCTCTCCTGGGTC }\end{array}$ & NM_010493.2 & 175 \\
\hline Ido1 & $\begin{array}{l}\text { F: GCTTCTTCCTCGTCTCTCTATTG } \\
\text { R: CTTTCAGGTCTTGACGCTCTAC }\end{array}$ & NM_008324.1 & 99 \\
\hline Ifng & $\begin{array}{l}\text { F: GCTACACACTGCATCTTGGC } \\
\text { R: GCCAGTTCCTCCAGATATCCA }\end{array}$ & NM_008337.3 & 166 \\
\hline Illo & $\begin{array}{l}\text { F: CTAACCGACTCCTTAATGC } \\
\text { R: AATCACTCTTCACСTGCTC }\end{array}$ & NM_010548.2 & 241 \\
\hline Il12a & $\begin{array}{l}\text { F: CCAGGTGTCTTAGCCAGTC } \\
\text { R: CTCGTTCTTGTGTAGTTCCAG }\end{array}$ & NM_001159424.1 & 179 \\
\hline$I l 13$ & $\begin{array}{l}\text { F: AAAGCAACTGTTTCGCCACG } \\
\text { R: CCTCTCCCCAGCAAAGTCTG }\end{array}$ & NM_008355.3 & 107 \\
\hline$I l 15$ & $\begin{array}{l}\text { F: ACATCCATCTCGTGCTACTTGT } \\
\text { R: GCCTCTGTTTTAGGGAGACCT }\end{array}$ & NM_008357.2 & 113 \\
\hline$I l 17 a$ & $\begin{array}{l}\text { F: AGGCAGCAGCGATCATCC } \\
\text { R: GTGGAACGGTTGAGGTAGTC }\end{array}$ & NM_010552.3 & 151 \\
\hline Illr1 & $\begin{array}{l}\text { F: GTGCTACTGGGGCTCATTTGT } \\
\text { R: GGAGTAAGAGGACACTTGCGAAT }\end{array}$ & NM_008362.2 & 134 \\
\hline
\end{tabular}




\begin{tabular}{|c|c|c|c|}
\hline Illrl2 & $\begin{array}{l}\text { F: GAGTTCACCAGGACCAGACC } \\
\text { R: TTTGGTAGCAGTTGTGGGCA }\end{array}$ & NM_133193.3 & 102 \\
\hline$I l 2$ & $\begin{array}{l}\text { F: TGAGCAGGATGGAGAATTACAGG } \\
\text { R: GTCCAAGTTCATCTTCTAGGCAC }\end{array}$ & NM_008366.3 & 120 \\
\hline$I l 22$ & $\begin{array}{l}\text { F: CAACTTCCAGCAGCCATAC } \\
\text { R: ATCCTTAGCACTGACTCCTC }\end{array}$ & NM_016971.2 & 127 \\
\hline$I l 23 a$ & $\begin{array}{l}\text { F: CTGCTTGACTCTGACATC } \\
\text { R: CACTGCTGACTAGAACTC }\end{array}$ & NM_031252.2 & 161 \\
\hline Il2ra & $\begin{array}{l}\text { F: TTGTCGGGCAGAACTGTGTC } \\
\text { R: TCTTCGGAAACCTCTCTTGCA }\end{array}$ & NM_008367.3 & 118 \\
\hline Il4 & $\begin{array}{l}\text { F: CGAGGTCACAGGAGAAGGGA } \\
\text { R: AAGCACCTTGGAAGCCCTAC }\end{array}$ & NM_021283.2 & 112 \\
\hline Il4ra & $\begin{array}{l}\text { F: TGATACAGCGCCTTCCACTG } \\
\text { R: TGGCTTCGGGTCTGCTTATC }\end{array}$ & NM_001008700.3 & 196 \\
\hline$I l 5$ & $\begin{array}{l}\text { F: CACCGAGCTCTGTTGACAAG } \\
\text { R: CCACACTTCTCTTTTTGGCGG }\end{array}$ & NM_010558.1 & 200 \\
\hline Il6 & $\begin{array}{l}\text { F: GACAAAGCCAGAGTCCTTCAGA } \\
\text { R: CTGGTCCTTAGCCACTCCTTC }\end{array}$ & NM_031168.1 & 156 \\
\hline Klrk1 & $\begin{array}{l}\text { F: GCTGGTTAAGTCCTATCACTGG } \\
\text { R: TTGAGCCATAGACAGCACAG }\end{array}$ & NM_001083322.1 & 143 \\
\hline Lgals3 & $\begin{array}{l}\text { F:GAGCACTAATCAGGAAAATGGCAG } \\
\text { R: CATGCACCCGGATATCCTTGA }\end{array}$ & NM_010705.3 & 94 \\
\hline Lgals 7 & $\begin{array}{l}\text { F: TGAACCACTACCTTGCCTTTAC } \\
\text { R: GGACCATGCCTCGAATTCTC }\end{array}$ & NM_008496.4 & 157 \\
\hline Lilra5 & $\begin{array}{l}\text { F: CGGAAGGGAATCCGCACAA } \\
\text { R: CACCTCACATGAGATGGTCAC }\end{array}$ & NM_001081239.2 & 89 \\
\hline$l l 33$ & $\begin{array}{l}\text { F: ACTGCATGAGACTCCGTTCTG } \\
\text { R: CCTAGAATCCCGTGGATAGGC }\end{array}$ & NM_133775.2 & 137 \\
\hline Lst1 & $\begin{array}{l}\text { F: TGTGCCGGTTCAGTCAGAGA } \\
\text { R: GCGATGCAGGCATAGTCAGT }\end{array}$ & NM_010734.2 & 154 \\
\hline Msr1 & $\begin{array}{l}\text { F: GCACAATCTGTGATGATCGCT } \\
\text { R: CCCAGCATCTTCTGAATGTGAA }\end{array}$ & NM_001113326.1 & 224 \\
\hline Pik3ap1 & $\begin{array}{l}\text { F: GTCCCGGATGCCTCTTTCTC } \\
\text { R: CACAAGTCATTTCCTGCCAGT }\end{array}$ & NM_031376.3 & 241 \\
\hline Pstpip1 & $\begin{array}{l}\text { F: GGCCCACACAGGGTATGAG } \\
\text { R: CAGCCTTGCGTGCAATCTG }\end{array}$ & NM_011193.2 & 143 \\
\hline Ptger2 & $\begin{array}{l}\text { F: TCCCTAAAGGAAAAGTGGGACC } \\
\text { R: GAGCGCATTAACCTCAGGACC }\end{array}$ & NM_008964.4 & 113 \\
\hline Ptpn7 & $\begin{array}{l}\text { F: TAGGTGCTCTGCTTCTTTAGTC } \\
\text { R: CTCCCTTGCACCCTTCTATAC }\end{array}$ & NM_177081.3 & 152 \\
\hline$P v r$ & $\begin{array}{l}\text { F: AGCACGAACACGGGTGACTTT } \\
\text { R: CTAGGGCATTGGTGACTTC }\end{array}$ & NM_027514.2 & 121 \\
\hline
\end{tabular}




\begin{tabular}{|c|c|c|c|}
\hline Pvrl2 & $\begin{array}{l}\text { F: CGAGAGTCACCCAGCACAG } \\
\text { R: GTGTCGGCAGATGAGGATG }\end{array}$ & NM_008990.3 & 114 \\
\hline Rae1 & $\begin{array}{l}\text { F: TGATACCCGAATGCAGACAGG } \\
\text { R: TGGGGGACCTTGAGGTTGAT }\end{array}$ & NM_009016.1 & 81 \\
\hline Tap1 & $\begin{array}{l}\text { F: GGACTTGCCTTGTTCCGAGAG } \\
\text { R: GCTGCCACATAACTGATAGCGA }\end{array}$ & NM_013683.2 & 116 \\
\hline$T g f b 1$ & $\begin{array}{l}\text { F: TTACCTTGGTAACCGGCTGC } \\
\text { R: AGCCCTGTATTCCGTCTCCT }\end{array}$ & NM_011577.1 & 110 \\
\hline$T g f b 2$ & $\begin{array}{l}\text { F: CGAGGCGAGATTTGCAGGTAT } \\
\text { R: GCAGGAGATGTGGGGTCTTC }\end{array}$ & NM_009367.3 & 110 \\
\hline$T g f b r 1$ & $\begin{array}{l}\text { F: CGGTTTGGAGAAGTTTGGCG } \\
\text { R: CGTCCATGTCCCATTGTCTTTG }\end{array}$ & NM_009370.2 & 180 \\
\hline $\operatorname{Tgm} 2$ & $\begin{array}{l}\text { F: GACAATGTGGAGGAGGGATCT } \\
\text { R: CTCTAGGCTGAGACGGTACAG }\end{array}$ & NM_009373.3 & 120 \\
\hline$T n f$ & $\begin{array}{l}\text { F: ACAGAAAGCATGATCCGCGA } \\
\text { R: GACCGATCACCCCGAAGTTC }\end{array}$ & NM_013693.2 & 166 \\
\hline Trem 2 & $\begin{array}{l}\text { F: CCAGTGTCAGAGTCTCCGAG } \\
\text { R: GAGGTCTCTTGATTCCTGGAGG }\end{array}$ & NM_031254.2 & 144 \\
\hline$U b c$ & $\begin{array}{l}\text { F: AGGTCAAACAGGAAGACAGACGTA } \\
\text { R:AGGTCAAACAGGAAGACAGACGTA }\end{array}$ & NM_019639.4 & 80 \\
\hline Ubd & $\begin{array}{l}\text { F: CCAATGGCGGTTAATGACCTT } \\
\text { R: TTTCGATGGGGCTTGAGGATT }\end{array}$ & NM_023137.3 & 141 \\
\hline Ulbp1 & $\begin{array}{l}\text { F: AGACTAACACAACCGGAAAGCC } \\
\text { R: АACCTTGTCTTCCTTGCACATT }\end{array}$ & NM_029975.2 & 200 \\
\hline
\end{tabular}

Table 2.10: Rat primer pairs for Real-Time PCR.

\begin{tabular}{|c|c|c|c|}
\hline Gene & Primer sequence $5-3$ & Accession ID & $\begin{array}{c}\text { Amplico } \\
\text { n (bp) }\end{array}$ \\
\hline$C d 226$ & $\begin{array}{l}\text { F: TGTCTCCTTCTCTTCCTGTTGA } \\
\text { R: TCAGGACTTTACAGATGCCGC }\end{array}$ & NM_001107370.1 & 191 \\
\hline C1qtnf7 & $\begin{array}{l}\text { F: GGTTGTCCACTTGCACAGATG } \\
\text { R: CCTGACTTTTGTACACTGGGAA }\end{array}$ & NM_001107221.1 & 113 \\
\hline Gapdh & $\begin{array}{l}\text { F: GGGCTGCCTTCTCTTCTGAC } \\
\text { R: CGCCAGTAGACTCCACGACA }\end{array}$ & NM_017008.3 & 243 \\
\hline Hcls1 & $\begin{array}{l}\text { F: TCTTATATGCCGTGGTTGGGG } \\
\text { R: TCAGCGAGATTATGCCAAGGG }\end{array}$ & NM_001011898.1 & 113 \\
\hline Hprt & $\begin{array}{l}\text { F: GCTGAAGATTTGGAAACAGGTG } \\
\text { R: AATCCAGCAGGTCAGCAAAG }\end{array}$ & NM_012583.2 & 122 \\
\hline Htral & $\begin{array}{l}\text { F: TGACAGTTTTCCCTTTGGCCTG } \\
\text { R: TTCAGACAGACGCCATCATCAA }\end{array}$ & NM_031721.1 & 183 \\
\hline
\end{tabular}




\begin{tabular}{|c|c|c|c|}
\hline Klrk1 & $\begin{array}{l}\text { F: TGTTCGAGTCCTTGTTGCAG } \\
\text { R: AGCAGGCTGGAATTTTGAGA }\end{array}$ & NM_133512.1 & 240 \\
\hline Lgals 7 & $\begin{array}{l}\text { F: CATCCACAACCCTCCTCTACG } \\
\text { R: TTCAAGACTGTGATCGGGGAT }\end{array}$ & NM_022582.2 & 187 \\
\hline Lilra5 & $\begin{array}{l}\text { F: AAAAGACGGTATTCCTGGGCC } \\
\text { R: TGACCTTCATCTTCACAGCCG }\end{array}$ & NM_001076793.1 & 181 \\
\hline Lst 1 & $\begin{array}{l}\text { F: GGGCAGGAGCTCCACTACG } \\
\text { R: CGATGCAGGCATAGTCAGTGC }\end{array}$ & NM_022634.2 & 118 \\
\hline Msr1 & $\begin{array}{l}\text { F: GTCCAGCTCGTATATCCCAGC } \\
\text { R: AAGGCAGGGAGGTCAGGATTT }\end{array}$ & NM_001191939.1 & 190 \\
\hline Pi3kap1 & $\begin{array}{l}\text { F: TTTCTGCCCCACAACGAATG } \\
\text { R: TGTGATGACGAACCGGAGAC }\end{array}$ & NM_001106368.1 & 199 \\
\hline Pstpip1 & $\begin{array}{l}\text { F: TCTCCCGCAGAAATGTCCAG } \\
\text { R: CTGCCGCTTCCACAGAGACT }\end{array}$ & NM_001106824.2 & 172 \\
\hline Ptger2 & $\begin{array}{l}\text { F: AGGATGACAAAAACCCAAGGATC } \\
\text { R: TTCTATGGCGGAGGAGACGG }\end{array}$ & NM_031088.1 & 186 \\
\hline Ptpn7 & $\begin{array}{l}\text { F: CAGGAAGTGCAGGGTGACTT } \\
\text { R: TCTTTGGGGGCAGACATGAC }\end{array}$ & NM_145683.1 & 177 \\
\hline$P v r$ & $\begin{array}{l}\text { F: ACCACAACCACGCCTGTATTT } \\
\text { R: TCTGAGATTCTGCCGCCAAA }\end{array}$ & NM_017076.2 & 123 \\
\hline Pvrl2 & $\begin{array}{l}\text { F: GTGAGGGCATTTCTGGTTCCT } \\
\text { R: ATCATTGGAGGCATCATCGCT }\end{array}$ & NM_001012064.1 & 187 \\
\hline Raet1 & $\begin{array}{l}\text { F: CCTCTCCGGTATGAAGGACA } \\
\text { R: CCTTAAGTCCTGGCCCAACAG }\end{array}$ & NM_001013063.1 & 165 \\
\hline Rrlt & $\begin{array}{l}\text { F: GCATCCTCTATTCACAGCAGC } \\
\text { R: CCCTTAAGTCCTGTTTACATC }\end{array}$ & NM_001161691.1 & 180 \\
\hline Tap1 & $\begin{array}{l}\text { F: CTCGGGGCTCTCATACAGGA } \\
\text { R: TCATCTCTGGATTCCCTCAGG }\end{array}$ & NM_032055.4 & 200 \\
\hline $\operatorname{Tgm} 2$ & $\begin{array}{l}\text { F: ACAAGACTCACGGTGCGATT } \\
\text { R: TACTCCTACCCCTCTGTGGC }\end{array}$ & NM_019386.2 & 180 \\
\hline Trem 2 & $\begin{array}{l}\text { F: TACAGACGTTTACCAGCAACCA } \\
\text { R: CCACCAAGACTTCTGTTCTGCTA }\end{array}$ & NM_001106884.1 & 142 \\
\hline Ubd & $\begin{array}{l}\text { F: TGGGGTGATGAGAAGCTCAAAA } \\
\text { R:CCCCACСТCAAATCTTTATTTCATTC }\end{array}$ & NM_053299.2 & 105 \\
\hline
\end{tabular}

Table 2.11: Human primer pairs for Real-Time PCR.

\begin{tabular}{|c|l|c|c|}
\hline Gene & \multicolumn{1}{|c|}{ Primer sequence 5 - 3 } & Accession ID & $\begin{array}{c}\text { Amplicon } \\
\text { (bp) }\end{array}$ \\
\hline \multirow{2}{*}{$\boldsymbol{A N P 3 2 A}$} & $\begin{array}{l}\text { F: ATGAACTGCTAGGTGAGGGG } \\
\text { R: GATACACCACTGAGTCTTGCTTT }\end{array}$ & NM_006305.3 & 128 \\
\hline
\end{tabular}




\begin{tabular}{|c|c|c|c|}
\hline BMPR1A & $\begin{array}{l}\text { F: GATTCGGGCCCCACTTCG } \\
\text { R: ACAGCTTTGACCTATGCTTCCA }\end{array}$ & NM_004329.2 & 155 \\
\hline C1QTNF7 & $\begin{array}{l}\text { F: TTTGCATTGTTTTGGAGTAGCT } \\
\text { R: ACATCTTTGGCTCTTGTCTGGA }\end{array}$ & NM_001135170.1 & 156 \\
\hline CARD11 & $\begin{array}{l}\text { F: GCCCATCACCAACTCCTTCA } \\
\text { R: TTCTTCGACTGTGCTGCGAT }\end{array}$ & NM_032415.5 & 142 \\
\hline CD226 & $\begin{array}{l}\text { F: TCCTGCAGTGCTAATTAAGGGAGG } \\
\text { R: AAAGGCTGGTTCTTGAGATGTGA }\end{array}$ & NM_006566.2 & 168 \\
\hline CCL4 & $\begin{array}{l}\text { F: ACTCCTCTCCGCAGTTCCT } \\
\text { R: ACAGAATCAAATGTGTTATCCATGT }\end{array}$ & NM_002984.2 & 184 \\
\hline CCL5 & $\begin{array}{l}\text { F: CGAAAGAACCGCCAAGTGTG } \\
\text { R: CGGGTGGGGTAGGATAGTGA }\end{array}$ & NM_002985.2 & 177 \\
\hline CCRI & $\begin{array}{l}\text { F: TTGGAACCAGAGAGAAGCCG } \\
\text { R: ACCAAGGAGTACAGAGGGGG }\end{array}$ & NM_001295.2 & 153 \\
\hline CCR4 & $\begin{array}{l}\text { F: CGGGTCCTTCTTAGCATCGT } \\
\text { R: GCCCAACCAGAAGCAGCTTG }\end{array}$ & NM_005508.4 & 100 \\
\hline CCR5 & $\begin{array}{l}\text { F: TCCAGTGAGAAAAGCCCGTAA } \\
\text { R: GGCGAAAAGAATCAGAGAACAGT }\end{array}$ & NM_000579.3 & 105 \\
\hline CX3CR1 & $\begin{array}{l}\text { F: GAAAGAAAACTGCTGGTGGACT } \\
\text { R: GATCCATGGTGAAGGCCTCTAGT }\end{array}$ & NM_001171171.1 & 180 \\
\hline CXCR3 & $\begin{array}{l}\text { F: CGAGAGAAGCAGCCTTTGAGAAG } \\
\text { R: TATAACTGTCCCCGCCAGTC }\end{array}$ & NM_001142797.1 & 104 \\
\hline CXCR4 & $\begin{array}{l}\text { F: GAGGAGTTAGCCAAGATGTGACT } \\
\text { R: TCCCCATCTTTTCCCATAGTGAC }\end{array}$ & NM_001008540.1 & 128 \\
\hline CXCL8 & 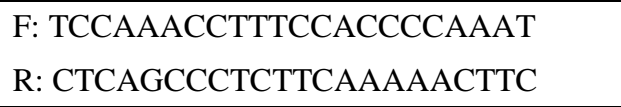 & NM_000584.3 & 171 \\
\hline CXCL9 & $\begin{array}{l}\text { F: CTCTGCAGGATGTGGAAACCT } \\
\text { R: GATAAGACGTTCGGGTGGGA }\end{array}$ & NM_002416.2 & 170 \\
\hline CXCL10 & $\begin{array}{l}\text { F: ACACTAGCCCCACGTTTTCT } \\
\text { R: GGTACTCCTTGAATGCCACT }\end{array}$ & NM_001565.3 & 154 \\
\hline CXCL11 & $\begin{array}{l}\text { F: TGTGAAGGATGAAAGGTGGGT } \\
\text { R: AATTGTTGGACTCCTTTGGGC }\end{array}$ & NM_005409.4 & 100 \\
\hline CXCL16 & $\begin{array}{l}\text { F: CACCGCAGTCCTCTCCAGATC } \\
\text { R: AGCCACAGTTTACCCTCACAA }\end{array}$ & NM_001100812.1 & 101 \\
\hline ENPP1 & $\begin{array}{l}\text { F: AGACCACACTTTTACACTCTGT } \\
\text { R: CTGCCTTGTTCCATGCCATG }\end{array}$ & NM_006208.2 & 200 \\
\hline FCER1G & $\begin{array}{l}\text { F: CTGAAGATCCAAGTGCGAAAGG } \\
\text { R: CTGTGGTGGTTTCTCATGCTTC }\end{array}$ & NM_004106.1 & 123 \\
\hline FCGR3 & $\begin{array}{l}\text { F: TGACAGAGATGGGTGGAGGG } \\
\text { R: TTGGGAGATCTTCAGTCCGC }\end{array}$ & NM_000569.6 & 189 \\
\hline GAPDH & $\begin{array}{l}\text { F: TTCGACAGTCAGCCGCATC } \\
\text { R: GCCCAATACGACCAAATCCGT }\end{array}$ & NM_002046.5 & 105 \\
\hline HCLS1 & $\begin{array}{l}\text { F: ATTACTCTCGTGGCTTTGGTGG } \\
\text { R: CCCTTGGCATAATCTCTCTGGG }\end{array}$ & NM_005335.4 & 124 \\
\hline
\end{tabular}




\begin{tabular}{|c|c|c|c|}
\hline HPRT & $\begin{array}{l}\text { F: TGGTCAGGCAGTATAATCCAAAGA } \\
\text { R:GTCAAGGGCATATCCTACAACAAAC }\end{array}$ & NM_000194.2 & 137 \\
\hline HTRA1 & $\begin{array}{l}\text { F: TCCGAATGATGTCACTCACGT } \\
\text { R: GTTTTCCTTGAGACCACCAGCT }\end{array}$ & NM_002775.4 & 140 \\
\hline ICAMI & $\begin{array}{l}\text { F: TGACCATCTACAGCTTTCCGG } \\
\text { R: CAGCGTCACCTTGGCTCTAG }\end{array}$ & NM_000201.2 & 113 \\
\hline ILIRI & $\begin{array}{l}\text { F: AGGGATGACTACGTTGGGGA } \\
\text { R: TTCAGATGAACCACCCAGCC }\end{array}$ & NM_000877.2 & 126 \\
\hline$I L 1 R 2$ & $\begin{array}{l}\text { F: CCCACATAGAGAGCGCCTAC } \\
\text { R: AAGAGCGAAACCCACAGAGT }\end{array}$ & NM_001261419.1 & 100 \\
\hline$I L 2$ & $\begin{array}{l}\text { F: CAAGAATCCCAAACTCACCAGG } \\
\text { R: ATTGCTGATTAAGTCCCTGGGTC }\end{array}$ & NM_000586.3 & 172 \\
\hline$I L 2 R A$ & $\begin{array}{l}\text { F: GCTCTGCCACTCGGAACACA } \\
\text { R: TCTGCCCCACCACGAAATGA }\end{array}$ & NM_000417.2 & 189 \\
\hline IL4 & $\begin{array}{l}\text { F: ACATCTTTGCTGCCTCCAAGAAC } \\
\text { R: AGGAATCGGATCAGCTGCTTG }\end{array}$ & NM_000589.2 & 157 \\
\hline$I L 4 R$ & $\begin{array}{l}\text { F: GTCTCCGCGCCCAGGAAA } \\
\text { R: CTGCTCCATTCGCCGTCC }\end{array}$ & NM_000418.3 & 100 \\
\hline IL5 & $\begin{array}{l}\text { F: GCTGCCTACGTGTATGCCA } \\
\text { R: CCAGTGTGCCTATTCCCTGAA }\end{array}$ & NM_000879.2 & 184 \\
\hline IL6 & $\begin{array}{l}\text { F: CAATATTAGAGTCTCAACCCCCA } \\
\text { R: CTGGAGGGGAGATAGAGCTTCT }\end{array}$ & NM_000600.3 & 107 \\
\hline IL10 & $\begin{array}{l}\text { F: TGCCAAGCCTTGTCTGAGAT } \\
\text { R: ATCGATGACAGCGCCGTAG }\end{array}$ & NM_000572.2 & 148 \\
\hline IL13 & $\begin{array}{l}\text { F: TTTCGCGAGGGACAGTTCAA } \\
\text { R: CCCGCCTACCCAAGACATTT }\end{array}$ & NM_002188.2 & 112 \\
\hline IL15 & $\begin{array}{l}\text { F: GTCTTCATTTTGGGCTGTTTCA } \\
\text { R: CTTTGCAACTGGGGTGAACATC }\end{array}$ & NM_000585.4 & 157 \\
\hline KLRK1 & $\begin{array}{l}\text { F: CAAGATCTTCCCTCTCTGAGCAG } \\
\text { R: CTCATCTCCCAGCTGTGTCGA }\end{array}$ & NM_007360.3 & 135 \\
\hline LGALS7 & $\begin{array}{l}\text { F: CCCAGCCATGTCCAACGT } \\
\text { R: TGTTGAAGACCACCTCCGAC }\end{array}$ & NM_002307.3 & 197 \\
\hline LILRA5 & $\begin{array}{l}\text { F: CAGCCAGGTGTCAGATGTGT } \\
\text { R: GCCCAGACTCAGCCCGA }\end{array}$ & NM_021250.3 & 200 \\
\hline LST1 & $\begin{array}{l}\text { F: GCCCCTGATCATTTCGCCTAA } \\
\text { R: CAGCCTCTTTACATCATTCCGC }\end{array}$ & NM_001166538.1 & 105 \\
\hline MICA & $\begin{array}{l}\text { F: ACTTGACAGGGAACGGAAAGGA } \\
\text { R: CCATCGTAGTAGAAATGCTGGGA }\end{array}$ & NM_001177519.1 & 148 \\
\hline$M I C B$ & $\begin{array}{l}\text { F: ATCTGTGCAGTCAGGGTTTCTC } \\
\text { R: TGAGGTCTTGCCCATTCTCTGT }\end{array}$ & NM_005931.3 & 173 \\
\hline MSR1 & $\begin{array}{l}\text { F: CGTCTGTAGGAGCTTGGGATA } \\
\text { R: AAAGTGCAAGTGACTCCAGC }\end{array}$ & NM_138715.2 & 195 \\
\hline PIK3AP1 & $\begin{array}{l}\text { F: GAGCTTTCCATCCTCCGCAC } \\
\text { R: AGTCACAGCCAGAATCTTCGG }\end{array}$ & NM_152309.2 & 177 \\
\hline PSTPIP1 & F: GGCCTCCTTTGACTCCTTGA & NM_003978.3 & 145 \\
\hline
\end{tabular}




\begin{tabular}{|c|c|c|c|}
\hline & R: GACGGCCTCATACTTCTTCCT & & \\
\hline PTGER2 & $\begin{array}{l}\text { F: CTCCTTGCCTTTCACGATTTTT } \\
\text { R: ACGCATTAGTCTCAGAACAGGA }\end{array}$ & NM_000956.3 & 154 \\
\hline PTPN7 & $\begin{array}{l}\text { F: CTTCAAACTTTGTCAGCCCCG } \\
\text { R: CGTCATAGCCTCGGATGTAGT }\end{array}$ & NM_002832.3 & 165 \\
\hline$P V R$ & $\begin{array}{l}\text { F: GCTCTGCTGTTTGTTCTGCTTTCC } \\
\text { R: TTTCTGCTGCTGGATGCGGTTT }\end{array}$ & NM_006505.3 & 165 \\
\hline PVRL2 & $\begin{array}{l}\text { F: TGGACTGGGAAGCCAAAGAGA } \\
\text { R:TACAGAGAGGGTCACAGGTATCAGG }\end{array}$ & NM_001042724.1 & 173 \\
\hline TAPI & $\begin{array}{l}\text { F: TGGTCCTCTCCTCTCTTGGGG } \\
\text { R: GTTGTTATAGATCCCGTCACCCA }\end{array}$ & NM_000593.5 & 173 \\
\hline TGFB1 & $\begin{array}{l}\text { F: CAGCAACAATTCCTGGCGATA } \\
\text { R: ATTTCCCCTCCACGGCTCAA }\end{array}$ & NM_000660.4 & 120 \\
\hline$T G F B 2$ & $\begin{array}{l}\text { F: CTTCCCCTCCGAAACTGTCTG } \\
\text { R: CATCAAGGTACCCACAGAGCA }\end{array}$ & NM_001135599.2 & 101 \\
\hline TGFBRI & $\begin{array}{l}\text { F: CCGTTTGTATGTGCACCCTC } \\
\text { R: AATGACAGCTGCCAGTTCCA }\end{array}$ & NM_004612.2 & 144 \\
\hline$T G M 2$ & $\begin{array}{l}\text { F: GATCCAGGGTGACAAGAGCG } \\
\text { R: CCACAGCAGTACGTCCCTTC }\end{array}$ & NM_004613.2 & 150 \\
\hline TREM2 & $\begin{array}{l}\text { F: CAGCATCTCCAGGAGCCTCT } \\
\text { R: TGTCCCTGGCTTCTGTCCAT }\end{array}$ & NM_018965.2 & 145 \\
\hline$U B D$ & $\begin{array}{l}\text { F: TGGACAAACACAGCAATCCAG } \\
\text { R: GGAACGGACATGCACACAGAG }\end{array}$ & NM_006398.3 & 150 \\
\hline$U L B P 1$ & $\begin{array}{l}\text { F: TGGGTATCATGCTTACTGTCTGGG } \\
\text { R: GGGTTTGGGTTCATAGTGCAGAGTT }\end{array}$ & NM_025218.2 & 211 \\
\hline ULBP2 & $\begin{array}{l}\text { F: TGGGTATCATGCTTACTGTCTGGG } \\
\text { R: GGGTTTGGGTTCATAGTGCAGAGTT }\end{array}$ & NM_025217.2 & 138 \\
\hline ULBP3 & $\begin{array}{l}\text { F: GCGATTCTTCCGTACCTGCTATTC } \\
\text { R: ACCTGGCTCTGGACCTCACAC }\end{array}$ & NM_024518.1 & 131 \\
\hline VCAM1 & $\begin{array}{l}\text { F: TTTGGATAATGTTTGCAGCTTCTC } \\
\text { R: ATTCGTCACCTTCCCATTCAGT }\end{array}$ & NM_001078.3 & 176 \\
\hline
\end{tabular}




\subsection{Methods}

\subsubsection{Animal aGvHD models}

\subsubsection{Mouse aGvHD model}

The mouse experiment have been done by Prof. Reichardts research group, at the Institute of Cellular and Molecular Immunology, UMG in Göttingen, Germany (Theiss-Suennemann et al., 2015). All interventions were approved by the authorities of Lower Saxony and BadenWürttemberg. C57BL/6 (B6, H-2 $\left.{ }^{\mathrm{b}}\right)$ and BALB/c $\left(\mathrm{H}-2^{\mathrm{d}}\right)$ were purchased from Charles River (Sulzfeld, Germany). We obtained the biopsies from the sacrificed mice for gene expression studies.

\section{Preparation of bone marrow (BM)}

$\mathrm{BM}$ was isolated from the femur and tibia of C57BL/6 mice, and $\mathrm{T}$ cells were depleted using antiCD90.2 microbeads and autoMACS separator (Miltenyl Biotech, Bergisch Gladbach, Germany) or by incubation with anti-Thy-1.2 antibodies followed by lysis with TOX-M rabbit complement (Cederlane, Burlington, CA).

\section{Purification of T cells}

The $\mathrm{T}$ cells were isolated from the spleen and lymph nodes of C57BL/6 mice using $40 \mu \mathrm{m}$ cell strainers. Sorting was done with the Pan T cell isolation Kit II and the autoMACs system (Miltenyi).

\section{MHC-mismatched aGvHD mouse model}

16 hours prior to transplantation, the BALB/c mice were subjected to total body irradiation with a dose of 8.5 Gy and injected via the tail vein with either $1 \times 10^{7} \mathrm{~T}$ cell-depleted (TCD) BM cells or $2 \times 10^{6}$ purified $\mathrm{T}$ cells from C57BL/6 mice. $25 \mu \mathrm{g} / \mathrm{ml}$ neomycin was provided to the mice via the drinking water from day -1 to 28 . The mice were treated i.p. with PBS from day 3 to 6 . The severity of disease was assessed using a five parameter scoring system: posture, activity, ruffling of the fur, diarrhea and loss of weight. Each parameter was assigned an increasing score from 0 to 2, with increase in severity of the symptoms. Mice that had severe weight loss or had scores greater than 7 were euthanized for ethical reasons. 


\subsubsection{2 aGvHD rat model}

\section{Animals and ethical considerations}

The rat aGvHD model was done by Prof. Rolstads research group, at the University of Oslo (UIO), Norway. All the animal experiments were approved by the Norwegian Animal Research Authority under the Ministry of Agriculture of Norway, license number 6060, and conducted in conformity with "the Norwegian Regulations on Animal Experimentation" and "The European Convention for the Protection of Vertebrate Animals used for Experimental and other Scientific Purposes".

During my secondment to UIO, Norway, I participated in the in vivo rat experiments. We received the rat aGvHD biopsies from UIO to perform gene expression analysis.

\section{Preparation of bone marrow}

Bone marrow was isolated from rat tibia and femur and depleted of T cells using anti-CD5 (OX19) and anti-T cell receptor (TCR) $\alpha \beta$ antibodies and anti-mouse IgG coated Dynabeads.

\section{Purification of T cells}

Cells were isolated from cervical and mesenteric lymph node cells (approx. $\sim 60 \% \mathrm{~T}$ cells), and infused into the recipients 14 days after transplant.

\section{MHC-congenic rat aGvHD model}

T cell-depleted bone marrow from PVG.7B rats was transplanted into recipient PVG.1N or PVG rats, previously irradiated with $8.5 \mathrm{~Gy}$ and subsequently injected i.v. with $30 \times 10^{6} \mathrm{~T}$ cell-depleted bone marrow. To induce aGvHD, $1 \times 10^{7}$ cervical and mesenteric lymph node cells from PVG.7B rats were infused into recipients 14 days post transplantation. Weight and GvHD symptoms (activity, kyphosis, skin, and fur integrity) were assessed twice a week. Rats with severe weight loss or evident signs of disease were sacrificed.

\subsubsection{Molecular biology methods}

\subsubsection{RNA preparation}

Total RNA was isolated from the different tissues. 2-5 mm length of snap frozen tissue was homogenized in $1 \mathrm{ml}$ Trizol reagent using a homogenizer. $200 \mu \mathrm{l}$ chloroform was added for phase separation. The colorless upper phase containing the RNA was transferred to a new reaction tube, to 
which $500 \mu \mathrm{l}$ isopropanol was added to precipitate the RNA. The RNA pellet was then washed with $75 \%$ ethanol and air-dried. The pellet was then dissolved in $50 \mu 1$ nuclease-free water and cleaned up for contaminants.

\section{DNA clean up}

To eliminate protein contaminants from the RNA, a cleanup step was carried out. To each of the 50 $\mu 1$ of RNA, the following reagents were added, and incubated at room temperature.

$\begin{array}{ll}6 \mu \mathrm{l} & \text { DNAse I buffer } \\ 1 \mu \mathrm{l} & \text { DNAse I }(10 \mathrm{U} / \mu \mathrm{l}) \\ 1 \mu \mathrm{l} & \text { RNAse Inhibitor }(40 \mathrm{U} / \mu \mathrm{l})\end{array}$

Subsequently, $100 \mu 1$ phenol-chloroform-isoamylalcohol $(25: 24: 1)$ was added to the RNA. The clean phase containing the RNA was transferred to a new reaction tube with $150 \mu \mathrm{l}$ isopropanol and $15 \mu \mathrm{l}$ NaOAc. Following this, the RNA was washed twice with $75 \%$ Ethanol, air-dried and dissolved in $20 \mu \mathrm{l}$ nuclease-free water. The RNA was stored in $-80{ }^{\circ} \mathrm{C}$. The quality of RNA was checked by determining the integrity of the $28 \mathrm{~S}$ and $18 \mathrm{~S}$ RNA by loading RNA on a $1 \%$ agarose gel. The RNA was quantified with a spectrophotometer, Nanodrop 2000 at a wavelength of $260 \mathrm{~nm}$.

\subsubsection{2 cDNA synthesis}

Transcription of RNA into cDNA was carried out by cDNA synthesis kit from Thermo Scientific. To $200 \mathrm{ng}$ RNA, $1.75 \mu \mathrm{l}$ random hexamers were added, and each reaction tube was filled up to a final volume of $15 \mu \mathrm{l}$ with nuclease-free water. The tubes were incubated at $70{ }^{\circ} \mathrm{C}$ for $10 \mathrm{~min}$ to break up RNA secondary structures. The RNA was placed immediately on ice to prevent reforming of these structures. Following this, the following reagents were added to each reaction tube:

$\begin{array}{ll}5 \mu \mathrm{l} & \text { 5X Reverse Transcriptase Buffer } \\ 1 \mu \mathrm{l} & \text { Reverse Transcriptase }(200 \mathrm{U} / \mu \mathrm{l}) \\ 2 \mu \mathrm{l} & \text { dNTPs }(10 \mathrm{mM}) \\ 1 \mu \mathrm{l} & \text { RNAse Inhibitor }(40 \mathrm{U} / \mu \mathrm{l}) \\ 1 \mu \mathrm{l} & \text { DTT }(100 \mathrm{mM})\end{array}$


The reaction tubes were subsequently incubated at $42{ }^{\circ} \mathrm{C}$ for $1 \mathrm{hr}$ for the transcription of RNA into cDNA. The cDNA was stored at $-20^{\circ} \mathrm{C}$.

\subsubsection{Polymerase Chain Reaction (PCR)}

PCR was used to check the specificity of the primers and size of amplified product. The reaction includes 3 steps; denaturation of the double-stranded DNA (dsDNA), annealing of the primer to the single-stranded DNA (ssDNA) template followed by the elongation of the ssDNA by a DNA polymerase.

The PCR reaction was set up as follows:

$\begin{array}{ll}2.5 \mu \mathrm{l} & \text { 10X Taq PCR Buffer } \\ 0.2 \mu \mathrm{l} & \text { Taq DNA polymerase } \\ 0.5 \mu \mathrm{l} & \text { dNTP mix }(10 \mathrm{mM}) \\ 0.5 \mu \mathrm{l} & \text { sense primer }(10 \mathrm{pmol} / \mu \mathrm{l}) \\ 0.5 \mu \mathrm{l} & \text { anti-sense primer }(10 \mathrm{pmol} / \mu \mathrm{l}) \\ 1 \mu \mathrm{l} & \text { DNA template }(200 \mathrm{ng}) \\ \text { up to } 25 \mu \mathrm{l} & \mathrm{dH}_{2} 0\end{array}$

The denaturation and elongation temperatures were set in the PCR program, based on the manufacturer's instructions. Annealing temperatures were set according to the specific melting temperature of the primer pair used. The PCR program is indicated below. 


\begin{tabular}{lcc}
\hline Initial denaturation & $95^{\circ} \mathrm{C}$ & $5 \mathrm{~min}$ \\
\hline Denaturation* & $95^{\circ} \mathrm{C}$ & $30 \mathrm{sec}$ \\
\hline Primer annealing* & $57.5^{\circ} \mathrm{C}$ & $25 \mathrm{sec}$ \\
\hline Elongation* & $72{ }^{\circ} \mathrm{C}$ & $30 \mathrm{sec}$ \\
\hline Final elongation & $72^{\circ} \mathrm{C}$ & $5 \mathrm{~min}$ \\
\hline
\end{tabular}

*in 30 cycles

\subsubsection{Quantitative real-time PCR (qRT-PCR)}

qRT-PCR was performed to determine the relative amount of specific mRNA transcripts of the gene of interest as well as the product specificity of the designed primers (listed in section 2.1.9). The Absolute Blue QPCR SYBR green mix, comprising of the polymerase buffer, dNTPs, a hot start polymerase and a green fluorescent dye, was used to amplify and detect the DNA respectively. For the $\mathrm{qPCR}$, the cDNA was diluted 1:4 with $\mathrm{dH}_{2} 0$ and the following reagents were added per reaction:

$\begin{array}{ll}10 \mu \mathrm{l} & \text { Absolute Blue QPCR SYBR green mix } \\ 0.5 \mu \mathrm{l} & \text { sense primer }(10 \mathrm{pmol} / \mu \mathrm{l}) \\ 0.5 \mu \mathrm{l} & \text { anti-sense primer }(10 \mathrm{pmol} / \mu \mathrm{l}) \\ 8 \mu \mathrm{l} & \mathrm{dH}_{2} 0 \\ 1 \mu \mathrm{l} & \text { template cDNA (1:4) }\end{array}$


The qRT-PCR program used is indicated below:

\begin{tabular}{llc} 
Step & Temperature & Time \\
\hline \hline Enzyme activation & $50{ }^{\circ} \mathrm{C}$ & $2 \mathrm{~min}$ \\
\hline Initial denaturation* & $95^{\circ} \mathrm{C}$ & $10 \mathrm{~min}$ \\
\hline Denaturation* & $95^{\circ} \mathrm{C}$ & $15 \mathrm{sec}$ \\
\hline Annealing/ elongation* & $60^{\circ} \mathrm{C}$ & $15 \mathrm{~min}$ \\
\hline Dissociation stage & $95^{\circ} \mathrm{C}$ & $20 \mathrm{sec}$ \\
\hline & $60{ }^{\circ} \mathrm{C}$ & $20 \mathrm{sec}$ curve \\
\hline Recording of the dissociation & stepwise rising of the & \\
\hline
\end{tabular}

*in 40 cycles

\subsubsection{Gene expression using Biomark Fluidigm dynamic Arrays}

\subsubsection{Preamplification of cDNA}

\section{Pooled STA (Specific Target Amplification) Master Mix}

The pooled STA master mix comprised of $100 \mu \mathrm{M}$ of each primer pair (up to 96 primer pairs) mixed together for the preamplification of specific products on each template. DNA suspension buffer was added to the master mix to make the volume upto $200 \mu 1$.

$1 \mu \mathrm{l}$ of each primer pair $\quad 100 \mu \mathrm{M}$ Primer Stock (up to 96 primer pairs)

upto $200 \mu \mathrm{l} \quad$ DNA Suspension Buffer (10mM Tris, pH 8.0, 0.1M EDTA) 
Premaplification sample pre-mix

\begin{tabular}{|c|c|c|c|c|}
\hline \multicolumn{2}{|r|}{ Component } & $\begin{array}{c}\text { Volume/ } \\
\text { reaction tube } \\
(\mu \mathrm{l})\end{array}$ & $\begin{array}{c}\text { Volume for } 48 \\
\text { reactions + } \\
\text { overage }(\mu \mathrm{l})\end{array}$ & $\begin{array}{c}\text { Volume for } 96 \\
\text { reactions + } \\
\text { overage }(\mu \mathrm{l})\end{array}$ \\
\hline \multirow{3}{*}{ Pre-mix } & $\begin{array}{ll}\text { Taqman } & \text { PreAmp } \\
\text { Master Mix } & \end{array}$ & $2.5 \mu \mathrm{l}$ & $125 \mu 1$ & $245 \mu 1$ \\
\hline & $\begin{array}{l}\text { Pooled STA Master } \\
\text { Mix }\end{array}$ & $0.5 \mu 1$ & $25 \mu 1$ & $49 \mu 1$ \\
\hline & $\begin{array}{ll}\text { DNA } & \text { Suspension } \\
\text { Buffer } & \end{array}$ & $0.75 \mu \mathrm{l}$ & $37.5 \mu \mathrm{l}$ & $73.5 \mu 1$ \\
\hline \multicolumn{2}{|l|}{ cDNA } & 1.25 & & \\
\hline \multicolumn{2}{|c|}{ Total Volume } & $5 \mu \mathrm{l}$ & & \\
\hline
\end{tabular}

$3.75 \mu \mathrm{l}$ of the Pre-mix was aliquoted onto a 96 well PCR plate. To each well $1.25 \mu \mathrm{cDNA}$ was added, making a total volume of $5 \mu 1$. The plate was vortexed and centrifuged.

\subsubsection{Thermal Cycling}

The plate was placed in the thermal cycler for the following PCR program.

\section{Condition}

\begin{tabular}{lcc}
\hline \hline Hold & $95^{\circ} \mathrm{C}$ & $2 \mathrm{~min}$ \\
\hline Denaturation* & $95^{\circ} \mathrm{C}$ & $15 \mathrm{sec}$ \\
\hline Primer annealing/ elongation* & $60{ }^{\circ} \mathrm{C}$ & $4 \mathrm{~min}$ \\
\hline Hold & $4{ }^{\circ} \mathrm{C}$ & \\
\hline
\end{tabular}

Temperature

Time

*in 14 cycles 


\subsubsection{Exonuclease I Treatment}

To remove unincorporated primers after preamplification of each template, an exonuclease I step was performed.

\begin{tabular}{|l|c|c|c|}
\hline \multicolumn{1}{|c|}{ Component } & $\begin{array}{c}\text { Volume/ } \\
\text { reaction tube } \\
(\boldsymbol{\mu l})\end{array}$ & $\begin{array}{c}\text { Volume for 48 } \\
\text { reactions + } \\
\text { overage }(\boldsymbol{\mu l})\end{array}$ & $\begin{array}{c}\text { Volume for 96 } \\
\text { reactions + } \\
\text { overage ( } \boldsymbol{\mu l})\end{array}$ \\
\hline Water & $1.4 \mu \mathrm{l}$ & $70 \mu \mathrm{l}$ & $137.2 \mu \mathrm{l}$ \\
\hline Exonuclease I Reaction Buffer & $0.2 \mu \mathrm{l}$ & $10 \mu \mathrm{l}$ & $19.6 \mu \mathrm{l}$ \\
\hline Exonuclease I (20 U/ $\mu \mathrm{l})$ & $0.4 \mu \mathrm{l}$ & $20 \mu \mathrm{l}$ & $39.2 \mu \mathrm{l}$ \\
\hline Total Volume & $\mathbf{2 \mu \mathrm { l }}$ & & \\
\hline
\end{tabular}

To each well on the PCR plate, $2 \mu$ of the above master mix was added to the cDNA, making a final volume of $7 \mu$ l. The PCR plate was then vortexed and centrifuged and placed in the thermo cycler, as listed below.

\begin{tabular}{llc} 
Condition & Temperature & Time \\
\hline \hline Digest & & $30 \mathrm{~min}$ \\
\hline Inactivate & $37^{\circ} \mathrm{C}$ & $15 \mathrm{~min}$ \\
\hline Hold & $80^{\circ} \mathrm{C}$ & \\
\hline
\end{tabular}

The final products were diluted 5-fold by adding $18 \mu \mathrm{l}$ DNA suspension buffer to the $7 \mu 1$ of cDNA, making a total cDNA volume of $25 \mu$. The cDNA was stored at $-20{ }^{\circ} \mathrm{C}$ till further use. 
Preparing Sample Pre-Mix and Samples for Gene Expression using Fluidigm Dynamic Arrays

\begin{tabular}{|c|l|c|c|}
\hline \multicolumn{2}{|c|}{ Component } & Volume per inlet $(\boldsymbol{\mu l})$ & $\begin{array}{c}\text { Volume per inlet } \\
\text { with overage }(\boldsymbol{\mu l})\end{array}$ \\
\hline Pre-mix & $\begin{array}{l}\text { 2X SsoFast EvaGreen } \\
\text { Supermix with Low ROX }\end{array}$ & $2.5 \mu \mathrm{l}$ & $3 \mu \mathrm{l}$ \\
\hline $\begin{array}{l}\text { 20X DNA Binding Dye } \\
\text { Sample Loading Reagent }\end{array}$ & $0.25 \mu \mathrm{l}$ & $0.3 \mu \mathrm{l}$ \\
\hline PreAmp and Exo I- treated sample & $2.25 \mu \mathrm{l}$ & $2.7 \mu \mathrm{l}$ \\
\hline Total Volume & & $\mathbf{5} \boldsymbol{\mu l}$ & \\
\hline
\end{tabular}

$3.3 \mu 1$ of the Pre-xix for each sample was aliquoted and mixed with $2.7 \mu 1$ of the PreAmp and Exo I treated cDNA on a 96 well plate. The plate was centrifuged and kept ready to be loaded onto the chip.

\section{Preparing the Assay Mix}

\begin{tabular}{|c|c|c|c|}
\hline \multicolumn{2}{|r|}{ Component } & Volume per inlet $(\mu \mathrm{l})$ & Volume per inlet \\
\hline \multirow[b]{2}{*}{ Assay mix } & 2X Assay Loading Reagent & $2.5 \mu \mathrm{l}$ & $3 \mu 1$ \\
\hline & $\begin{array}{l}1 \mathrm{X} \text { DNA Suspension } \\
\text { Buffer }\end{array}$ & $2.05 \mu 1$ & $2.46 \mu 1$ \\
\hline \multicolumn{2}{|c|}{$100 \mu \mathrm{M}$ each primer pair } & $0.45 \mu 1$ & $0.54 \mu \mathrm{l}$ \\
\hline \multicolumn{2}{|c|}{ Total Volume } & $5 \mu \mathrm{l}$ & \\
\hline
\end{tabular}

$5.46 \mu \mathrm{l}$ of the Assay Mix was aliquoted and mixed with $0.54 \mu \mathrm{l}$ of each primer pair mix $(100 \mu \mathrm{M})$, to make a total volume of $5 \mu$. The plate was centrifuged and kept ready to be loaded onto the chip. 


\subsubsection{Priming and Loading the Dynamic Array IFC}

To prime the Dynamic Array chip prior to use, a control line fluid was injected into each accumulator on opposite sides of the chip. The blue protective film was removed from the bottom of the chip, and the chip was placed in an integrated fluidic circuit (IFC) Controller (MX for the 48.48 Dynamic Array or the HX for the 96.96 Dynamic Array) to be primed for the experiment. Following priming, $5 \mu$ of each assay was loaded onto the left side of the chip, and $5 \mu 1$ of each sample was loaded into the respective inlets on the right side of the chip. The Array was then returned to the respective IFC Controller to mix the samples and assays onto the chip. The chip was then run using the BioMark Gene Expression Data Collection software, following the given parameters for the 96.96 dynamic arrays.

\begin{tabular}{lcccc} 
Condition & $\begin{array}{c}\text { Temperature } \\
\left({ }^{\circ} \mathrm{C}\right)\end{array}$ & $\begin{array}{c}\text { Time } \\
(\mathbf{s})\end{array}$ & $\begin{array}{c}\text { Biomark HD } \\
\text { Ramp Rate } \\
\left({ }^{\circ} \mathrm{C} / \mathrm{s}\right)\end{array}$ & $\begin{array}{c}\text { Biomark } \\
\text { Ramp Rate } \\
\left({ }^{\circ} \mathrm{C} / \mathrm{s}\right)\end{array}$ \\
\hline \hline Thermal Mix & 70 & 2400 & 5.5 & 2 \\
& 60 & 30 & 5.5 & 2 \\
\hline Hot Start & 95 & 60 & 5.5 & 2 \\
\hline PCR* & 96 & 5 & 5.5 & 2 \\
\hline Melting Curve & 60 & 20 & 5.5 & 1 \\
& 60 & 3 & 1 & 1 \\
\hline
\end{tabular}

* 40 cycles

\subsubsection{Histology}

\subsubsection{Tissue cross-sections}

The small and large intestinal biopsies were fixed in $10 \%$ formalin overnight at $4{ }^{\circ} \mathrm{C}$ and subsequently dehydrated in ethanol solutions of concentrations $50 \%, 70 \%, 80 \%, 96 \%$ and 100 $\%$, for 75 min each. This was followed by immersion in xylol for $2 \times 75$ min each. The biopsies were then immersed in liquid paraffin wax $\left(56-58^{\circ} \mathrm{C}\right)$ and then biopsies were embedded in paraffin blocks. Sections of $5 \mu \mathrm{m}$ were cut from pre-chilled blocks using a microtome, and transferred to glass slides, and dried overnight at $37^{\circ} \mathrm{C}$ and stored at room temperature. 


\subsubsection{Hematoxylin and eosin staining}

Paraffin sections were stained with Hematoxylin and eosin (HE). The Hematoxylin dye stains acidic or basophilic structures, such as DNA and RNA to a purplish blue color, whereas Eosin dye stains acidophilic or basic structures such as cytoplasmic proteins, a red or pink color. The slides with the tissue sections were deparaffinated with xylol $(3 \times 7 \mathrm{~min})$, and subsequently rehydrated with ethanol from $100 \%, 96 \%, 75 \%$ and $60 \%$ for 5 min each and washed with $\mathrm{dH}_{2} \mathrm{O}$ for 5 min. The tissues sections were then stained with Mayer's hemalaum solution for 5 mins, rinsed for 10 min and stained with eosin for $5 \mathrm{~min}$. The slides were washed again and dehydrated with an increasing ethanol concentration, $60 \%, 75 \%, 96 \%$ and $100 \%$, followed by isopropanol and xylol for 5 min each. The tissues were embedded with Roti-Histokitt.

\subsubsection{Immunohistochemistry}

The paraffin sections were stained with specific antibodies to detect proteins of interest. The sections were boiled in citrate buffer $(3 \times 5 \mathrm{~min} ; 10 \mathrm{mM}, \mathrm{pH}$ 6.0). On cooling, the tissues were blocked with IHC blocking solution (4\% BSA in PBS) for $1 \mathrm{hr}$ at room temperature (RT). The primary antibody was diluted in PBS with $1 \%$ BSA and incubated overnight at $4{ }^{\circ} \mathrm{C}$. Following this, the tissue sections were incubated in $3 \% \mathrm{H}_{2} \mathrm{O}_{2}(30 \%)$ in PBS at RT for 10 min to block endogenous peroxidases, and washed with PBS ( $3 \times 5 \mathrm{~min})$. Incubation with secondary biotinconjugated antibody for $1 \mathrm{hr}$ at RT, followed by another round of washing ( $3 \times 5 \mathrm{~min}$ ) with PBS. The samples were incubated with HRP-streptavidin for $1 \mathrm{hr}$ at RT and stained with DAB substrate solution ( $0.05 \%$ DAB, $0.015 \% \mathrm{H}_{2} \mathrm{O}_{2}$, dissolved in PBS). The slides were than counterstained with hematoxylin for $3 \mathrm{~min}$, and rinsed in $\mathrm{H}_{2} \mathrm{O}$ for $5 \mathrm{~min}$, dehydrated and mounted with Roti-Histokitt for imaging. 


\subsubsection{Statistical Analysis}

\subsubsection{Animal gene expression data analysis}

The $\mathrm{dCt}$ values were calculated as $\mathrm{dCt}$ is the $\mathrm{Ct}_{\text {Geomean(housekeeping genes) }-} \mathrm{Ct}_{\text {(genes of interest), where Geomean }}$ is the geometrical mean of the housekeeping genes Gapdh and Hprt. The gene expression data for mice and rats was tested for normality using the Shapiro-Wilk normality test. The data was not normally distributed, therefore the p-values were calculated for the $\mathrm{dCt}$ values using the MannWhitney U-test on Prism Graphpad or SPSS. The data was corrected for multiple comparisons using Benjamin Hochberg's false discover rate (FDR), and plotted as graphs using Prism Graphpad.

\subsubsection{Human gene expression data analysis}

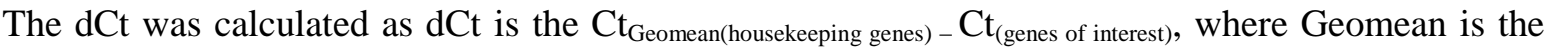
geometrical mean of the housekeeping genes, GAPDH and HPRT. The $\mathrm{dCt}$ values were transformed using Blom's rank-based normalization on SPSS.

\subsubsection{EBMT Risk Score}

The EBMT risk score was calculated according to Gratwohl et al. (Gratwohl, 2012). The risk score is independent of the underlying disease. It comprises of the summation of different clinical factors that affect GvHD. The different factors are depicted in the Table 2.12. The score ranges from 0 to 7, with 7 being the worst score. For example, male patients, above the age of 40 (score $=2$ ), undergoing HSCT from an unrelated female donor (score $=\mathbf{1}$ ), after over a year of diagnosis of primary disease ( score $=\mathbf{2}$ ) at a late stage ( risk of post-transplant complications and mortality. 


\begin{tabular}{|c|c|}
\hline Risk factor & Score points \\
\hline \multicolumn{2}{|l|}{ Patient age (years) } \\
\hline$<20$ & 0 \\
\hline $20-40$ & 1 \\
\hline$>40$ & 2 \\
\hline \multicolumn{2}{|l|}{ Stage of disease* } \\
\hline Early & 0 \\
\hline Intermediate & 1 \\
\hline Late & 2 \\
\hline \multicolumn{2}{|c|}{$\begin{array}{l}\text { Time interval from diagnosis to transplant } \\
\text { (months)** }\end{array}$} \\
\hline$<12$ & 0 \\
\hline$>12$ & 1 \\
\hline \multicolumn{2}{|l|}{ Donor Type**** } \\
\hline HLA-identical sibling donor & 0 \\
\hline Unrelated donor, other & 1 \\
\hline $\begin{array}{l}\text { Donor recipient sex } \\
\text { combination }\end{array}$ & \\
\hline All other & 0 \\
\hline Female donor, male recipient & 1 \\
\hline
\end{tabular}

\subsubsection{Selection of covariates}

We modeled our multivariate analysis to compensate for several factors that affect the HSCT outcome, such as the EBMT score, age of donor, type of conditioning, source of stem cells. Based on the selected covariates, the gene expression patterns of the different outcomes such as aGvHD and TRM were determined.

\subsubsection{Step-wise regression model}

A step-wise regression model using $\mathrm{R}$ software (http://www.R- project.org) was generated to compensate for all the important covariates. The following covariates were selected for the final model: EBMT score, source of stem cell, type of conditioning regimen, blood group, steroid treatment, CMV status of the donor-recipient pairs, age of donor and time from transplant to date of biopsy taken. Based on the model, we assessed the change in gene expression for the selected variables. Two sample t-tests were performed and $\mathrm{p}$-values and $\mathrm{t}$ values have been listed in the 
respective tables. Significant p-values $(<0.05)$ and p-values that were near significance $(<0.15)$ have been shown. The t-value represents the size of the difference relative to variation observed in the sample data, and positive (+) t-values depict upregulation and negative (-) t-values show downregulation.

\subsubsection{Hierarchical cluster heatmaps}

Hierarchical clustering of heatmaps was performed using R statistical packages. The hierarchical cluster was performed using 'hclust' on scaled data using 'matrix' and heatmap was generated using 'ggplot2' packages. 


\section{Results}

\subsection{Animal aGvHD models}

\subsection{1 aGvHD mouse model}

We studied the regulation of gene expression between aGvHD mice $(n=17)$, where irradiated BALB/c mice were transplanted with BM and T cells from C57BL/6 mice, BM control mice $(n=6)$, where irradiated BALB/c mice were transplanted with BM from C57BL/6 mice and compared the gene regulation with healthy control mice $(n=15)$. The different in clinical scores between the transplant mouse groups is depicted in Figure 3.1. The aGvHD mice model is fully MHC- mismatched, and the aGvHD mice, BM control and healthy control mice are of the same strain $(\mathrm{BALB} / \mathrm{c})$. We compared the gene expression between aGvHD mice and healthy controls to determine the overall gene regulation during aGvHD in the different target tissues (*red). To determine the effect of preconditioning and BM transplantation on the gene regulation, we compared the gene expression between BM control mice and healthy controls (*green). Finally, to isolate the genes that were differentially regulated as a result of aGvHD alone, we compared the gene expression between the aGvHD and $\mathrm{BM}$ control mice (*blue).

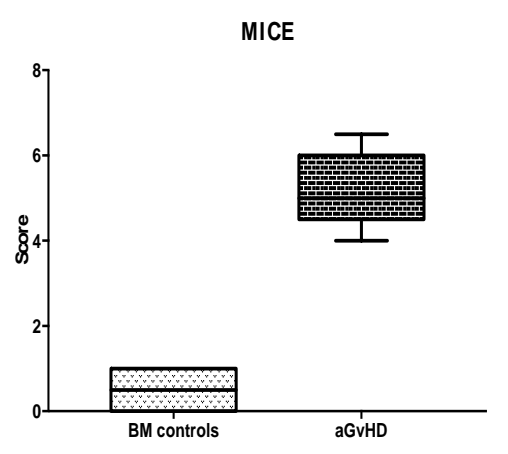

Figure 3.1: Clinical aGvHD score in mouse.

The aGvHD mice had scores of $5.5 \pm 0.41$ and the BM controls had scores of 1 on day 6 . The difference in scores was showing the extent of disease in the aGvHD mice in comparison with the BM controls $(\mathrm{p}<0.001)$.

\subsection{2 aGvHD rat model}

In rats, the gene expression was studied between aGvHD rats $(n=6)$, where PVG. $1 \mathrm{~N}$ rats were irradiated and transplanted with BM and T cells from PVG.7B rats and control rats $(n=6)$ where PVG rats were irradiated and transplanted with BM and T cells from PVG.7B rats. In the rat aGvHD model, we used MHC congenic rat strains of the same genetic background (PVG) that only differ in their MHC region. The rats were sacrificed between 3-4 weeks post transplant. 


\subsubsection{Gene expression profiling in different aGvHD tissues}

The fold-change of the gene expression and the respective p-values have been specified for the genes that were significantly regulated, as (Fold change; p-value). In the supplement, the table with the entire gene expression results is given (Section 7). All the graphs have been plotted with the relative gene expression $(\mathrm{dCt})$ on the $\mathrm{y}$-axis and target organs on the $\mathrm{x}$-axis.

We have studied the regulation patterns of several selected genes in different mouse aGvHD tissues, rat aGvHD tissues and compared these expression patterns to human GI biopsies in patients that have undergone HSCT. Several genes encoding inflammatory cytokines and chemokines have been studied in different aGvHD tissues. We wanted to validate the expression of several previously known genes and compare their regulation patterns in the different tissues simultaneously and in the different species. Furthermore, we also validated the regulation patterns of previously identified candidate genes that were found to be regulated in rat and human skin biopsies, in the different aGvHD tissues in the different species. In addition, based on our results for the MICA-129 polymorphism, we also studied the regulation patterns of the activating NK receptor, KLRK1 (NKG2D) and its ligands, as well as another important activating NK receptor, CD226 (DNAM-1) and its ligands in the different aGvHD tissues in the two animal models and compared their expression patterns to human GI biopsies from HSCT patients.

\subsection{Important genes regulated in different mouse tissues due to preconditioning and aGvHD}

Several genes that are expected to be regulated during aGvHD were selected for our study. In addition, we included other genes that are important in adaptive immune responses that we expected to be important during aGvHD. Here we show the gene expression data in different mouse tissues as a result of preconditioning and aGvHD for genes associated with Th1, Th2, Th17 and Treg responses. In addition, several genes important during aGvHD immune responses were also studied.

In this section, results show the regulation of genes as a result of overall aGvHD between mouse tissues affected by aGvHD and healthy controls, as well as the effect of preconditioning on the regulation patterns of these genes in the different mouse tissues. Moreover, we also show the alterations in gene expression patterns between aGvHD affected mouse tissues and BM control mice that have been irradiated and transplanted with BM alone. This model allows us to determine genes that are regulated solely due to aGvHD occurrence and not as a result of preconditioning or the transplantation procedure. 


\subsubsection{Expression of cytokines associated with Th1 responses in mice}

The most important cytokines involved in the inflammatory response during aGvHD are IL-2 and IFN- $\gamma$, and are produced by Th1 cells. IFN- $\gamma$ and IL- 12 drive the differentiation of Th1 cells. Th1 cells are known to be the initial drivers of aGvHD (Ferrara et al., 2009). Here we studied the gene expression of Ifng, Il12a, Il2 and Tnf.

Except in the liver, the overall mRNA expression of Ifng was increased in mice with aGvHD compared to health controls, in the lung (33.76; $\mathrm{p}=0.008)$, skin $\left(\mathbf{3} \times \mathbf{1 0}^{\mathbf{6}} ; \mathrm{p}=0.008\right)$, spleen (5.63; $\mathrm{p}=0.008)$, small intestine (1.16 x 10 $\left.\mathbf{8}^{\mathbf{3}} ; \mathrm{p}=0.008\right)$, and large intestine (43.6; $\left.\mathrm{p}=0.008\right)$. In the lung and spleen, the expression of Ifng was increased primarily as a result of preconditioning in the BM control mice in comparison with healthy controls (6.25; $p=0.004$ and 7.23; $p=0.004)$, and further increased as a result of aGvHD in the lung $(\mathbf{5 . 4 1} ; \mathrm{p}=0.008)$, small intestine $(\mathbf{2 3 5 . 1 8} ; \mathrm{p}=0.008)$ and large intestine (95.52; $\mathrm{p}=0.008)$ respectively, compared to BM control mice (Figure 3.2A).

The expression of $I l 12 a$ was downregulated in the spleen alone, due to preconditioning $(\mathbf{0 . 1} ; \mathrm{p}=$ 0.004) and in aGvHD mice compared to healthy controls ( $\mathbf{0 . 1 1} ; \mathrm{p}=0.008)$ (Figure 3.2B). Il2, on the other hand, showed a mixed expression pattern, whereby it was significantly reduced in the spleen of aGvHD mice compared to BM control mice and compared to healthy controls $(\mathbf{0 . 6 5} ; \mathrm{p}=$ 0.004), and in the liver of aGvHD mice compared to BM controls $(\mathbf{0 . 0 3} ; \mathrm{p}=0.004)$, and significantly increased in the lung as a result of preconditioning in BM control mice compared to healthy controls $(3.19 ; p=0.04)$ and further upregulated in the lung $(5.4 ; p=0.004)$ and large intestine (3.31; $\mathrm{p}=0.004)$ of aGvHD mice compared to healthy controls (Figure 3.2C). The proinflammatory cytokine Tnf was significantly reduced in the spleen during preconditioning (0.34; $\mathrm{p}=0.004)$ and further reduced in aGvHD mice compared to BM controls $(\mathbf{0 . 4 1} ; \mathrm{p}=0.004)$ as well as healthy controls $(\mathbf{0 . 1 4} ; \mathrm{p}=0.008)$. In the lung and large intestine, the expression of Tnf was strongly upregulated in aGvHD mice compared to BM control mice (2.93; $p=0.004$ and 5.63; $\mathrm{p}=0.004)$, and compared to healthy control mice respectively $(\mathbf{2 . 0 6} ; \mathrm{p}=0.004$ and 3.15; $\mathrm{p}=$ 0.004) (Figure 3.2D).

Overall, the observed increase in the expression of Ifng and Tnf is consistent with severe inflammation in the different target tissues and is most predominant in the intestine. 


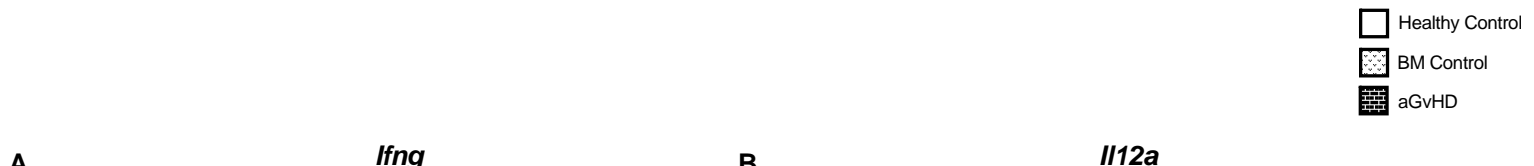

A

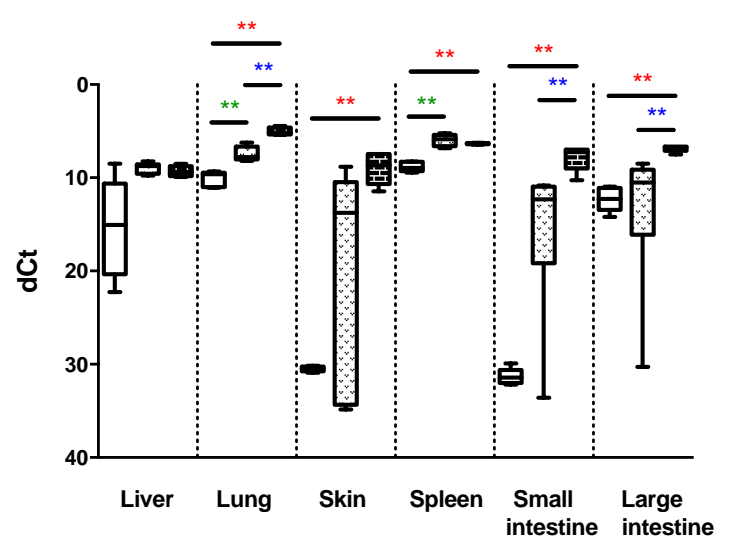

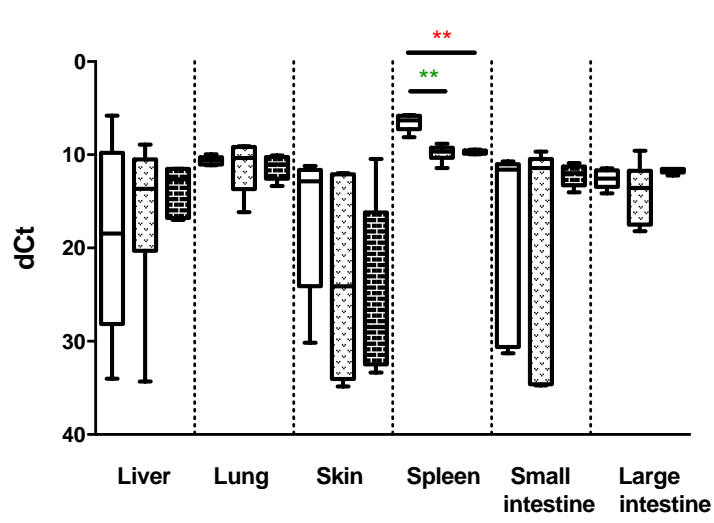

C

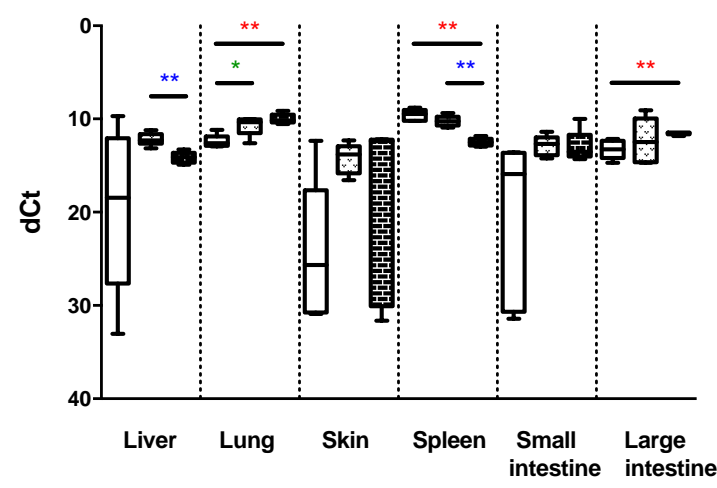

D

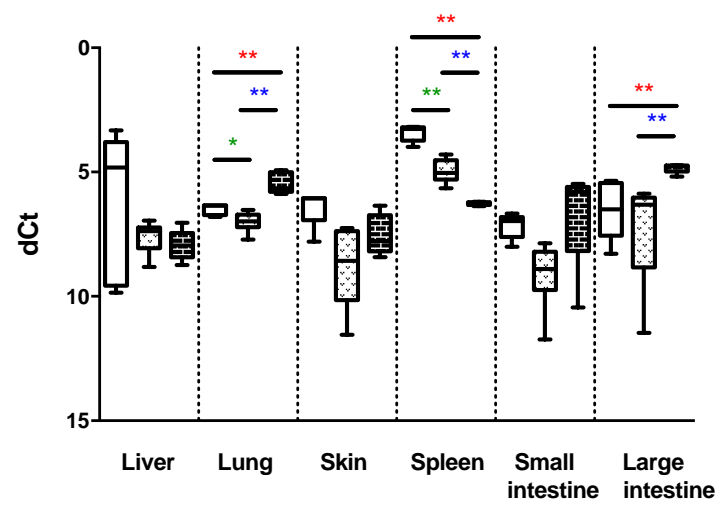

Figure 3.2: mRNA expression of cytokines associated with Th1 responses in different tissues in mice.

Relative mRNA expression of cytokines associated with Th1 responses, in aGvHD mice $(\mathrm{n}=17)$ in comparison with normal healthy controls $(n=15)($ red $)$, in aGvHD mice compared to BM control mice $(n=6)$ (blue) and preconditioning alone by comparing BM control mice to healthy controls (green). The y-axis shows relative expression $(\mathrm{dCt})$, and $\mathrm{x}$ axis shows different mice groups in each tissue type. The $\mathrm{p}$-values were calculated by Mann-Whitney U-test, and were corrected for FDR by Benjamin Hochberg correction. $* \mathrm{P}<0.05, * * \mathrm{P}<0.01, * * * \mathrm{P}<0.001, * * * * \mathrm{P}<0.0001$ (significantly regulated genes in aGvHD mice compared to healthy controls)

$* \mathrm{P}<0.05, * * \mathrm{P}<0.01, * * * \mathrm{P}<0.001, * * * * \mathrm{P}<0.0001$ (significantly regulated genes in aGvHD mice compared to $\mathrm{BM}$ controls)

$* \mathrm{P}<0.05, * * \mathrm{P}<0.01, * * * \mathrm{P}<0.001, * * * * \mathrm{P}<0.0001$ (significantly regulated genes in $\mathrm{BM}$ control mice due to preconditioning) 


\subsubsection{Expression of cytokines associated with Th2 responses in mice}

Th2 cells are characterized by the production of cytokines IL-4, IL-5, IL-13 and IL-33. IL-13 requires IL-4R $\alpha$ for regulating Th2 responses (Barner et al., 1998). We observed an overall increased regulation of the Th2 cytokines in the different tissues in mice. Il4 was significantly increased in the aGvHD mice compared to healthy controls in the liver (133.21; $p=0.02)$, skin (59.98; $\mathrm{p}=0.007)$, spleen (2.2 $\left.\times \mathbf{1 0}^{2} ; \mathrm{p}=0.004\right)$, small (2.84; $\left.\mathrm{p}=0.029\right)$ and large intestines (5.48; $\mathrm{p}=0.001)$. The expression of $I l 4$ was significantly increased in the liver also as a result of preconditioning in the BM control mice compared to the healthy controls $(\mathbf{5 6 . 9 2} ; \mathrm{p}=0.001)$, and in the large intestine between aGvHD mice and BM control mice $(\mathbf{2 . 7 6} ; \mathrm{p}=0.008)$ (Figure 3.3A).

The expression of Il5 was also significantly increased in the large intestine in aGvHD mice compared to healthy controls $(\mathbf{6 8 4 . 1 9} ; \mathrm{p}=0.008)$, but was significantly downregulated in the lung $(\mathbf{0 . 2 9} ; \mathrm{p}=0.016)$ (Figure 3.3B). Ill3 mRNA was significantly upregulated in the lung (1.52; $\mathrm{p}=$ $0.016)$ and large intestine $(\mathbf{6 . 8 5} ; \mathrm{p}=0.008)$ in aGvHD mice compared to healthy controls (Figure 3.3C). Il4ra was significantly increased in the lung (19.28; $p=0.019)$, small $(\mathbf{3 . 2 3} ; \mathrm{p}=0.001)$ and large intestine (4.91; $\mathrm{p}=0.001)$ in aGvHD mice compared to healthy controls. In the lung (27.39; $\mathrm{p}=0.001)$ and large intestine $(\mathbf{2 . 5 4} ; \mathrm{p}=0.007)$, the increase in the expression of Il4ra was also in part due to preconditioning, and in the small intestine, Il4ra was strongly increased in the aGvHD mice in comparison with the BM controls (2.18; $\mathrm{p}=0.008$ ) (Figure 3.3D). Similarly, Il33 was also strongly upregulated in aGvHD mice in the skin $(\mathbf{3 . 4 4} ; \mathrm{p}=0.007)$ and large intestine $(\mathbf{4 . 7 8} ; \mathrm{p}=$ 0.007), compared to healthy controls and in the small intestine (3.55; $\mathrm{p}=0.008)$ compared to BM control mice (Figure 3.3E). IL-33 is highly expressed on Th2 cells and serves as a ligand for the IL-33R (IL-1RL1 or ST2) (Yagami et al., 2010) and has been shown previously to worsen experimental GvHD via its interaction with its receptor IL-1RL1 on alloreactive cells (Reichenbach et al., 2015).

Taken together, we observed a strong increase in the expression of Th2 cytokines, mainly between aGvHD mice and healthy controls. The Th2 response was also most significant in the intestine. Preconditioning alone did not significantly alter the expression of these cytokines, suggesting that they are mainly regulated as a result of the aGvHD disease. 


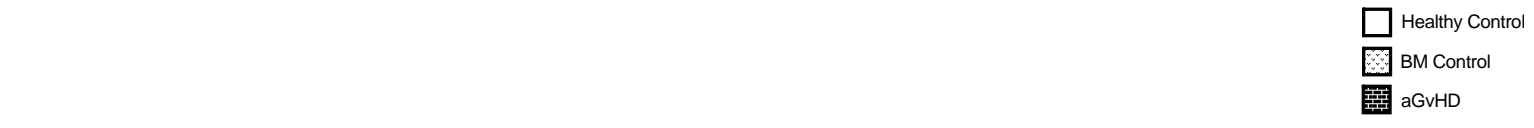

A

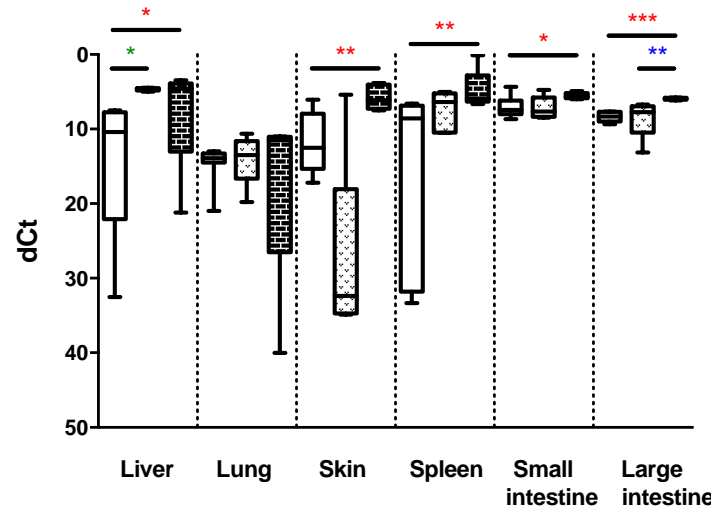

C

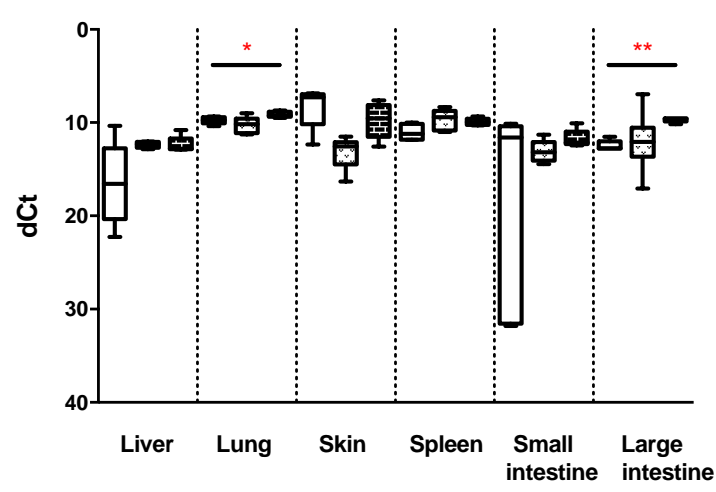

$\mathbf{E}$

II33

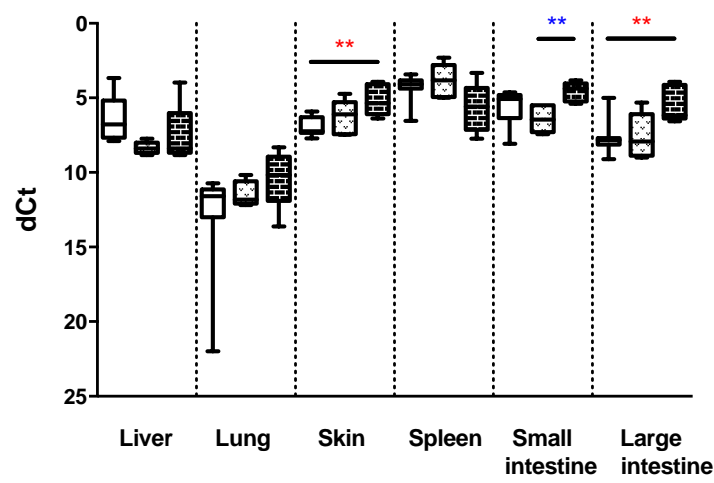

B

II5

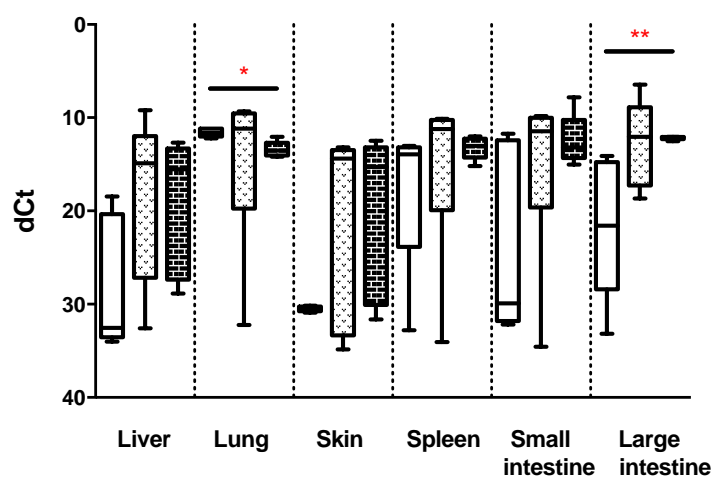

D

II4ra

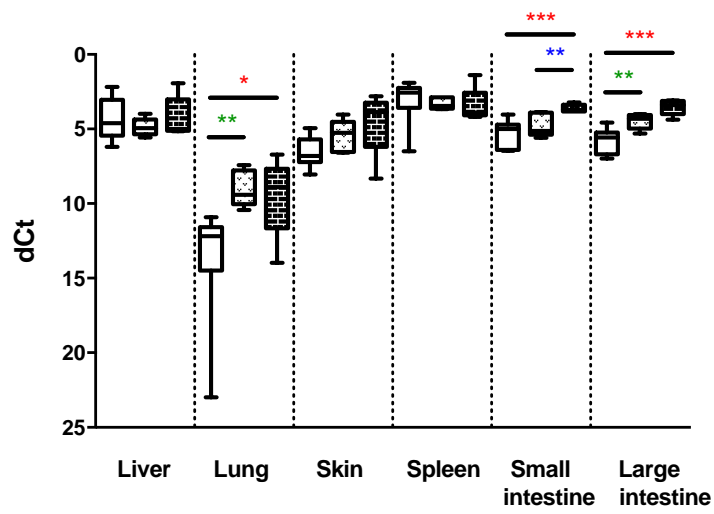

Figure 3.3: mRNA expression of cytokines associated with Th2 responses in different tissues in mice.

Relative mRNA expression of cytokines associated with Th2 responses, in aGvHD mice $(n=17)$ in comparison with normal healthy controls $(n=15)($ red $)$, in aGvHD mice compared to BM control mice $(n=6)$ (blue) and preconditioning alone by comparing BM control mice to healthy controls (green). The y-axis shows relative expression $(\mathrm{dCt})$, and $\mathrm{x}$ axis shows different mice groups in each tissue type. The $\mathrm{p}$-values 
were calculated by Mann-Whitney U-test, and were corrected for FDR by Benjamin Hochberg correction. $* \mathrm{P}<0.05, * * \mathrm{P}<0.01, * * * \mathrm{P}<0.001, * * * * \mathrm{P}<0.0001$ (significantly regulated genes in aGvHD mice compared to healthy controls)

$* \mathrm{P}<0.05, * * \mathrm{P}<0.01, * * * \mathrm{P}<0.001, * * * * \mathrm{P}<0.0001$ (significantly regulated genes in aGvHD mice compared to BM controls)

$* \mathrm{P}<0.05, * * \mathrm{P}<0.01, * * * \mathrm{P}<0.001, * * * * \mathrm{P}<0.0001$ (significantly regulated genes in $\mathrm{BM}$ control mice due to preconditioning)

\subsubsection{Expression of cytokines associated with Th17 responses in mice}

Th17 cells require TGF- $\beta$ and IL-6 to differentiate (Bettelli et al., 2006), and they are characterized by the production of IL-17A, IL-21 and IL-22 (Korn et al., 2009; Park et al., 2005; Weaver et al., 2007). IL-23 is required for the stabilization of Th17 cells, along with TNF- $\alpha$ and IL-1 $\beta$ (Aggarwal et al., 2002). In mice, cutaneous aGvHD is suggested to be predominantly driven by Th17 responses (Carlson et al., 2009). An augmented damage to the lung and skin tissue has been associated with Th17 cells (Yi et al., 2009). Moreover, it was suggested that Th17 cells were sufficient but not necessary to induce aGvHD (Iclozan et al., 2010). To study the regulation of Th17 responses in our study, here we show the regulation of the Th17 cytokine genes, Il6, Ill7a, Il22 and Il23a.

As expected, we observed a significant increase in the expression of these cytokines in the different tissues during aGvHD. The expression of $1117 a$ was significantly increased in the spleen and large intestine in aGvHD mice compared to healthy controls $(17.88 ; p=0.032$ and 14.56; $p=0.008)$ respectively (Figure 3.4A). The expression of $I l 6$ was significantly increased in the lung $(1.41 ; \mathrm{p}=$ 0.03), spleen (5.43; $\mathrm{p}=0.004)$ and large intestine $\left(\mathbf{1 . 2} \times \mathbf{1 0}^{2} ; \mathrm{p}=0.004\right)$ as a result of preconditioning in BM control mice compared to healthy controls. The gene expression was further augmented in the aGvHD mice compared to healthy control mice in the lung $(4.57 ; p=0.008)$, small (791.68; $\mathrm{p}=0.008)$ and large intestines $\left(3.2 \times \mathbf{1 0}^{2} ; \mathrm{p}=0.008\right)$. The $I l 6$ expression was also significantly increased in the aGvHD mice compared to the BM control mice in the lung $(\mathbf{3 . 2 5} ; \mathrm{p}=$ 0.004) and small intestine $(\mathbf{3 . 3 7} ; \mathrm{p}=0.004)$ (Figure 3.4B). Similarly, Il22 was significantly increased in the lung due to preconditioning in $\mathrm{BM}$ control mice $\left(\mathbf{1 . 0 4} \times \mathbf{1 0}^{4} ; \mathrm{p}=0.004\right)$, and was further increased in aGvHD mice compared to healthy controls in the lung $\left(6.4 \times 10^{4} ; p=0.008\right)$ and large intestine $\left(\mathbf{1 . 4} \times \mathbf{1 0}^{2} ; \mathrm{p}=0.008\right)$ (Figure 3.4C). Il23a was significantly increased in the aGvHD large intestine compared to BM control mice $\left(3.8 \times \mathbf{1 0}^{2} ; \mathrm{p}=0.004\right)$ as well as healthy controls $\left(4.9 \times 10^{3} ; p=0.008\right)$, but reduced in the lung as a result of preconditioning $\left(0.7 \times 10^{-3} ; p\right.$ $=0.009)$ (Figure 3.4D). 
The Th17 response was also strongest in the intestine, followed by the lung. Th17 cytokines are mainly associated with cutaneous aGvHD in mice, however in this study, these cytokines were not differentially regulated in the skin. Th17 genes, in addition to Th1 and Th2 responses were significantly upregulated.

A

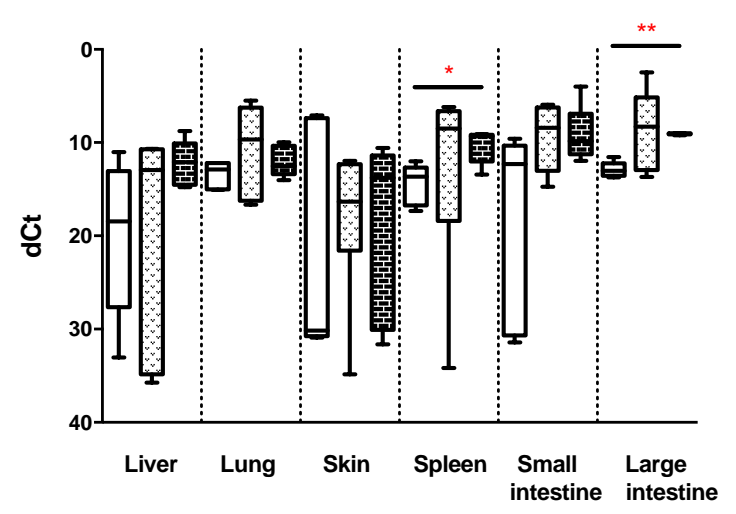

B

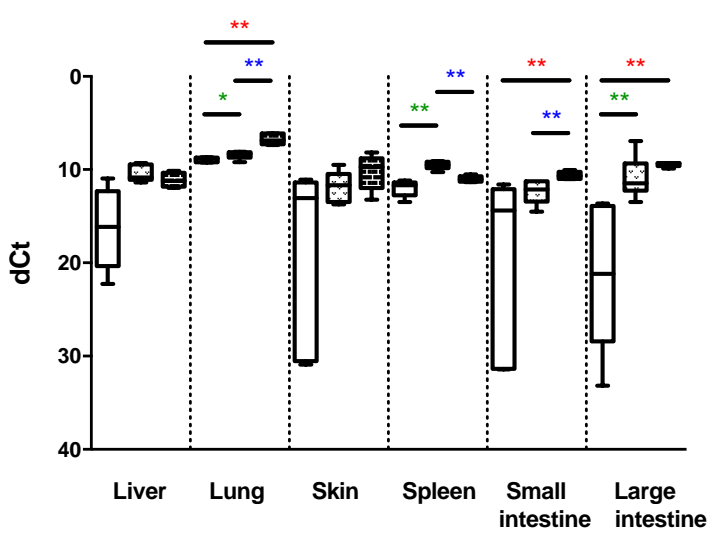

D
C

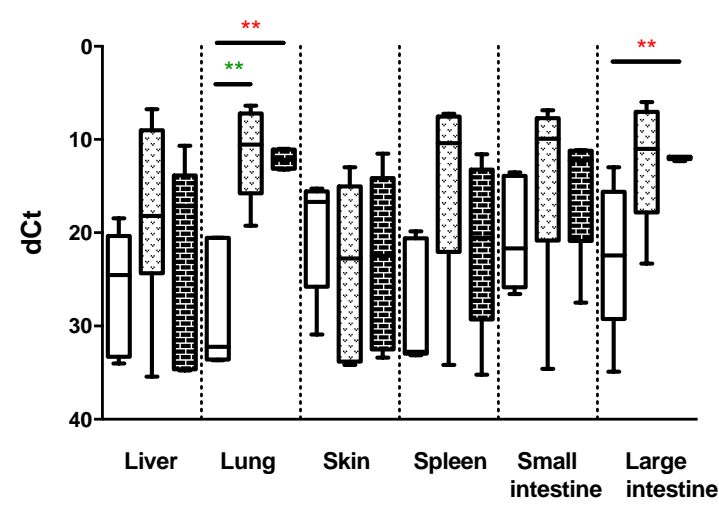

1122

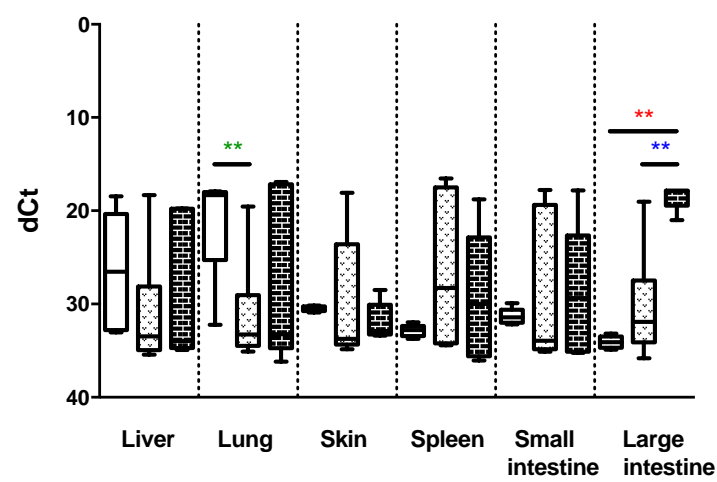

Figure 3.4: mRNA expression of cytokines associated with Th17 responses in different tissues in mice.

Relative mRNA expression of cytokines associated with Th2 responses, in aGvHD mice $(n=17)$ in comparison with normal healthy controls $(\mathrm{n}=15)(\mathrm{red})$, in aGvHD mice compared to BM control mice $(\mathrm{n}=6)$ (blue) and preconditioning alone by comparing BM control mice to healthy controls (green). The y-axis shows relative expression $(\mathrm{dCt})$, and $\mathrm{x}$ axis shows different mice groups in each tissue type. The $\mathrm{p}$-values were calculated by Mann-Whitney U-test, and were corrected for FDR by Benjamin Hochberg correction. $* \mathrm{P}<0.05, * * \mathrm{P}<0.01, * * * \mathrm{P}<0.001, * * * * \mathrm{P}<0.0001$ (significantly regulated genes in aGvHD mice compared to healthy controls)

$* \mathrm{P}<0.05, * * \mathrm{P}<0.01, * * * \mathrm{P}<0.001, * * * * \mathrm{P}<0.0001$ (significantly regulated genes in aGvHD mice compared to BM controls)

$* \mathrm{P}<0.05, * * \mathrm{P}<0.01, * * * \mathrm{P}<0.001, * * * * \mathrm{P}<0.0001$ (significantly regulated genes in $\mathrm{BM}$ control mice due to preconditioning) 


\subsubsection{Expression of genes associated with Tregs in mice}

Another important subset of T cells, are the Tregs. Here, we observed an increased gene expression pattern for genes that have previously been associated with the expression, regulation or function of Tregs.

The expression of Foxp3 and Il2ra (CD25) is characteristic of nTregs. The gene expression of Foxp3, which is a transcription factor that is critical for the development and function of Tregs, was significantly increased in aGvHD mice compared to the BM controls $(\mathbf{8 . 8 9} ; \mathrm{p}=0.0079)$ in the large intestine (Figure 3.5A). On the other hand, Il2 $\mathrm{ra}$ was significantly increased in almost all the tissues of aGvHD mice compared to BM control mice, in the liver (4.07; $p=0.004)$, lung (4.33; $p$ $=0.004)$, skin (10.12; $p=0.009)$, small $(\mathbf{8 . 7 7} ; \mathrm{p}=0.004)$ and large intestines $(\mathbf{1 0 . 0 8} ; \mathrm{p}=0.004)$. Moreover, in the lung and spleen, Il2 $\mathrm{ra}$ was significantly increased as a result of preconditioning in BM control mice $(1.73 ; p=0.017$ and $1.01 ; p=0.004)$ and in aGvHD mice compared to healthy controls in the lung (7.49; $\mathrm{p}=0.008)$ and large intestine $(\mathbf{1 0 . 2 1} ; \mathrm{p}=0.008)$, however the Il2ra expression was decreased in the spleen $(\mathbf{0 . 6 9} ; \mathrm{p}=0.008$ ) (Figure 3.5B). Il2 $r a$ is expressed not only by Tregs, but on activated $\mathrm{T}$ cells too, suggesting there is an infiltration of activated alloreactive $\mathrm{T}$ cells as well as Tregs in the different target tissues. IL-10 is a Th2 cytokine, but is also produced by Tregs, and both IL-10 and TGF- $\beta$ are important in Treg regulation to reduce immunity and autoimmunity (Carrier et al., 2007; Erhardt et al., 2007). The overall expression of $I l 10$ was not regulated in the tissues, except in the large intestine, where $1 l 10$ was strongly upregulated in aGvHD mice compared to healthy controls (63.27; $\mathrm{p}=0.008$ ) (Figure 3.5C). Tgfbl and Tgfbrl mRNA were both strongly increased in the lung due to preconditioning (7.42; $\mathrm{p}=0.002$ and 12.06; $\mathrm{p}=0.012$ ) (Figure 3.5D and 3.5E). While the Tgfbrl expression was not altered in any other tissues with aGvHD, Tgfbl mRNA was significantly increased in the liver (1.76; $\mathrm{p}=0.022)$, $\operatorname{skin}(\mathbf{2 . 1 5} ; \mathrm{p}$ $=0.012)$ and large intestine $(\mathbf{2 . 0 1} ; \mathrm{p}=0.004)$ in mice with aGvHD compared to healthy controls, and in aGvHD small and large intestines compared to BM control mice (1.97; $p=0.008$ and 2.03; $\mathrm{p}=0.008)$. In contrast, $T g f b 2$ was mostly downregulated due to preconditioning in BM control mice compared to healthy controls in the liver $(\mathbf{0 . 3 7} ; \mathrm{p}=0.001)$, spleen $(\mathbf{0 . 4 1} ; \mathrm{p}=0.001)$ and large intestine $(\mathbf{0 . 3 3} ; \mathrm{p}=0.001)$. However, the mRNA expression of $T g f b 2$ increased significantly in aGvHD large intestine compared to BM controls $(\mathbf{1 . 9 7} ; \mathrm{p}=0.008)$ (Figure 3.5F). 
A

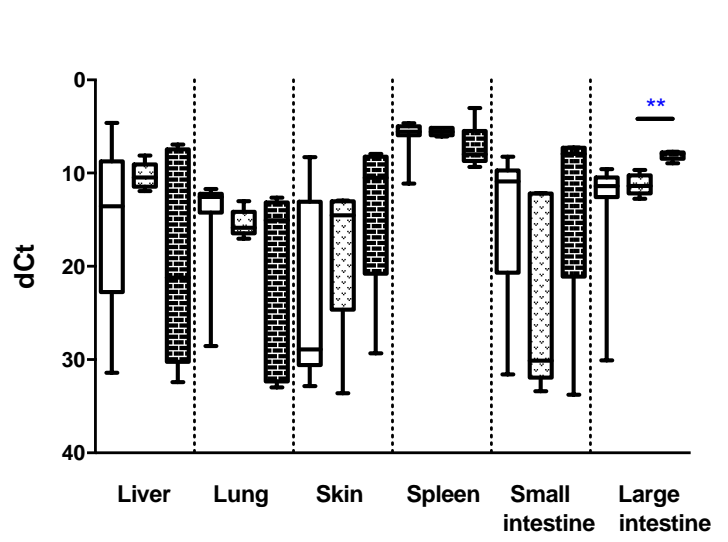

B

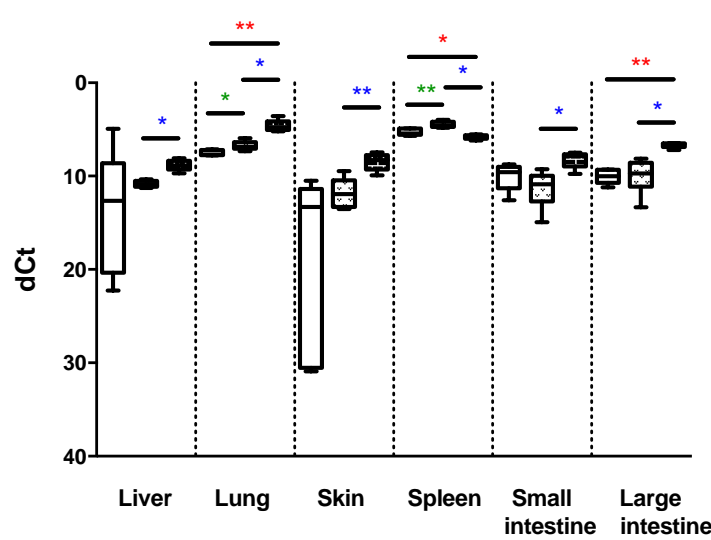

C

II10

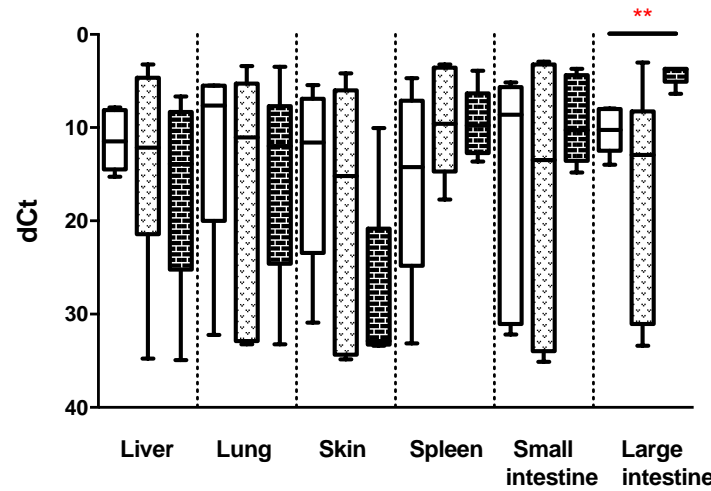

E

Tgfbr1

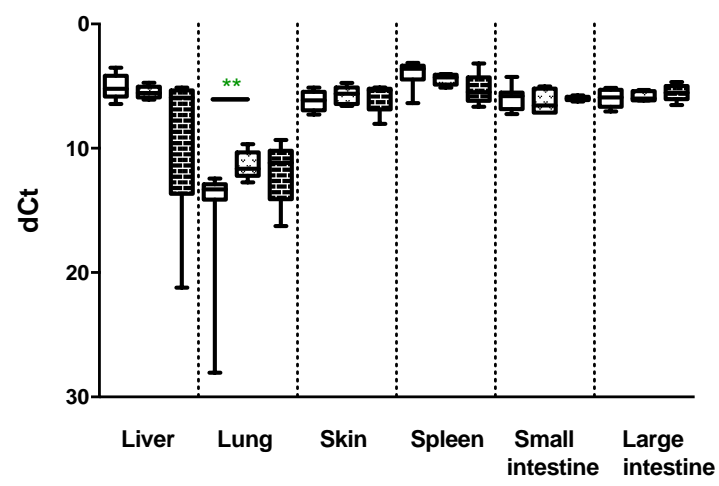

D

Tgfb1

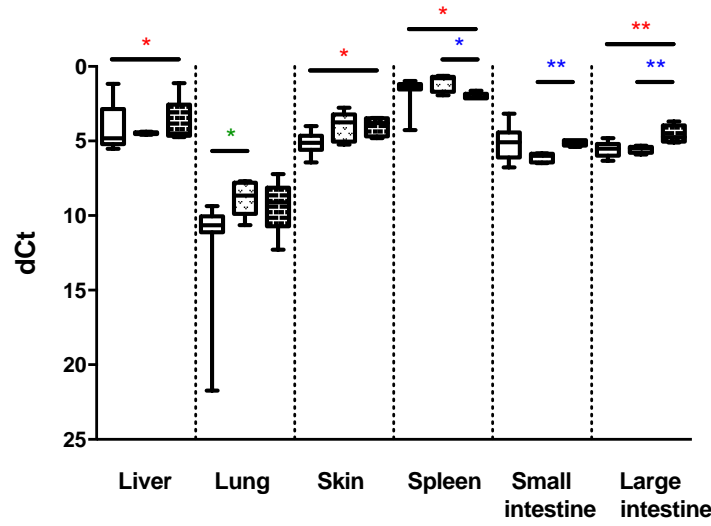

F

Tgfb2

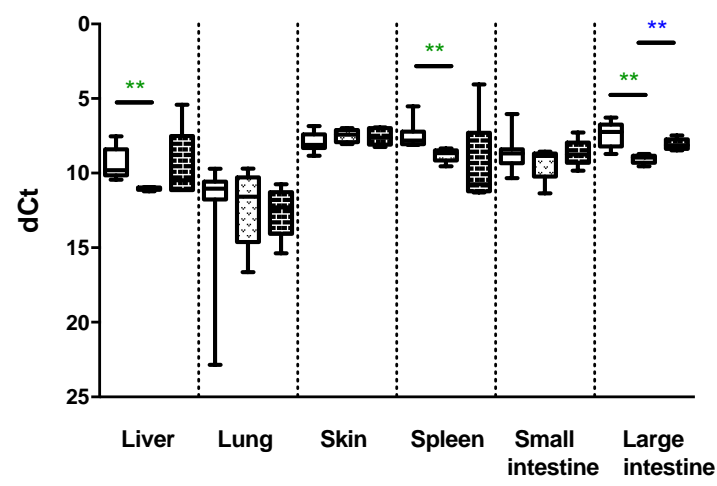


G

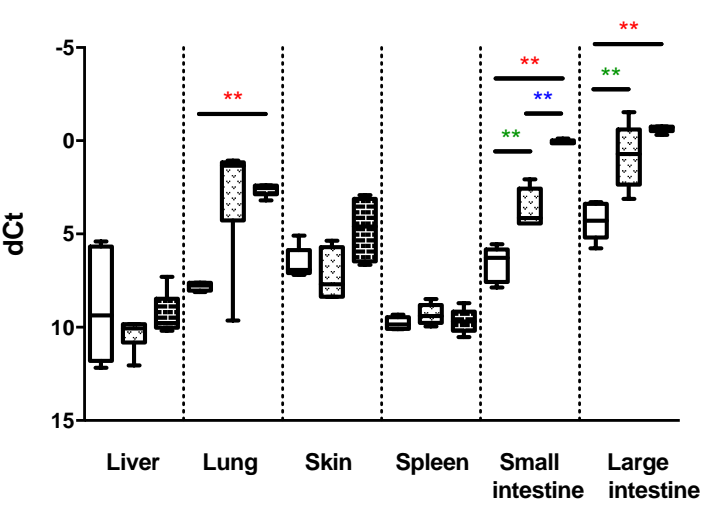

I

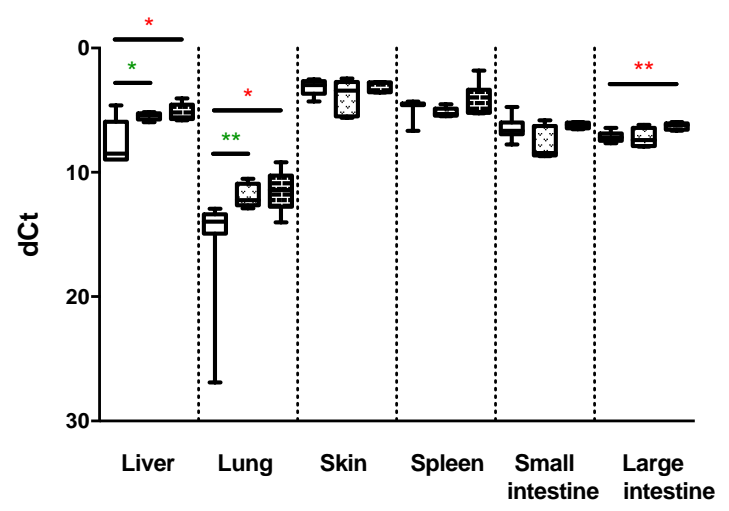

H

Arg1

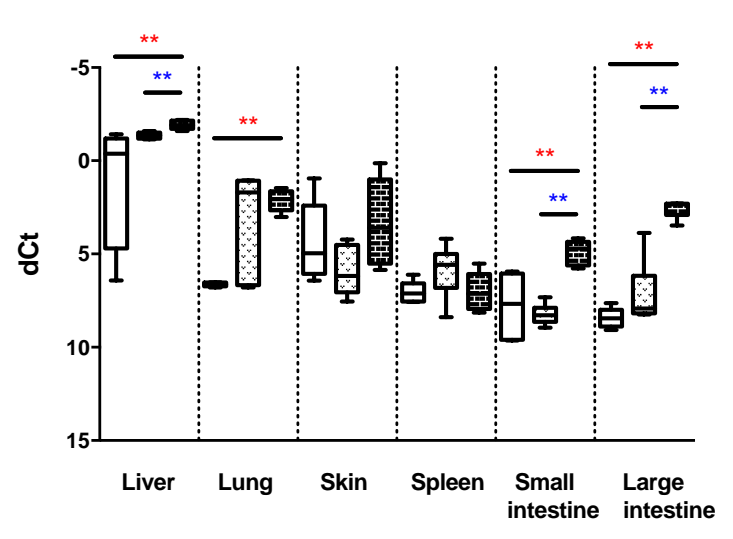

$\mathbf{J}$

Lgals3

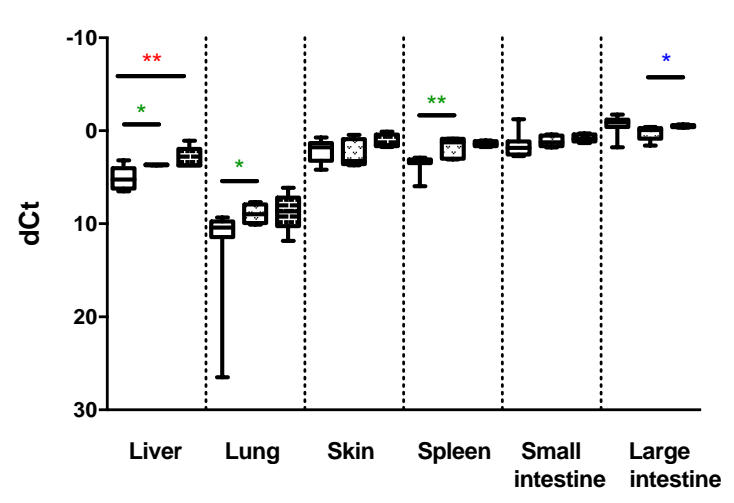

Figure 3.5: mRNA expression of cytokines associated with Tregs responses in different tissues in mice.

Relative mRNA expression of cytokines associated with Tregs responses, in aGvHD mice $(\mathrm{n}=17)$ in comparison with normal healthy controls $(n=15)$ (red), in aGvHD mice compared to BM control mice $(n=6)$ (blue) and preconditioning alone by comparing BM control mice to healthy controls (green). The y-axis shows relative expression $(\mathrm{dCt})$, and $\mathrm{x}$ axis shows different mice groups in each tissue type. The $\mathrm{p}$-values were calculated by Mann-Whitney U-test, and were corrected for FDR by Benjamin Hochberg correction. $* \mathrm{P}<0.05, * * \mathrm{P}<0.01, * * * \mathrm{P}<0.001, * * * * \mathrm{P}<0.0001$ (significantly regulated genes in aGvHD mice compared to healthy controls)

$* \mathrm{P}<0.05, * * \mathrm{P}<0.01, * * * \mathrm{P}<0.001, * * * * \mathrm{P}<0.0001$ (significantly regulated genes in aGvHD mice compared to BM controls)

$* \mathrm{P}<0.05, * * \mathrm{P}<0.01, * * * \mathrm{P}<0.001, * * * * \mathrm{P}<0.0001$ (significantly regulated genes in $\mathrm{BM}$ control mice due to preconditioning)

Similarly, we observed an upregulation of Ido mRNA. IDO1 is strongly linked to the differentiation of Tregs and contributes to their suppressive capacity. The overall expression of Idol was upregulated in aGvHD lung (36.57; $\mathrm{p}=0.008)$, small (93.18; $\mathrm{p}=0.008)$ and large intestine (30.82; $\mathrm{p}=0.008)$ compared to healthy controls. The expression of Idol was increased in both small and large intestines as a result of preconditioning in BM control mice compared to healthy controls $(\mathbf{7 . 7 5} ; \mathrm{p}=0.004$ and 11.19; $\mathrm{p}=0.004)$, and further increased in the small intestine as a result of aGvHD alone in BM controls compared to healthy controls $(\mathbf{1 2 . 0 3} ; \mathrm{p}=0.004)$ (Figure 
3.5G). In addition to IDO, another metabolic enzyme induced by inflammation is Arginase-1 (ARG1) (Rodriguez et al., 2004), that is important in Treg function. Activation of ARG1, reduces arginine, which in turn reduces T cell responses (Highfill et al., 2010; Rodriguez et al., 2004). We observed a significant upregulation in the expression of Argl in the aGvHD liver (9.5; $\mathrm{p}=0.008)$, lung (22.69; $p=0.008)$, small (7.29; $p=0.008)$ and large intestine (59.31; $p=0.008)$ compared to healthy controls and in the liver $(\mathbf{1 . 4 8} ; \mathrm{p}=0.009)$, small $(\mathbf{9 . 9 2} ; \mathrm{p}=0.004)$ and large $(\mathbf{2 4 . 6 4} ; \mathrm{p}=$ 0.004) intestines compared to BM control (Figure 3.5H). The Interleukin-1 receptor-like 2 precursor (IL-1RL2) has been shown to be expressed on human Tregs (Pfoertner et al. 2006). The expression of Illrl2 was significantly increased in the liver $(\mathbf{6 . 0 3} ; \mathrm{p}=0.019)$ and the lung $(\mathbf{1 1 . 6 2} ; \mathrm{p}$ $=0.001)$ as a result of preconditioning in the BM control mice compared to healthy controls. This expression was further augmented due to aGvHD in the mice compared to healthy controls in the liver (7.22; $p=0.012)$ and lung (15.08; $p=0.019)$ respectively (Figure 3.5I). Galacten-3 (LGALS3) was also reported to be expressed on human Treg subsets (Pfoertner et al. 2006). An important role of LGALS3 is limiting TCR mobility causing a restricted TCR-mediated signaling on $\mathrm{T}$ cells, subsequently resulting in a change of the cytokine profile of $\mathrm{T}$ cells, thereby regulating the effector cells and homeostasis of immune cells (Demetriou et al., 2001). The expression of Lglas3 was significantly increased in the BM control liver (4.87; $p=0.019)$, lung $(9.3 ; p=0.019)$ and spleen $(3.29 ; \mathrm{p}=0.007)$ compared to healthy controls due to preconditioning, and it was further increased in aGvHD liver compared to healthy control liver (7.63; $p=0.007)$, and in aGvHD large intestine $(\mathbf{1 . 6 3} ; \mathrm{p}=0.016)$, compared to BM control mice (Figure 3.5J).

Tregs are generally present in the peripheral lymphoid organs in the presence of TGF- $\beta$, which is consistent with our findings in the large intestine where we observed an increased expression of all the genes associated with Tregs except $T g f b 2$, suggesting an increase in Treg population in the different tissues, but mainly the intestine.

\subsubsection{Expression of IFN- $\gamma$ inducible chemokine receptor $\operatorname{Cxcr} 3$ and its ligands Cxcl9, Cxcl10 and Cxcl11 in mice}

In general, we observed an increased expression pattern of the IFN- $\gamma$ inducible Th1 chemokines Cxcl9, Cxcl10, Cxcl11 and their receptor Cxcr3. However, these chemokines showed a mixed expression pattern in the liver, compared to the other tissues.

The mRNA expression of $C x c r 3$ was significantly upregulated in the aGvHD mice compared to healthy controls in the lung (21.16; 0.008), skin $\left(1.9 \times 10^{6} ; 0.008\right)$, small $(\mathbf{1 2 . 5 9} ; 0.008)$ and large (4.4 $\left.\times 10^{10} ; 0.008\right)$ intestines, whereas it was significantly reduced in the liver $(\mathbf{0 . 4 1} ; 0.008)$. Our results show that the regulation of $C x c r 3$ was predominantly a result of preconditioning in the liver 
(0.2; $\mathrm{p}=0.008)$, and due to aGvHD in the lung (49.15; $\mathrm{p}=0.004)$. On the other hand, the expression of $C x c r 3$ was increased in the skin $\left(2.1 \times 10^{4} ; \mathrm{p}=0.004\right)$ and large intestine $\left(\mathbf{1 . 9} \times \mathbf{1 0}^{\mathbf{6}}\right.$; $\mathrm{p}=0.004)$ of the BM control mice compared to healthy controls as a result of preconditioning, and further increased in the aGvHD mice skin (92.4; $\mathrm{p}=0.004)$, small $(\mathbf{5 1 . 3 1} ; \mathrm{p}=0.004)$ and large $(\mathbf{2 . 2}$ $\mathbf{x} \mathbf{1 0}^{4} ; \mathrm{p}=0.004$ ) intestines compared to BM control mice (Figure 3.6A).

Cxcl9 mRNA was significantly increased in the liver (48.67; $\mathrm{p}=0.002)$ and lung $(\mathbf{7 . 8 3} ; \mathrm{p}=0.018)$ as a result of preconditioning in BM control mice compared to healthy controls, and its expression was strongly increased in aGvHD mice compared to healthy controls in the liver $(\mathbf{1 0 . 5 4} ; \mathrm{p}=$ 0.007), lung (9.07; $\mathrm{p}=0.01)$, small $(\mathbf{3 . 1 8} ; \mathrm{p}=0.001)$ and large $(\mathbf{1 0 . 5 4} ; \mathrm{p}=0.007)$ intestines (Figure 3.6B). Similarly, Cxcl10 mRNA was significantly increased in the BM control lung (18.44; $\mathrm{p}=$ 0.004) due to preconditioning further increased in aGvHD lung (47.87; $\mathrm{p}=0.008)$ compared to healthy controls, and in aGvHD skin (21.77; $\mathrm{p}=0.008)$, small (10.46; $\mathrm{p}=0.008)$ and large (21.32; $\mathrm{p}=0.008)$ intestines compared to healthy controls. However, in the liver and spleen, the expression of $\mathrm{Cxcl10}$ was increased as a result of preconditioning (13.08; $\mathrm{p}=0.004$ and 8.03; $\mathrm{p}=0.004)$ in the $\mathrm{BM}$ control mice compared to healthy controls and reduced significantly in aGvHD mice $(\mathbf{0 . 1 7} ; \mathrm{p}$ $=0.004$ and $\mathbf{0 . 1 2} ; \mathrm{p}=0.004$ ) compared to BM controls, respectively (Figure 3.6C). Cxcl11 mRNA showed an increased expression in the BM control lung $(19.99 ; \mathrm{p}=0.004)$ due to preconditioning and aGvHD lung $(\mathbf{6 5 . 7 1} ; \mathrm{p}=0.008)$ compared to healthy control mice. The expression of Cxcl11 was also significantly increased in the aGvHD large intestine compared to healthy controls (64.82; $\mathrm{p}=0.008)$ as well as BM controls (31.09; $\mathrm{p}=0.004)$ (Figure 3.6D).

Overall, the expression of $\mathrm{Cxcr} 3$ and its ligands $\mathrm{Cxcl}$, $\mathrm{Cxcl} 10$ and $\mathrm{Cxcl11}$ might have contributed to aGvHD in mice. 

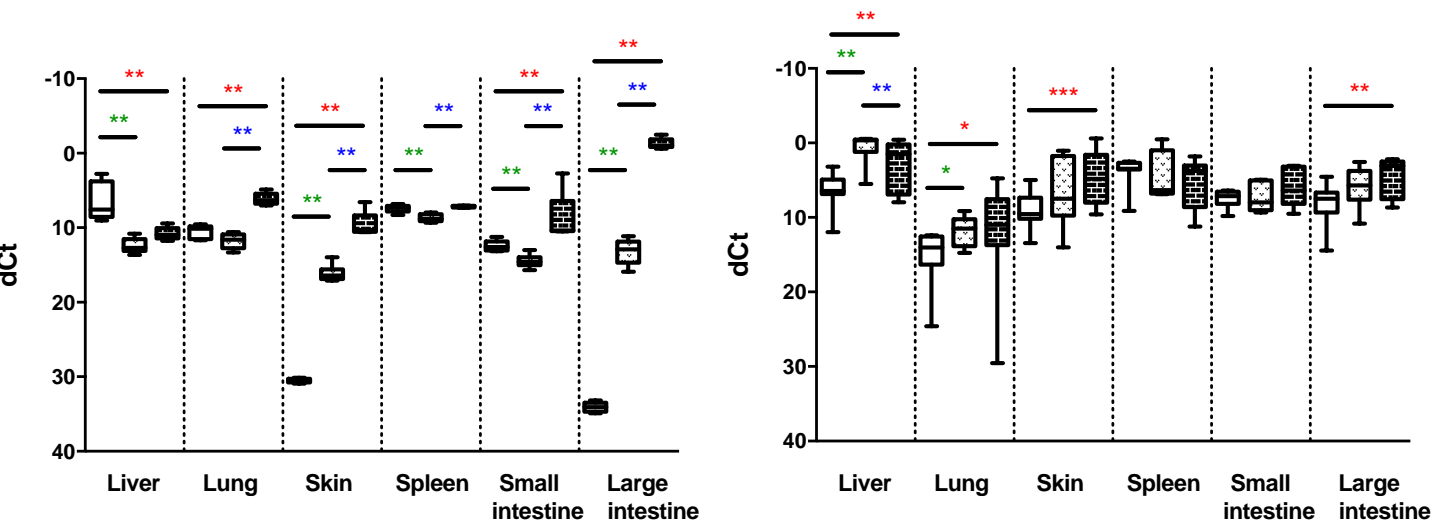

C

\section{Cxcl10}

(IP-10)

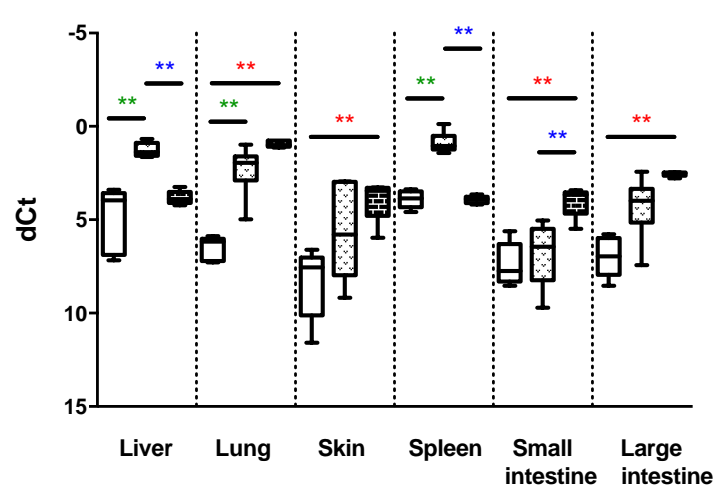

D

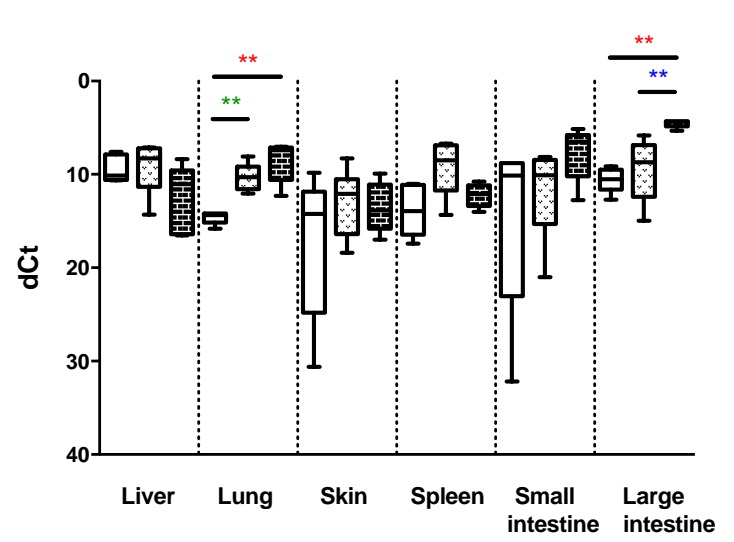

Figure 3.6: mRNA expression of chemokines induced by IFN- $\gamma$ in different tissues in mice. Relative mRNA expression of chemokine receptor A. Cxcr3 and its ligands B. Cxcl9 (Mig), C. Cxcl10 (IP$10)$ and D. Cxcl11 (IP-9), in aGvHD mice $(\mathrm{n}=17)$ in comparison with normal healthy controls $(\mathrm{n}=15)$ (red), in aGvHD mice compared to BM control mice $(\mathrm{n}=6)$ (blue) and preconditioning alone by comparing BM control mice to healthy controls (green). The $\mathrm{y}$-axis shows relative expression $(\mathrm{dCt})$, and $\mathrm{x}$ axis shows different mice groups in each tissue type. The p-values were calculated by Mann-Whitney U-test, and were corrected for FDR by Benjamin Hochberg correction.

$* \mathrm{P}<0.05, * * \mathrm{P}<0.01, * * * \mathrm{P}<0.001, * * * * \mathrm{P}<0.0001$ (significantly regulated genes in aGvHD mice compared to healthy controls)

$* \mathrm{P}<0.05, * * \mathrm{P}<0.01,{ }^{*} * \mathrm{P}<0.001, * * * * \mathrm{P}<0.0001$ (significantly regulated genes in aGvHD mice compared to BM controls)

$* \mathrm{P}<0.05, * * \mathrm{P}<0.01, * * * \mathrm{P}<0.001, * * * * \mathrm{P}<0.0001$ (significantly regulated genes in $\mathrm{BM}$ control mice due to preconditioning) 


\subsubsection{Expression of the chemokine receptors Ccr1, Ccr4, Ccr5 and their ligands $\mathrm{Ccl}$, $\mathrm{Ccl5}$ and $\mathrm{Ccl} 4$ in mice}

Here, we studied the mRNA expression of $C c l 9$ and its receptor $C c r 1, C c l 4$ and its receptor $C c r 5$, and $C c l 5$ and its receptor Ccr4. Overall, the gene expression of the chemokine Ccl4 was significantly increased in all the tissues during aGvHD. Ccl4 was significantly increased in the aGvHD mice compared to the BM controls in the liver (3.23; $p=0.004)$, lung (4.09; $p=0.004)$, small (11.97; $p=0.004)$ and large $(\mathbf{1 4 . 4 2} ; \mathrm{p}=0.004)$ intestines, and its expression was further increased in the lung $(\mathbf{8 . 0 2} ; \mathrm{p}=0.004)$, spleen $(\mathbf{2 . 0 5} ; \mathrm{p}=0.008)$, small $(\mathbf{1 6 . 9 3} ; \mathrm{p}=0.008)$ and large (11.87; $\mathrm{p}=0.008)$ intestines in the aGvHD mice compared to healthy controls. Furthermore, Ccl4 was also significantly increased due to preconditioning in the BM control skin $\left(3.3 \times 10^{5} ; \mathrm{p}=\right.$ 0.004) and further increased in aGvHD skin $\left(\mathbf{8 . 5} \times \mathbf{1 0}^{7} ; \mathrm{p}=0.008\right)$ compared to the healthy controls (Figure 3.7A). Ccl5, on the other hand exhibited a more complex expression pattern in the different mice tissues. Overall, the expression of $C c l 5$ was downregulated in the liver $(\mathbf{0 . 2 3} ; \mathrm{p}=0.004)$, lung (0.27; $\mathrm{p}=0.017)$, skin $(\mathbf{0 . 1 2} ; \mathrm{p}=0.009)$, spleen $(\mathbf{0 . 3 1} ; \mathrm{p}=0.004)$ and large $(\mathbf{0 . 0 6} ; \mathrm{p}=0.004)$ intestines in $\mathrm{BM}$ control mice compared to healthy controls as a result of preconditioning. $\mathrm{Ccl} 5$ was further downregulated in the spleen of aGvHD mice compared to BM control mice $(\mathbf{0 . 4 6} ; \mathrm{p}=$ 0.009), as well as healthy controls $(\mathbf{0 . 1 4} ; \mathrm{p}=0.008)$. Additionally, in the large intestine, Ccl5 expression was reduced during aGvHD compared to healthy controls $(\mathbf{0 . 3 7} ; \mathrm{p}=0.008)$. However, the expression of $C c l 5$ was significantly increased in aGvHD mice compared to BM controls in the liver (2.81; $\mathrm{p}=0.004)$, $\operatorname{skin}(\mathbf{8 . 7 8} ; \mathrm{p}=0.009)$ and large intestine $(\mathbf{6 . 2 7} ; \mathrm{p}=0.009)$ (Figure 3.7B).

On the other hand, except for the liver, Ccl9 was significant upregulated in all the tissues during aGvHD. In aGvHD mice, $C c l 9$ was strongly increased in the lung (31.98; $\mathrm{p}=0.019)$, skin (29.18; $\mathrm{p}=0.004)$, spleen $(\mathbf{5 . 9 6} ; \mathrm{p}=0.001)$, small $(\mathbf{3 . 7 8} ; \mathrm{p}=0.001)$ and large $(\mathbf{1 1 . 6 1} ; \mathrm{p}=0.001)$ intestines. Moreover, in the large intestine, a significant upregulation of $C c l 9$ was also observed in the aGvHD mice compared to the BM controls $(\mathbf{9 . 2 6} ; \mathrm{p}=0.008$ ) (Figure 3.7C). Similarly $C c r l$ was significantly increased in the aGvHD liver $(17.84 ; p=0.012)$, skin $(47.37 ; p=0.029)$, spleen (4.64; $\mathrm{p}=0.001)$, small $(4.5 ; \mathrm{p}=0.001)$ and large $(6.62 ; \mathrm{p}=0.002)$ intestines compared to healthy controls, and in the spleen $(\mathbf{2 . 6 4} ; \mathrm{p}=0.008)$, small $(\mathbf{5 . 3 1} ; \mathrm{p}=0.008)$ and large $(\mathbf{2 . 5 7} ; \mathrm{p}=0.016)$ intestines compared to BM control mice (Figure 3.7D).

In contrast, the gene expression of $\mathrm{Ccr} 4$ was significantly reduced in the different tissues during aGvHD. In the lung $(\mathbf{0 . 3 7} ; \mathrm{p}=0.004)$, skin $(\mathbf{0 . 1 9} ; \mathrm{p}=0.004)$ and small intestine $(\mathbf{0 . 3 2} ; \mathrm{p}=0.004)$ Ccr4 is reduced due to preconditioning in BM control mice compared to healthy controls. The regulation is further reduced in the aGvHD lung $(\mathbf{0 . 3 5} ; \mathrm{p}=0.008)$, spleen $(\mathbf{0 . 0 5} ; \mathrm{p}=0.008)$ and small intestine $(\mathbf{0 . 4 1} ; \mathrm{p}=0.008)$ and increased in large intestine $(\mathbf{1 . 5 7} ; \mathrm{p}=0.032)$ compared to 
healthy controls, and in the aGvHD spleen compared to BM controls $(\mathbf{0 . 1 1} ; \mathrm{p}=0.004)$ (Figure 3.7E). The overall expression of $C c r 5$ was significantly increased in the liver (3.01; $\mathrm{p}=$ 0.004), spleen (1.56; $p=0.004)$, small (3.82; $p=0.004)$ and large $(3.15 ; p=0.004)$ intestines in aGvHD mice compared to healthy controls. In addition, Ccr5 is significantly increased in the BM control liver $(\mathbf{2 . 8 1} ; \mathrm{p}=0.001)$ compared to healthy controls due to preconditioning, whereas in the small and large intestines, the expression of $C c r 5$ was significantly increased in the aGvHD mice (7.42; $p=0.008$ and 3.95; $p=0.008$ ) compared to BM controls as well (Figure 3.7F).

A

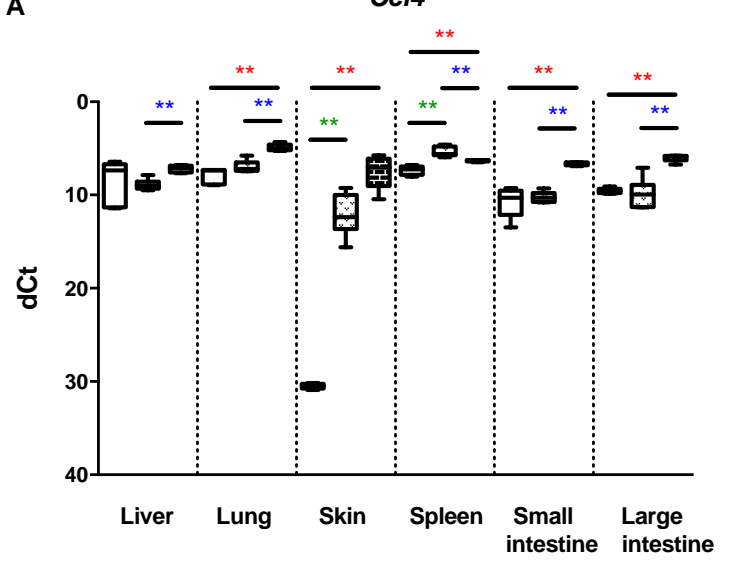

B

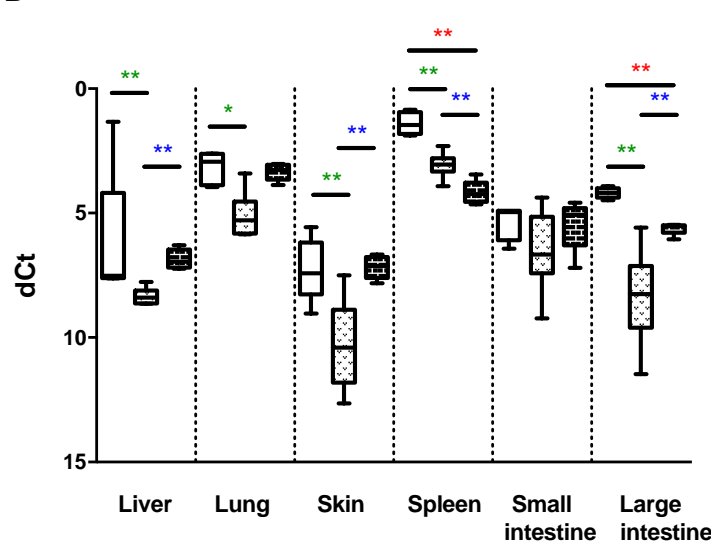

C

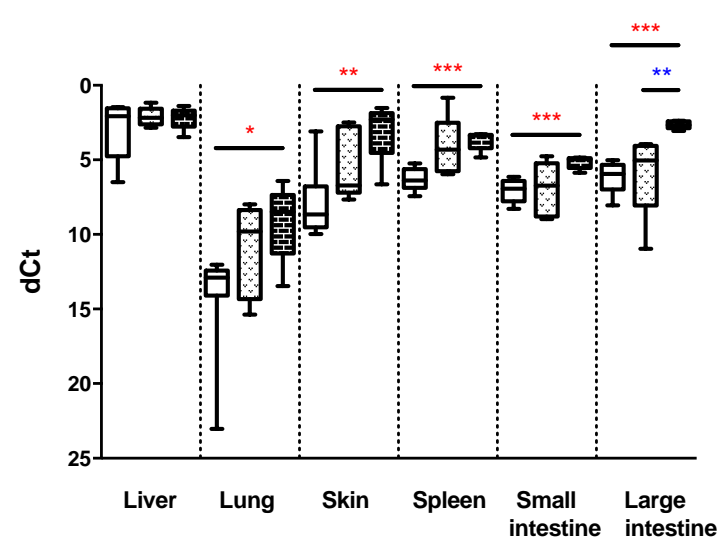

D

Ccr1

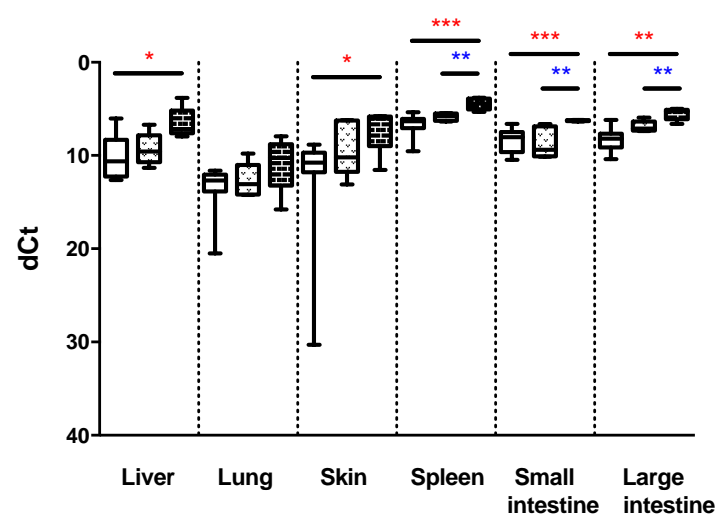


E

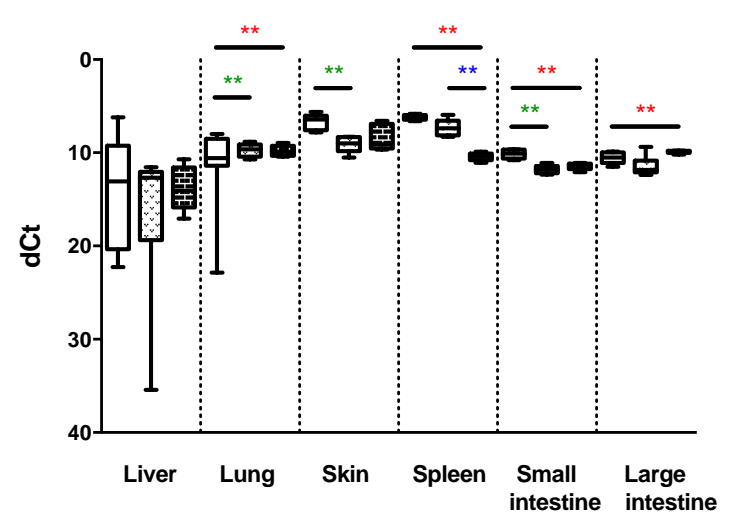

$\mathbf{F}$

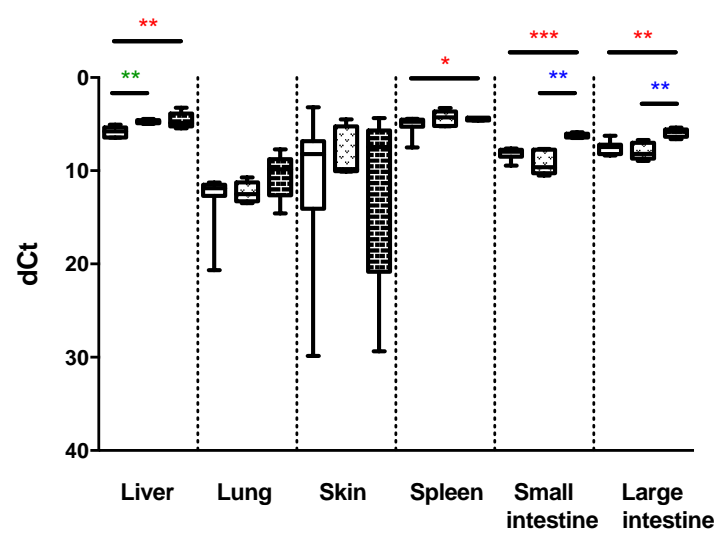

Figure 3.7: mRNA expression of chemokine receptors $C c r 1, C c r 4, C c r 5$ and ligands $C c l 4, C c l 5$ and $\mathrm{Ccl} 9$ in different tissues in mice.

Relative mRNA expression of chemokines A. Ccl4 B. Ccl5 C. Ccl9 and chemokine receptors D. Ccrl E. $C c r 4$ F. $C c r 5$ in aGvHD mice $(\mathrm{n}=17)$ in comparison with normal healthy controls $(\mathrm{n}=15)(\mathrm{red})$, in aGvHD mice compared to BM control mice $(\mathrm{n}=6)$ (blue) and preconditioning alone by comparing BM control mice to healthy controls (green). The $\mathrm{y}$-axis shows relative expression $(\mathrm{dCt})$, and $\mathrm{x}$ axis shows different mice groups in each tissue type. The p-values were calculated by Mann-Whitney U-test, and were corrected for FDR by Benjamin Hochberg correction.

$* \mathrm{P}<0.05, * * \mathrm{P}<0.01, * * * \mathrm{P}<0.001, * * * * \mathrm{P}<0.0001$ (significantly regulated genes in aGvHD mice compared to healthy controls)

$* \mathrm{P}<0.05, * * \mathrm{P}<0.01, * * * \mathrm{P}<0.001, * * * * \mathrm{P}<0.0001$ (significantly regulated genes in aGvHD mice compared to BM controls)

$* \mathrm{P}<0.05, * * \mathrm{P}<0.01, * * * \mathrm{P}<0.001,{ }^{*} * * * \mathrm{P}<0.0001$ (significantly regulated genes in $\mathrm{BM}$ control mice due to preconditioning)

\subsubsection{Expression of $\mathrm{Cxcl15,}$ Cxcl16 and chemokine receptor $\mathrm{Cxcr} 4$ in mice}

In addition we studied the expression profile of the other chemokines, Cxcl15 or Il8, Cxcl16 and the chemokine receptor $C x c r 4$.

Cxcl15 showed a mixed expression across the different aGvHD tissues, although it was mostly downregulated during aGvHD. Cxcl15 mRNA expression was significantly reduced in the liver $(\mathbf{0 . 1 7} ; \mathrm{p}=0.004)$, lung $(\mathbf{0 . 5 1} ; \mathrm{p}=0.004)$, spleen $(\mathbf{0 . 2 6} ; \mathrm{p}=0.004)$ and large intestine $(\mathbf{0 . 4 7} ; \mathrm{p}=$ 0.009) in aGvHD mice compared to BM controls. In the the liver $(\mathbf{0 . 0 7} ; \mathrm{p}=0.008)$, lung $(\mathbf{0 . 3} ; \mathrm{p}=$ $0.008)$ and spleen $(\mathbf{0 . 1 9} ; \mathrm{p}=0.008)$, Cxcl15 was further reduced in aGvHD mice compared to healthy controls. In contrast, Cxcl15 mRNA was significantly increased in the small intestine (2.57; $\mathrm{p}=0.016)$ (Figure 3.8A). On the other hand, Cxcl16 expression was strongly increased in the liver $(\mathbf{2 . 9 7} ; \mathrm{p}=0.007)$, skin $(\mathbf{4 . 5 2} ; \mathrm{p}=0.002)$, small $(\mathbf{2 . 1 7} ; \mathrm{p}=0.001)$ and large $(\mathbf{5 . 6 6} ; \mathrm{p}=$ 0.001 ) intestines of aGvHD mice compared to healthy controls. Furthermore, in the BM control skin, the expression of Cxcl16 was significantly increased due to preconditioning (2.02; $p=0.007)$ in comparison with healthy controls, and further increased in the small $(\mathbf{2 . 0 8} ; \mathrm{p}=0.008)$ and large 
(3.09; $\mathrm{p}=0.008$ ) intestines, in aGvHD mice compared to BM controls (Figure 3.8B). Cxcr4 was strongly downregulated in BM control mice compared to healthy controls due to preconditioning in the lung $(\mathbf{0 . 4} ; \mathrm{p}=0.004)$, small $(\mathbf{0 . 0 5} ; \mathrm{p}=0.004)$ and large $(\mathbf{0 . 1 3} ; \mathrm{p}=0.004)$ intestines and its expression was further reduced in aGvHD mice in the lung $(\mathbf{0 . 2 7} ; \mathrm{p}=0.008)$, small $(\mathbf{0 . 0 7} ; \mathrm{p}=$ $0.008)$ and large $(\mathbf{0 . 2 5} ; \mathrm{p}=0.008)$ intestines compared to healthy controls (Figure 3.8C).

A

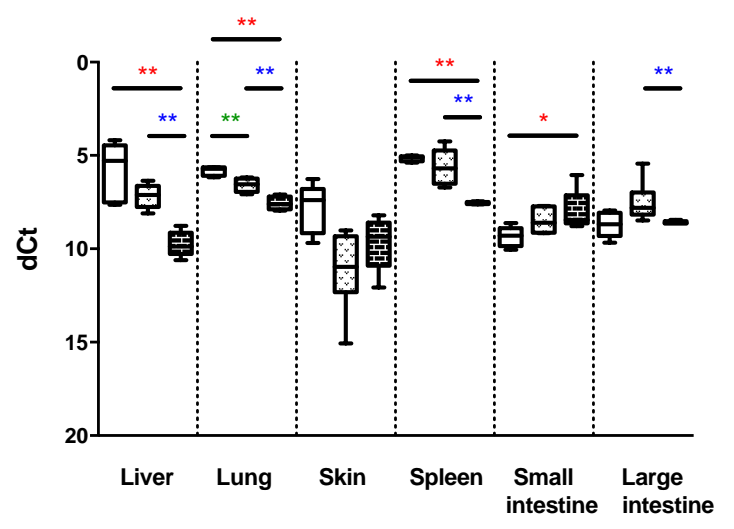

B

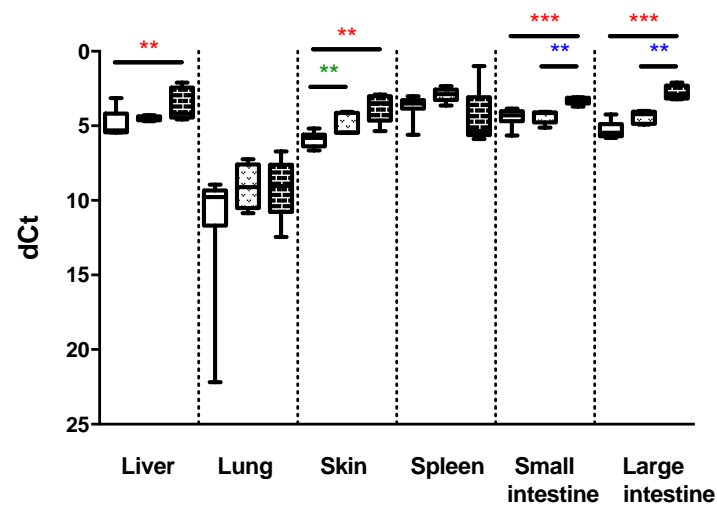

C

Cxcr4

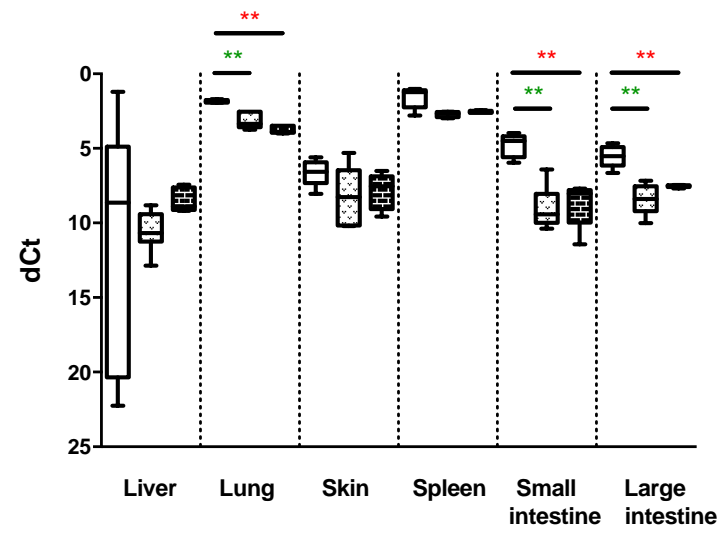

Figure 3.8: mRNA expression of chemokines $\mathrm{Cxcl15}$, $\mathrm{Cxcl16}$ and chemokine receptor $\mathrm{Cxcr} 4$ in different tissues in mice.

Relative mRNA expression chemokines of A. Cxcl15, B. Cxcl16 and chemokine receptor C. Cxcr4 in $\mathrm{aGvHD}$ mice $(\mathrm{n}=17)$ in comparison with normal healthy controls $(\mathrm{n}=15)(\mathrm{red})$, in aGvHD mice compared to BM control mice $(n=6)$ (blue) and preconditioning alone by comparing BM control mice to healthy controls (green). The $\mathrm{y}$-axis shows relative expression $(\mathrm{dCt}$ ), and $\mathrm{x}$ axis shows different mice groups in each tissue type. The p-values were calculated by Mann-Whitney U-test, and were corrected for FDR by Benjamin Hochberg correction.

$* \mathrm{P}<0.05, * * \mathrm{P}<0.01, * * * \mathrm{P}<0.001, * * * * \mathrm{P}<0.0001$ (significantly regulated genes in aGvHD mice compared to healthy controls)

$* \mathrm{P}<0.05, * * \mathrm{P}<0.01, * * * \mathrm{P}<0.001, * * * * \mathrm{P}<0.0001$ (significantly regulated genes in aGvHD mice compared to BM controls) 
$* \mathrm{P}<0.05, * * \mathrm{P}<0.01, * * * \mathrm{P}<0.001, * * * * \mathrm{P}<0.0001$ (significantly regulated genes in $\mathrm{BM}$ control mice due to preconditioning)

\subsubsection{Expression of the chemokine receptor $\mathrm{Cx} 3 \mathrm{cr} 1$ and its ligand $\mathrm{Cx} 3 \mathrm{cll}$ in mice}

We observed a strong upregulation of $C x 3 \mathrm{crl}$ mRNA in aGvHD mice compared to healthy controls in the spleen (13.34; $\mathrm{p}=0.016)$, small (49.71; $\mathrm{p}=0.008)$ and large (265.77; $\mathrm{p}=0.008)$ intestines (Figure 3.9A), whereas its ligand, Cx3cll was strongly downregulated in the aGvHD lung (0.47; $\mathrm{p}$ $=0.008)$ and large intestine $(\mathbf{0 . 3 6} ; \mathrm{p}=0.008)$ compared to healthy controls. Furthermore, $C x 3 c l 1$ was significantly reduced in aGvHD spleen $(\mathbf{0 . 1 5} ; \mathrm{p}=0.004)$ compared to BM controls (Figure 3.9B).

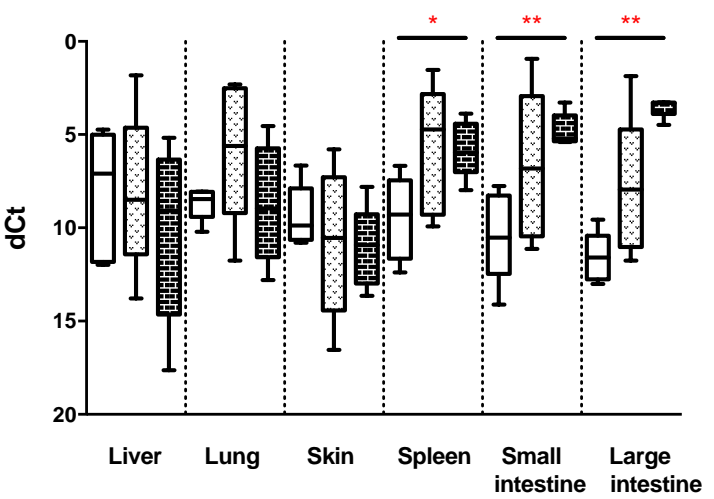

Cx3cr1
B

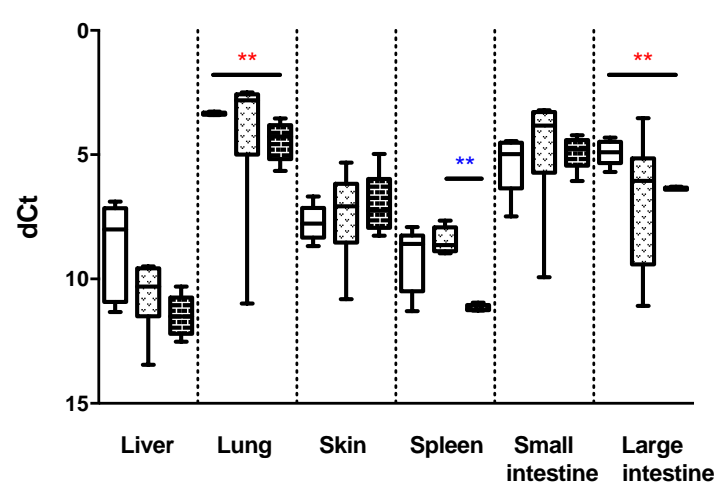

Figure 3.9: mRNA expression of chemokine receptor $C x 3 c r 1$ and its ligand $C x 3 c l 1$ in different tissues in mice.

Relative mRNA expression of chemokine receptor A. $C x 3 c r 1$ and its ligand B. $C x 3 c l 1$, in aGvHD mice ( $\mathrm{n}=$ 17) in comparison with normal healthy controls $(\mathrm{n}=15)$ (red), in aGvHD mice compared to BM control mice $(\mathrm{n}=6)$ (blue) and preconditioning alone by comparing BM control mice to healthy controls (green). The yaxis shows relative expression $(\mathrm{dCt})$, and $\mathrm{x}$ axis shows different mice groups in each tissue type. The $\mathrm{p}$ values were calculated by Mann-Whitney U-test, and were corrected for FDR by Benjamin Hochberg correction.

$* \mathrm{P}<0.05, * * \mathrm{P}<0.01, * * * \mathrm{P}<0.001,{ }^{*} * * * \mathrm{P}<0.0001$ (significantly regulated genes in aGvHD mice compared to healthy controls)

$* \mathrm{P}<0.05, * * \mathrm{P}<0.01, * * * \mathrm{P}<0.001,{ }^{*} * * * \mathrm{P}<0.0001$ (significantly regulated genes in aGvHD mice compared to BM controls)

$* \mathrm{P}<0.05, * * \mathrm{P}<0.01, * * * \mathrm{P}<0.001, * * * * \mathrm{P}<0.0001$ (significantly regulated genes in $\mathrm{BM}$ control mice due to preconditioning) 


\subsubsection{Expression of other genes}

IL-15 is a critical mediator for T cell function during aGvHD (Blaser et al., 2005). Il15 mRNA was not differentially regulated in any of the tissues, except it was significantly reduced in the large intestine $(\mathbf{0 . 4 4} ; \mathrm{p}=0.029)$ in aGvHD mice compared to healthy controls (Figure 3.10A). We found Interleukin-1 receptor-1 (Ilrl) mRNA to be significantly upregulated in the small intestine in mice with aGvHD (5.40; $\mathrm{p}=0.008)$ compared to BM controls. The expression of Illrl was also increased in the liver $(\mathbf{6 . 6 4} ; \mathrm{p}=0.001)$ and small intestine $(\mathbf{2 . 3 3} ; \mathrm{p}=0.007)$ of aGvHD mice compared to healthy controls (Figure 3.10B). Bone morphogenetic protein receptor type-1A (Bmplra) mRNA was significantly increased as a result of preconditioning in the liver $(\mathbf{2 . 7 6} ; \mathrm{p}=$ 0.001) and lung (7.99; $\mathrm{p}=0.002)$ in $\mathrm{BM}$ control mice compared to healthy controls, and its expression was further augmented in aGvHD mice in the liver $(\mathbf{3 . 1 5} ; \mathrm{p}=0.001)$ and lung (7.94; $\mathrm{p}=$ 0.019) compared to healthy controls (Figure 3.10C). BMPR1A is a receptor that belongs to a family of transmembrane serine-threonine kinases, its ligands are members of the TGF- $\beta$ superfamily. Ectonucleotide pyrophosphatase/ phosphodiesterase 1 (Enppl) mRNA was increased as a result of preconditioning in the large intestine $(\mathbf{2 . 5 5} ; \mathrm{p}=0.007)$ in $\mathrm{BM}$ controls compared to healthy controls, and in aGvHD small intestine $(3 ; p=0.042)$ compared to healthy controls. On the other hand, the expression of Enppl was significantly decreased in the aGvHD liver $(\mathbf{0 . 0 7} ; \mathrm{p}=$ 0.042) and lung $(\mathbf{0 . 1 7} ; \mathrm{p}=0.042)$ compared to healthy controls (Figure 3.10D). ENPP1 has a broad specificity and cleaves a number of substrates such as phosphodiester bonds of nucleic acids. Fc fragment of immunoglobulin epsilon receptor subunit gamma (FCER1G) is involved in transmembrane signalling receptor activity and binding of IgE. Fcerlg mRNA was significantly increased in the spleen $(\mathbf{2 . 4 6} ; \mathrm{p}=0.004)$ as due to preconditioning in BM controls and in aGvHD liver $(\mathbf{2 . 3 1} ; \mathrm{p}=0.012)$, skin $(\mathbf{6 . 9 2} ; \mathrm{p}=0.002)$ and large intestine $(\mathbf{2 . 1 5} ; \mathrm{p}=0.001)$ compared to healthy controls (Figure 3.10E). Low affinity immunoglobulin gamma Fc region receptor III (FCGR3) is required for NK cell-mediated antibody-dependent cytotoxicity and phagocytosis by macrophages. On the other hand, we observed no change in expression patterns of Fcgr3 in any of the tissues (Figure 3.10F). The Intercellular adhesion molecule 1 (ICAM-1) is expressed on endothelial cells and is critical for the migration of leukocytes to tissues during inflammation (Ren et al., 2010). Icaml mRNA was significantly increased in the liver (5.66; p = 0.001), skin (10.13; $p$ $=0.001)$, small $(\mathbf{4 . 1 3} ; \mathrm{p}=0.001)$ and large $(\mathbf{1 0 . 5 5} ; \mathrm{p}=0.001)$ intestines of aGvHD mice compared to healthy controls. Preconditioning had no significant effect on the regulation of Icaml (Figure $3.10 \mathrm{G})$. 
A

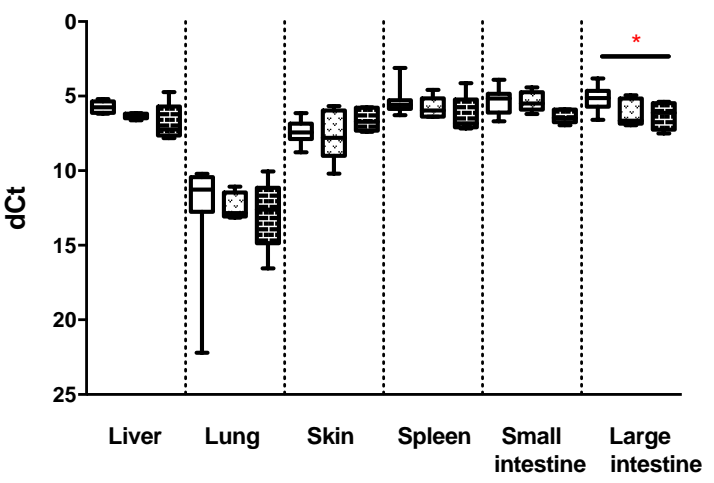

C

Bmpr1a

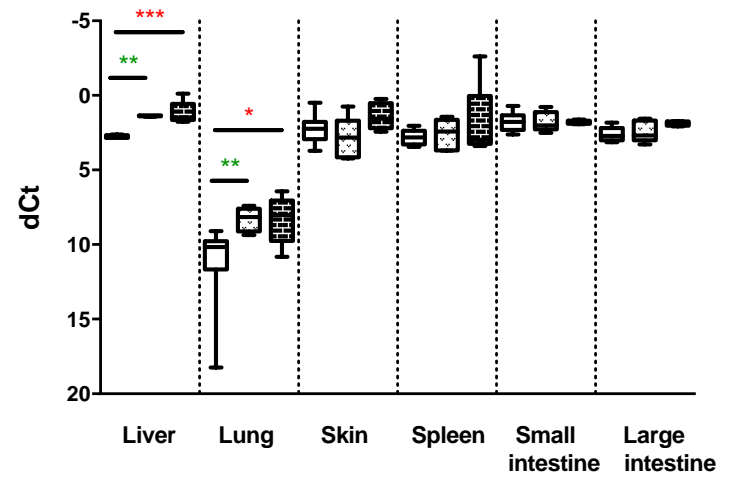

E

Fcer1g

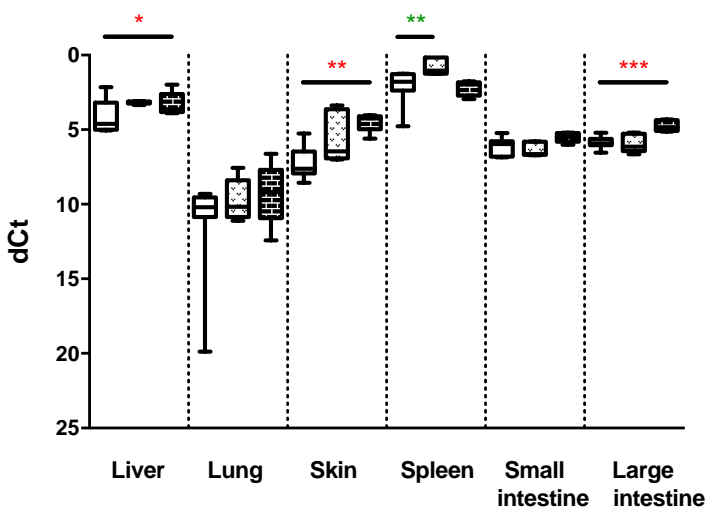

B

II1r1

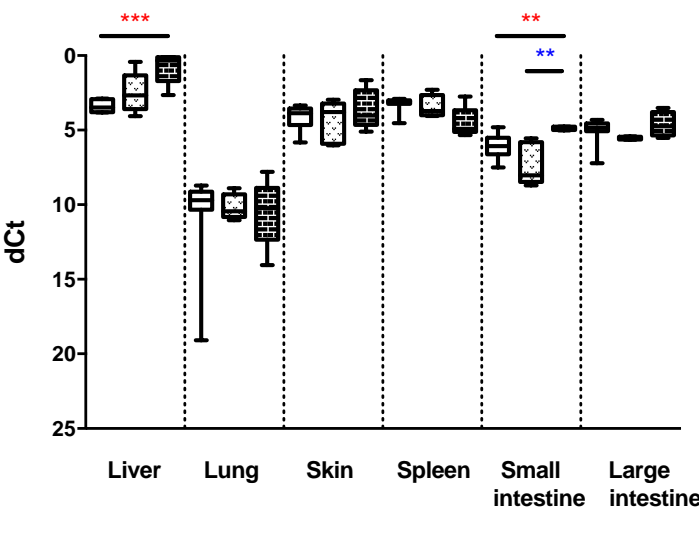

D

Enpp1

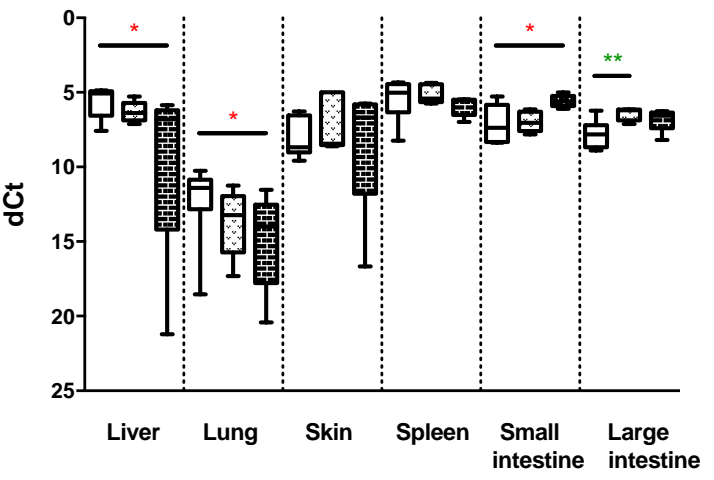

Fcgr3

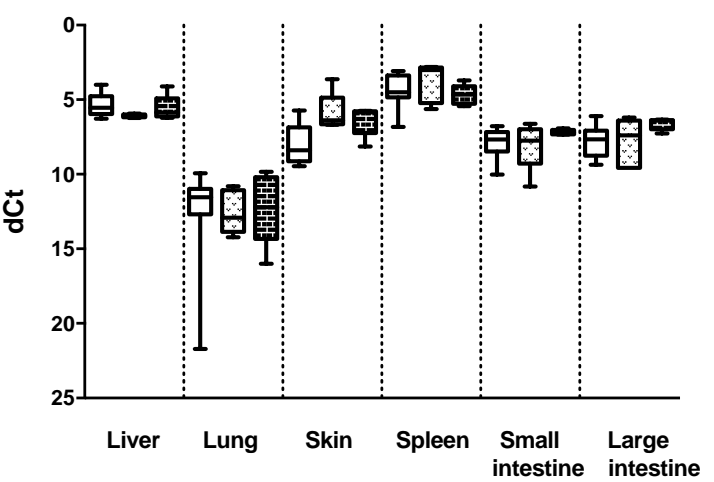




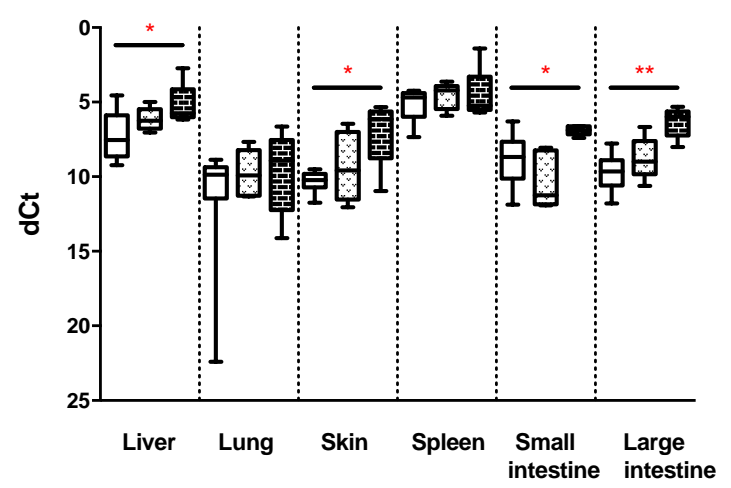

Figure 3.10: mRNA expression other genes involved in immune responses in different tissues in mice.

Relative mRNA expression of other genes involved in immune responses, in aGvHD mice $(n=17)$ in comparison with normal healthy controls $(\mathrm{n}=15)(\mathrm{red})$, in aGvHD mice compared to BM control mice $(\mathrm{n}=6)$ (blue) and preconditioning alone by comparing BM control mice to healthy controls (green). The y-axis shows relative expression $(\mathrm{dCt})$, and $\mathrm{x}$ axis shows different mice groups in each tissue type. The $\mathrm{p}$-values were calculated by Mann-Whitney U-test, and were corrected for FDR by Benjamin Hochberg correction. $* \mathrm{P}<0.05, * * \mathrm{P}<0.01, * * * \mathrm{P}<0.001$, **** $\mathrm{P}<0.0001$ (significantly regulated genes in aGvHD mice compared to healthy controls)

$* \mathrm{P}<0.05, * * \mathrm{P}<0.01, * * * \mathrm{P}<0.001, * * * * \mathrm{P}<0.0001$ (significantly regulated genes in aGvHD mice compared to BM controls)

$* \mathrm{P}<0.05, * * \mathrm{P}<0.01, * * * \mathrm{P}<0.001, * * * * \mathrm{P}<0.0001$ (significantly regulated genes in $\mathrm{BM}$ control mice due to preconditioning)

In summary, we observed an observed upregulation of the Th1, Th2 and Th17 cytokines, genes associated with Tregs, as well as the adhesion molecule Icaml, and several other chemokines including chemokine receptor Cxcr3 and its ligands Cxcl9, Cxcl10 and Cxcl11, chemokine receptors $C c r 1, C c r 5, C x 3 c r 1$ and chemokines $C c l 9$ and $C x c l 16$ in the different tissues in aGvHD mice compared to the healthy control mice. This is possibly due to activation of alloreactive $\mathrm{T}$ cells in the target organs or $\mathrm{T}$ cell infiltration in sites of inflammation. Other chemokines, Cxcl15, Cx3cll, Ccl4, chemokine receptors Ccr4, Cxcr4 and the cytokine Ill5 were significantly downregulated. Overall, the genes were differentially expressed across the different target organs, however the most profound change in gene regulation was observed in the intestine. 


\subsection{Gene expression of previously identified candidate genes in mice and rat aGvHD}

\subsubsection{Expression of genes regulated by IFN- $\gamma$}

Of the candidate genes previously identified by Novota and colleagues, several genes are regulated by the IFN- $\gamma$, suggesting their role in inflammation during aGvHD pathogenesis.

\subsubsection{Mice}

Interestingly, Lilra5 was significantly increased during aGvHD in all the tissues. Leukocyte immunoglobulin-like receptor subfamily A member 5 (LILRA5) is an activating Ig-like receptor, expressed by immune cells and is associated with the release of pro-inflammatory cytokines (Brown et al., 2004). In aGvHD mice compared to healthy controls, Lilra5 was significantly upregulated in the liver $(\mathbf{6 . 8 4} ; \mathrm{p}=0.0001)$, lung $(\mathbf{4 . 0 1} ; \mathrm{p}=0.007)$, skin $(\mathbf{1 2 8 . 6} ; \mathrm{p}=0.02)$, spleen (8.58; $\mathrm{p}=0.001)$, small $\left(\mathbf{9 . 8} \times \mathbf{1 0}^{2} ; \mathrm{p}=0.0001\right)$ and large (168.91; $\left.\mathrm{p}=0.002\right)$ intestines. Moreover, the expression of Lilra5 was also significantly increased in the $\mathrm{BM}$ control spleen due to preconditioning $(\mathbf{8 . 1} ; \mathrm{p}=0.005)$ and in $\mathrm{aGvHD}$ small intestine $(\mathbf{1 3 4 . 3 4} ; \mathrm{p}=0.015)$ compared to BM controls (Figure 3.11A). Similarly, Lstl was significant increased in aGvHD mice lung (9.63; $\mathrm{p}=0.002)$, skin (8.38; $\mathrm{p}=0.007)$, spleen (65.59; $\mathrm{p}=0.0001)$, small (734.91; $\mathrm{p}=0.0001)$ and large (37.68; $\mathrm{p}=0.016$ ) intestines compared to healthy controls. Furthermore, the expression of Lst 1 was upregulated in the BM control spleen due to preconditioning $(\mathbf{4 1 . 0 7} ; \mathrm{p}=0.0001)$ compared to healthy controls, and between aGvHD and BM control mice, in both the small (5.48; $p=0.02)$ and large (3.2; $\mathrm{p}=0.013$ ) intestines (Figure 3.11B). Leukocyte-specific transcript 1 (LST1) is encoded within class III region of the MHC, (de Baey et al., 1997) and has been implicated in inflammatory and infectious diseases (Mulcahy et al., 2006).

It has previously been reported that MSR1 is involved in the regulation of anti-inflammatory responses (Fulton et al., 2006). Msrl mRNA was also increased in all tissues except the liver. In aGvHD mice compared to healthy controls, $M s r l$ was strongly upregulated in the lung $(5.29 ; \mathrm{p}=$ 0.018), skin (106.48; $\mathrm{p}=0.0001)$, spleen $(\mathbf{9 . 3 4} ; \mathrm{p}=0.0001)$, small $(\mathbf{9 . 6 5} ; \mathrm{p}=0.0001)$ and large (13.69; $\mathrm{p}=0.001)$ intestines. In addition, $M s r l$ was also increased in the spleen $(\mathbf{8 . 1 3} ; \mathrm{p}=0.003)$ of BM control mice compared to healthy controls as a result of preconditioning (Figure 3.11C). We observed an increased expression of Ptger 2 mRNA in the large intestine $(\mathbf{3 . 2} ; \mathrm{p}=0.013)$ in mice with aGvHD compared to BM controls. Prostaglandin E receptor 2 (PTGER2) or Prostaglandin E2 can modulate cytokine responses by $\mathrm{CD} 4^{+} \mathrm{T}$ cells directly, and can increase the expression of IL-17 
and decreased IFN- $\gamma$ production by acting on T cells directly (Napolitani et al., 2009) and inhibit T cell responses by blocking the proliferation of $T$ cells (Harris et al., 2002). In addition, PTGER2 was also associated with an enhanced secretion of IL-23 by DCs (Weaver et al., 2007). Furthermore, Ptger2 mRNA was significantly increased in aGvHD small $(\mathbf{1 . 7 8} ; \mathrm{p}=0.02)$ and large (1.91; $p=0.025)$ intestines and reduced in spleen $(\mathbf{0 . 3 9} ; \mathrm{p}=0.004)$ compared to healthy control mice (Figure 3.11D).

We observed an increased expression of Tapl in BM control mice due to preconditioning in the lung $(\mathbf{6 0 3 . 4 5} ; \mathrm{p}=0.003)$ and liver $(\mathbf{1 2 9 . 2 2} ; \mathrm{p}=0.005)$ compared to healthy controls, whereas its expression was significantly reduced in aGvHD liver $(\mathbf{0 . 0 0 1} ; \mathrm{p}=0.002)$ compared to BM controls and in aGvHD spleen compared to healthy controls $(\mathbf{0 . 0 1} ; \mathrm{p}=0.004)$ (Figure 3.11E).

Trem 2 was significantly downregulation in aGvHD lung $(\mathbf{0 . 1 0} ; \mathrm{p}=0.001)$, spleen $(\mathbf{0 . 0 7} ; \mathrm{p}=0.001)$ and small intestine $\left(\mathbf{0 . 1} \times \mathbf{1 0}^{-4} ; \mathrm{p}=0.001\right)$ compared to healthy controls, and in aGvHD liver $(\mathbf{0 . 0 4}$; $\mathrm{p}=0.013)$ compared to BM controls. In contrast, preconditioning in the BM control liver (393.05; $\mathrm{p}=0.005)$ as well as aGvHD liver $(\mathbf{1 6 . 4 8} ; \mathrm{p}=0.008)$, showed a strong increase expression of Trem 2 compared to healthy controls (Figure 3.11F). TREM2 is downregulated by IFN- $\gamma$ (Zhao and Ivashkiv, 2011).

The gene expression of $U b d$ was strongly increased in all the target tissues of aGvHD, in the liver (2.17; $\mathrm{p}=0.0001)$, lung (10.32; $\mathrm{p}=0.0001)$, skin (59.3; $\mathrm{p}=0.0001)$, spleen (59.3; $\mathrm{p}=0.001)$, small (11.25; $\mathrm{p}=0.0001)$ and large intestines $(\mathbf{7 . 5 5} ; \mathrm{p}=0.0001)$ and in aGvHD mice compared to BM controls in the liver (2.17; $p=0.004)$, lung (10.32; $p=0.016)$, skin (59.3; $p=0.0001)$, small (11.25; $\mathrm{p}=0.001)$ and large intestines (7.55; $\mathrm{p}=0.0001)$. Moreover, Ubd was also highly increased in the liver $(\mathbf{2 8 . 7 1} ; \mathrm{p}=0.0001)$ and lung $(\mathbf{1 0 . 6 5} ; \mathrm{p}=0.014)$ of BM control mice compared to healthy controls due to preconditioning (Figure 3.11G). UBD is a downstream regulator of Tregs, (Ocklenburg et al., 2006) and is regulated by IFN- $\gamma$. 
A

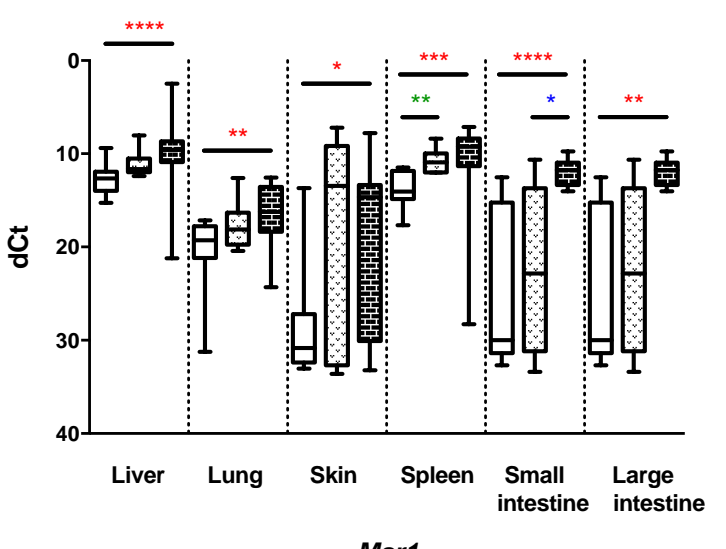

C

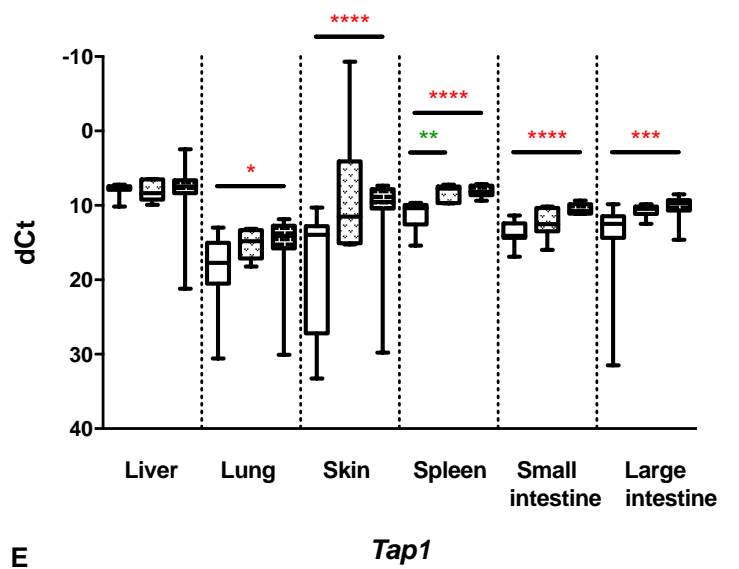

B

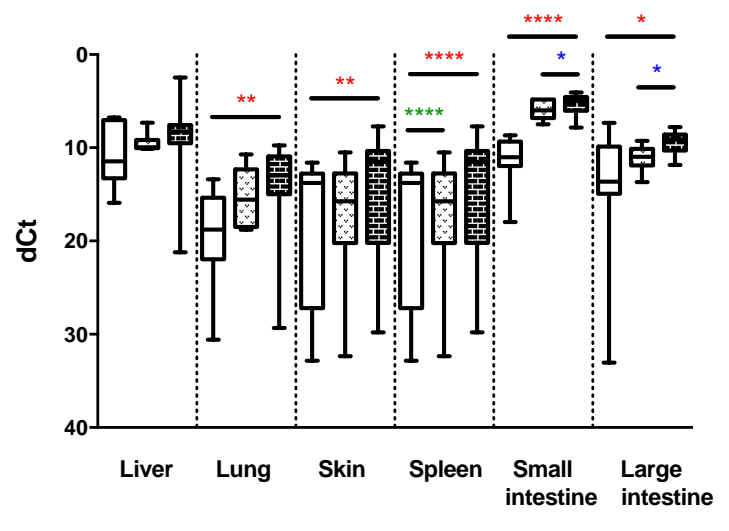

D

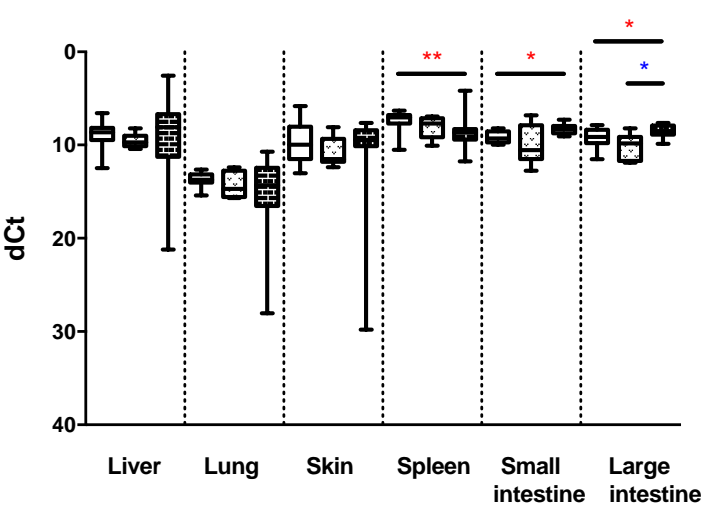

F

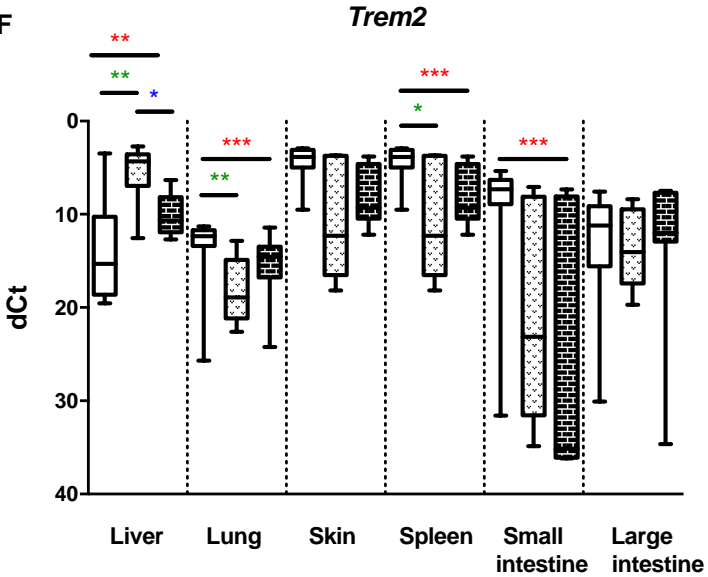


G

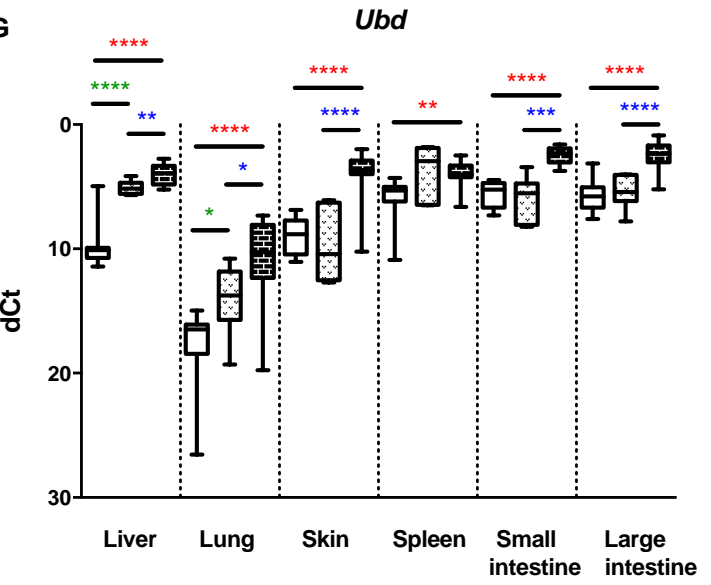

Figure 3.11: mRNA expression of candidate genes regulated by IFN- $\gamma$ in different tissues in mice.

Relative mRNA expression of candidate genes regulated by IFN $\gamma$, in aGvHD mice $(\mathrm{n}=17)$ in comparison with normal healthy controls $(n=15)(r e d)$, in aGvHD mice compared to BM control mice $(n=6)$ (blue) and preconditioning alone by comparing BM control mice to healthy controls (green). The y-axis shows relative expression $(\mathrm{dCt})$, and $\mathrm{x}$ axis shows different mice groups in each tissue type. The $\mathrm{p}$-values were calculated by Mann-Whitney U-test, and were corrected for FDR by Benjamin Hochberg correction.

$* \mathrm{P}<0.05, * * \mathrm{P}<0.01, * * * \mathrm{P}<0.001$, **** $\mathrm{P}<0.0001$ (significantly regulated genes in aGvHD mice compared to healthy controls)

$* \mathrm{P}<0.05, * * \mathrm{P}<0.01, * * * \mathrm{P}<0.001, * * * * \mathrm{P}<0.0001$ (significantly regulated genes in aGvHD mice compared to BM controls)

$* \mathrm{P}<0.05, * * \mathrm{P}<0.01, * * * \mathrm{P}<0.001,{ }^{*} * * * \mathrm{P}<0.0001$ (significantly regulated genes in $\mathrm{BM}$ control mice due to preconditioning).

\subsubsection{Rats}

In the aGvHD rats, these genes were not as differentially expressed. Lilra5, Msr1, Lst1 and Trem2 were not regulated in aGvHD mice compared to syngeneic control mice. The expression of Lilra5 (Figure 3.12A) and Lst 1 (Figure 3.12B) showed a trend of upregulation in the liver, lung and small intestine, same as in mice. In addition, we observed a trend of upregulation of Msrl (Figure 3.12C) and Trem 2 (Figure 3.12F) in the rat liver, in line with their regulation in mice, but their expression trends in the other tissues were different. On the other hand, Ptger2 was increased significantly in aGvHD liver alone (6.98; $\mathrm{p}=0.007$ ) (Figure 3.12D). Similarly, the expression of Tap1 mRNA was significantly increased in the liver $\mathbf{2 . 9 8 ;} \mathrm{p}=0.0069)$, as seen in aGvHD mice as well (Figure 3.12E). Moreover, $U b d$ was strongly upregulated in the lung $(\mathbf{5 8 . 5 8} ; \mathrm{p}=0.0001)$ and the small intestine (30.85; $\mathrm{p}=0.0036$ ) in aGvHD rat compared to the syngeneic controls (Figure $3.12 \mathrm{G}$ ). Moreover, $U b d$ showed an upregulated trend of expression in the liver and skin as well, similar to that in mice. 
A

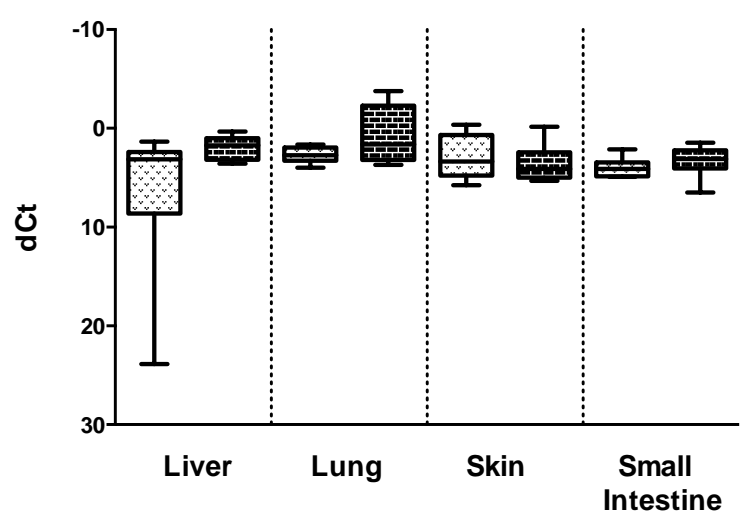

C

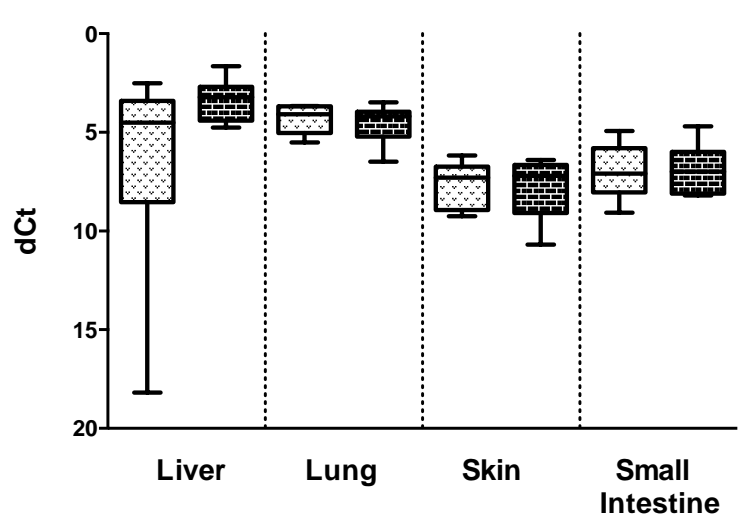

E

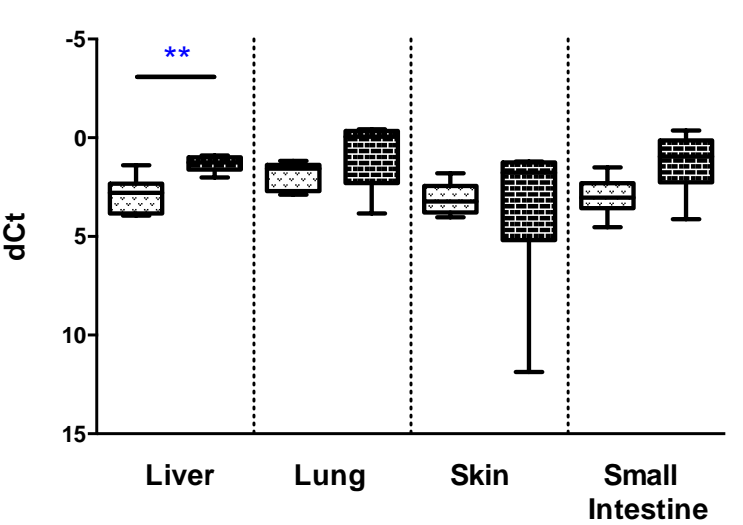

B

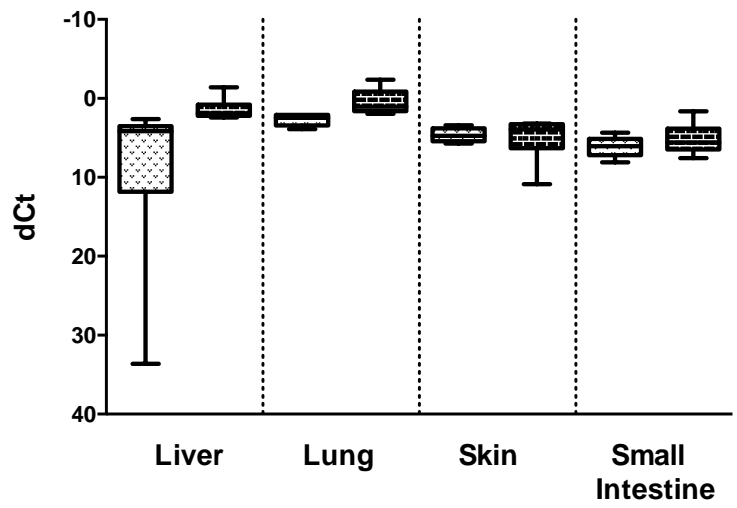

D

Ptger2

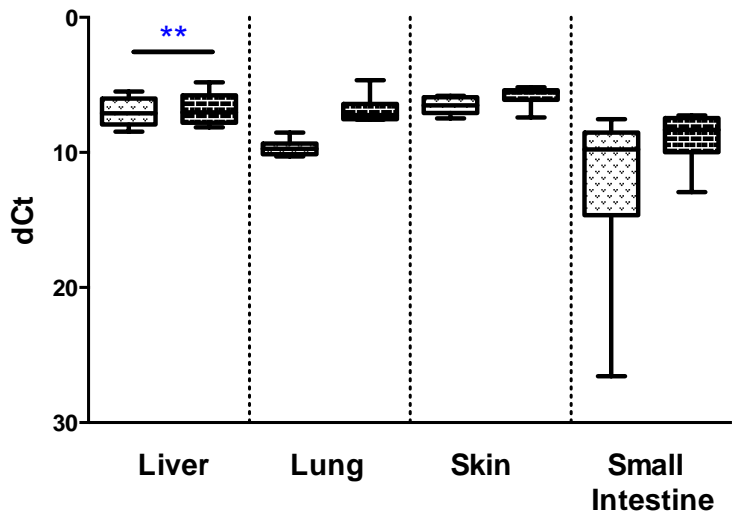

F

Trem2

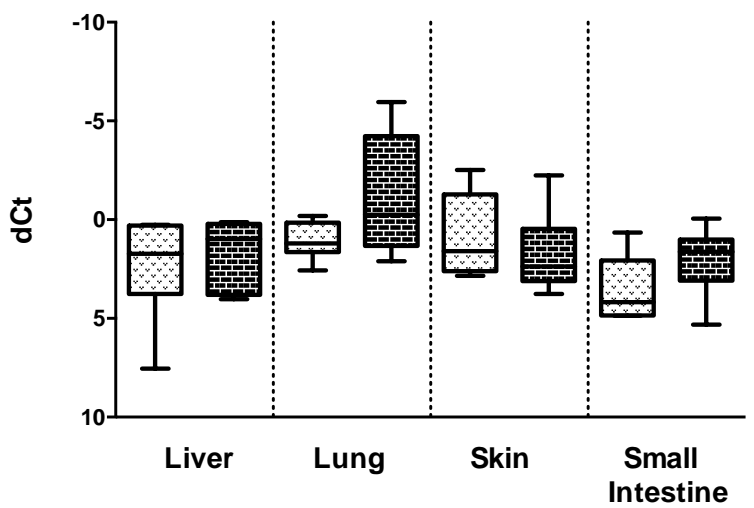




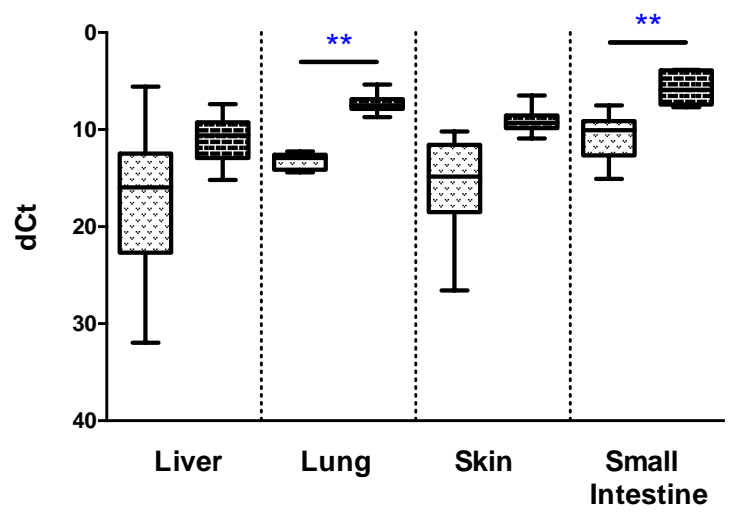

Figure 3.12: mRNA expression of genes regulated by IFN- $\gamma$ in different tissues in rats. Relative mRNA expression of genes regulated by IFN- $\gamma$ in rats with aGvHD $(n=6)$, compared to syngeneic controls $(n=6)$. The $y$-axis shows relative expression $(\mathrm{dCt})$, and $\mathrm{x}$-axis shows control rat and aGvHD expression in different tissues. The p-values were calculated by Mann-Whitney U-test, and were corrected for FDR by Benjamin Hochberg correction.

$* \mathrm{P}<0.05, * * \mathrm{P}<0.01, * * * \mathrm{P}<0.001, * * * * \mathrm{P}<0.0001$

\subsubsection{Expression of genes regulated by $B$ and $T$ cell activation}

\subsubsection{Mice}

Capsase recruitment domain 11 (CARD11) is critical for antigen receptor signalling (Pomerantz et al., 2002). Cardl1 mRNA was significantly decreased in the spleen $(\mathbf{0 . 3 8} ; \mathrm{p}=0.012)$ due to preconditioning in $\mathrm{BM}$ control mice, and was further reduced as a result of aGvHD in the spleen $\mathbf{( 0 . 3 3} ; \mathrm{p}=0.0001)$ and small intestine $(\mathbf{0 . 5 2} ; \mathrm{p}=0.007)$ compared to BM controls. Moreover, the overall expression of Card11 was also significantly decreased in aGvHD mice compared to healthy controls in the spleen $\left(\mathbf{0 . 2} \times \mathbf{1 0}^{-3} ; \mathrm{p}=0.0001\right)$, small $(\mathbf{0 . 2 3} ; \mathrm{p}=0.0001)$ and large $(\mathbf{0 . 3 1} ; \mathrm{p}=0.0001)$ intestines (Figure 3.13A). The lack of Hematopoietic cell-specific Lyn substrate 1 (HCLS1) on B and $\mathrm{T}$ cells causes a defect in proliferation and antigen receptor induced apoptosis (Fukuda et al., 1995). Hcls I mRNA was significantly increased in the liver (3.95; $\mathrm{p}=0.0001)$, lung (8.61; $\mathrm{p}=$ 0.001), skin (44.29; $p=0.0001)$ and large intestine $(3.18 ; p=0.001)$ in aGvHD mice compared to healthy controls (Figure 3.13B). Moreover, preconditioning significantly increased the expression of Hclsl in the liver $(\mathbf{1 . 8 2} ; \mathrm{p}=0.011)$ and lung $(\mathbf{9 . 9} ; \mathrm{p}=0.006)$ in $\mathrm{BM}$ control mice compared to healthy mice, as well as in aGvHD small $(\mathbf{3 . 2 8} ; \mathrm{p}=0.002)$ and large $(\mathbf{2 . 4 7} ; \mathrm{p}=0.018)$ intestines compared to BM controls. 
A

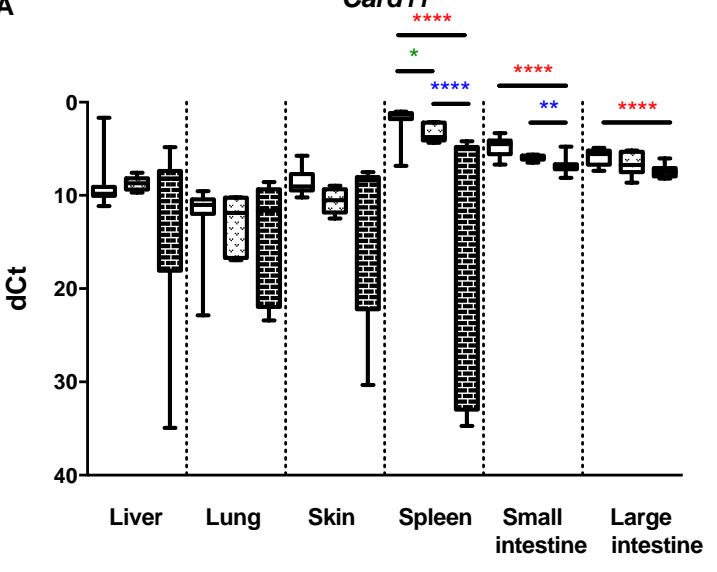

C

Pik3ap1

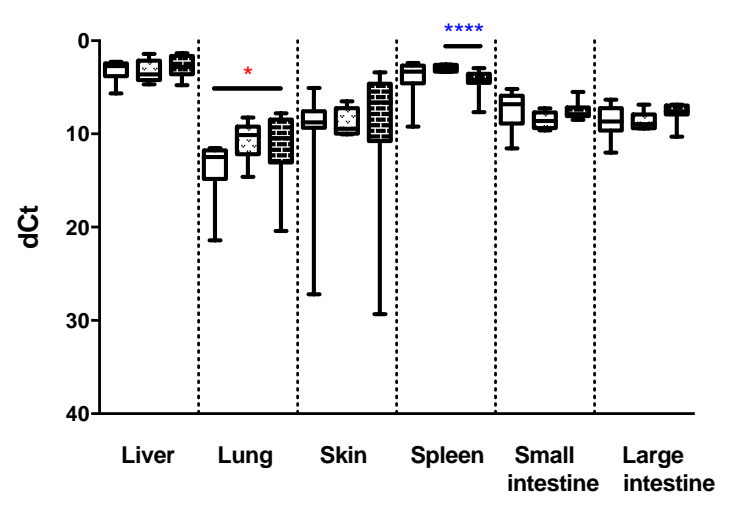

Hcls1
D
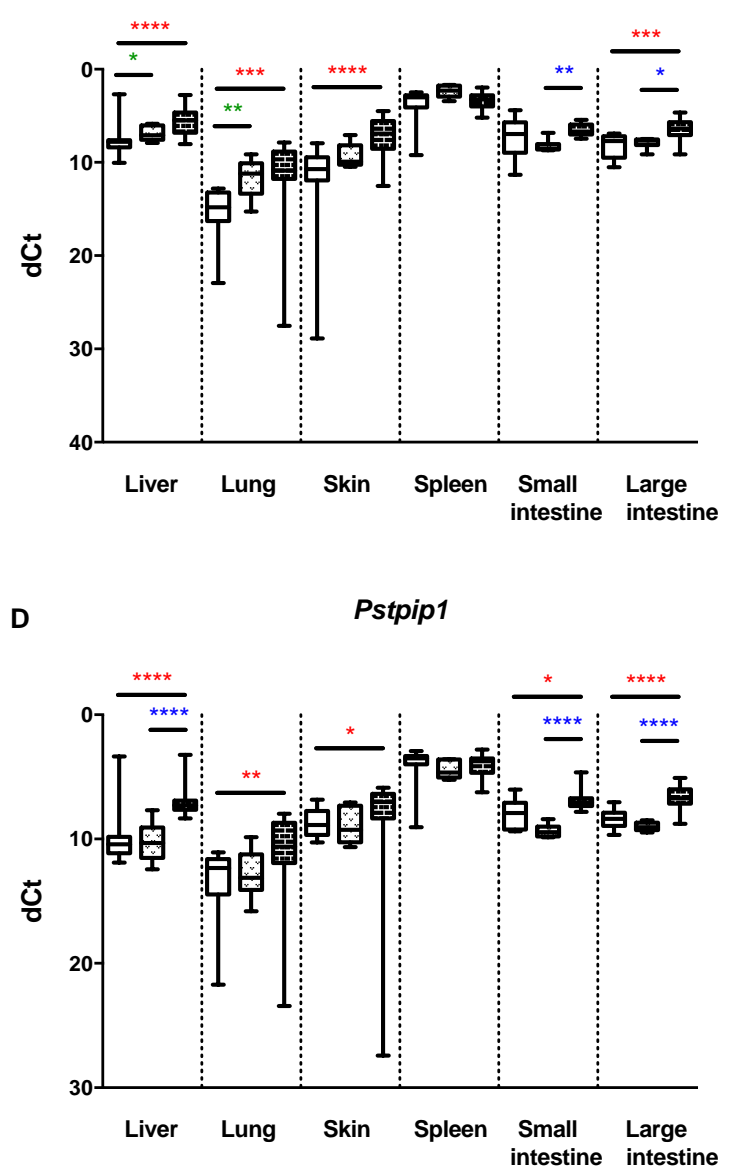

E

Ptpn7

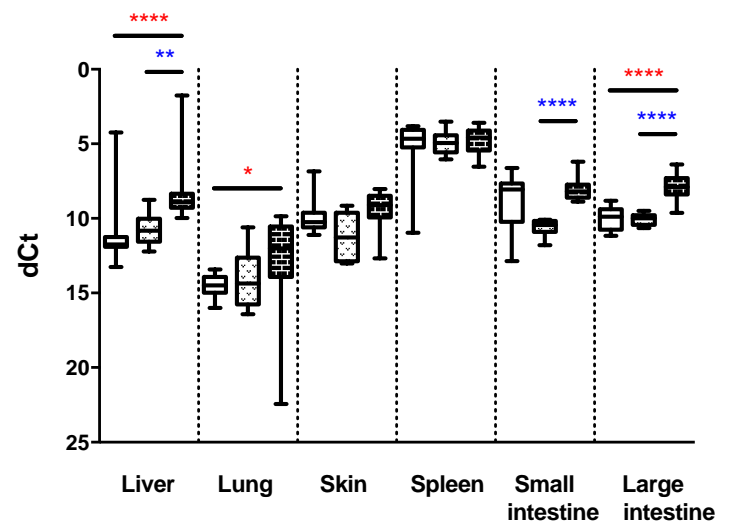

Figure 3.13: mRNA expression of candidate genes regulated by activation of $B$ and $T$ cells in different tissues in mice.

Relative mRNA expression of candidate genes regulated by activation of B and T cells, in aGvHD mice ( $\mathrm{n}=$ 17 ) in comparison with normal healthy controls $(\mathrm{n}=15)$ (red), in aGvHD mice compared to BM control mice $(n=6)$ (blue) and preconditioning alone by comparing BM control mice to healthy controls (green). The yaxis shows relative expression $(\mathrm{dCt})$, and $\mathrm{x}$ axis shows different mice groups in each tissue type. The $\mathrm{p}$ - 
values were calculated by Mann-Whitney U-test, and were corrected for FDR by Benjamin Hochberg correction.

$* \mathrm{P}<0.05, * * \mathrm{P}<0.01, * * * \mathrm{P}<0.001, * * * * \mathrm{P}<0.0001$ (significantly regulated genes in aGvHD mice compared to healthy controls)

$* \mathrm{P}<0.05, * * \mathrm{P}<0.01, * * * \mathrm{P}<0.001, * * * * \mathrm{P}<0.0001$ (significantly regulated genes in aGvHD mice compared to BM controls)

$* \mathrm{P}<0.05, * * \mathrm{P}<0.01, * * * \mathrm{P}<0.001, * * * * \mathrm{P}<0.0001$ (significantly regulated genes in $\mathrm{BM}$ control mice due to preconditioning)

Phosphoinositde-3-kinase adaptor protein 1 (PIK3AP1) is involved in the development of B cells (Yamazaki and Kurosaki, 2003) and activation of NK cells (Ni et al., 2012). Pik3apl was not regulated in many of the aGvHD target tissues, but was significantly decreased in the aGvHD spleen (0.36; $\mathrm{p}=0.0001)$ compared to BM controls and increased in the aGvHD lung $(4.04 ; p=$ 0.016) compared to healthy controls (Figure 3.13C). On the other hand, Proline serine threonine phosphatase interacting protein 1 (Pstpipl) was significantly increased in all the aGvHD target tissues. In aGvHD mice compared to BM controls, Pstpipl was significantly increased in the liver (10.73; $\mathrm{p}=0.0001)$, small (5.66; $\mathrm{p}=0.0001)$ and large intestine (5.13; $\mathrm{p}=0.0001)$. Pstpip1 was further upregulated in aGvHD mice compared to healthy controls in the liver $(\mathbf{9 . 4 7} ; p=0.0001)$, lung (1.66; $p=0.005)$, skin (1.15; $p=0.013)$, small $(2.12 ; p=0.038)$ and large $(4.34 ; p=0.0001)$ intestines (Figure 3.13D). PSTPIP1 is involved in the downregulation of CD2-triggered adhesion and activation of T cells ( $\mathrm{Li}$ et al., 1998). Similarly, Protein tyrosine phosphatase non-receptor type 7 (Ptpn7) was significantly upregulated in the liver $(\mathbf{1 0 . 7 3} ; \mathrm{p}=0.0001)$, small $(\mathbf{5 . 6 6} ; \mathrm{p}=0.0001)$ and large intestine $(\mathbf{5 . 1 3} ; \mathrm{p}=0.0001)$ in aGvHD mice compared to BM controls. Its expression was further increased in aGvHD mice compared to healthy controls in the liver $(\mathbf{8 . 1 4} ; p=0.0001)$, lung $(\mathbf{1 . 1 9} ; \mathrm{p}=0.027)$ and large intestine $(4.09 ; \mathrm{p}=0.0001)$ (Figure 3.13E). PTPN7 is involved in $\mathrm{T}$ and B lymphocyte development and signal transduction (Saxena et al., 1998).

\subsubsection{Rats}

In rat aGvHD liver, we observed an increase in the expression of Hcls1 (5.28; $\mathrm{p}=0.0069$ ) (Figure 3.14A) and Ptpn7 compared to $(3.67 ; \mathrm{p}=0.004)$ (Figure 3.14D) gene expression was observed in rats with aGvHD. The mRNA expression of Pik3apland Pstpipl was not differentially regulated in the rat. However, a similar trend of upregulation to mice was observed for Pik3apl (Figure 3.4B) in the aGvHD liver and small intestine, and for Pstpipl (Figure 3.14C) in all the tissues except skin. 
A

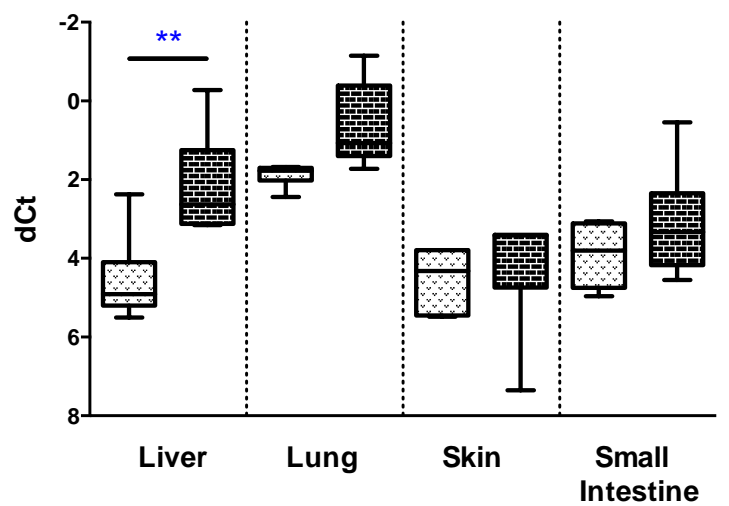

C

Pstpip1

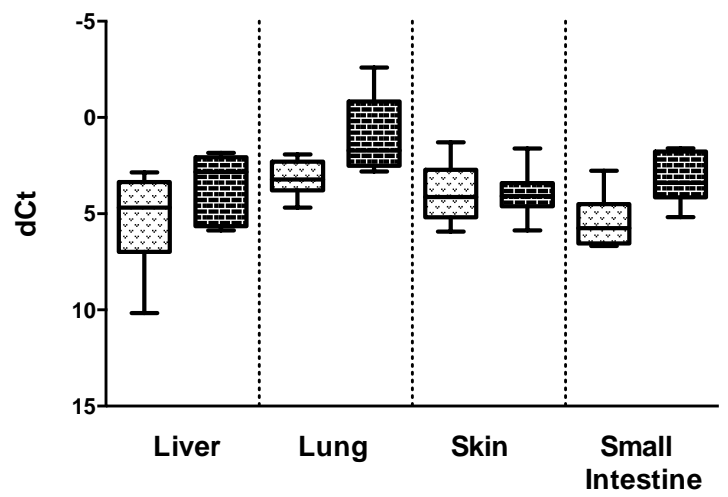

B

Pik3ap1

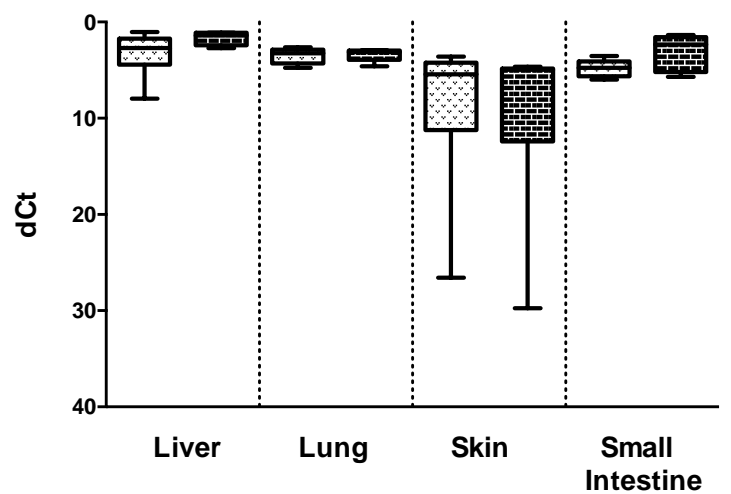

D

Ptger2

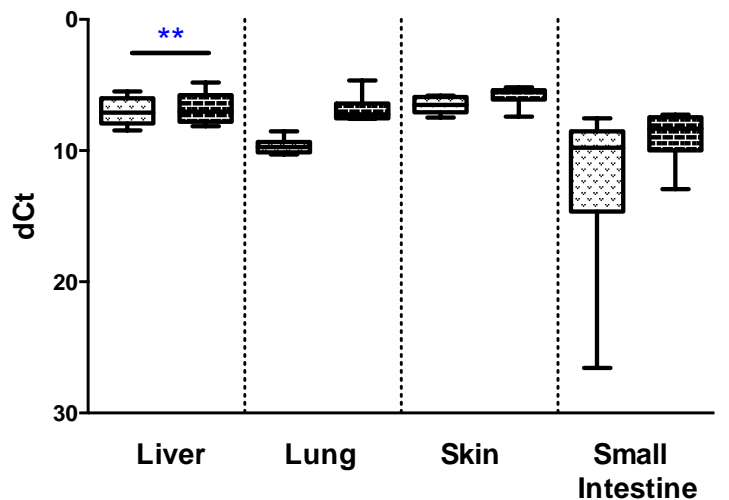

Figure 3.14: mRNA expression of genes regulated by $B$ and $T$ cell activation in different tissues in rats.

Relative mRNA expression of genes regulated by $B$ and $T$ cell activation in rats with aGvHD, $(n=6)$ on comparison with syngeneic controls $(\mathrm{n}=6)$. The $\mathrm{y}$-axis shows relative expression $(\mathrm{dCt})$, and $\mathrm{x}$-axis shows control rat and aGvHD expression in different tissues. The $\mathrm{P}$ values were calculated by Mann-Whitney U-test, and were corrected for FDR by Benjamin Hochberg correction.

$* \mathrm{P}<0.05, * * \mathrm{P}<0.01, * * * \mathrm{P}<0.001, * * * * \mathrm{P}<0.0001$ 


\subsubsection{Expression of genes regulated by innate immune responses}

\subsubsection{Mice}

No significant changes in the expression of Acidic Nuclear Phosphoprotein 32 Family, Member A (Anp32a) mRNA was observed in the mice during aGvHD. ANP32A is protein coding gene involved in several cellular processes including proliferation, differentiation and apoptosis. It is also implicated in tumor suppression, by stimulating apoptosis (Pan et al., 2009). Anp32a mRNA was decreased in the liver due to preconditioning $(0.06 ; p=0.014)$, but remained unchanged in the other tissues (Figure 3.15A).

C1q and Tumor Necrosis Factor Related Protein 7 (C1QTNF7) is a protein coding gene that is indirectly impacted by NR3C1 protein ( $\mathrm{Lu}$ et al., 2007), the glucocorticoid receptor gene is involved in cellular proliferation and inflammatory responses (Ray and Prefontaine, 1994). The mRNA expression of Clqtnf7 was strongly increased in the skin $(\mathbf{2 . 7} ; \mathrm{p}=0.008)$, but reduced in the spleen $(\mathbf{0 . 0 1} ; \mathrm{p}=0.0001)$ and large intestine $\left(\mathbf{0 . 3} \times \mathbf{1 0}^{-3} ; \mathrm{p}=0.002\right)$ in aGvHD mice compared to healthy controls. In the spleen, Clqtnf7 was further reduced in aGvHD mice $(\mathbf{0 . 1 1} ; \mathrm{p}=0.008)$ compared to BM controls. No change in Clqtnf7 expression was observed in the aGvHD liver, however it was strongly reduced in the $\mathrm{BM}$ control liver as a result of preconditioning $(\mathbf{0 . 0 0 1} ; \mathrm{p}=$ 0.0001) compared to BM controls (Figure 3.15B).

Induction of High-Temperature Requirement A Serine Peptidase 1 (HTRA1) in the presence of LPS, increases the incidence of collagen-induced arthritis in mice (Hou et al., 2013). Moreover TLR-4 ligands induce the expression of HTRA1 in macrophages and fibroblasts (Hou et al., 2013). Htral was significantly reduced in the aGvHD spleen $(\mathbf{0 . 4 9} ; \mathrm{p}=0.001)$ compared to healthy controls and decreased in the aGvHD spleen $(\mathbf{0 . 3 3} ; \mathrm{p}=0.0001)$ and small intestine $(\mathbf{2 . 6 4} ; \mathrm{p}=$ $0.0001)$ but increased in the large intestine $(1.71 ; \mathrm{p}=0.018)$ compared to BM controls. In the liver however, Htral was downregulated in BM control mice due to preconditioning $(\mathbf{0 . 4} ; \mathrm{p}=0.0001)$, but significantly increased in aGvHD mice $(\mathbf{4 0 . 4} ; \mathrm{p}=0.002)$ compared to the BM controls (Figure 3.15C). 
A

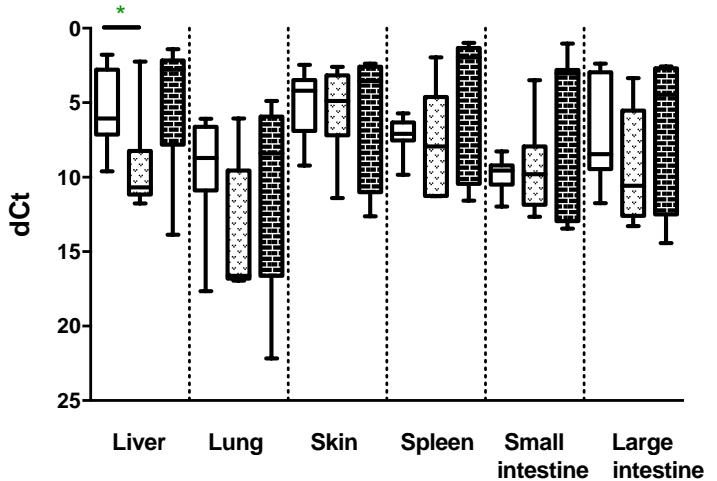

C

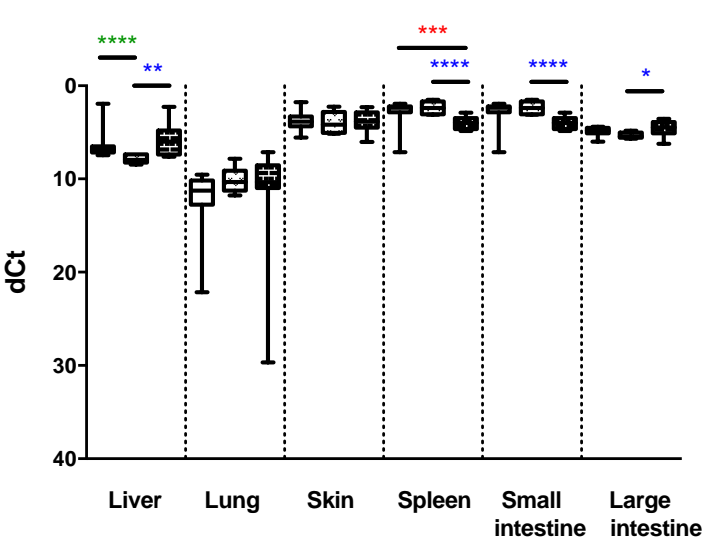

B

C1qtnf7

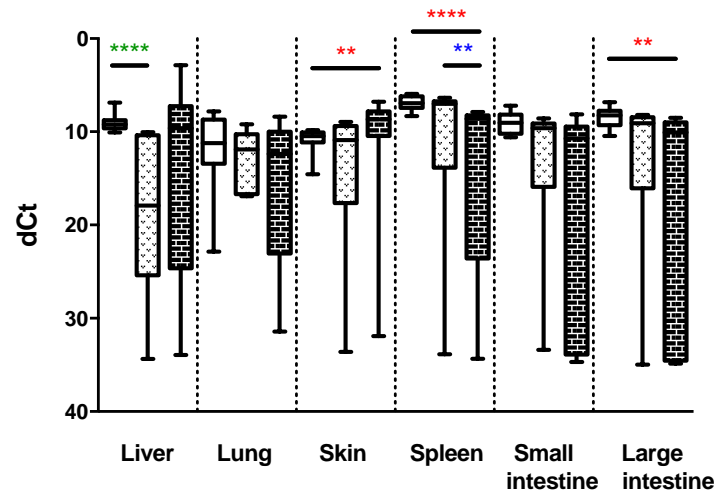

D

Lgals7

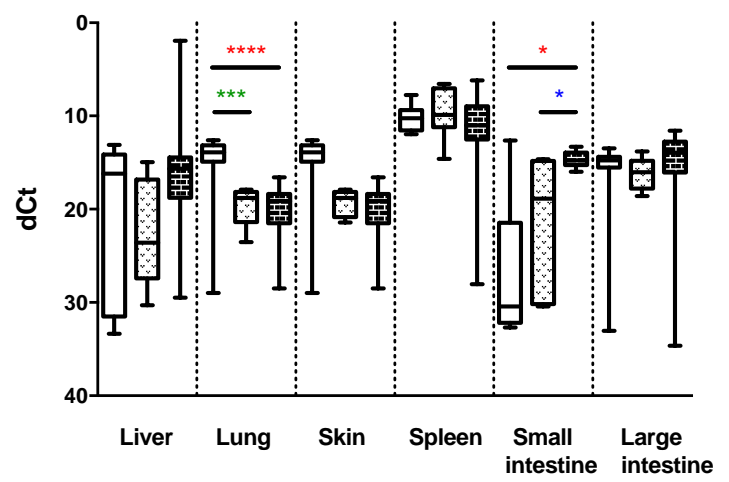

E Tgm2

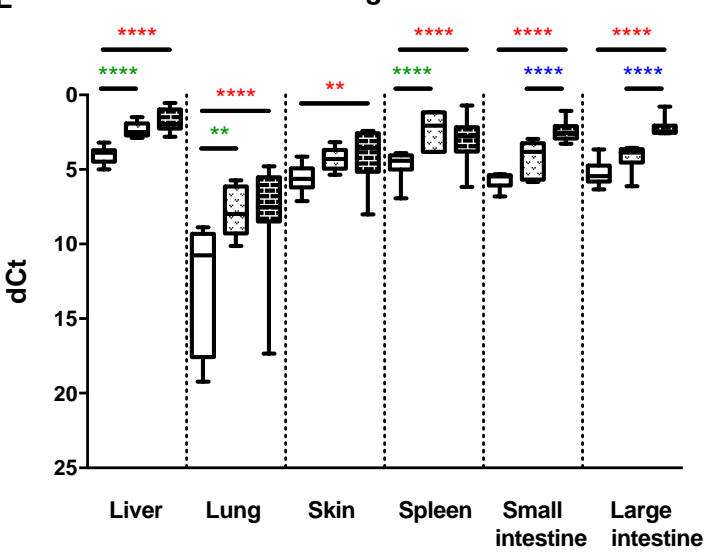

Figure 3.15: mRNA expression of candidate genes regulated by innate immune responses in different tissues in mice.

Relative mRNA expression of candidate genes regulated by innate immune responses, in aGvHD mice ( $\mathrm{n}=$ 17) in comparison with normal healthy controls $(\mathrm{n}=15)$ (red), in aGvHD mice compared to BM control mice $(\mathrm{n}=6)$ (blue) and preconditioning alone by comparing BM control mice to healthy controls (green). The yaxis shows relative expression $(\mathrm{dCt})$, and $\mathrm{x}$ axis shows different mice groups in each tissue type. The $\mathrm{P}$ values were calculated by Mann-Whitney U-test, and were corrected for FDR by Benjamin Hochberg correction. 
$* \mathrm{P}<0.05, * * \mathrm{P}<0.01, * * * \mathrm{P}<0.001, * * * * \mathrm{P}<0.0001$ (significantly regulated genes in aGvHD mice compared to healthy controls)

$* \mathrm{P}<0.05, * * \mathrm{P}<0.01, * * * \mathrm{P}<0.001, * * * * \mathrm{P}<0.0001$ (significantly regulated genes in aGvHD mice compared to $\mathrm{BM}$ controls)

$* \mathrm{P}<0.05, * * \mathrm{P}<0.01, * * * \mathrm{P}<0.001, * * * * \mathrm{P}<0.0001$ (significantly regulated genes in $\mathrm{BM}$ control mice due to preconditioning)

The expression of Galectin-7 (Lgals7) mRNA was significantly reduced in the lung $(0.01 ; \mathrm{p}=$ $0.001)$ in $\mathrm{BM}$ control mice due to preconditioning and in aGvHD mice $(0.01 ; \mathrm{p}=0.0001)$, compared to healthy controls. In contrast, we observed a significant upregulation of Lgals7 in the BM control small intestine $(107.564 ; \mathrm{p}=0.02)$ as well as in the aGvHD small intestine $\left(6.4 \times 10^{2}\right.$; $\mathrm{p}=0.038$ ) compared to healthy controls (Figure 3.15D). LGALS7 is involved in modulating apoptosis and tumor growth (St-Pierre et al., 2012).

Interestingly, Transglutaminase 2 (Tgm2) was significantly upregulated in all the tissues, in aGvHD mice compared to healthy controls, i.e., liver (5.05; $p=0.0001)$, lung $(\mathbf{2 6 . 2 8} ; \mathrm{p}=0.0001)$, skin (3.07; $p=0.002)$, spleen $(3.22 ; p=0.0001)$, small $(9.91 ; p=0.0001)$ and large $(8.76 ; p=$ 0.0001 ) intestines (Figure 3.15E). In the river (3.71; $p=0.0001)$, lung $(\mathbf{3 0 . 8 2} ; p=0.005$ ) and spleen (5.02; $\mathrm{p}=0.0001)$, the increased $\operatorname{Tgm} 2$ expression was in part due to preconditioning in the BM control compared to healthy controls. In addition, Tgm2 was also increased in aGvHD small intestine (3.44; $p=0.0001)$ and large intestine $(\mathbf{3 . 9 4} ; \mathrm{p}=0.0001)$ compared to BM controls. TGM2 is induced by retinoic acid and is involved in apoptosis (Rébé et al., 2009), inflammation and tumor biology (Griffin et al., 2002).

\subsubsection{Rats}

No significant change in gene expression was observed in Clqtnf7, Lgals7 or $\operatorname{Tgm} 2$ whereas a downregulation, similar to that in mice, was observed in the expression of Htral in the lung (0.53; $\mathrm{p}=0.0041$ ) (Figure 3.16B). However, the trend of the regulation pattern of Clqtnf7 in rat aGvHD was similar in the lung, skin and small intestine compared to mice aGvHD (Figure 3.16A). Furthermore, Lglas 7 showed a similar expression pattern in the rat aGvHD in the liver and small intestine (Figure 3.16C), whereas the expression pattern of $\operatorname{Tgm} 2$ showed a trend of upregulation in all tissues rat aGvHD, same as in the mice aGvHD (Figure 3.16D). 


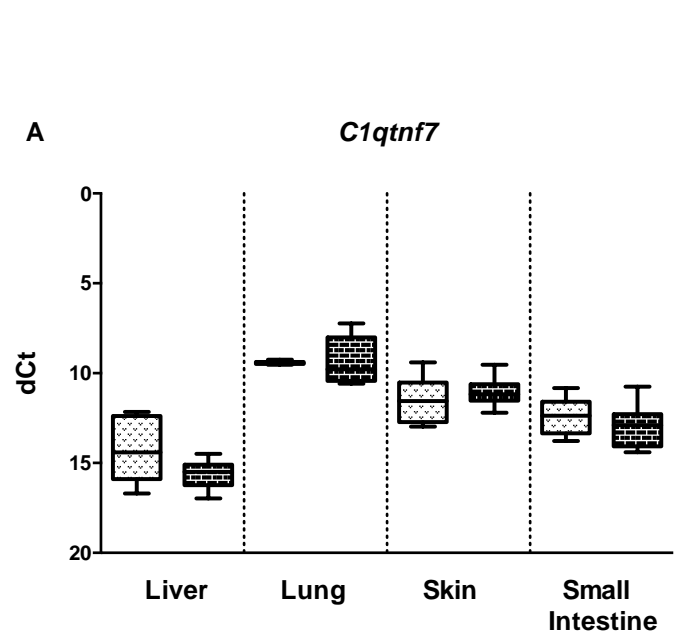

B

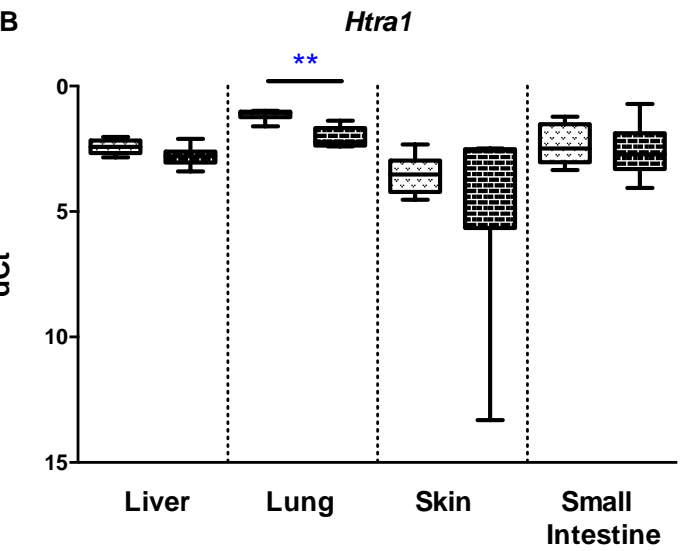

C

Lglas7

D

Tgm2
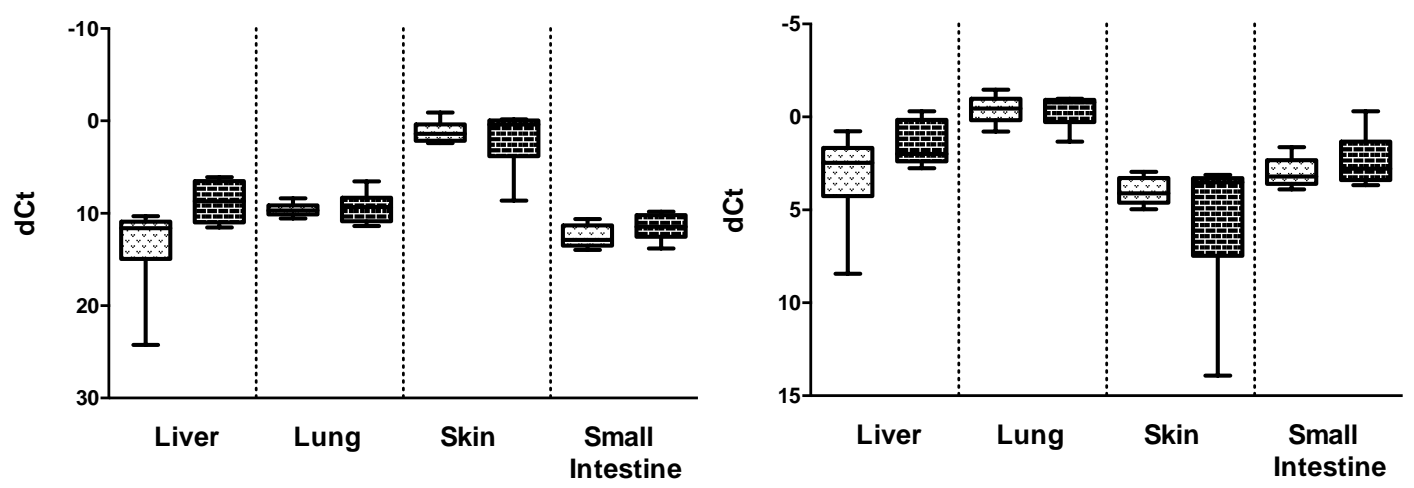

Figure 3.16: mRNA expression of genes regulated by innate immune responses in different tissues in rats.

Relative mRNA expression of genes regulated by innate immune responses in rats with aGvHD, $(n=6)$ on comparison with syngeneic controls $(n=6)$. The $y$-axis shows relative expression $(\mathrm{dCt})$, and $\mathrm{x}$-axis shows control rat and aGvHD expression in different tissues. The $\mathrm{P}$ values were calculated by Mann-Whitney Utest, and were corrected for FDR by Benjamin Hochberg correction.

$* \mathrm{P}<0.05, * * \mathrm{P}<0.01, * * * \mathrm{P}<0.001, * * * * \mathrm{P}<0.0001$

In summary, several candidate genes showed a mixed expression pattern in the different target tissues. However, most of the genes regulated by IFN- $\gamma$ were upregulated significantly in the mice, and showed a similar trend in the rats. Several other genes were also increased in the aGvHD mice and rats, however, we observed a downregulation in genes such as Tap1, Trem2 in the mice, and Htral was reduced in both rat and mice aGvHD. 


\subsubsection{Gene expression of Klrk1 and its ligands in different tissues}

\subsubsection{Mice}

In aGvHD mice compared to healthy controls, an increased expression pattern of Klrkl, encoding NKG2D was observed in all the aGvHD target tissues; liver (2.32; $p=0.001)$, lung (15.55; $p=$ 0.001), skin (7.2; p < 0.0001), small (4.29; $\mathrm{p}<0.0001)$ and large $(3.12 ; \mathrm{p}<0.0001)$ intestines (Figure 3.17A). In both the liver $(\mathbf{2 . 9 7} ; \mathrm{p}=0.002)$ and lung $(\mathbf{7 . 2 7} ; \mathrm{p}=0.023)$, Klrkl mRNA was also increased in BM control mice compare to healthy controls due to preconditioning alone. Moreover, Klrkl was increased in aGvHD skin $(6.42 ; p=0.013)$, small $(4.64 ; p<0.0001)$ and large $(3.67 ; \mathrm{p}<0.0001)$ compared to BM controls.

On the other hand, the expression pattern of Rael showed a strong downregulation in aGvHD liver (0.05; $\mathrm{p}=0.005)$, lung (0.02; $\mathrm{p}=0.004)$, small (0.09; $\mathrm{p}<0.0001)$ and large $(\mathbf{0 . 1} ; \mathrm{p}<0.0001)$ intestines compared to healthy controls. This downregulation was in part, a result of preconditioning in the BM control liver $(\mathbf{0 . 1 0} ; \mathrm{p}=0.006)$, lung $(\mathbf{0 . 0 2} ; \mathrm{p}<0.0001)$, small $(\mathbf{0 . 0 0 1} ; \mathrm{p}$ $<0.0001)$ and large $(\mathbf{0 . 0 5} ; \mathrm{p}=0.011)$ intestines compared to healthy controls. Interestingly, the expression of Rael was increased in the aGvHD small intestine (20.05; $\mathrm{p}=0.048)$ compared to the BM controls (Figure 3.17B). In contrast, H60a showed a more complex expression pattern across the different mouse tissues. In aGvHD liver $(3.7 ; p=0.048)$, skin $(\mathbf{2 . 9 4} ; \mathrm{p}=0.048)$ and large intestine (3.46; $\mathrm{p}=0.048$ ), the expression of $H 60 a$ was significantly increased, in contrast to the lung $(\mathbf{0 . 1 7} ; \mathrm{p}=0.048)$ and spleen $(\mathbf{0 . 8 7} ; \mathrm{p}=0.048)$, where it was downregulated in comparison to healthy controls. However, preconditioning in BM control mice resulted in a significant increase in the expression of $H 60 a$ in the liver $(\mathbf{5 . 2 6} ; \mathrm{p}=0.003)$ but a downregulation in the lung $(\mathbf{0 . 3 0} ; \mathrm{p}=$ $0.036)$ and small intestine $(\mathbf{0 . 3 2} ; \mathrm{p}=0.05)$ (Figure 3.17C).

Ulbp1 encoding MULT-1, showed a clear upregulation across all the tissues during aGvHD. The overall expression in aGvHD mice compared to healthy controls showed a significant increase in the regulation of Ulbpl in the liver (4.46; $\mathrm{p}<0.0001)$, lung (4.82; $\mathrm{p}<0.0001)$ and spleen $(1.87 ; \mathrm{p}$ $=0.012)$ compared to healthy controls, and in aGvHD liver $(\mathbf{2 . 1 4} ; \mathrm{p}<0.0001)$, skin $(\mathbf{1 . 9 9} ; \mathrm{p}=$ 0.044), spleen (1.36; $\mathrm{p}=0.04)$, small intestine $(\mathbf{2 . 3 2} ; \mathrm{p}=0.037)$ and large intestine $(\mathbf{1 . 3 9} ; \mathrm{p}=0.05)$ compared to BM controls. Moreover, the upregulation of Ulbpl was also a result of preconditioning in BM control liver $(\mathbf{2 . 0 8} ; \mathrm{p}=0.008)$ and lung $(\mathbf{6 . 5 3} ; \mathrm{p}=0.008)$ compared to healthy controls (Figure 3.17D). 
A

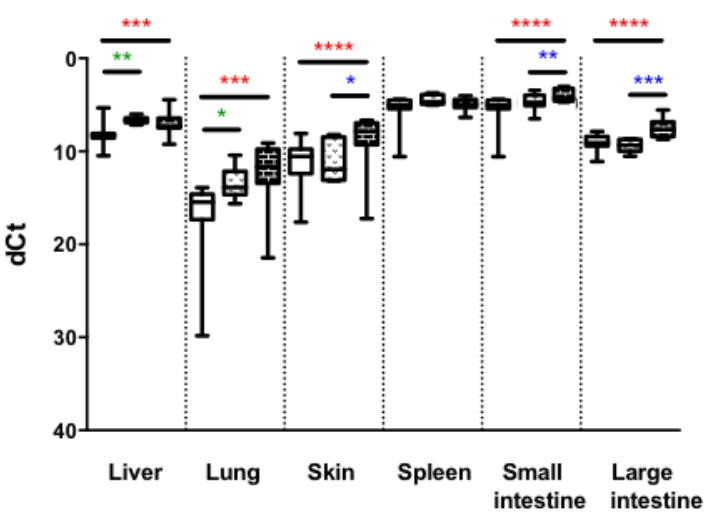

C

H60a

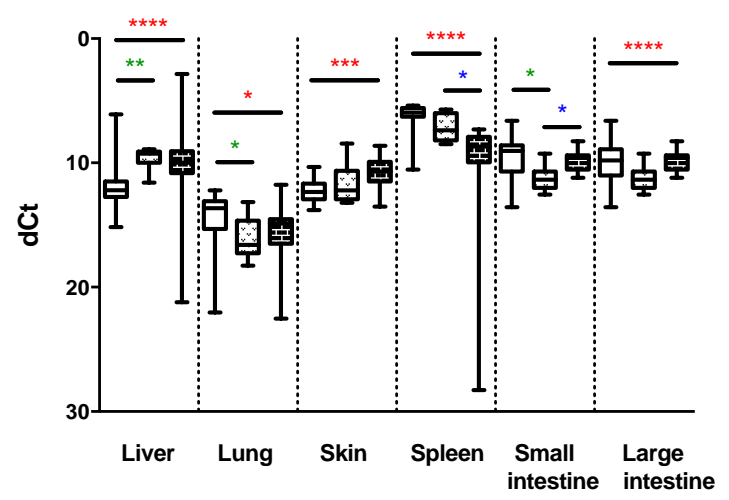

B

Rae1

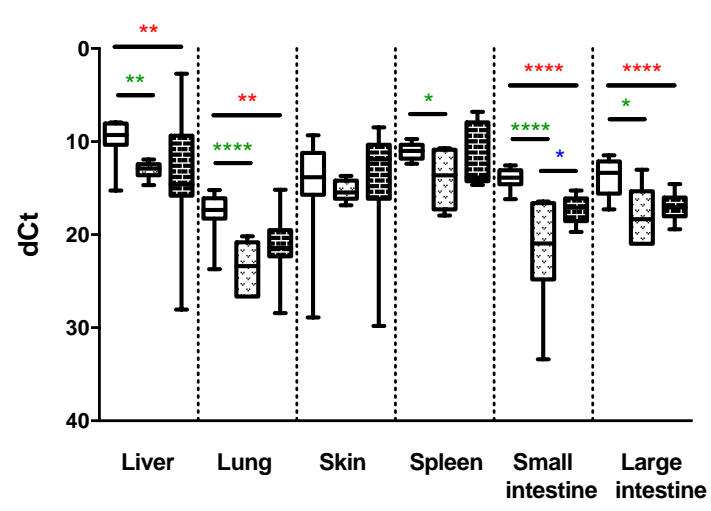

D

Ulbp1
(MULT-1)

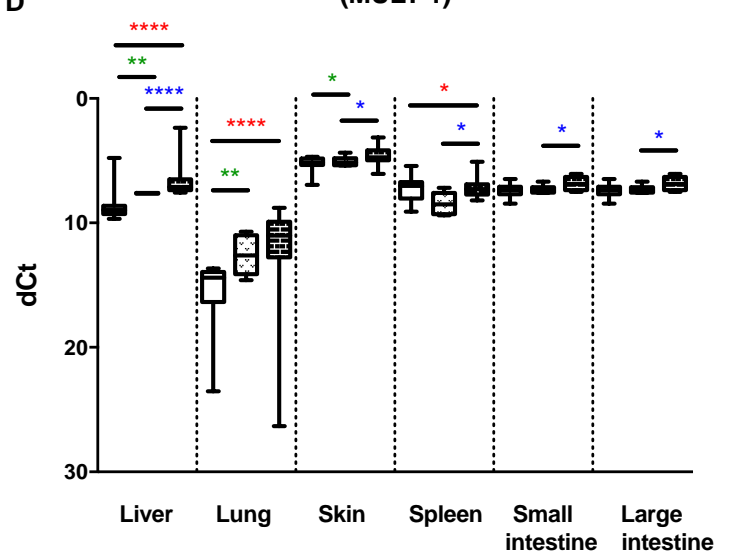

Figure 3.17: mRNA expression of Klrk1 and its ligands in different tissues in mice. Relative mRNA expression of A. Klrk1, B. Rael C. H60 D. Ulbpl, in aGvHD mice (n = 17) in comparison with normal healthy controls $(n=15)(\mathrm{red})$, in aGvHD mice compared to BM control mice $(\mathrm{n}=6)(\mathrm{blue})$ and preconditioning alone by comparing BM control mice to healthy controls (green). The y-axis shows relative expression $(\mathrm{dCt})$, and $\mathrm{x}$ axis shows different mice groups in each tissue type. The $\mathrm{P}$ values were calculated by Mann-Whitney U-test, and were corrected for FDR by Benjamin Hochberg correction.

$* \mathrm{P}<0.05, * * \mathrm{P}<0.01, * * * \mathrm{P}<0.001$, $* * * * \mathrm{P}<0.0001$ (significantly regulated genes in aGvHD mice compared to healthy controls)

$* \mathrm{P}<0.05, * * \mathrm{P}<0.01, * * * \mathrm{P}<0.001$, $* * * * \mathrm{P}<0.0001$ (significantly regulated genes in aGvHD mice compared to BM controls)

$* \mathrm{P}<0.05, * * \mathrm{P}<0.01, * * * \mathrm{P}<0.001, * * * * \mathrm{P}<0.0001$ (significantly regulated genes in $\mathrm{BM}$ control mice due to preconditioning) 


\subsubsection{Rats}

Gene expression of Klrkl was not significantly regulated in any of the tissues in rats with aGvHD. However, a similar trend of increased pattern of Klrkl mRNA expression was observed in the liver and skin, as was observed in mice (Figure 3.18A). Rrlt mRNA was also not regulated in any of the tissues (Figure 3.18C). However, Raell mRNA was upregulated significantly in the liver (185.78; $\mathrm{p}=0.047)$ but downregulated in the skin $(\mathbf{0 . 0 1} ; \mathrm{p}=0.045)($ Figure $3.18 \mathrm{~B})$.

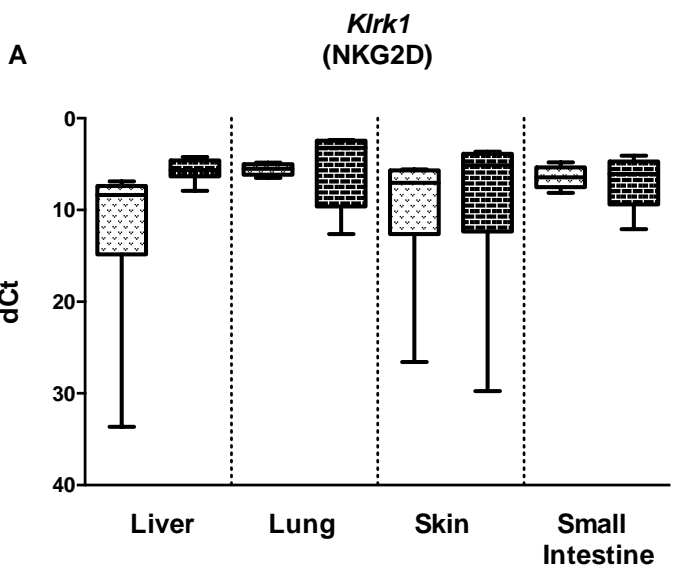

B

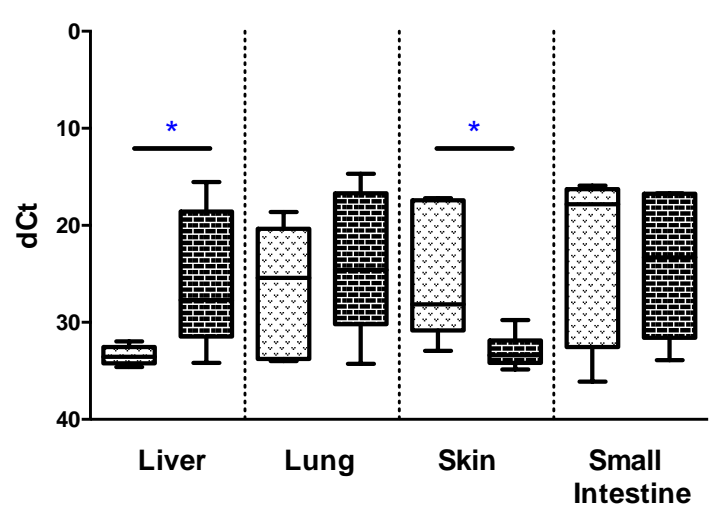

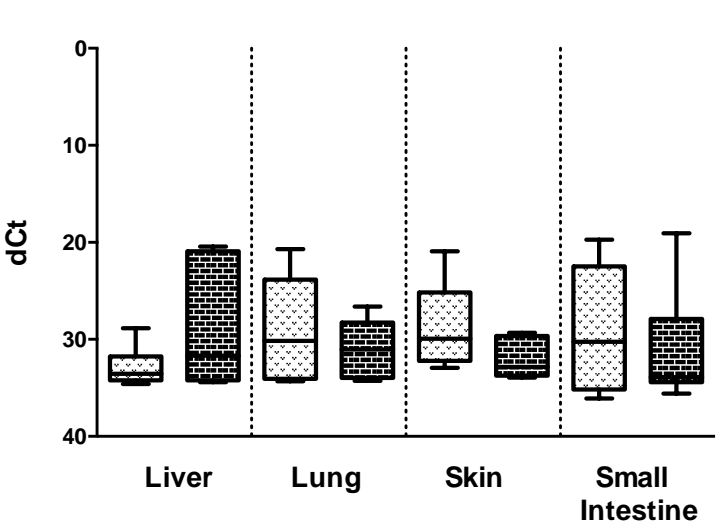

Figure 3.18: mRNA expression of $K l r k 1$ and its ligands in different tissues in rat.

Relative mRNA expression of A. Klrkl and its ligands, B. Raell and C. Rrlt in rats with aGvHD, $(\mathrm{n}=6)$ on comparison with syngeneic controls $(\mathrm{n}=6)$. The $\mathrm{y}$-axis shows relative expression $(\mathrm{dCt})$, and $\mathrm{x}$-axis shows control rat and aGvHD expression in different tissues. The P-values were calculated by Mann-Whitney Utest. $* \mathrm{P}<0.05, * * \mathrm{P}<0.01, * * * \mathrm{P}<0.001, * * * * \mathrm{P}<0.0001$

Overall, the Klrk1 mRNA was upregulated in all the tissues in the mice, but was not significant in the spleen. In the rats, the Klrkl gene regulation was not statistically significant, however it showed a similar trend of upregulation as in the rat. The NKG2D ligands, Rael was significantly decreased in aGvHD mice tissues compared to healthy controls, in contrast to Ulbpl, which was significantly 
upregulated in all aGvHD mice tissues when compared to healthy controls or BM control mice. On the other hand, H60a had a mixed expression pattern in the different mice tissues, however, it was significantly regulated in all the mice aGvHD tissues. In the rat, Raell showed a mixed expression pattern in the different tissues, whereas the expression of Rrlt remained unchanged.

\subsubsection{Gene expression of $C d 226$ and its ligands in different tissues}

\subsubsection{Mice}

The expression of $C d 226$ encoding DNAM-1 was also significantly upregulated in all the aGvHD mice tissues, liver $(1 ; p=0.003)$, lung (4.25; $p=0.006)$, skin (10.54; $p<0.0001)$, small (10.66; $p$ $<0.0001)$ and large $(4.89 ; \mathrm{p}<0.0001)$ intestines compared to healthy controls. We also observed a significant increase in the expression of $C d 226$ between aGvHD liver (1.44; $\mathrm{p}=0.016)$, skin (9.16; $\mathrm{p}=0.003)$, spleen $(\mathbf{1 . 6 1} ; \mathrm{p}=0.027)$, small intestine $(\mathbf{8 . 5 2} ; \mathrm{p}<0.0001)$ and large intestine $(\mathbf{1 0 . 8} ; \mathrm{p}$ $<0.0001$ ) compared to BM controls (Figure 3.19A). Similarly, the DNAM-1 ligand, Pvrl2, encoding CD112, was also significantly upregulated in aGvHD liver (2.23; p $<0.0001)$, lung (2.43; $\mathrm{p}=0.03)$, spleen $(\mathbf{2 . 3 9} ; \mathrm{p}=0.001)$, small (2.83; $\mathrm{p}<0.0001)$ and large $(\mathbf{1 . 9 2} ; \mathrm{p}=0.003)$ intestines (Figure 3.19B). The increased expression of Pvrl2 was in part due to preconditioning in the BM control liver $(\mathbf{1 . 7 9} ; \mathrm{p}=0.001)$, lung $(\mathbf{3 . 8 4} ; \mathrm{p}=0.036)$ and spleen $(\mathbf{4 . 6 5} ; \mathrm{p}<0.0001)$ compared to healthy controls. Furthermore, in the small intestine, we also observed a significant increase in the expression of Pvrl2 between the aGvHD mice and BM control mice $(1.82 ; \mathrm{p}=$ 0.048). The expression of the other ligand $P v r$ encoding CD155, was also strongly upregulated in the aGvHD skin $(\mathbf{2 . 5 6} ; \mathrm{p}=0.048)$, small $(\mathbf{3 . 3 8} ; \mathrm{p}=0.048)$ and large intestine $(\mathbf{1 . 4 1} ; \mathrm{p}=0.048)$ compared to healthy controls, as well as in the skin $(\mathbf{8 . 3 2} ; \mathrm{p}=0.006)$ and large intestine $(\mathbf{5 . 8 9} ; \mathrm{p}=$ 0.001) compared to BM controls (Figure 3.19C). 

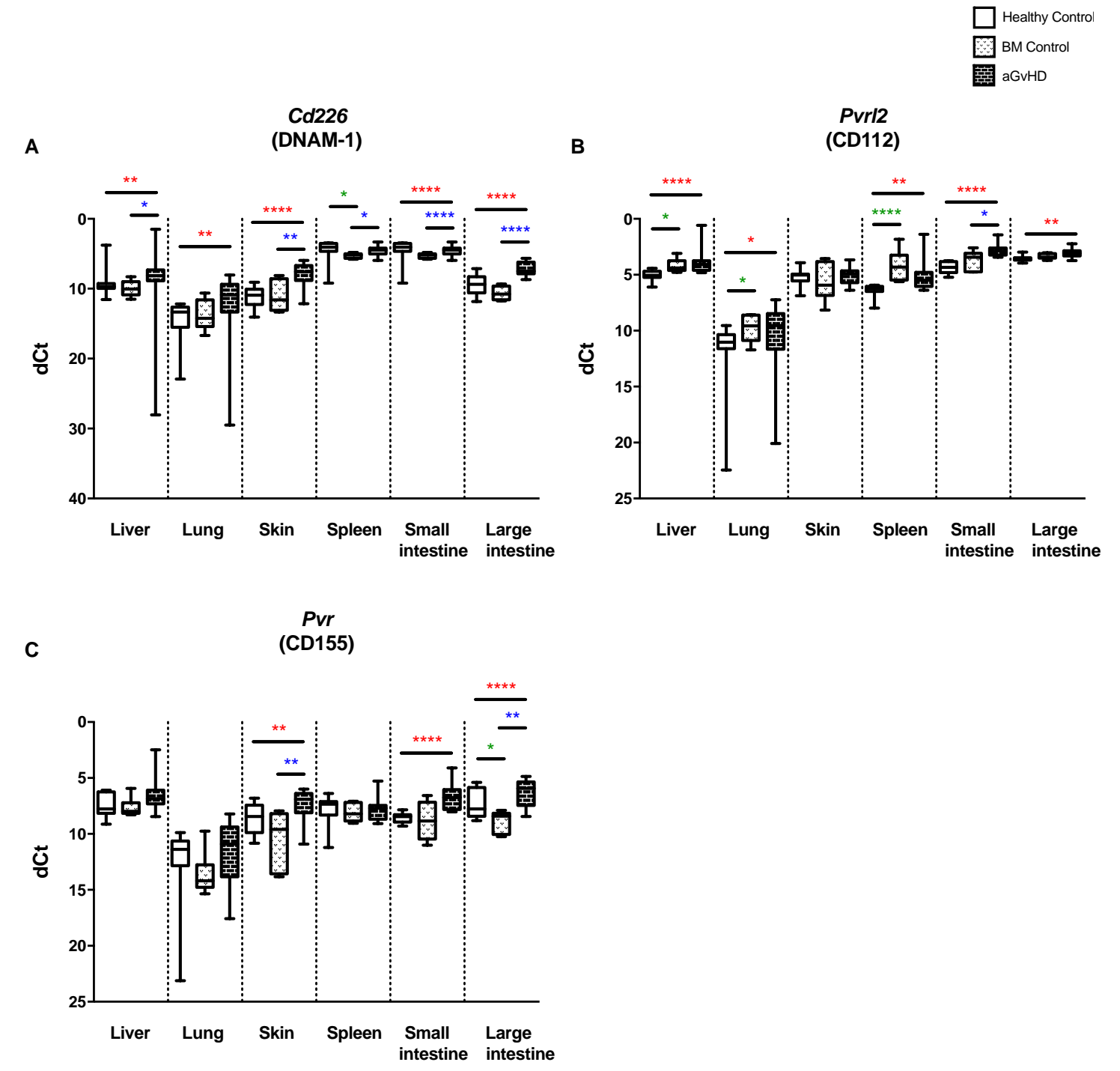

Figure 3.19: mRNA expression of $C d 226$ and its ligands in different tissues in mice.

Relative mRNA expression of A. Cd226, and its ligands B. Pvrl2 (CD112) and C. Pvr (CD155), in aGvHD mice $(\mathrm{n}=17)$ in comparison with normal healthy controls $(\mathrm{n}=15)(\mathrm{red})$, in aGvHD mice compared to BM control mice $(\mathrm{n}=6)$ (blue) and preconditioning alone by comparing BM control mice to healthy controls (green). The $\mathrm{y}$-axis shows relative expression $(\mathrm{dCt}$ ), and $\mathrm{x}$ axis shows different mice groups in each tissue type. The P values were calculated by Mann-Whitney U-test, and were corrected for FDR by Benjamin Hochberg correction.

$* \mathrm{P}<0.05, * * \mathrm{P}<0.01, * * * \mathrm{P}<0.001$, **** $\mathrm{P}<0.0001$ (significantly regulated genes in aGvHD mice compared to healthy controls)

$* \mathrm{P}<0.05, * * \mathrm{P}<0.01, * * * \mathrm{P}<0.001, * * * * \mathrm{P}<0.0001$ (significantly regulated genes in aGvHD mice compared to BM controls)

$* \mathrm{P}<0.05, * * \mathrm{P}<0.01, * * * \mathrm{P}<0.001, * * * * \mathrm{P}<0.0001$ (significantly regulated genes in $\mathrm{BM}$ control mice due to preconditioning) 


\subsubsection{Rats}

Cd226 mRNA was not significantly regulated in any of the tissues, however a trend of increased expression of $C d 226$ was observed in the liver and skin, similar to that in mice (Figure 3.20A). Pvrl2 was significantly increased in the small intestine $(\mathbf{2 . 8 7} ; \mathrm{p}=0.0077)$, however a trend of upregulation was observed in the liver, lung and skin as well (Figure 3.20B). No change in gene expression was observed in $P v r$, in any of the rat aGvHD tissues, although a trend of upregulation was observed in all tissues similar to that in mice, except the liver (Figure 3.20C).

A

\section{Dnam-1}

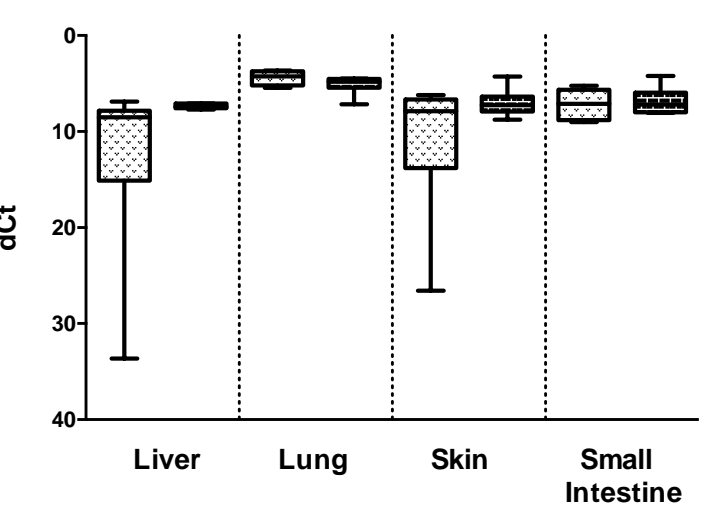

B

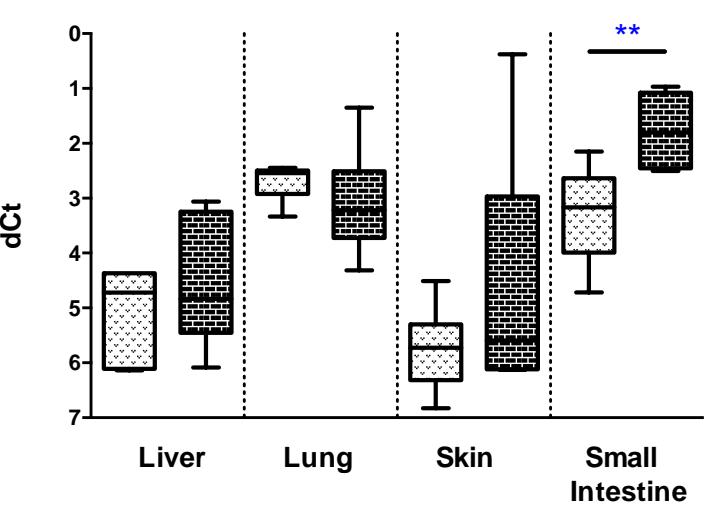

C
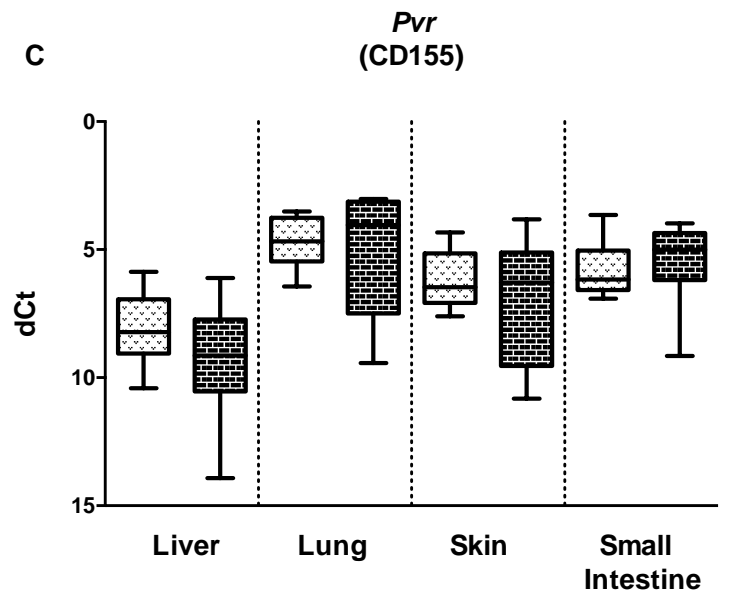

Figure 3.20: mRNA expression of $\mathrm{Cd} 226$ and its ligands in different tissues in rats.

Relative mRNA expression of A. Dnam- 1 and its ligands, B. Pvrl2 and C. Pvr in rats with aGvHD, $(\mathrm{n}=6)$ on comparison with syngeneic controls $(n=6)$. The $y$-axis shows relative expression $(\mathrm{dCt})$, and $\mathrm{x}$-axis shows control rat and aGvHD expression in different tissues. The $\mathrm{P}$ values were calculated by Mann-Whitney $\mathrm{U}-$ test, and were corrected for FDR by Benjamin Hochberg correction.

$* \mathrm{P}<0.05, * * \mathrm{P}<0.01, * * * \mathrm{P}<0.001, * * * * \mathrm{P}<0.0001$

In summary, we observed an upregulation of $C d 226$ and its ligands Pvrl2 and $P v r$ in the different tissues. $C d 226$ was upregulated in all the aGvHD tissues during aGvHD, whereas Pvrl2 was 
significantly upregulated in all the tissues except the skin, and $P v r$ was upregulated in three tissues. Similarly, in the rat aGvHD as well $C d 226$ and its ligands showed a trend of upregulation in all the tissues, however Pvrl2 was only significant in the small intestine.

\subsection{T cell infiltration in the small and large intestines during aGvHD}

On IHC staining, we observed infiltrating cells expressing CD3 in the small (Figure 3.21A) and large intestine (Figure 3.21B) biopsies during aGvHD. In the large intestine, we also observed substantial number of infiltrating $\mathrm{T}$ cells in the $\mathrm{BM}$ control, as a result of preconditioning. Moreover, the infiltrating T cells could explain the upregulation of several of our focus genes that are associated with $\mathrm{T}$ cell activation and cytokines and chemokines that are induced as a result of activation of T cells.

In addition to $\mathrm{T}$ cell infiltration, we observed loss of crypts, and necrosis, both symptoms of severe aGvHD. The histological observations of the intestines are consistent with severe aGvHD in the intestines, which could also explain a more predominant expression of several genes in the intestines.

A

healthy control

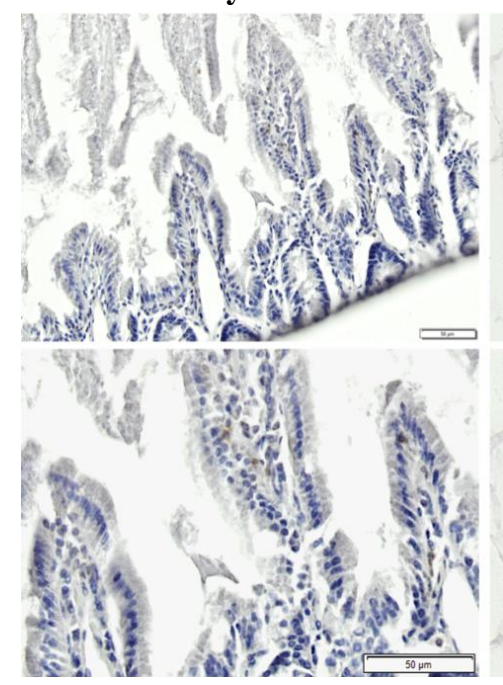

bone marrow control
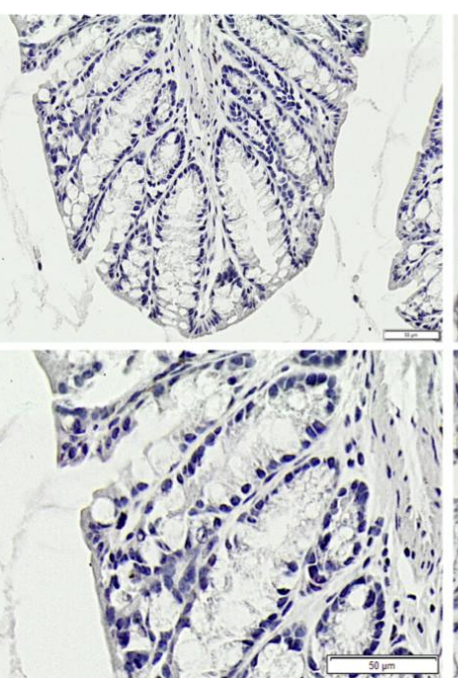

aGvHD

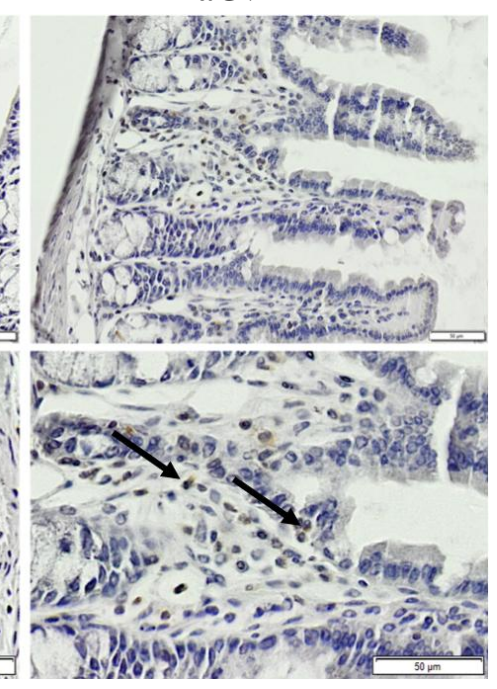




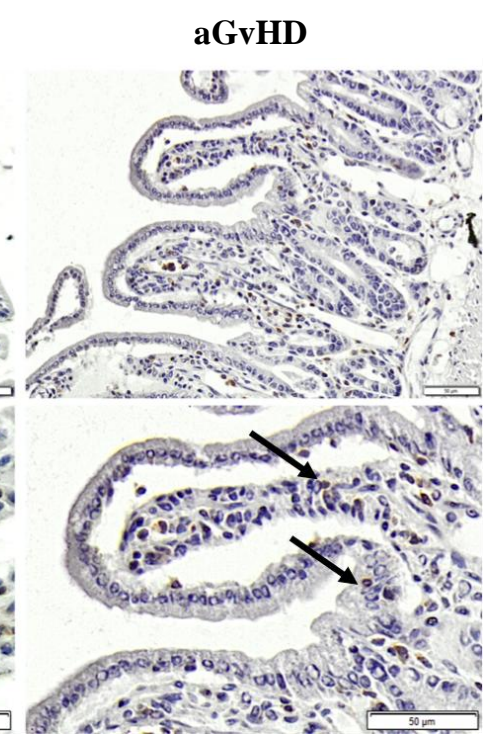

Figure 3.21: CD3 staining of small and large intestines in mice.

A. CD3 immunohistological staining of healthy, bone marrow control and aGvHD small intestine in mice. B. CD3 immunohistological staining of healthy, bone marrow control and aGvHD small intestine in mice. The arrows show CD3 stained infiltrating cells in the tissues.

\subsection{Human aGvHD GI biopsies}

We studied gene expression of all the selected genes in human gastrointestinal biopsies $(n=186)$.

\subsubsection{Patient Cohort}

The patient biopsies from different parts of the gastrointestinal tract (GI) were obtained from University Klinik Regensburg from Prof. Ernst Holler's group. Patients underwent transplantation from Match 2009 till October 2013.

\section{Table 3.1: Patient characteristics}

\section{Characteristics}

Values

\section{Recipients ( $\mathbf{n = 1 8 6 )}$}

Median age, $y$

Younger than $20 \mathrm{y}, \mathrm{n}(\%)$

20 to $40 \mathrm{y}, \mathrm{n}(\%)$

Older than $40 \mathrm{y}, \mathrm{n}(\%)$

Male, n (\%)

Female, n (\%) 68 (36.6) 


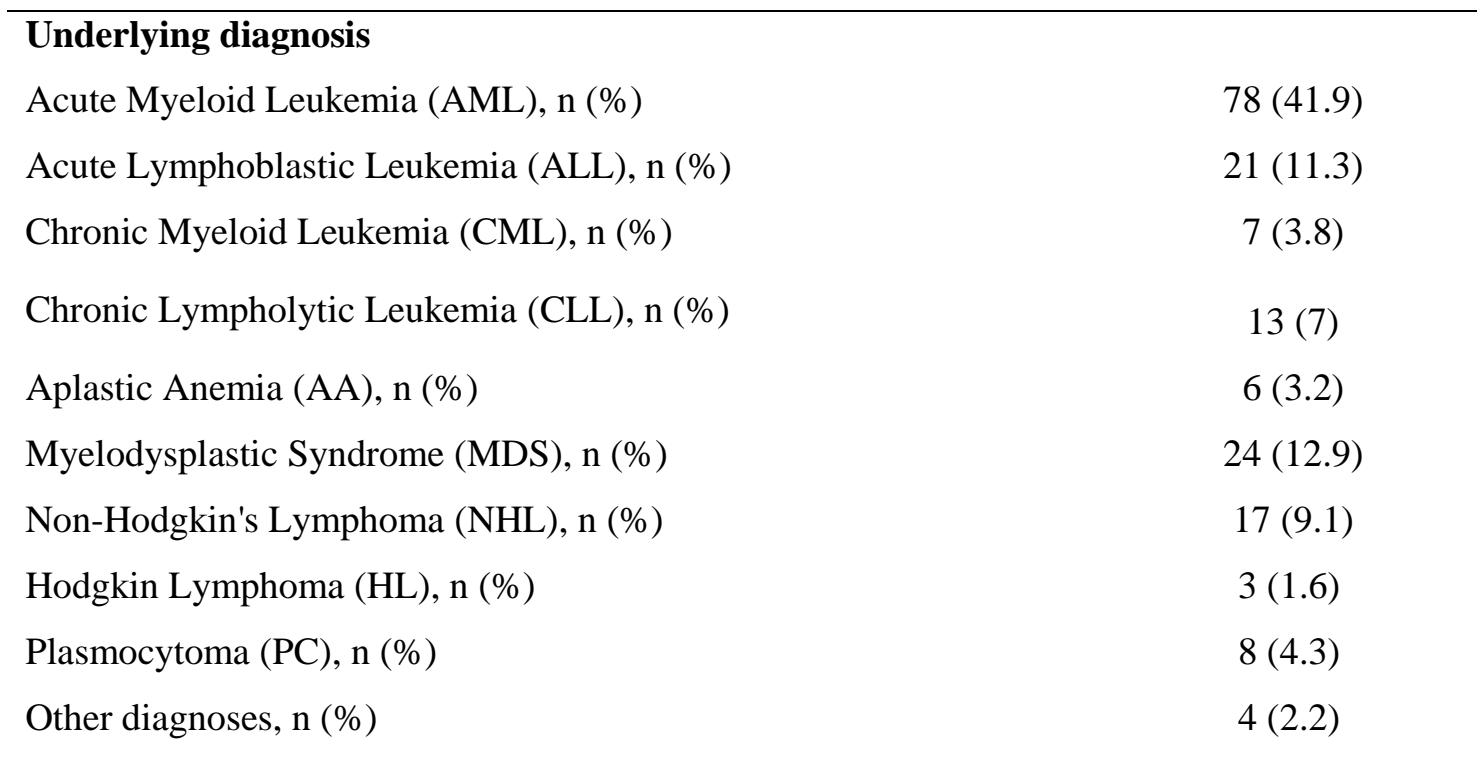

\section{Disease status for malignant disorders}

Early, n (\%)

Intermediate, $\mathrm{n}(\%)$

$64(34.4)$

Advanced, n (\%)

68 (36.6)

$\mathrm{ND}, \mathrm{n}(\%)$

$8(4.3)$

\section{Donors ( $(n=186)$}

Median age, $y$

Younger than $20 \mathrm{y}, \mathrm{n}(\%)$

20 to $40 \mathrm{y}, \mathrm{n}(\%)$

Older than $40 \mathrm{y}, \mathrm{n}(\%)$

Male, n (\%) $139(74.7)$

Female, n (\%) 47 (25.3)

CMV-donor to $\mathrm{CMV}+$ recipient, $\mathrm{n}(\%)$ 36 (19.4)

$\mathrm{CMV}+$ donor to $\mathrm{CMV}$ - recipient, $\mathrm{n}(\%)$ $19(10.2)$

Female donor to male recipient, $\mathrm{n}(\%)$ 13 (16.7)

HLA-matched unrelated donor, n (\%) $127(68.3)$

Related donor, n (\%) $59(31.7)$

\section{Transplantation}

Source of stem cells

Peripheral blood, n (\%)

$164(88.2)$

Bone marrow, n (\%)

Cord blood, n (\%)

Conditioning

Standard, n (\%) 
$\mathrm{ND}, \mathrm{n}(\%)$

Treatment

Steroid $>20 \mathrm{mg}, \mathrm{n}(\%)$

\section{Outcome}

No GvHD, n (\%)

Grade I to II, n (\%)

Grade III to IV n (\%)

$\mathrm{ND}, \mathrm{n}(\%)$

Transplant related mortality, n (\%)

75 (40.3)

\subsubsection{Gene expression analysis during GI aGvHD in all patients}

We designed a step-wise regression model on $\mathrm{R}$, to select covariates that affect the expression of the selected genes listed in Table 3.2. The regulation of genes was assessed for a number of variables such as GvHD incidence, TRM using a two-sample t-test for the multivariate analyses. Only the statistically significant $p$-values $(p<0.05)$ for expression of genes have been shown and regulation of genes that are near significance $(p<0.15)$. The size of the relative difference to the variation of the sample data is shown by t-values, where a t-value $>0$ shows an upregulation of the gene and a t-value $>0$ shows a downregulation of the gene.

Table 3.2: Genes selected for gene expression study in human GI aGvHD.

\begin{tabular}{|c|c|c|c|}
\hline \multirow{2}{*}{$\begin{array}{l}\text { Genes } \\
I L 2\end{array}$} & \multirow[t]{2}{*}{ Selected Covariates $(p<0.05)$} & \multicolumn{2}{|c|}{ Potential Covariates $(p>0.05)$} \\
\hline & & Type of conditioning & Steroid treatment \\
\hline IL4 & & Stem cell source & EBMT risk score \\
\hline$I L 4 R$ & & CMV risk & \\
\hline IL5 & & Steroid treatment & \\
\hline IL13 & & CMV risk & Stem cell source \\
\hline IL33 & Donor age & EBMT risk score & \\
\hline IL6 & Type of conditioning & Days after transplant & CMV risk \\
\hline IL15 & Stem cell source & Days after transplant & \\
\hline ILIRI & $\begin{array}{l}\text { Type of conditioning } \\
\text { Stem cell source } \\
\text { Blood group }\end{array}$ & & \\
\hline ILIR2 & & Days after transplant & Blood group \\
\hline$T G F B 1$ & & Donor age & \\
\hline TGFBRI & & Days after transplant & Stem cell source \\
\hline
\end{tabular}




\begin{tabular}{|c|c|c|c|}
\hline \multicolumn{2}{|l|}{$T G F B 2$} & \multicolumn{2}{|l|}{ CMV risk } \\
\hline$I L 2 R A$ & Stem cell source & Type of conditioning & \\
\hline CCL4 & & EBMT risk score & Stem cell source \\
\hline CCL5 & & Steroid treatment & Donor age \\
\hline CCR1 & Stem cell source & & \\
\hline CCR4 & & Stem cell source & \\
\hline CCR5 & & CMV risk & $\begin{array}{l}\text { Type of } \\
\text { conditioning }\end{array}$ \\
\hline CX3CR1 & Steroid treatment & & \\
\hline CXCL10 & & Steroid treatment & \\
\hline CXCL11 & & Days after transplant & \\
\hline CXCL16 & & Steroid treatment & \\
\hline CXCL8 & & Steroid treatment & \\
\hline \multicolumn{4}{|l|}{ CXCL9 } \\
\hline \multicolumn{4}{|l|}{ CXCR3 } \\
\hline CXCR4 & & Steroid treatment & \\
\hline ENPP1 & & Type of conditioning & \\
\hline FCER1G & & Steroid treatment & \\
\hline \multicolumn{4}{|l|}{ FCGR3 } \\
\hline ICAMI & Stem cell source & & \\
\hline VCAM1 & Steroid treatment & & \\
\hline$A N P 32 A$ & CMV risk & Type of conditioning & \\
\hline BMP1RA & & EBMT risk score & CMV risk \\
\hline CIQTNF7 & & Steroid treatment & \\
\hline$C A R D 11$ & & EBMT risk score & Donor age \\
\hline HCLS1 & & Steroid treatment & Donor age \\
\hline HTRA1 & & Stem cell source & Blood group \\
\hline LGALS7 & Steroid treatment & Days after transplant & EBMT risk score \\
\hline LILRA5 & EBMT risk score & & \\
\hline LST1 & & CMV risk & \\
\hline MSRI & & Stem cell source & $\begin{array}{l}\text { Days after } \\
\text { transplant }\end{array}$ \\
\hline PIKЗАP1 & & Steroid treatment & Stem cell source \\
\hline PSTPIP1 & & Donor age & Stem cell source \\
\hline PTGER2 & & Type of conditioning & EBMT risk score \\
\hline \multirow[t]{2}{*}{ PTPN7 } & EBMT risk score & & \\
\hline & Blood group & & \\
\hline \multirow[t]{2}{*}{ TAP1 } & Type of conditioning & & \\
\hline & Stem cell source & & \\
\hline$T G M 2$ & Type of conditioning & Blood group & \\
\hline TREM2 & & Steroid treatment & \\
\hline$U B D$ & & Donor age & \\
\hline KLRK1 & EBMT risk score & Days after transplant & Blood group \\
\hline$M I C A$ & EBMT risk score & CMV risk & \\
\hline MICB & & Stem cell source & \\
\hline$U L B P 1$ & CMV risk & Blood group & \\
\hline \multicolumn{4}{|l|}{$U L B P 2$} \\
\hline ULBP3 & & Days after transplant & \\
\hline CD226 & Type of conditioning & Days after transplant & Steroid treatment \\
\hline$P V R$ & Steroid treatment & Type of conditioning & \\
\hline
\end{tabular}


Listed are genes and selected covariates that affect their expression during GI aGvHD in human biopsies.

Steroid treatment - patients that received steroid vs. patients that received $<20 \mathrm{mg} / \mathrm{Kg}$ or no steroids; EBMT risk score - described in section 2.2.5.3, in Table 2.12;

$\mathrm{CMV}$ risk - CMV- donor to CMV+ recipient (score $=2$ ), $\mathrm{CMV}+$ donor to $\mathrm{CMV}$ - recipient (score=1), both donor and recipient with same CMV status (score $=0$ ).

The selected covariates are statistically significant covariates that affect the expression of the specified gene. Based on the model specified, we calculated the gene expression for different specified variables. Interestingly, the expression of several genes from the previously identified candidate genes and Klrkl and its ligands were affected by EBMT score. Other covariates that were found to be important were the type of conditioning, CMV risk, steroid treatment, type of conditioning regimen and source of stem cell. The days after transplant could be a potential covariate affecting the expression of several genes, however it was not selected as one of the more important ones. Our gene expression data has been analyzed with a multivariate analysis to compensate for all the selected covariates.

\subsubsection{Effect of clinical GI GvHD grade on gene expression patterns of the selected genes}

The patients were graded in the clinic based on symptoms exhibited by the patients such as rash on skin, blood in stool, diarrhea, etc. Here, we analyzed the gene expression in two patient groups, those that did not exhibit any clinical symptoms of aGvHD (grade 0) and patients with clinical aGvHD symptoms (grade 1 to 4). The gene expression was assessed based on the model described in section 2.2.5.2. The significant $(\mathrm{p}<0.05)$ genes and nearly significant genes $(\mathrm{p}<0.15)$ that show a trend of regulation, are listed in Table 3.3 . 


\begin{tabular}{|c|c|c|}
\hline Genes & t-value & p-value $(<0.05)$ \\
\hline HCLS1 & 4.101 & 0.00008 \\
\hline IL2 & 3.04 & 0.002 \\
\hline CCR5 & 3.007 & 0.003 \\
\hline FCGR3 & 2.886 & 0.004 \\
\hline CXCR3 & 2.815 & 0.005 \\
\hline CCR4 & 2.789 & 0.006 \\
\hline TGFB1 & 2.672 & 0.008 \\
\hline CCR1 & 2.652 & 0.009 \\
\hline IL6 & 2.496 & 0.014 \\
\hline CIQTNF7 & 2.44 & 0.014 \\
\hline CXCR4 & 2.374 & 0.016 \\
\hline CCL4 & 2.322 & 0.019 \\
\hline VCAM1 & 2.280 & 0.022 \\
\hline FCERIG & 2.277 & 0.024 \\
\hline$C A R D 11$ & 2.225 & 0.028 \\
\hline PIK3API & 2.1 & 0.038 \\
\hline \multirow[t]{2}{*}{ LST1 } & 2.055 & 0.042 \\
\hline & & $\begin{array}{c}\text { p-value } \\
(<0.15 ; \text { n.s. })\end{array}$ \\
\hline PTGER2 & 1.925 & 0.057 \\
\hline$I L 2 R A$ & 1.908 & 0.059 \\
\hline$I L 1 R I$ & -1.844 & 0.062 \\
\hline HTRAl & -1.836 & 0.076 \\
\hline MSRl & -1.786 & 0.091 \\
\hline PSTPIP1 & 1.55 & 0.124 \\
\hline$M I C B$ & 1.503 & 0.135 \\
\hline PTPN7 & 1.457 & 0.148 \\
\hline
\end{tabular}

Table 3.3: Genes regulated in severe clinical aGvHD (grade 1-4) versus no aGvHD (grade 0) in patients. Genes significantly regulated $(\mathrm{p}<0.05)$ based on clinical aGvHD in human GI biopsies. Additionally, genes that show a trend of significance $(\mathrm{p}<0.15 ; \mathrm{n}$.s. $)$ are listed. $t$-values represent the size of the relative difference to variation of the sample data; $t>0$ shows upregulation and $\mathrm{t}<0$ shows downregulation.

$32.8 \%$ of the patients did not develop clinical aGvHD symptoms, whereas $46.7 \%$ of the patients exhibited grade 1-4 aGvHD. Several genes were significantly upregulated in clinical GI aGvHD compared to patients without aGvHD, including the adhesion molecule, VCAM1 and the cytokines $I L 2$ and IL6. However, we observed a more significant pattern in the expression of chemokines receptors, CCR1, CCR4, CCR5, CXCR3 and CXCR4 and the chemokine ligand CCL4.

It could be that the clinical symptoms are associated with a more dominant tissue-mediated chemokine response, due to tissue injury in the gut, in addition to infiltrating immune cells. In addition several genes associated with $\mathrm{B}$ and $\mathrm{T}$ cell activation, HCLS1, CARD11, PIK3AP1, LST1, FCERIG and FCGR3 were significantly increased, suggesting an infiltration of $\mathrm{B}$ and $\mathrm{T}$ cells in the gut and an activation of alloreactive cells during aGvHD. Interestingly, TGFBl was increased significantly. It has been shown previously that Treg expression is induced in the gut in the presence 
of TGF- $\beta$ (Cobbold et al., 2004). We also found an increase IL2RA (n.s) in the intestinal biopsies, suggesting the expansion of iTregs during aGvHD in the gut.

\subsubsection{Effect of histological aGvHD score on gene expression patterns of the selected genes}

Clinical scoring of patients that experience GI GvHD is not absolutely reliable for determining the extent of aGvHD in the gut, since the clinical scoring involves scoring of other organs such as rash on the skin, and other immeasurable symptoms such as blood in stool, vomiting and diarrhea. Therefore, the biopsies are further graded histologically in the clinic, from grade 0 to 4 , representing a more accurate extent of aGvHD in the gut. It is important to note, therefore that the clinical and histological scoring defers greatly $\left(\mathrm{p}=0.959, \mathrm{R}^{2}=0.004\right)$, however, both scoring systems are equally important. The gene expression was assessed based on the model described in section 3.5.1. Genes regulated during histological aGvHD are listed in Tables 3.4 and 3.5.

\subsection{Histological aGvHD grades 1-4 versus no aGvHD}

The genes regulated between histological aGvHD grades 1-4 versus no aGvHD (grade 0) were assessed.

\begin{tabular}{ccc}
\hline & t-value & p-value $(<\mathbf{0 . 0 5})$ \\
\hline IL2RA & -2.63 & $\mathbf{0 . 0 0 9}$ \\
\hline & & $\begin{array}{c}\text { p-value } \\
(<\mathbf{0 . 1 5} \text {; n.s. })\end{array}$ \\
\hline CCL4 & -1.977 & 0.051 \\
CXCR4 & -1.957 & 0.053 \\
CARD11 & 1.914 & 0.059 \\
FCGR3 & -1.745 & 0.084 \\
ANP32A & 1.635 & 0.105 \\
PSTPIP1 & -1.63 & 0.106 \\
CCR4 & -1.629 & 0.107 \\
LGALS7 & 1.5 & 0.137 \\
\hline
\end{tabular}

Table 3.4: Genes regulated in histological GI aGvHD (grade 1-4) versus no aGvHD in patients. Genes significantly regulated $(\mathrm{p}<$ 0.05 ) based on histological aGvHD in human GI biopsies. Additionally, genes that show a trend of significance $(\mathrm{p}<0.15$; n.s. $)$ are listed. $\mathrm{t}$ values represent the size of the relative difference to variation of the sample data; $t$ $>0$ shows upregulation and $\mathrm{t}<0$ shows downregulation.

The gene expression during aGvHD (histologically scored) was biased towards genes regulated by $\mathrm{T}$ and B cells. A significant downregulation in the IL2RA (CD25) was observed. CD25 is present on a number of immune cells, including Tregs. Moreover, we observed a downregulation of other genes 
associated with T and B cells, CCL4, CCR4, PSTPIP1 and CARD11, whereas genes associated with innate immune responses, ANP32A and LGALS7 were increased.

\subsection{Histological aGvHD grades 2-4 versus no or low aGvHD (grades 0-1)}

The genes regulated between histological aGvHD grades 2-4 versus no or low aGvHD (grade 0-1) were assessed.

\begin{tabular}{ccc}
\hline Genes & t-value & p-value $(<\mathbf{0 . 0 5})$ \\
\hline CXCL8 & -3.144 & $\mathbf{0 . 0 0 2}$ \\
CCL4 & -3.047 & $\mathbf{0 . 0 0 3}$ \\
CX3CR1 & 2.453 & $\mathbf{0 . 0 1 6}$ \\
CXCL16 & 2.275 & $\mathbf{0 . 0 2 5}$ \\
ULBP3 & 2.111 & $\mathbf{0 . 0 3 7}$ \\
ENPP1 & -1.985 & $\mathbf{0 . 0 5}$ \\
LGALS7 & 1.957 & $\mathbf{0 . 0 5}$ \\
\hline & & $\mathbf{p}-$ value \\
& -1.862 & 0.066 \\
\hline$P S T P I P 1$ & -1.76 & 0.082 \\
CXCL9 & -1.709 & 0.091 \\
$I L 2 R A$ & -1.677 & 0.097 \\
TAP1 & -1.662 & 0.1 \\
\hline CI $Q T N F 7$ & &
\end{tabular}

Table 3.5: Genes regulated in severe histological GI aGvHD (grade 2-4) versus no or low aGvHD (grade $0-1$ ) in patients. Genes significantly regulated $(\mathrm{p}<0.05)$ based on histological aGvHD in human GI biopsies. Additionally, genes that show a trend of significance $(\mathrm{p}<0.15$; n.s. $)$ are listed. $\mathrm{t}$-values represent the size of the relative difference to variation of the sample data; $t>0$ shows upregulation and $\mathrm{t}<0$ shows downregulation.

Severe aGvHD was associated with a decrease in the gene expression of the chemokines CXCL8 and CCL4 were significantly decreased, but an increase in the expression of chemokine receptor, CX3CR1 and CXCL16 mRNA were significantly increased. Moreover, the NKG2D ligand, ULBP3 was significantly upregulated.

Taken together, we observed that overall aGvHD (grades 1-4) was associated with an increase in genes associated with $\mathrm{B}$ and $\mathrm{T}$ cell activation, whereas severe aGvHD compared to a low aGvHD grade represents a gene regulation pattern associated with innate immune responses, possibly as a result of tissue injury. 


\subsubsection{Effect of gene regulation on transplant related mortality (TRM) in HSCT patients}

The effect of gene regulation on transplant related mortality was studied. We compared patients who died due to transplant related causes to patients who were still alive or died due to unrelated causes such as relapse (Table 3.6). Since $40.3 \%$ of the patients died due transplant related causes, it is possible that they exhibit a different pattern in the expression of certain genes that could be associated with risk of death.

\begin{tabular}{lcc}
\hline \multicolumn{1}{c}{ Genes } & t-value & p-value $(<\mathbf{0 . 0 5})$ \\
\hline CX3CR1 & 1.998 & $\mathbf{0 . 0 4 8}$ \\
\hline & & p-value $(<\mathbf{0 . 1 5})$ \\
\hline PVRL2 & 1.844 & 0.068 \\
CXCL10 & -1.798 & 0.075 \\
CXCL8 & 1.716 & 0.089 \\
ILIRI & 1.466 & 0.146 \\
LGALS7 & 1.655 & 0.101 \\
\hline
\end{tabular}

Table 3.6: Effect of gene regulation on TRM in HSCT patients. Genes significantly regulated $(\mathrm{p}<0.05)$ in patients that died due to transplant and patients who died due to other causes and patients who are still alive. Additionally, genes that show a trend of significance $(\mathrm{p}<0.15$; n.s.) are listed. $\mathrm{t}$-values represent the size of the relative difference to variation of the sample data; $\mathrm{t}>0$ shows upregulation and $\mathrm{t}<0$ shows downregulation.

The mRNA expression of the chemokine receptor, $C X 3 C R 1$ was significantly higher in patients that died due to TRM, compared to patients that died due to other causes or were still alive. Furthermore, CXCL8, ILIR1, LGALS7 and the DNAM-1 ligand, PVRL2 were also increased in those patients, though they did not reach statistical significance (Table 3.6). Interestingly, the expression of CX3CRlwas increased in severe aGvHD as well, suggesting a correlation between extent of disease and death, however the expression of $C X C L 8$ was contrary to that, whereby an increased expression was associated with death, however the aGvHD severity was associated with a reduced mRNA expression.

\subsubsection{Effect of time period after transplant $(<100$ days and $>100$ days $)$ on gene expression patterns of the selected genes}

Here we studied the gene expression patterns in biopsies that were taken at a later time point after transplant (>100 days) compared to biopsies that were taken $<100$ days after transplant. The time period between transplant and day the biopsy was taken could be considered to be correlated with 
either severity of the disease, or delayed aGvHD onset. It would be interesting to determine the expression patterns of genes that could be associated with this delayed onset.

\begin{tabular}{ccc}
\hline Genes & t-value & p-value $(<\mathbf{0 . 0 5})$ \\
\hline $\boldsymbol{I L 3 3}$ & -2.400 & $\mathbf{0 . 0 1 8}$ \\
\hline & & p-value $(<\mathbf{0 . 1 5})$ \\
\hline CXCL16 & 1.647 & 0.120 \\
CXCL8 & -1.522 & 0.130 \\
LGALS7 & 1.542 & 0.125 \\
CD226 & -1.762 & 0.081 \\
(DNAM-1) & -1.840 & 0.068 \\
\hline
\end{tabular}

Table 3.7: Gene regulation based on time of biopsy taken post transplant. Genes significantly regulated $(\mathrm{p}<0.05)$ in biopsies taken < day 100 post transplant and biopsies taken $>100$ days. Additionally, genes that show a trend of significance $(\mathrm{p}<0.15$; n.s. $)$ are listed. $\mathrm{t}$-values represent the size of the relative difference to variation of the sample data; $\mathrm{t}>0$ shows upregulation and $\mathrm{t}<0$ shows downregulation.

The time of biopsy after transplant did not affect the gene expression patterns strongly. Most genes were not regulated, however we observed a downregulation of the Th2 cytokine IL33 in biopsies that were taken at a later time point. In addition, HCLS1 and the NK receptor CD226 were also reduced but were not significant.

\subsubsection{Gene expression in patients with no steroid" treatment}

In order to eliminate the effects of steroid treatment on the gene regulation in the patient biopsies, we separated the patients that were not treated with steroids or those who were treated with a dosage of $\leq 20 \mathrm{mg} / \mathrm{kg}$ steroids ( $\mathrm{n}=69$ ), from those who underwent steroid treatment. This cut off value for the two groups was pre-defined by clinicians. Within the group of patients with no steroid (or low) treatment, we studied the changes in gene expression patterns based on the different variables.

* Patients that were treated with a $20 \mathrm{mg} / \mathrm{kg}$ or less steroid dosage and patients that were not treated with steroids. 


\subsubsection{Effect of clinical GI GvHD grade on gene expression patterns of the selected genes in patients with no steroid treatment ${ }^{*}$}

In table 3.8, we compared the gene expression during clinical GI aGvHD in patients with no or less steroids. Several genes were significantly increased based on the clinical scores in patients with aGvHD. Genes that were most strongly regulated were the chemokine receptors, CCR5, CCR4, CCRI and CXCR3. In addition, CXCR4 and the chemokines CCL4, CCL5 and CXCL8 were significantly increased. The Th1 and Th2 cytokines IL2 and IL6 respectively were also increased. Other regulated genes included genes associated with activation and regulation of Tregs, IL2RA and TGFB1, genes associated with activation of B and T cells, PSTPIP1, PIK3AP1, PTPN7, HCLS1, FCGR3 and FCERIG and genes regulated by IFN- $\gamma, L S T 1$ and MSRI.

Considering most of these genes are not regulated based on the histological scores, it is perceivable that these genes are important for the clinical symptoms in patients such as skin rash, weight loss etc., most likely due to inflammation, and a heightened immune response post transplant. Activation of alloreactive T cells and infiltration of $\mathrm{T}$ cells in the GI tissue, as a result of inflammation, explains the heightened clinical symptoms. Most of the regulated genes were also regulated in patients with steroids (Table 3.4), suggesting that the administration of steroids had no or little effect on the regulation of these genes. The main difference between the two groups was in the regulation of MSR1, the macrophage scavenger receptor 1. It could be that infiltration of macrophages in the GI tissue, in addition to other immune cells results in the clinical aGvHD symptoms in the patients. 


\begin{tabular}{|c|c|c|}
\hline Genes & t-value & p-value $(<0.05)$ \\
\hline CCR5 & 3.482 & 0.0006 \\
\hline CCR4 & 3.478 & 0.0006 \\
\hline$C X C R 3$ & 3.416 & 0.0008 \\
\hline CCR1 & 3.314 & 0.0012 \\
\hline FCGR3 & 3.257 & 0.0014 \\
\hline HCLS1 & 2.948 & 0.0037 \\
\hline TGFB1 & 2.862 & 0.0048 \\
\hline FCER1G & 2.861 & 0.0048 \\
\hline$I L 2 R A$ & 2.746 & 0.0067 \\
\hline VCAM1 & 2.726 & 0.007 \\
\hline MSR1 & 2.703 & 0.008 \\
\hline IL6 & 2.631 & 0.009 \\
\hline LST1 & 2.583 & 0.011 \\
\hline$I L 2$ & 2.464 & 0.014 \\
\hline CXCR4 & 2.241 & 0.026 \\
\hline CCL4 & 2.221 & 0.028 \\
\hline CXCL8 & 2.123 & 0.035 \\
\hline PIK3API & 2.117 & 0.036 \\
\hline PTPN7 & 2.106 & 0.041 \\
\hline HTRA1 & 2.052 & 0.042 \\
\hline CCL5 & 2.051 & 0.042 \\
\hline \multirow[t]{2}{*}{ PSTPIP1 } & 2.049 & 0.042 \\
\hline & & p-value $(<0.15)$ \\
\hline ClQTNF7 & 1.78 & 0.077 \\
\hline IL15 & 1.67 & 0.097 \\
\hline$U B D$ & 1.5 & 0.13 \\
\hline CARD11 & 1.47 & 0.14 \\
\hline
\end{tabular}

Table 3.8: Genes regulated in clinically scored GI aGvHD (grade 1-4) biopsies versus no aGvHD (grade 0) in patients with no steroids*. Genes significantly regulated $(\mathrm{p}<0.05)$ based on histological aGvHD in human GI biopsies. Additionally, genes that show a trend of significance $(\mathrm{p}<0.15$; n.s. $)$ are listed. $t$-values represent the size of the relative difference to variation of the sample data; $t>0$ shows upregulation and $\mathrm{t}<0$ shows downregulation. "Patient group consisting of patients who were treated with a $20 \mathrm{mg} / \mathrm{kg}$ or less steroid dosage and those who were not treated with steroids. 


\subsubsection{Effect of histological aGvHD score on gene expression patterns of the selected genes in patients with no steroid treatment ${ }^{*}$}

\subsection{Histological aGvHD grades 2-4 versus no or low aGvHD (grades 0-1)}

\begin{tabular}{ccc}
\hline Genes & t-value & p-value $(<\mathbf{0 . 0 5})$ \\
\hline CX3CR1 & 3.173 & $\mathbf{0 . 0 0 1 8}$ \\
CXCL8 & -2.988 & $\mathbf{0 . 0 0 3}$ \\
CCL4 & -2.638 & $\mathbf{0 . 0 0 9}$ \\
CXCL16 & 2.159 & $\mathbf{0 . 0 3 2}$ \\
IL4 & 2.058 & $\mathbf{0 . 0 4 1}$ \\
PSTPIP1 & -1.973 & $\mathbf{0 . 0 5}$ \\
\hline & & p-value $(<\mathbf{0 . 1 5})$ \\
\hline LILRA5 & -1.914 & 0.057 \\
CXCR4 & -1.612 & 0.109 \\
CXCL9 & -1.516 & 0.138 \\
\hline
\end{tabular}

Table 3.9: Genes regulated in severe histological GI aGvHD (grade 2-4) versus no or low aGvHD (grade 0-1) in patients with no steroids*. Genes significantly regulated $(\mathrm{p}<0.05)$ based on histological aGvHD in human GI biopsies. Additionally, genes that show a trend of significance $(\mathrm{p}<0.15$; n.s.) are listed. t-values represent the size of the relative difference to variation of the sample data; $\mathrm{t}>0$ shows upregulation and $\mathrm{t}<0$ shows downregulation. "Patients group consisting of patients who were treated with a $20 \mathrm{mg} / \mathrm{kg}$ or less steroid dosage and those who were not treated with steroids.

Since the administration of steroids in patients, as part of treatment post-transplant has a significant effect on the gene expression, we studied the regulation of our selected genes in patients that were not given steroids. In patient biopsies with histological grade 2-4, we observed a significant upregulation of the Th2 cytokine, IL4 and the chemokines and chemokine receptor, CX3CRI and CXCL16 respectively. The chemokines CCL4 and CXCL8, and PSTPIPI were decreased in patients with severe aGvHD. Moreover, LILRA5, CXCR4 and CXCL9 were decreased in patients with severe aGvHD but did not reach statistical significance (Table 3.9). Interestingly, these genes were regulated in the same way even in the presence of steroids, suggesting that the presence of steroids did not have a significant effect on these genes. On the other hand, the genes ENPPI and the NKG2D ligand, ULBP3 were significantly regulated in the presence of steroids (Table 3.5).

No significant changes in the expression of genes were observed in this subgroup analysis between patients with histological aGvHD (grade 1-4) and patients without aGvHD (grade 0). 


\subsubsection{Effect of gene regulation on transplant related mortality (TRM) in HSCT patients with no steroid treatment ${ }^{*}$}

\begin{tabular}{ccc}
\hline Genes & t-value & p-value $(<\mathbf{0 . 0 5})$ \\
\hline LGALS7 & 2.711 & $\mathbf{0 . 0 0 7}$ \\
PVRL2 & 2.538 & $\mathbf{0 . 0 1 2}$ \\
IL1R1 & 2.474 & $\mathbf{0 . 0 1 4}$ \\
CXCL8 & 2.466 & $\mathbf{0 . 0 1 4 8}$ \\
CIQTNF7 & 2.194 & $\mathbf{0 . 0 2 9}$ \\
HTRA1 & 2.103 & $\mathbf{0 . 0 3 6}$ \\
TGFB1 & 2.05 & $\mathbf{0 . 0 4 1}$ \\
\hline & & p-value $(<\mathbf{0 . 1 5})$ \\
\hline IL2RA & 1.91 & 0.058 \\
CCL4 & 1.819 & 0.07 \\
TGFB2 & 1.702 & 0.09 \\
ILIR2 & 1.592 & 0.113 \\
CCR4 & 1.567 & 0.119 \\
\hline PSTPIP1 & 1.477 & 0.141 \\
\hline CXCL9 & 1.444 & 0.15 \\
\hline
\end{tabular}

Table 3.10: Effect of gene regulation on TRM in patients with no steroids. Genes significantly regulated $(\mathrm{p}<0.05)$ based on histological aGvHD in human GI biopsies. Additionally, genes that show a trend of significance $(\mathrm{p}<0.15$; n.s. $)$ are listed. $\mathrm{t}$-values represent the size of the relative difference to variation of the sample data; $t>0$ shows upregulation and $\mathrm{t}<0$ shows downregulation. Patients group consisting of patients who were treated with a $20 \mathrm{mg} / \mathrm{kg}$ or less steroid dosage and those who were not treated with steroids.

The presence of steroids had a significant effect on regulation of genes associated with TRM. Several genes were regulated differently in patients that died due to transplant when no steroids were administered. The DNAM-1 ligand, PVRL2 was higher in patients that died due to transplant compared to patients that were still alive or those that died due to other causes. Moreover, several genes associated with innate immune responses, such as C1QTNF7, LGALS7 and HTRA1 were also increased. The expression of several genes that were associated with clinical aGvHD symptoms in patients (Table 3.8), were also correlated with death due to transplant, such as HTRA1, CXCL8, TGFB1, IL2RA and PSTPIPI (Table 3.10).

\subsubsection{Regulation of NK receptors and their ligands during clinical GI aGvHD}

To determine how the genes KLRK1, CD226 and their ligands are regulated in aGvHD in humans, we analyzed the gene expression of these genes as univariate analysis. 


\subsubsection{Regulation of $K L R K 1$ and its ligands in clinical GI aGvHD}
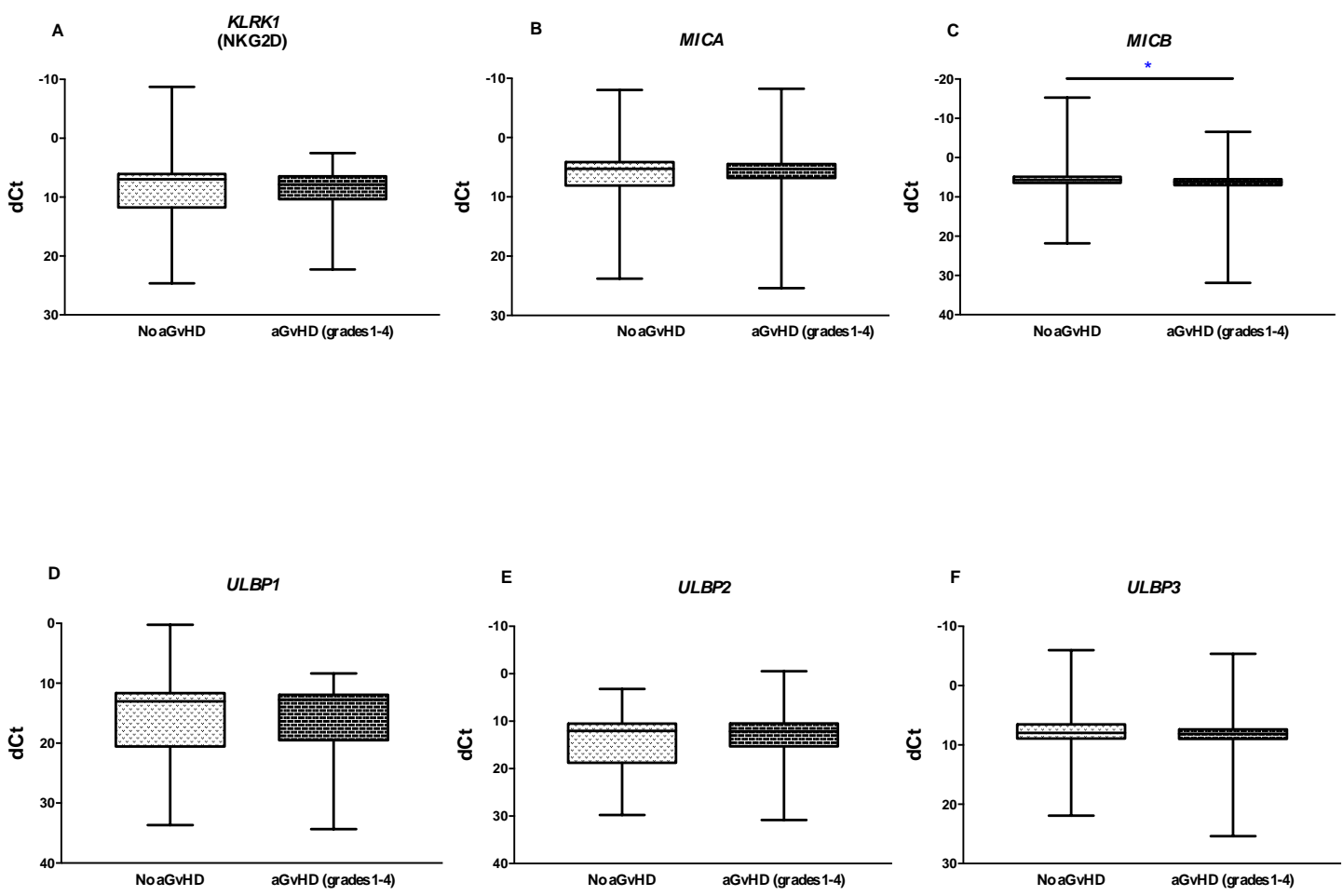

Figure 3.22: Gene expression of KLRK1 (NKG2D) and its ligands in clinical GI aGvHD biopsies.

Regulation of genes in human GI aGvHD (grades 1-4) compared to patients with no GvHD (grade 0). None of the genes reached statistical significance. A. KLRKI $(\mathrm{p}=0.548)$ B. MICA $(\mathrm{p}=0.505)$ C. $M I C B(\mathrm{p}=0.049)$ D. $U L B P 1(\mathrm{p}=0.828)$ E. $U L B P 2(\mathrm{p}=0.972)$ F. $U L B P 3(\mathrm{p}=0.322)$

KLRK1 mRNA expression was upregulated during aGvHD in patients, but was not statistically significant. We observed differential expression patterns of its ligands, of which $M I C B$ was significantly downregulated.

\subsubsection{Regulation of $C D 226$ and its ligands in clinical GI aGvHD}
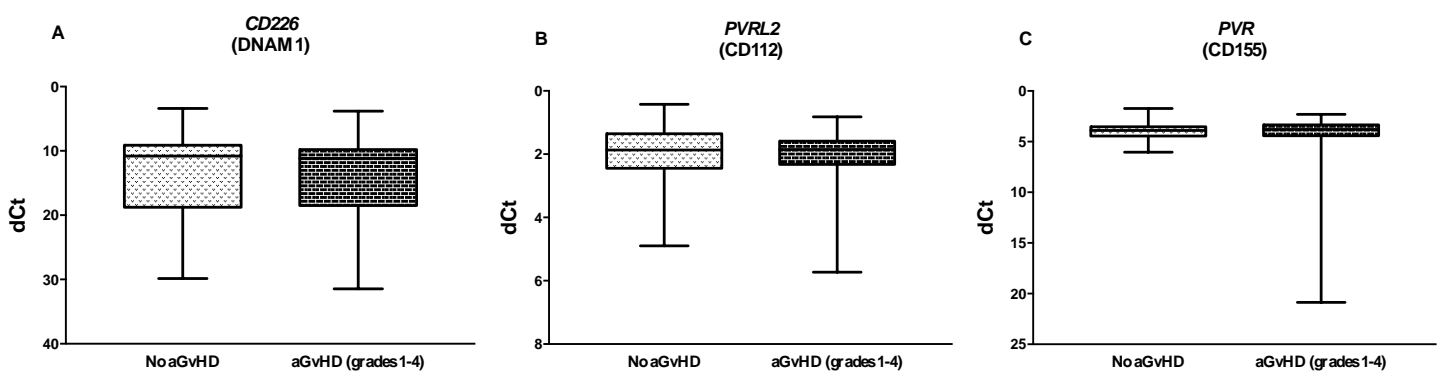

Figure 3.23: Gene expression of $C D 226$ and its ligands in clinical GI aGvHD biopsies. Regulation of genes in human GI aGvHD (grades 1-4) compared to patients with no GvHD (grade 0). None of 
the genes reached statistical significance. Increased mRNA expression of A. CD226 $(\mathrm{p}=0.394)$ B. PVRL2 $(\mathrm{p}=0.471)$ and $\mathbf{C} . P V R(\mathrm{p}=0.59)$ were observed.

The expression of CD226 and its ligands was not statistically different, but a trend of downregulation was observed a trend of upregulation of $C D 226$, which followed a similar trend in expression as in the mouse during aGvHD.

\subsubsection{Effect of regulation of KLRK1, CD226 and their ligands on TRM in HSCT patients with clinical GI aGvHD}

A

MICA

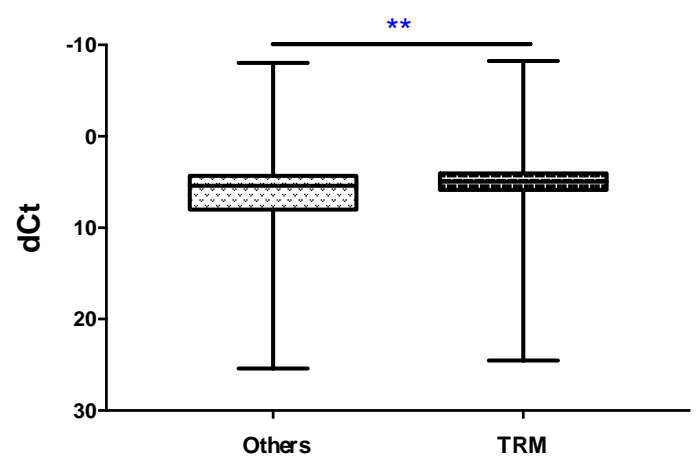

B

PVRL2

(CD112)

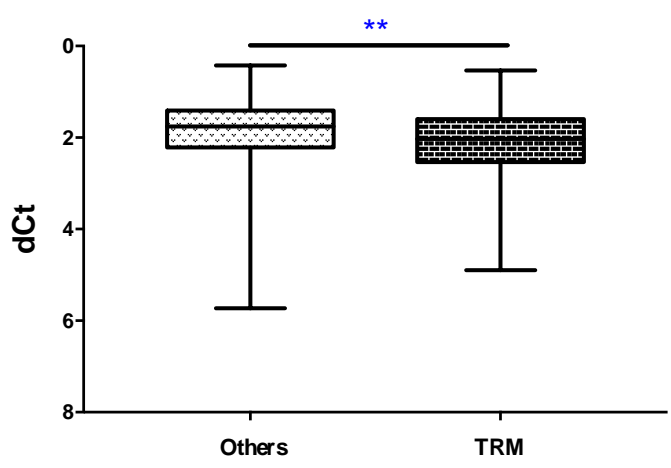

Figure 3.24: Effect of gene regulation of $K L R K 1, C D 226$ and their ligands on TRM in human GI aGvHD.

The p-values and fold changes are shown for all the genes, however only the significant results are plotted in the graphs. Increased mRNA expression of A. MICA and downregulation of B. PVRL2 was observed in patients that died due to TRM compared to patients who are still alive or died due to unrelated causes (others).

On performing a univariate analysis on the expression of NK receptor genes and their ligands in patients, we observed that patients who died due to transplant related causes had a higher expression of MICA and a lower PVRL2 expression compared to patients that were still alive or those who died due to other unrelated causes (Figure 3.24). 


\begin{tabular}{ccc}
\hline Genes & Fold change & p-value $(<\mathbf{0 . 0 5})$ \\
\hline MICA & $\mathbf{5 . 1 5}$ & $\mathbf{0 . 0 2 7}$ \\
PVRL2 & $\mathbf{0 . 8 4}$ & $\mathbf{0 . 0 0 9}$ \\
\hline & & $\begin{array}{c}\text { p-value } \\
(>\mathbf{0 . 0 5} ; \text { n.s. })\end{array}$ \\
\hline KLRK1 & 1.66 & 0.647 \\
MICB & 2.22 & 0.548 \\
CD226 & 0.47 & 0.974 \\
$U L B P 1$ & 0.113 & 0.592 \\
$U L B P 2$ & 0.119 & 0.567 \\
$U L B P 3$ & 0.141 & 0.477 \\
\hline
\end{tabular}

Table 3.11: Effect of gene regulation of KLRK1, CD226 and their ligands on TRM in clinical GI aGvHD. Genes significantly regulated $(\mathrm{p}<0.05)$ based on histological aGvHD in human GI biopsies. Additionally, genes that show a trend of significance $(\mathrm{p}>0.05$; n.s. $)$ are listed. $t$-values represent the size of the relative difference to variation of the sample data; $t>0$ shows upregulation and $\mathrm{t}<0$ shows downregulation.

\subsubsection{Effect of steroids on the regulation of KLRK1, CD226 and their ligands in clinical GI aGvHD}

A

MICA

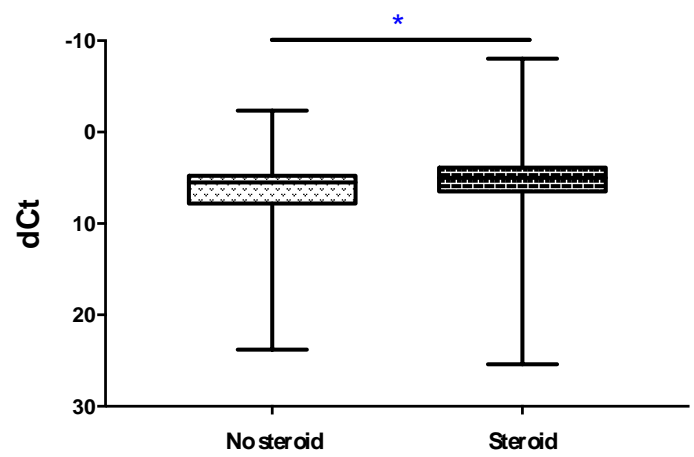

C

CD226

(DNAM-1)

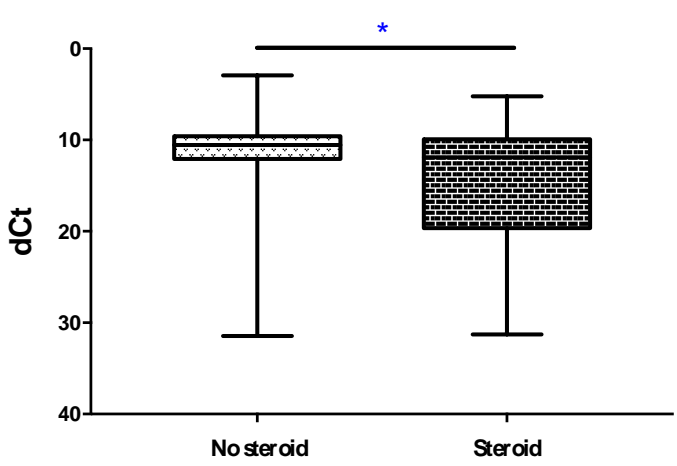

B

ULBP1

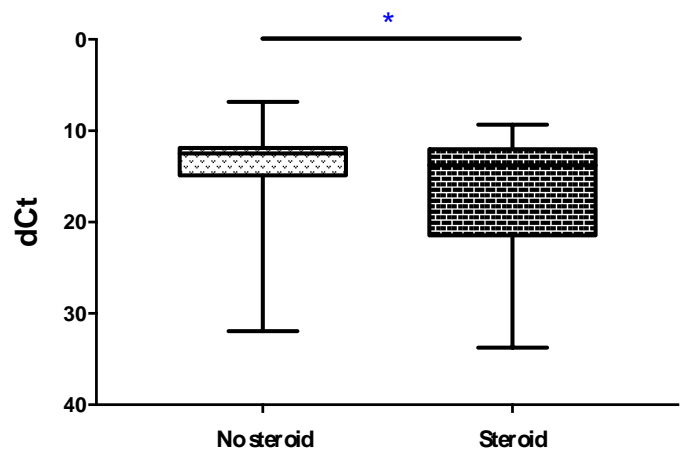

D
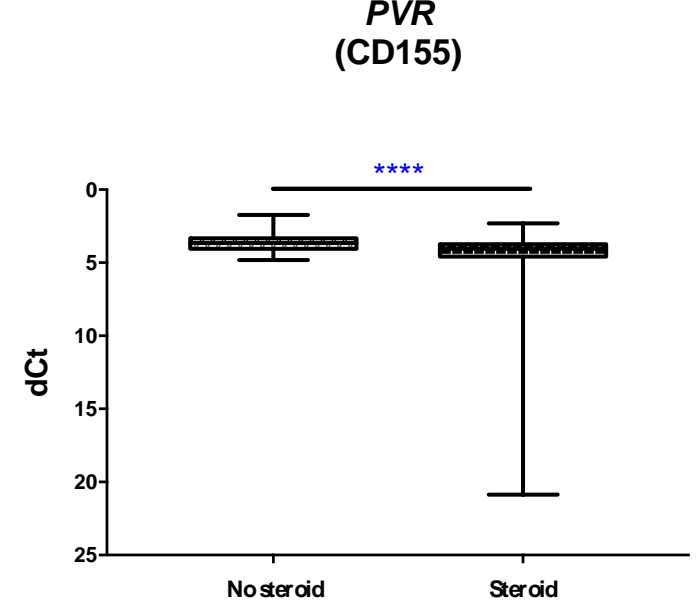
Figure 3.25: Effect of steroid on gene expression of KLRK1, CD226 and their ligands in human GI aGvHD.

The p-values and fold changes are shown for all the genes, however only the significant results are plotted in the graphs. Increased mRNA expression of A. MICA and downregulation of B. ULBP1, C. CD226 and D. $P V R$ was observed in patients that were treated with steroids compared to patients who were not treatment with steroids.

In patients that were treated with steroids, we observed a higher mRNA expression of MICA and a lower expression of $U L B P 1$, compared to patients that were not given steroids as part of treatment after transplant. Similarly, patients who received steroids also showed a significant reduction in the expression of $C D 226$ as well as $P V R$, compared to patients who received no steroids.

\begin{tabular}{ccc}
\hline Genes & Fold change & p-value $(<\mathbf{0 . 0 5})$ \\
\hline MICA & $\mathbf{2 . 8 4}$ & $\mathbf{0 . 0 1 9}$ \\
ULBP1 & $\mathbf{0 . 2 2}$ & $\mathbf{0 . 0 2 2}$ \\
CD226 & $\mathbf{0 . 2 9}$ & $\mathbf{0 . 0 5}$ \\
$\boldsymbol{P} \boldsymbol{V R}$ & $\mathbf{0 . 6 2}$ & $\mathbf{0 . 0 0 0 1}$ \\
\hline & & p-value \\
& & (> 0.05; n.s. $)$ \\
\hline$K L R K 1$ & 2.84 & 0.682 \\
$M I C B$ & 0.22 & 0.126 \\
$U L B P 2$ & 0.62 & 0.229 \\
$U L B P 3$ & 2.84 & 0.603 \\
$P V R L 2$ & 0.22 & 0.262 \\
\hline
\end{tabular}

Table 3.12: Effect of steroids on the regulation of $K L R K 1, C D 226$ and their ligands in clinical GI aGvHD. Genes significantly regulated $(\mathrm{p}<0.05)$ in patients who were treated with steroids compared to those who were not treated with steroids. Additionally, genes that show a trend of significance $(\mathrm{p}<$ 0.05 ; n.s.) are listed. $t$-values represent the size of the relative difference to variation of the sample data; $\mathrm{t}>0$ shows upregulation and $\mathrm{t}<0$ shows downregulation. 


\section{Discussion}

\subsection{Comparison of gene expression in different target organs of the same species}

\subsubsection{Comparison of genes associated with different immune responses in the different aGvHD mouse target tissues compared to healthy controls}

We compared the differences in regulation patterns of cytokine and chemokine genes associated with the different immune responses in the mouse tissues affected by aGvHD, compared to healthy control tissues. In Figure 4.1, the hierarchical cluster shows two distinct groups; the first group depicts a close relationship between gene expression patterns in the small and large intestine $\left(\mathrm{p}<0.0001, \mathrm{R}^{2}=0.5619\right)$ followed by the spleen $\left(\mathrm{p}=0.003, \mathrm{R}^{2}=0.3275\right)$ and lung $(\mathrm{p}=0.048$, $\mathrm{R}^{2}=0.056$ ), whereas the second group shows the expression patterns between the skin and liver ( $\left.p=0.0411, \mathrm{R}^{2}=0.059\right)$ followed a similar pattern of expression. A number of genes were similarly regulated in the different tissues. Several genes were upregulated in all the tissues in mice with aGvHD compared to healthy control mice, such as Ifng, Il2ra, Il6 and Ccrl, whereas a number of genes were downregulated in the different tissues, such as Ccl5, Cx3cll, Tgfb2 and Cxcr4. Other genes were found to be regulated in the same direction in all but one tissue, or in some instances showed a mixed pattern of expression. For example, $C x c r 3$ was significantly upregulated in mice with aGvHD in all the tissues, but was downregulated in the liver, compared to healthy controls.

Overall, we observed that in mouse aGvHD, the Th1, Th2 and Th17 cytokines were mostly regulated predominantly in the lung and large intestine of aGvHD mice compared to controls. Moreover, the genes associated with Tregs were also significantly regulated mainly in the liver, lung and both small and large intestines. The trend in expression was similar in the different tissues, whereby a significantly increased Th1, Th2 and Th17 cytokine expression, in addition to an increase in genes associated with Tregs was observed in mice with aGvHD compared to healthy controls. 

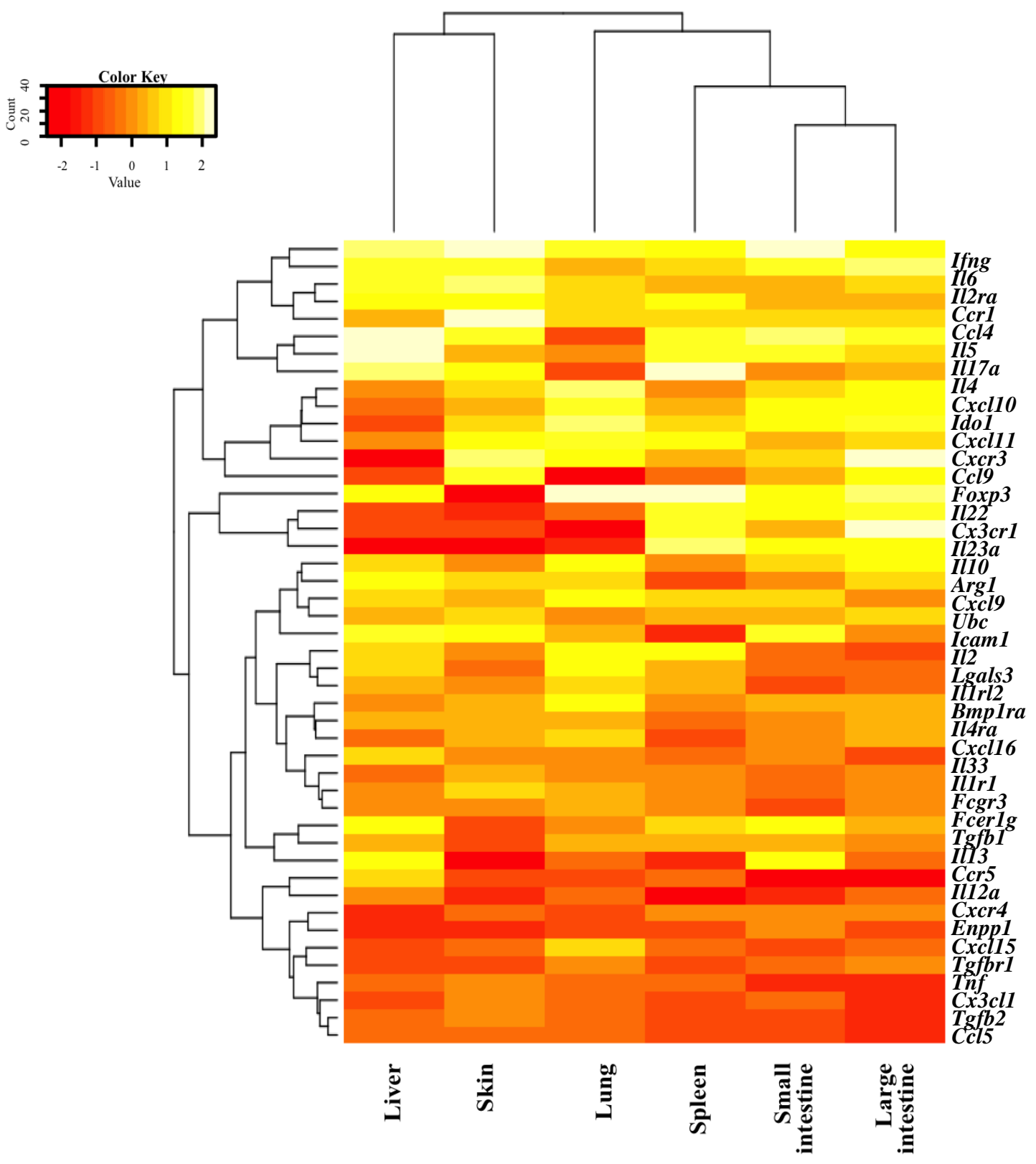

Figure 4.1: Hierarchical cluster of genes associated with immune responses in the different tissues of mouse aGvHD compared to healthy controls.

The $\mathrm{x}$-axis represents cluster of gene expression patterns in different tissues. The $\mathrm{y}$-axis represents clusters of regulated genes during aGvHD. The tissues are clustered in two main groups; large and small intestine, lung and spleen versus liver and skin. The color key represents the scaled fold changes from -2 (downregulated) to +2 (upregulated). All fold change values are shown (even those with $\mathrm{p}$-value $=\mathrm{ns}$ )

Interestingly, the expression patterns of the chemokines and their receptors were more consistent in the different target organs. The chemokines $\mathrm{Cxcl9}$, $\mathrm{Cxcl10}$ and $\mathrm{Cxcl11}$ were mostly regulated in all the tissues. Previous studies have elucidated that the mRNA expression of the chemokine receptor, 
Cxcr3 (Bouazzaoui et al., 2009) and its ligands, $\mathrm{Cxcl9}$, Cxcl10 and Cxcll1 are increased in mouse liver during aGvHD (Sadeghi et al. 2013; Ichiba et al. 2003). Our results were in line with the previous data. CXCR-3 is expressed on T cells and is important in recruitment of lymphocytes, and its ligands, CXCL-9, -10 and -11 are induced by IFN- $\gamma$ and TNF- $\alpha$ (Groom and Luster, 2011) and are involved in recruiting leukocytes expressing CXCR-3 to sites of inflammation (Baggiolini, 1998; Flier et al., 2001). CXCL-9 is expressed by effector CD4 ${ }^{+}$Th1 cells and CD8 ${ }^{+}$CTLs, and affects the migration of effector T cells to inflamed tissue during progression of GvHD (Groom and Luster, 2011).

Moreover, the conditioning regimens significantly altered the expression of several chemokines, however the cytokines remained unchanged. Several chemokine receptors such as Ccrl, Ccr4, Ccr5, Cxcr4, Cx3crl and chemokines Ccl4, Ccl5 and Ccl9 were regulated due to the preconditioning, and their regulation was further augmented due to aGvHD. CCL-4, CCL-5 (RANTES) and CCL-9 are expressed by several different cell types such as T cells, NK cells, endothelial cells, epithelial cells, fibroblasts, neutrophils and APCs, and are associated with Th1 type responses. They bind to their receptors CCR-1, CCR-4 and CCR-5 that are expressed on NK cells, T cells, macrophages and DCs (Wysocki et al., 2005a). These chemokines are induced by inflammatory cytokines such as IFN- $\gamma$ and TNF- $\alpha$ (Moser et al., 2004). CCR-5 serves as a coreceptor for HIV infection (Liu et al., 1996) and is associated with Th1 responses (Sallusto et al., 1998), whereas CCR-4 is expressed by Th2 cells (Campbell et al., 1999; Sallusto et al., 1998). Interestingly, Ccrl, Ccr5, Ccl4 and Ccl9 were significantly increased in the different mouse aGvHD tissues compared to healthy controls, whereas, the expression of $\mathrm{Ccr} 4$ and $\mathrm{Ccl} 5$ was significantly downregulated.

Similarly, the expression of Cxcl16 was significantly increased in the aGvHD mouse compared to the controls, however Cxcl15 and Cxcr4 were significantly reduced. CXCL-16 is expressed both on lymphoid and non-lymphoid tissues (Wysocki et al., 2005a). CXCR-4 is a chemokine receptor that binds to its ligand CXCL-12 or stromal derived factor-1 (SDF-1) that is involved in trafficking of hematopoietic stem cells (Moll and Ransohoff, 2010). Increased CXCR-4 expression could result in better engraftment (Brenner et al., 2004), suggesting that an increased Cxcr4 expression is beneficial for an attenuated aGvHD response. High levels of CX3CL1 have been associated with intestinal damage due to recruitment of $\mathrm{CD}^{+} \mathrm{T}$ cells to the intestine during aGvHD (Ueha et al., 2007). A reduction of $\mathrm{CD}^{+} \mathrm{T}$ cells was observed on administration of a CX3CL1 antibody, which was associated with improved survival rates and reduced aGvHD (Ueha et al., 2007). However, we observed a downregulation of $\mathrm{Cx} 3 \mathrm{cll}$ in mice with aGvHD compared to the BM controls, whereas its receptor, $\mathrm{Cx3}$ crl was significantly increased in aGvHD tissues compared to the BM control mouse. Moreover, we observed an increased expression of Icaml in the aGvHD tissues compared 
to the healthy controls. ICAM1 is an adhesion molecule expressed on endothelial cells and are critical for the migration of leukocytes to tissues during inflammation (Ren et al., 2010). IL-15 is a critical mediator for T cell function during aGvHD (Blaser et al., 2005). In aGvHD affected tissues, we observed $I l 15$ to be downregulated compared to healthy controls.

Several gene expression profiling studies have been carried out to determine the expression patterns of cytokines and chemokines previously (Jaksch et al., 2005; Poloni et al., 2011; Tanaka et al., 1995). Our study highlights the differences in the gene expression patterns between the different target aGvHD tissues in mouse with aGvHD. An important aspect of our findings is that mice with aGvHD had an exacerbated chemokine regulation, in addition to the proinflammatory cytokines. Thus aGvHD in the mouse tissues was associated with a stronger regulation in the chemokine genes across the different tissues, compared to several cytokines.

Furthermore, we observed a distinct expression pattern of the genes associated with Tregs. In the large intestine, we observed an upregulation of most Treg associated genes such as Foxp3, Il2ra, Il10, Ido1, Arg1, Tgfb1, Illrl2 and Lgals3 in aGvHD mouse compared to healthy controls or BM controls. Moreover, several genes were also significantly upregulated in the liver, lung and small intestine, including Il2ra, Argl, Idol, Illrl2 and Tgfbl. Aside from the presence of Foxp3 mRNA, which is the marker for Tregs, the regulation of the other genes associated with Tregs were strongly indicative of an expanding Treg population in the different target tissues, mainly the large intestine. These genes included $I l 2 \mathrm{ra}$, encoding $\mathrm{Cd} 25$, which is expressed by Tregs and activated $\mathrm{T}$ cells and Il1rl2 and Lglas3 which are expressed on human Treg subsets (Ocklenburg et al. 2006, Pfoertner et al. 2006). LGALS3 can change the cytokine profile of T cells and is therefore involved in regulated effector cells and homeostasis of immune cells (Demetriou et al., 2001). Furthermore, Idol is linked to the differentiation of Tregs and contribute to their suppressive capacity. IDO- 1 is constitutively expressed in the GvHD target organs, lung and intestine, and can be up regulated due to inflammation (Jasperson et al., 2009).

Furthermore, ARG-1, another metabolic enzyme is activated during inflammation and reduces T cell responses at the sites of inflammation (Highfill et al., 2010; Rodriguez et al., 2004) and is important in Treg function. Arginase activity is increased by IL-10. Furthermore bone marrow myeloid-derived stem cells (MDSCs) inhibit GvHD in mouse via an arginase-1 dependent mechanism that is upregulated by IL-13 (Highfill et al., 2010). Finally, we observed an increased mRNA expression of the chemokine receptor $C c r 5$, in the different tissues. $C c r 5$ is a chemotactic receptor for Tregs, and is involved in regulating the balance between Tregs and Th17 cells in sites of inflammation (Zhang et al., 2009). Taken together, these results strongly suggest the expansion 
of a Treg population at sites of tissue inflammation. Based on our results, we postulate that the increasing Treg population is indeed iTregs.

The expression of Treg markers and other genes associated with the activation and regulation of Tregs in these tissues, suggest a possible infiltration of $\mathrm{T}$ cells, and specifically Tregs in the tissue sites of Foxp3 mRNA expression. This could be due to an increased need for the suppressive function of Tregs in response an increased inflammation as a result of tissue injury in the large intestine. Our results are similar to previous results that found Foxp $3^{+}$cells to be increased in intestinal mucosa during aGvHD (Lord et al., 2011), as well as in patients with a more severe grade of GvHD (Ratajczak et al., 2010). On the other hand, previously several reports showed an inverse relationship between expression of Foxp3 mRNA and progression of GvHD. For example, a decreased Treg frequency was observed in patients that had severe aGvHD or incidence of cGvHD (Magenau et al., 2010; Li et al. 2010; Zorn et al. 2005). Similarly, increase in the number of donor Tregs were associated with a lower incidence of cGvHD (Miura et al., 2004) and aGvHD severity (Miura et al. 2004; Wolf et al. 2007; Rezvani et al. 2006). Furthermore, the mRNA expression of FOXP3 was increased in patients there were responsive to anti-GvHD therapies (Cuzzola et al. 2012), and inducing selective expansion of Tregs by the daily administration of low doses of IL-2, showed an improvement in clinical cGvHD symptoms in patients (Koreth et al., 2011). An explanation for the contradictory results could be due to the inability to discriminate between natural and induced Tregs. It is likely that natural Tregs are decreased during aGvHD, which could explain the therapeutic effects of administered Tregs to reduce GvHD. On the other hand, the increase in Foxp3 mRNA seen in our study in aGvHD mouse compared to healthy and BM controls, as well as previous reports during aGvHD (Lord et al. 2011; Ratajczak et al. 2010), could be due to an increase in induced Treg population. We hypothesize that the depletion of natural Tregs could cause the tissues to induce Tregs that rapidly expand to compensate for the exacerbated inflammation. Perhaps, it is this induced Treg population that we most likely observe in the large intestine. It would be interesting to distinguish the Treg populations in the large intestine, which would give us a more comprehensive explanation about the pathology in the intestine during aGvHD.

Further evidence of a potential iTreg expression in the gut, is the increase in $T g f b 1$ and $T g f b 2$ mRNA expression. Since iTreg are induced in the presence of TGF- $\beta$ (Cobbold et al., 2004), and the TGF- $\beta$ induced Tregs are more stable and functional than nTregs in mice with established autoimmunity (Kong et al., 2012), it may as well be that during aGvHD, an expansion of iTregs is observed in relation with an increased aGvHD response, perhaps to compensate for a decrease in nTreg population. Moreover, it is likely that this mechanism is expansion is mainly tissue mediated. 
The differences in the regulation of genes in different target organs can primarily be attributed to the difference in their expression under normal conditions. Transcriptional patterns and biological processes shared by certain organs could also explain the similarities and differences in gene regulation. Also, genes that are highly correlated or co-expressed possibly belong to the same functional pathway (Stuart et al., 2003). The pathogenesis of aGvHD mainly involves the skin, lung, liver and gastrointestinal tract. We already observed differences in genes expressed in the target organs in the mouse compared to the spleen, which is not a major target of aGvHD. An interesting example was the difference in the regulation of Th1 associated cytokines, which are considered the key regulators of aGvHD. Th1 cytokines, Il2, Tnf and $I l 12 a$ were increased in the aGvHD target organs, whereas they were significantly decreased in the spleen.

In summary, we observed an upregulation of Th1, Th2, Th17 and Treg responses in the different tissues. Several chemokines were also significantly regulated. Many genes showed similarities in their regulation patterns in the different tissues, including Ifng, Il6, Il2ra, Ccrl, Ccl4, Il4 and IcamI which were upregulated in all the different tissues and Ccl5, Cx3cl1, Tgfb2, Tnf, Enpp1 and Cxcr4 that were decreased in all the tissues.

\subsubsection{Comparison of previously identified candidate genes in the different aGvHD target tissues in mouse and rats}

In Figure 4.2, the expression patterns of the previously identified candidate genes were similar in the small intestine and spleen, followed by the large intestine, liver and then lung and skin. However, it is interesting to note that several genes were regulated in the same direction in the different tissues. Several genes such as Lst1, Msr1, Lilra5, Tgm2 and most importantly Ubd and Hcls 1 were upregulated in all the aGvHD tissues compared to healthy controls. LST1 is encoded within the class III region of the MHC, (de Baey et al., 1997) and is implicated in inflammatory and infectious diseases (Mulcahy et al., 2006). It has previously been reported that MSR1 is involved in the regulation of anti-inflammatory responses (Fulton et al., 2006). In addition, LILRA5 is an activating receptor expressed on immune cells and is associated with the release of pro-inflammatory cytokines. (Brown et al., 2004). Moreover, UBD is a downstream regulator of Tregs (Ocklenburg et al., 2006). These genes are all associated with inflammation and thereby increased during aGvHD. 
A

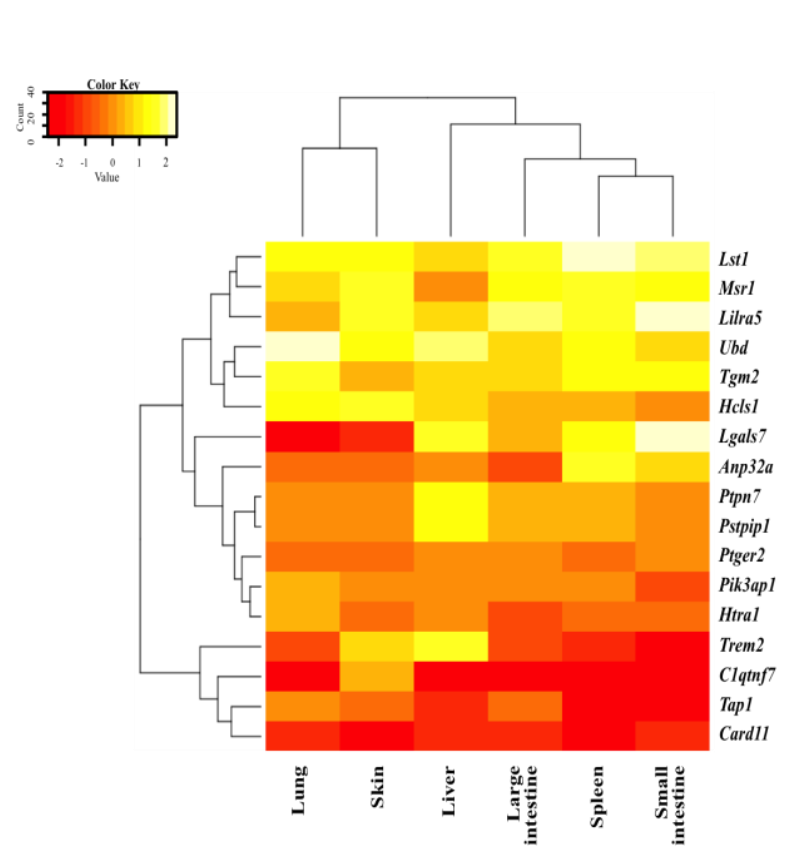

B

Rat aGvHD

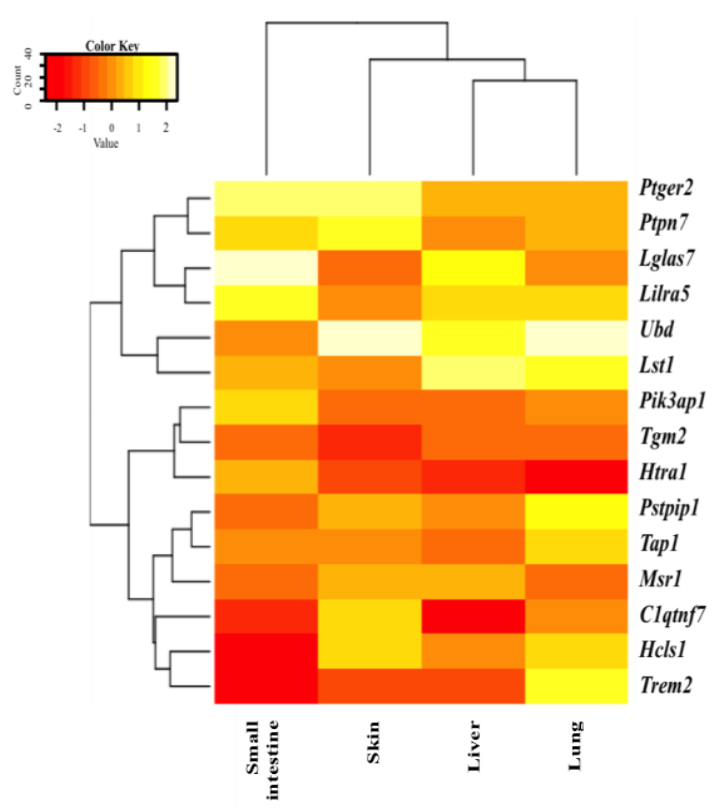

Figure 4.2: Hierarchical cluster of candidate genes in different tissues of mouse and rat aGvHD.

A. Gene expression patterns of candidate genes in different mouse aGvHD tissues compared to healthy control mouse B. Gene expression patterns of candidate genes in different rat aGvHD tissues compared to syngeneic mouse. The $\mathrm{x}$-axis represents cluster of gene expression patterns in different tissues. The y-axis represents clusters of regulated genes during aGvHD. The color key represents the scaled fold changes from -2 (downregulated) to +2 (upregulated). All fold change values are shown (even those with $\mathrm{p}$-value $=\mathrm{ns}$ )

Several of these genes are known to be regulated by IFN- $\gamma$, therefore are induced as a consequence of inflammation. More importantly, Hclsl and other genes, such as Pstpipl and Ptpn7 are also increased in several tissues due to aGvHD compared to controls, though in some cases, the regulation did not reach statistical significance. These genes are associated with activation and regulation of T and B cells. The lack of HCLS1 on B and T cells causes a defect in proliferation and antigen receptor induced apoptosis (Fukuda et al., 1995), whereas PSTPIP1 is involved in the downregulation of CD2 triggered adhesion and activation of T cells (Li et al., 1998), and PTPN7 is involved in T and B lymphocyte development and reduces TCR-induced transcriptional activation (Saxena et al., 1998). These genes are most likely induced as a result of infiltrating $T$ cells in the target tissues or due to the activation of alloreactive $\mathrm{T}$ cells in the organs. Other genes such as Ptger2, were either increased or remained unchanged in the different tissues. Prostaglandin E receptor 2 (PTGER2) or Prostaglandin E2 can modulate cytokine responses by $\mathrm{CD}^{+} \mathrm{T}$ cells directly and increases the expression of IL-17 and decreases IFN- $\gamma$ production by acting on T cells directly (Napolitani et al., 2009), and can inhibit $\mathrm{T}$ cell responses by blocking the proliferation of $\mathrm{T}$ cells (Harris et al., 2002). In addition, PTGER2 is associated with an enhanced secretion of IL-23 
by DCs (Weaver et al., 2007). Also, Pik3apl mRNA expression was consistent between the different target organs in both mouse and rats. Phosphoinositde-3-kinase adaptor protein 1 (PIK3AP1) is involved in the development of B cells (Yamazaki and Kurosaki, 2003) and PI3K activation of NK cells (Ni et al., 2012).

On the other hand, several genes such as Clqtnf7, Tap1, Card11 and Htral were significantly downregulated in the different target tissues. CARD11 is known to mediate activation of $\mathrm{T}$ cells (Pomerantz et al., 2002) and C1q and Tumor Necrosis Factor Related Protein 7 (C1QTNF7) is a protein coding gene that is indirectly impacted by the glucocorticoid receptor gene, $N R 3 C 1$ (Lu et al., 2007). NR3C1 is involved in cellular proliferation and inflammatory responses (Ray and Prefontaine, 1994). In addition, HTRA1 induction in the presence of LPS, increases the incidence of collagen-induced arthritis in mouse (Hou et al., 2013). Moreover TLR-4 ligands induce the expression of HTRA1 in macrophages and fibroblasts (Hou et al., 2013).

Most importantly, these set of previously identified genes are very similarly regulated in the different tissues in mouse aGvHD compared to controls. In contrast, they show a more mixed expression pattern in the different rat aGvHD target organs compared to syngeneic rats. Several genes are differentially regulated between the different target organs such as Trem2, which is upregulated in the small intestine, but downregulated in the liver. TREM2 is downregulated by IFN- $\gamma$ (Zhao and Ivashkiv, 2011) and is known to attenuate macrophage activation (Turnbull et al., 2006). However, several genes were regulated in the same direction, but the regulation of most of these genes in rats was not statistically significant.

In contrast, genes including $\operatorname{Tgm} 2$ were significantly increased in all mouse tissues, but were either reduced or unchanged in the rat. Transglutaminase 2 (TGM2) is induced by retinoic acid and is involved in apoptosis. Furthermore, TGM2 is also involved in inflammation and tumor biology (Griffin et al., 2002). Similarly, Anp32a showed a mixed expression pattern in the different mouse tissues. Acidic Nuclear Phosphoprotein 32 Family, Member A (ANP32A) is protein coding gene involved in several cellular processes including proliferation, differentiation and apoptosis. It is also implicated in tumor suppression, by stimulating apoptosis (Pan et al., 2009). 


\begin{tabular}{|c|c|c|c|c|}
\hline Genes & HUMAN & RAT & $\begin{array}{c}\text { Mouse } \\
\text { aGvHD vs. } \\
\text { healthy } \\
\text { control) }\end{array}$ & $\begin{array}{c}\text { Rat } \\
\text { aGvHD vs. } \\
\text { syngeneic }\end{array}$ \\
\hline ANP32A & down & SKIN & SKIN & SKIN \\
\hline CIQTNF7 & down* & down* & down & NA \\
\hline CARD11 & up* & up* & down* & up \\
\hline CXCL9 & up* & NA & up* & NA \\
\hline HCLS1 & up* & up* & up* & up \\
\hline HTRA1 & down* & down* & down & down \\
\hline LGALS7 & down* & down* & down & down \\
\hline LILRA5 & up* & NA & up* & down \\
\hline LST1 & down* & up* & up* & down \\
\hline MSR1 & up* & up* & up* & down \\
\hline PIK3AP1 & up* & up* & up & down \\
\hline PSTPIP1 & up* & up* & up* & down \\
\hline PTGER2 & up* & up* & down & up \\
\hline PTPN7 & up* & up* & up & up \\
\hline TAP1 & up* & up* & down & down \\
\hline TGM2 & up* & up* & up* & down \\
\hline TREM2 & up* & up* & up & down \\
\hline UBD & up* & up* & up* & up \\
\hline
\end{tabular}

Table 4.1: Comparison of candidate genes regulated in previous human (Norden et al. unpublished data) and rat skin biopsies (Dressel et al., 2013; Novota et al., 2011) and in mouse and rat aGvHD skin in our study.

The regulation patterns of genes from the previous studies (in blue) have been compared to the regulation of the candidate genes in the mouse and rat aGvHD skin in this study. Up shows upregulation of genes in aGvHD tissues compared controls, down shows downregulation. * indicates significant p-values for regulated genes. Genes regulated similarly in the mouse and/or rat aGvHD skin compared to the previous results have been shown in bold.

On comparing the gene expression profiles of the candidate genes from previous studies to the rat and mouse aGvHD skin in our study, we observed that the regulation of several genes was similar in all three studies. We observed a trend of upregulation in the expression of CXCL9, HCLS1, PTPN7 and UBD in all the tissues (Table 4.1). Moreover, HTRA1 and LGALS7 were downregulated in all the tissues affected with aGvHD. The gene expression in mouse aGvHD tissues was compared to healthy controls, and in rat aGvHD tissues, to syngeneic rats.

On comparing the previous results with the genes regulated in mouse aGvHD tissues, we observed a similar trend in upregulation of the genes CXCL9, HCLS1, LILRA5, MSR1, PIK3AP1, PSTPIP1, PTPN7, TGM2 TREM2 and UBD. In addition, a few genes were downregulated in the mouse 
aGvHD tissues compared to the previous results, such as ANP32A, CIQTNF7, HTRA1 and LGLAS7.

Taken together, we have successfully validated the regulation of these novel candidate genes in different aGvHD models and species, making their expression during aGvHD significantly important. These genes could be potential targets for aGvHD therapy. Even though their exact functions during aGvHD are not clear, several of these genes have been implicated in numerous other diseases, and different molecular pathways. Further studies need to be carried out to determine their functional role in HSCT outcome and onset of aGvHD.

In summary, the gene expression patterns in the different target organs were mostly similar, but we observed a few important differences, which could be significant in differentiating therapeutic strategies targeting the different target organs.

\subsection{Differences in gene expression due to conditioning}

Most often mice and rats are subjected to TBI prior to transplant, whereas in the clinic, patients are mostly given chemotherapy, and only a few patients are given TBI (Hülsdünker and Zeiser, 2015). The difference in conditioning regimens between the different species could play an important role in the differences in gene expression. It has been shown previously, that TBI has a role in distinct differences in gene expression of different immune cell populations (Garg et al., 2010). In their study, they reported a significant regulation in the mRNA expression of several inflammatory mediators and chemokines, such as Tnf, Cxcl9, Il2, Il6, Ccl9 and chemokine receptor Cxcr3 in mouse jejunum as a result of TBI (Garg et al., 2010). Furthermore, it was reported that both conditioning and genetic factors altered the expression of chemokines (Mapara et al., 2006).

We observed a number of genes in this study, whose regulation was altered as a result of preconditioning in the mouse tissues. Mostly, the genes were significantly regulated as a result of preconditioning in the $\mathrm{BM}$ control mouse, compared to the healthy control mouse, and this regulation was further augmented in the aGvHD mouse compared to healthy control and $\mathrm{BM}$ control mouse. In some cases, however, the direction of regulation was altered between the two groups. For example, in several genes in the liver, the gene expression of several genes including Ifng, Cxcl9, CxcllO and Il2ra was increased significantly as a result of preconditioning, however this regulation was decreased slightly between BM control mouse and aGvHD mouse (Figure 4.3). In most cases, however the overall expression pattern between in the aGvHD mouse stayed the same between the preconditioning and aGvHD mouse compared to the healthy controls. 
The differences in the gene expression patterns between BM control mice due to preconditioning, and aGvHD mice compared to the BM control mice could be attributed to a number of factors. It is possible the regulation of several genes is exacerbated as a result of preconditioning alone during the initial phase of aGvHD, and is reduced during aGvHD onset. Furthermore, even in the human GI aGvHD biopsies, the type of conditioning, and the presence of steroids were important covariates for gene regulation during aGvHD. For example, the gene expression of CD226, ILIRI, TAP1, TGM 2 and IL6 were affected by type of conditioning regimen, whereas LGALS7 and CX3CR1 mRNA expression was affected by administration of steroids (Table 3.2).

Taken together, we identified genes that are regulated during aGvHD alone, as well as genes that are induced due to preconditioning. This difference in timing of gene resgulation after transplant could be beneficial in developing therapeutic strategies. 
A

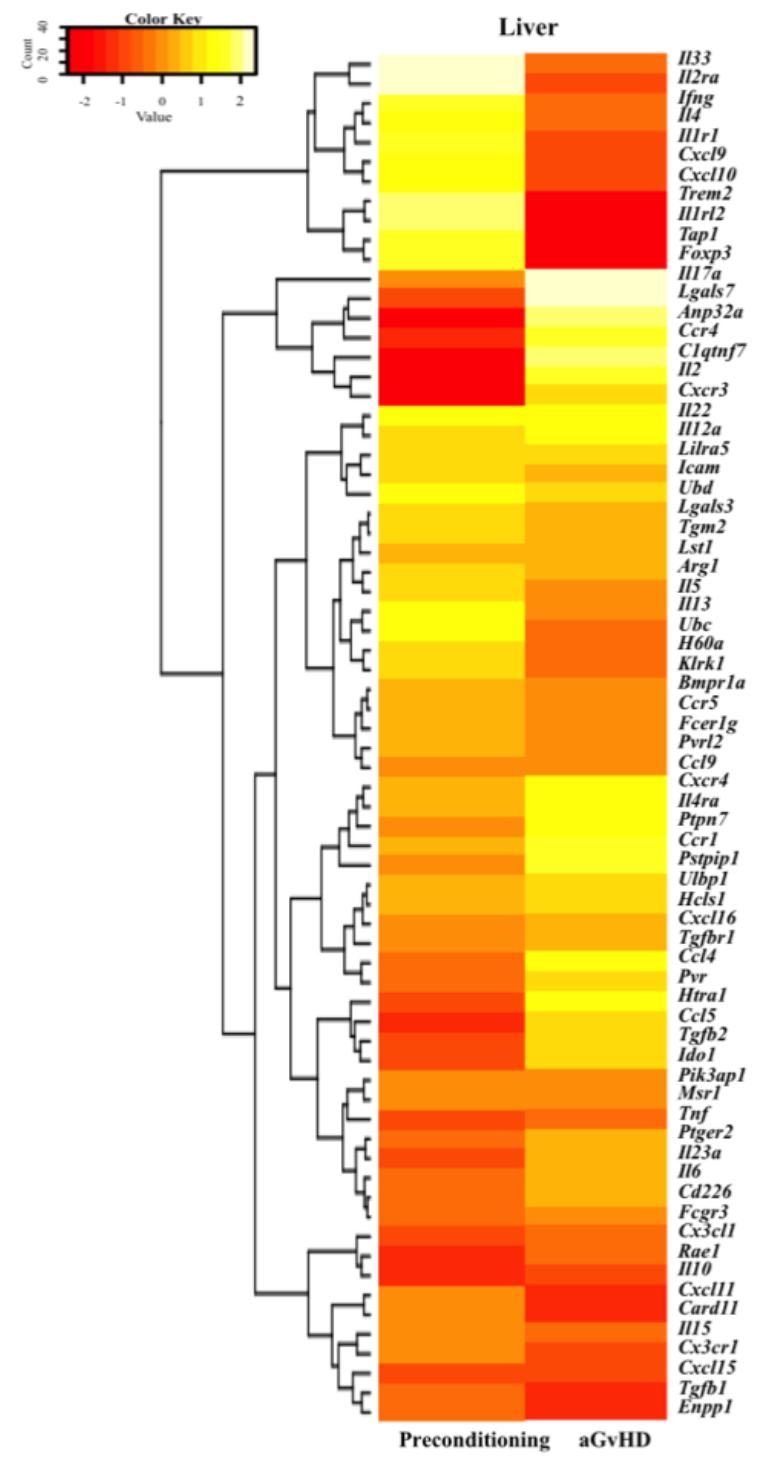

B

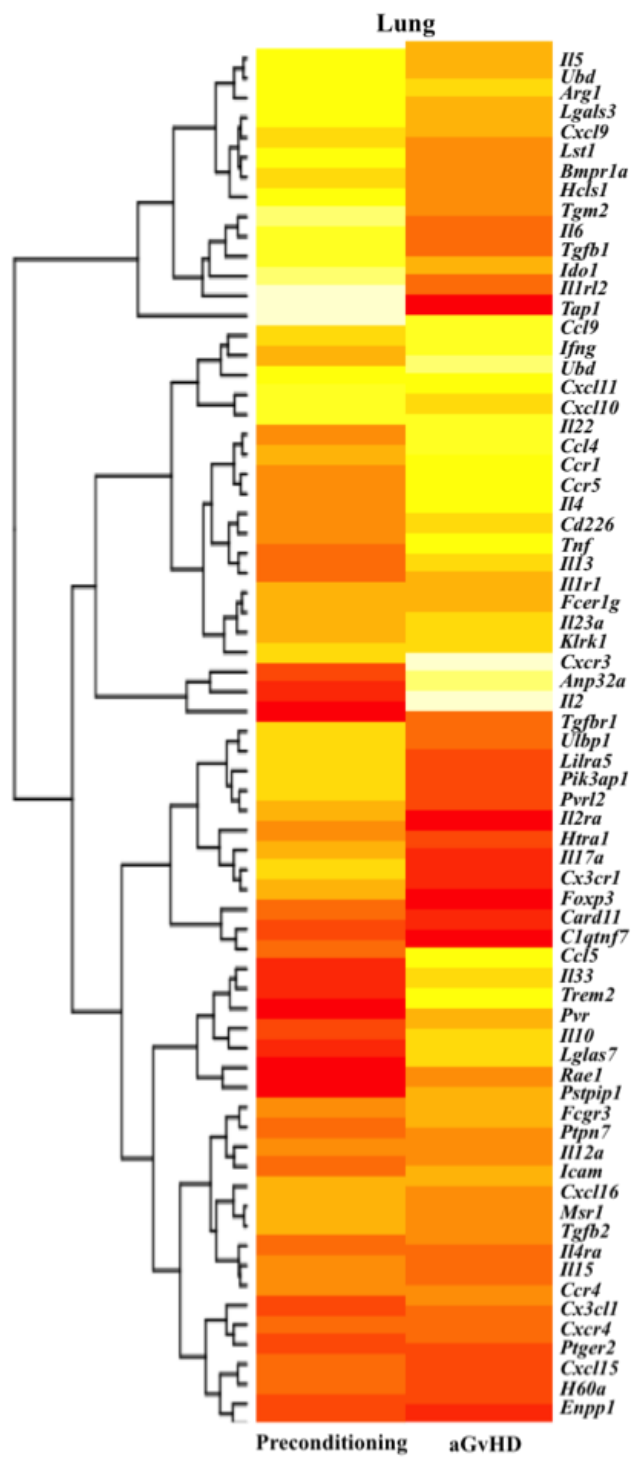


C

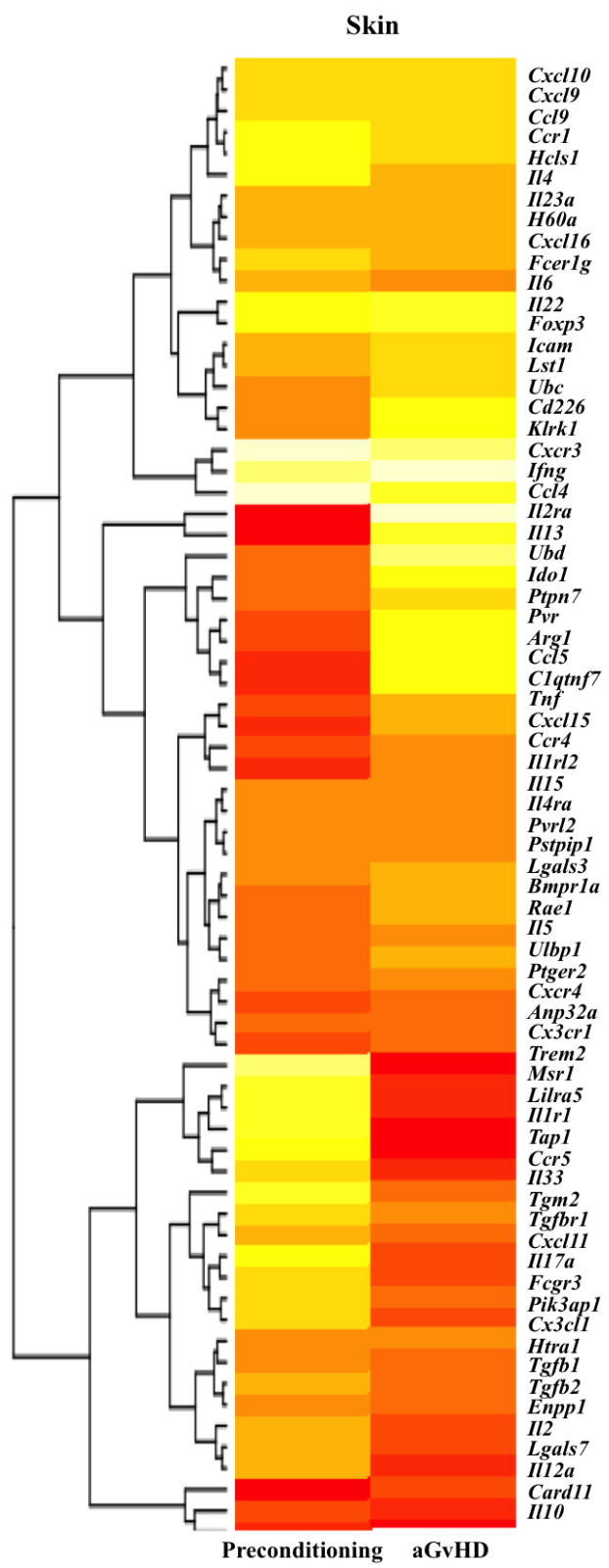

D

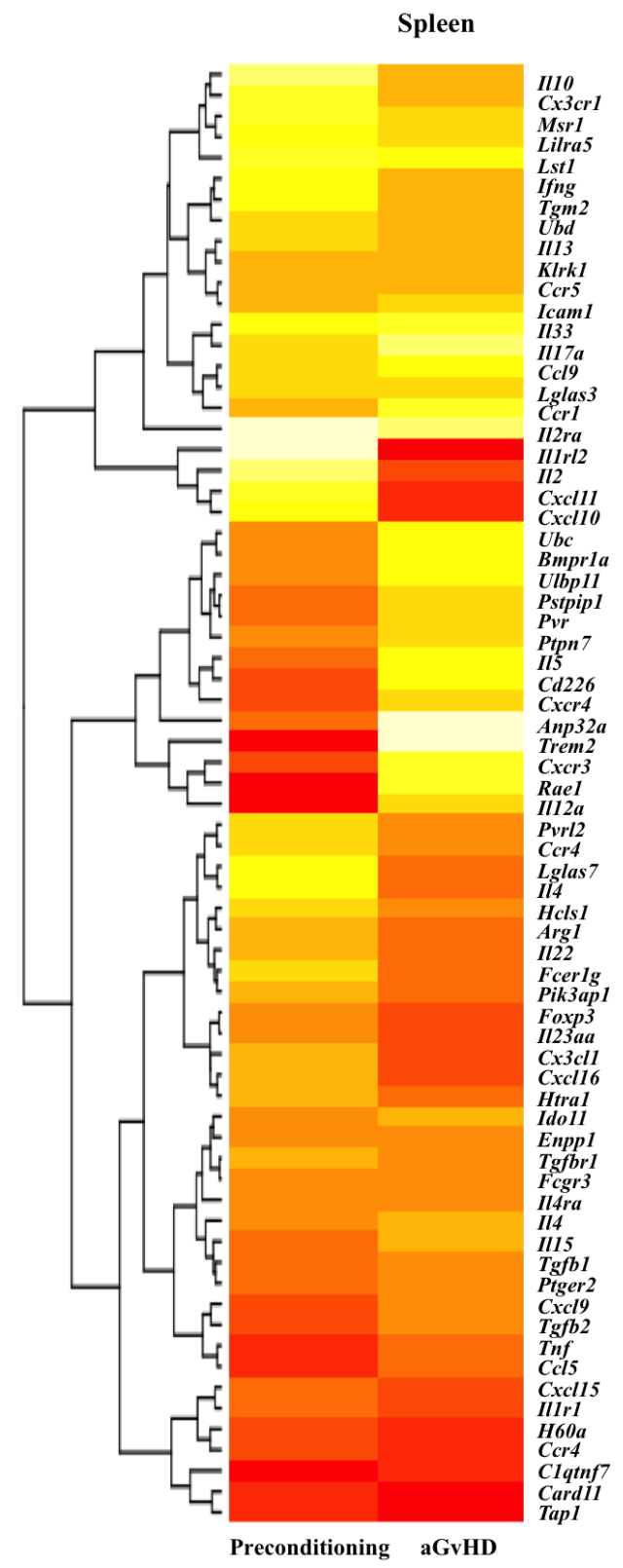


$\mathrm{E}$

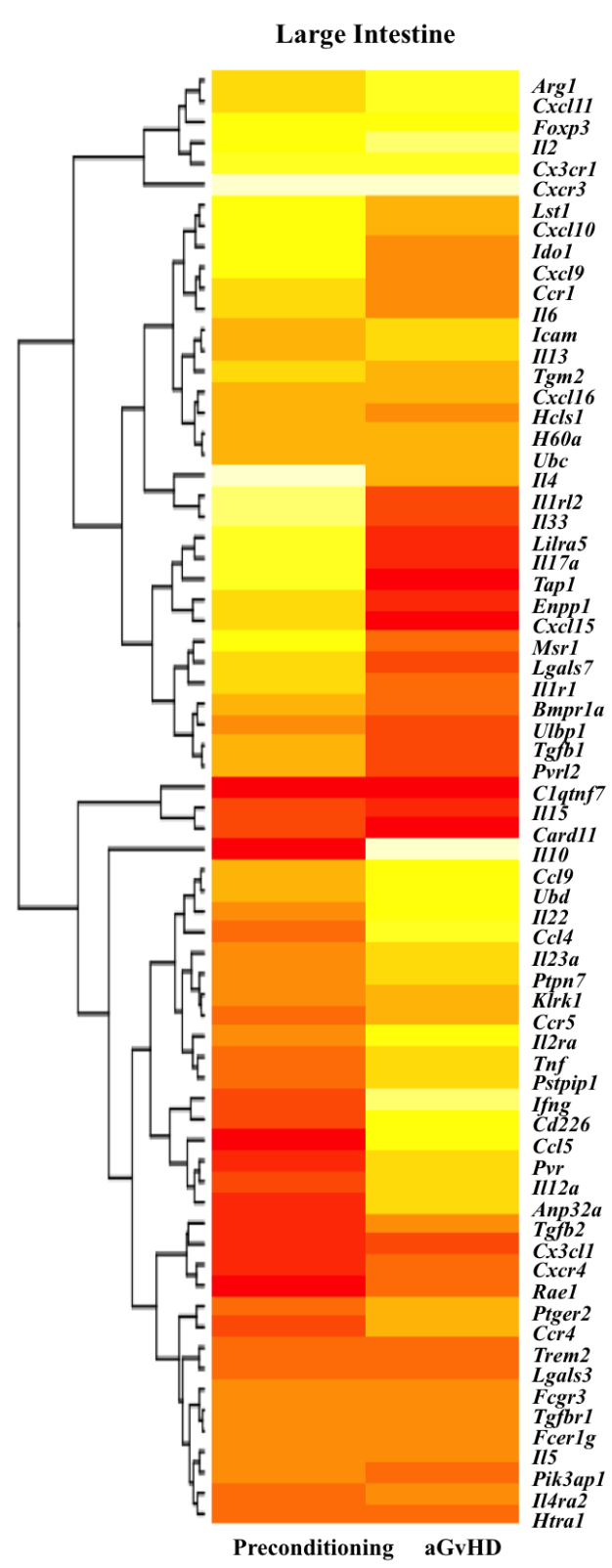

$\mathrm{F}$

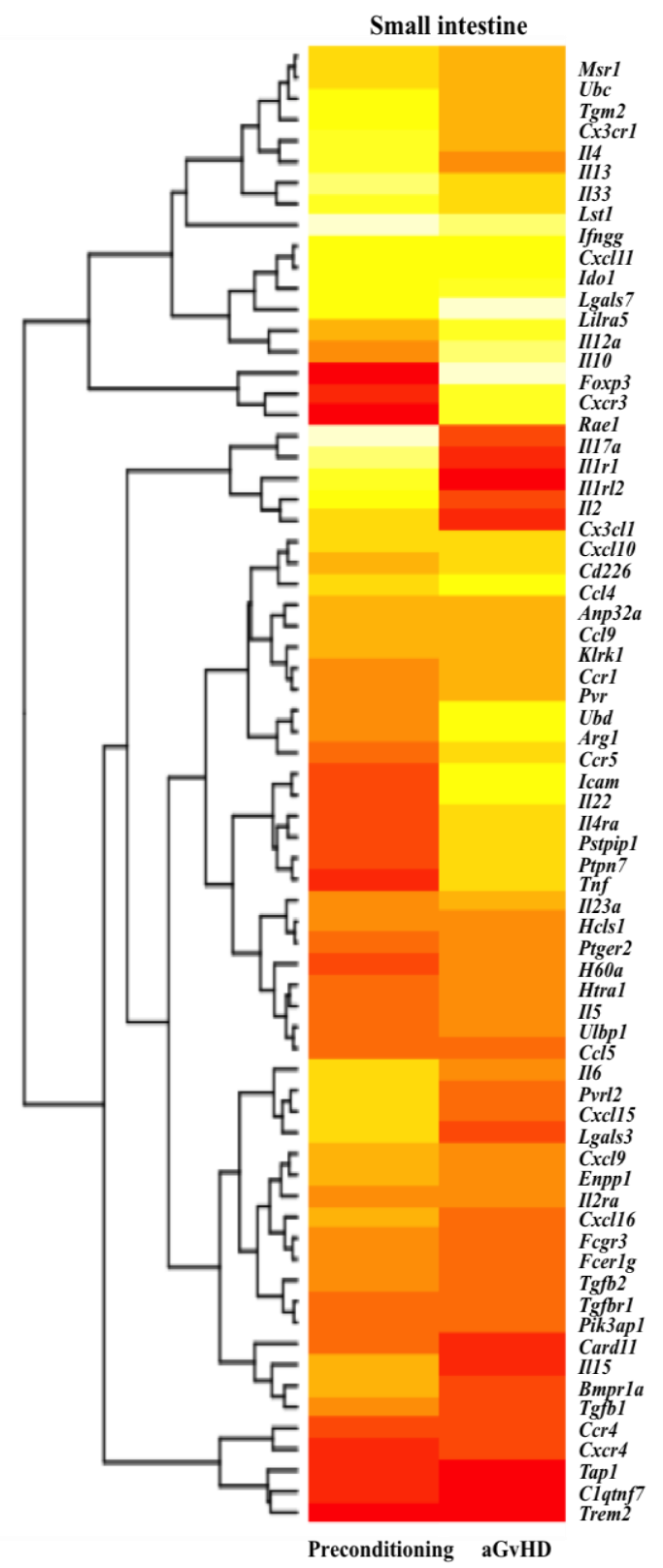

Figure 4.3: Hierarchical clustering of mRNA expression of genes in mouse aGvHD compared to preconditioning, in different organs.

Hierarchical cluster of genes in the mouse during aGvHD and preconditioning in the different organs, $\mathbf{A}$. Liver B. Lung C. Skin D. Spleen E. Small intestine F. Large intestine. The x-axis represents cluster of gene expression patterns in different tissues. The y-axis represents clusters of regulated genes during aGvHD. The color key represents the scaled fold changes from -2 (downregulated) to +2 (upregulated). All fold change values are shown (even those with $\mathrm{p}$-value $=\mathrm{ns}$ ) 


\subsection{Comparison of gene expression in different target organs in different species}

\subsubsection{Comparison of gene expression between mouse and rat aGvHD}

First, we compared the gene expression profiles of the previously identified candidate genes in the mouse and rat tissues (Figure 4.4). The expression of genes was very similar in the lung ( $\mathrm{p}=0.02$, $\left.\mathrm{R}^{2}=0.349\right)$, skin $\left(\mathrm{p}=0.0122, \mathrm{R}^{2}=0.394\right)$ and the small intestine $\left(\mathrm{p}=0.0122, \mathrm{R}^{2}=0.394\right)$. In contrast, the genes are differentially expressed in the liver $\left(\mathrm{p}=0.78, \mathrm{R}^{2}=0.007\right)$. The similarities in gene expression between the mouse and rat models are significant as the aGvHD models that we used for the mouse and rat were different. The aGvHD mouse model is fully mismatched and leads to a more severe aGvHD response compared to the rat MHC congenic model that is only MHC mismatched and aGvHD response is less severe, therefore we expected a more profound regulation of genes in the mouse. This was in line with our results, whereby, several genes that were significantly regulated in the mouse showed a similar trend in expression in the rat but were not necessarily significantly regulated.

In the liver, we observed an increased regulation in the candidate genes regulated by IFN- $\gamma$ in both the mouse and rat. In contrast, genes associated with $\mathrm{T}$ and $\mathrm{B}$ cell activation showed a trend of downregulation in the rat but were upregulated in the mouse liver. Moreover, Htral and Clqtnf7, which are significantly increased in the mouse liver but reduced in the rat. In contrast, the other three tissues showed a significant correlation in the gene expression patterns between the two species. Overall, the liver seems to follow a significantly different pattern of gene expression between the different species, as well as to other target organs of the same species.

In all the tissues, we observed an increased trend of regulation in the mRNA expression of genes such as Ubd, Lstl and Lilra5. However several genes have a mixed gene expression pattern in the different species. 


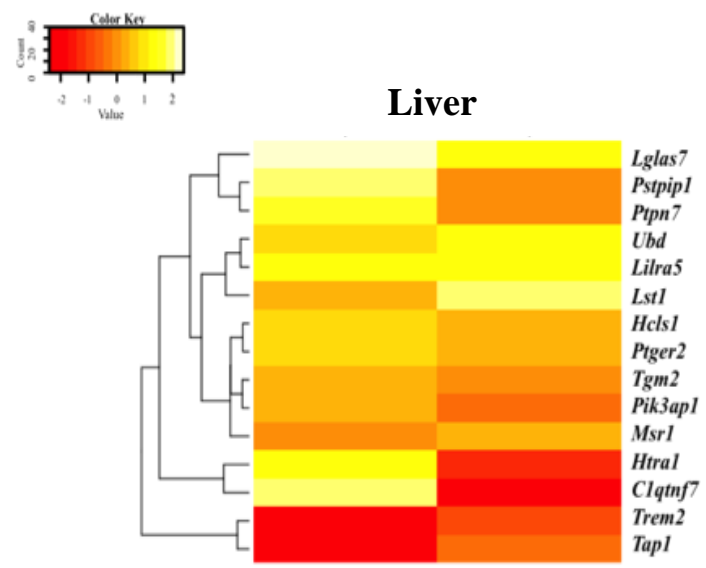

Mouse Rat

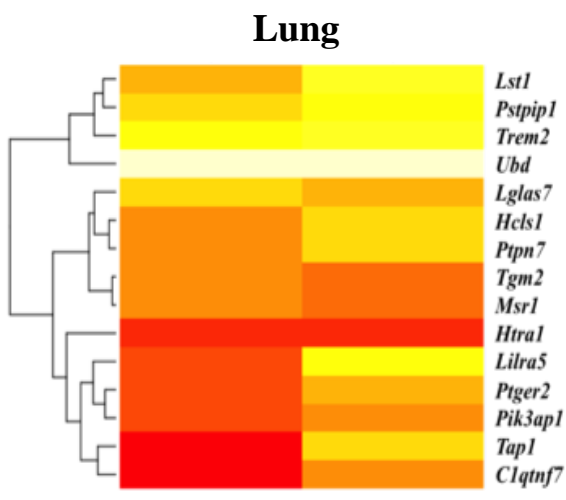

Mouse
Rat
Skin

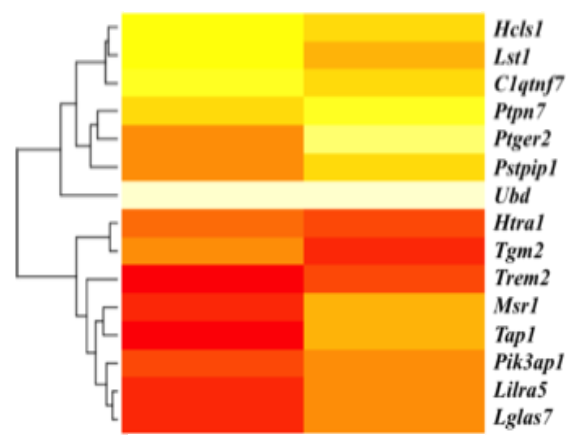

$H c l s I$

Clqmf?

Prpn 7

Iral

rem2

Lilras

\section{Mouse Rat \\ Rat different organs.}

Figure 4.4: Hierarchical clustering of mRNA expression of genes between mouse and rats in

A. Hierarchical cluster of genes in the mouse and rat liver during aGvHD B. Hierarchical cluster of genes in the mouse and rat lung during aGvHD C. Hierarchical cluster of genes in the mouse and rat skin during aGvHD D. Hierarchical cluster of genes in the mouse and rat small intestine during aGvHD. The $x$-axis represents cluster of gene expression patterns in different tissues. The y-axis represents clusters of regulated genes during aGvHD. The color key represents the scaled fold changes from -2 (downregulated) to +2 (upregulated). All fold change values are shown (even those with $\mathrm{p}$-value $=\mathrm{ns}$ ) 


\subsubsection{Comparison of gene expression between mouse intestinal and human GI GvHD}
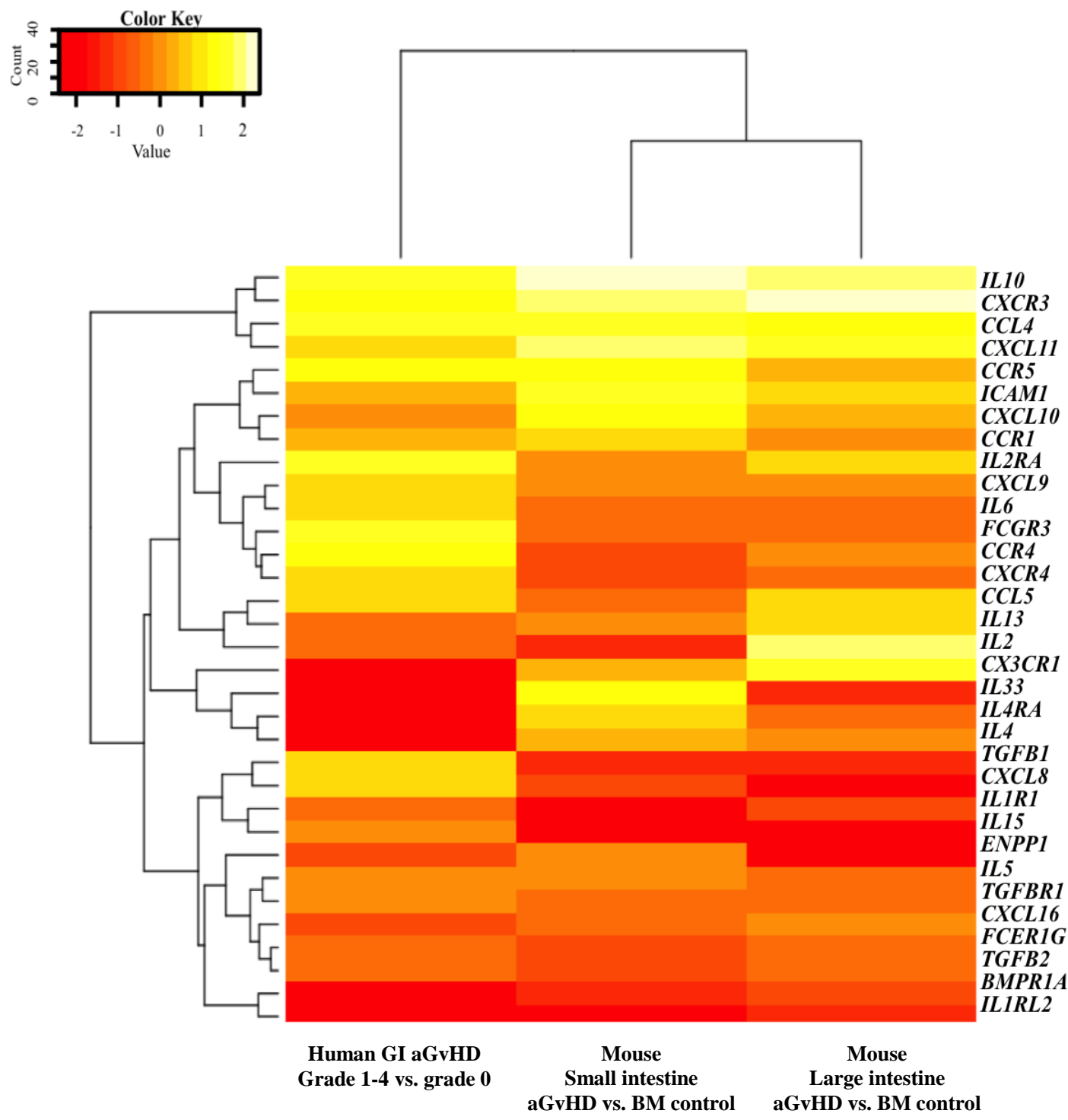

Figure 4.5: Hierarchical clustering of mRNA expression of genes between human GI GvHD (histological grades 1-4) compared to patients with no GvHD (grade 0) and mouse intestinal aGvHD.

Hierarchical cluster of genes in the small and large intestine in aGvHD mouse and GI aGvHD human biopsies. The $\mathrm{x}$-axis represents cluster of gene expression patterns in different tissues. The $\mathrm{y}$-axis represents clusters of regulated genes during aGvHD. The color key represents the scaled fold changes from -2 (downregulated) to +2 (upregulated). All fold change values are shown (even those with $\mathrm{p}$-value $=\mathrm{ns}$ ) 
The gene expression profile of GI aGvHD biopsies compared to either aGvHD-affected mouse small or large intestines was not very similar. However, a large proportion of genes were regulated in the same direction. Based on the hierarchical grouping of genes, two groups were separated. One population of genes were increased, mainly involving Th1 chemokines, whereas the other group was downregulated, mainly involving Th2 cytokines (Figure 4.5). Overall, the aGvHD in both the human GI aGvHD biopsies, as well as in the small and large intestines, was strongly associated with a chemokine-mediated aGvHD response. There was a strong upregulation of $C X C R 3$, and its ligands, CXCL9, CXCL1O and CXCL11. Other chemokines such as CCL5, and chemokine receptors, CCR1 and CCR5 were increased too. The chemokines CXCL8 (CXCl15), CXCL16, and other cytokines, mostly associated with Th2 responses, such as IL4, ILAR, IL33 and IL5 were decreased.

\subsubsection{Comparison of gene expression between mouse, rat aGvHD in small intestine and human GI GvHD}

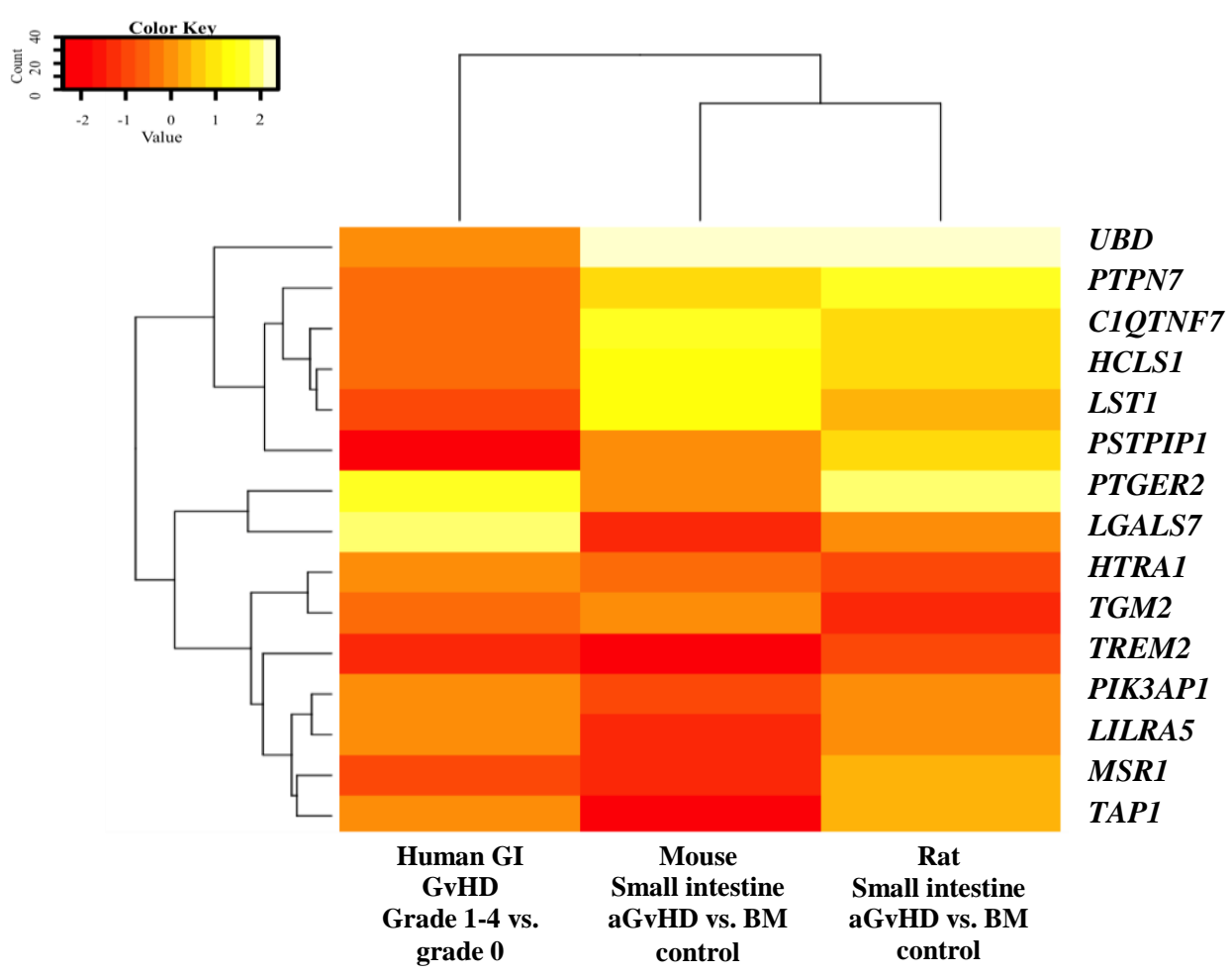

Figure 4.6: Hierarchical clustering of mRNA expression of candidate genes between mice, rat and human GI aGvHD small intestine.

Hierarchical cluster of genes in the mice, rat and human GI aGvHD small intestines. The x-axis represents cluster of gene expression patterns in different tissues. The y-axis represents clusters of regulated genes 
during aGvHD. The color key represents the scaled fold changes from -2 (downregulated) to +2 (upregulated). All fold change values are shown (even those with $p$-value $=n s$ )

Of the candidate genes, we observed a correlation between the gene expression in the aGvHD mouse and rat small intestine, but it did not significantly correlate to the mRNA expression in the GI aGvHD biopsies. However, several genes were similar expressed in all three species, such as HTRA1, TGM2, TREM2, TAP1, MSR1, LILRA5 and PIK3AP1. Most of the genes similarly regulated were either regulated by IFN- $\gamma$ (Figure 4.6).

The variations of the gene regulation patterns observed in this study could be due a number of reasons. The human aGvHD is completely different to the aGvHD animal models in terms of treatment before and after transplant, the timing of the transplant and heterogeneity of the sample population. In addition, pre-existing conditions prior to transplant, and infections such as CMV might also significantly alter the regulation patterns of the genes. Nevertheless, several genes were successfully validated to be regulated in the same manner in all the three species.

Also, important species differences need to be considered when comparing gene expression in multiple species (Mestas and Hughes, 2004). For example, marked differences in the anatomy, physiology, metabolism, pharmacology and microbiota composition play an important role in the pathophysiology of aGvHD (Schroeder and DiPersio, 2011). In addition, age plays an important role in influencing the efficacy of the immune reconstitution post transplant, effects of long-term therapy, as well as susceptibility to GvHD (Ordemann et al., 2002). Opportunistic infections are also important in affecting transplant outcome that are not modeled in rodents kept in specific-pathogenfree (SPF) conditions.

Another important difference in gene expression could be a result of the homogenous genetic composition of inbred rodents, in contrast to the heterogeneity in humans (Schroeder and DiPersio, 2011). Moreover, the immune cell populations vary in the different species. The T cell expansion is also homogeneous in rodents, contrary to the heterogeneous $\mathrm{T}$ cell response in humans (Seok et al., 2013). The proportion of lymphocyte subsets, such as $\mathrm{CD} 4^{+}, \mathrm{CD} 8^{+}$and Tregs, between the different species can remarkably influence the aGvHD pathophysiology (Schroeder and DiPersio, 2011). In addition, the Th1 and Th2 paradigm is of concern, as the polarization is relatively easy to observe in rodents, however it is more difficult to make clear distinctions in humans, as both $\mathrm{T}$ cell types are typically generated simultaneously (Allen and Maizels, 1997; Gor et al., 2003). In addition, differences have emerged between chemokines and their receptors in human and murine systems, which need to be considered when comparing gene expression patterns between humans and rodents. 
Moreover human endothelial cells constitutively express both MHC class I and II molecules, whereas murine endothelial cells only express MHC I class molecules (Choo et al., 1997). Furthermore, human endothelial cells can present antigen to resting memory $\mathrm{CD}^{+}$and $\mathrm{CD}^{+} \mathrm{T}$ cells (Mestas and Hughes, 2001; Murphy et al., 1999; Pober JS, Kluger MS, 2001), whereas mouse endothelial cells can only activate $\mathrm{CD}^{+} \mathrm{T}$ cells (Kreisel et al., 2002), but not $\mathrm{CD} 4^{+} \mathrm{T}$ cells.

It is well known that conditioning prior to HSCT causes tissue damage and induction of proinflammatory responses that affect the GvHD outcome (Gendelman et al., 2004; Mabed et al., 2005). Therefore, the time of transplant and different conditioning regimens significantly affect the transplant outcome (Schwarte and Hoffmann, 2005). Murine models frequently involve TBI as the conditioning, as in our aGvHD model, whereas in the clinical setting, patients are usually subjected to chemotherapy, and only a few patients receive TBI (Hülsdünker and Zeiser, 2015). Also, it is known that steroids affect gene regulation, which would explain some of the difference in gene expression in the human GI aGvHD biopsies.

The extent of MHC mismatch also plays a role in observed variations of gene expression. For example, in our study, our mouse aGvHD model $\left(\mathrm{C} 57 \mathrm{BL} / 6\left(\mathrm{H} 2^{\mathrm{b}}\right) \rightarrow \mathrm{BALB} / \mathrm{c}\left(\mathrm{H} 2^{\mathrm{d}}\right)\right)$ is mismatched for MHC and mHags, whereas our rat aGvHD model (PVG.7B $\left(\mathrm{RT}^{\mathrm{c}}\right) \rightarrow$ PVG.1N $\left(\mathrm{RT}^{\mathrm{n}}\right)$ ) is only MHC-mismatched. On the other hand, patients that eventually develop aGvHD, are differently well matched for HLA prior to transplant.

Taken together, these differences could explain the variations in the gene expression patterns between our different rodent models compared to human GI aGvHD.

\subsection{Gene expression pattern of KLRK1 and its ligands in aGvHD tissues in different species}

Considering the presence of several ligands for NKG2D, it is likely that the ligands are regulated differently via stress pathways. Presumably, the regulation of distinct ligands, as a response to cells undergoing different types of stress, could allow the receptor to stimulate a response in different contexts. The diversity in ligands makes immune evasion by viruses that inhibit or destroy the ligands, more difficult. The affinity of the ligands to the receptor could alter their function on the receptor under different conditions, or the way they are secreted or shed from cells. Moreover, ligands could exert different effects on different cells that express them, even in the absence of engagement of the NKG2D receptor (Raulet et al., 2013). It was also suggested that the ligands 
could engage other receptors aside from the NKG2D making it possible for them to have a wide range of functions (Kriegeskorte et al., 2005).

The functional interaction of several SNPs within the MICA gene could explain the varying expression intensity of MICA for different MICA alleles (Isernhagen et al., 2015, 2016a; Shafi et al., 2011). The relevance of NKG2D signaling for HSCT outcome was further shown in an aGvHD mouse model that developed an ameliorated aGvHD response on transient blockade of NKG2D on donor $\mathrm{CD}^{+} \mathrm{T}$ cells after transplantation (Karimi et al. 2015). Furthermore, clinical studies demonstrated the effects of RAET1L genotypes, encoding the NKG2D ligand, ULBP6 and the NKG2D encoding gene KLRK1 (Espinoza et al., 2009) and MICA genotypes (Isernhagen et al., 2015, 2016a) on overall survival of patients post transplant.

We observed a significantly differential regulation of both NKG2D and its ligands in different aGvHD target organs, i.e., the liver, lung, skin, small and large intestines, as well as the non-target organ spleen. Moreover, the Klrkl expression were significantly regulated due to preconditioning in the BM control mouse compared to the healthy controls, and its expression was further in mouse with aGvHD, suggesting that conditioning regimens that reduce induction of these molecules, could ameliorate aGvHD. The expression of the KLRK1 gene was increased in all the tissues in the mouse and rat, except in the rat it was decreased in the small intestine, as well as in the human GI aGvHD clinical biopsies, although it did not reach statistical significance in the rat or human biopsies. This could indicate an infiltration of NKG2D expressing lymphocytes in the different organs, or activation of alloreactive donor $\mathrm{T}$ cells in the organs.

The NKG2D ligands, on the other hand, had a more diverse pattern of expression in the different tissues. Rael mRNA was significantly reduced in aGvHD mouse in all the tissues. Interestingly, it was possible to clearly differentiate the regulation of Rael in the BM control mouse due to preconditioning, as well as the aGvHD mouse compared to the controls. The expression of the gene was reduced significantly as a result of preconditioning, after which its expression increased in the small intestine and spleen as a result of aGvHD, which could be explained by increased tissue injury to these organs, since an increase in RAE-1 protein expression has been shown to be associated with increase in aGvHD and tissue damage in the colon of mouse after transplant (Karimi et al. 2015). The overall expression of Rael, however, was downregulated in the aGvHD tissues compared to the healthy controls. On the other hand, H60a showed a more mixed expression pattern in the different aGvHD tissues compared to the healthy controls. On the other hand, Ulbp1 mRNA was significantly increased in the different aGvHD mouse tissues compared to the healthy controls. In the rat, we observed a significant increase in the expression of Raell mRNA in the liver, but reduced significantly in the skin. Similarly, Rrlt was also increased in the liver, but did not reach statistical significance. In the human GI aGvHD biopsies, based on the clinical score we observed a significant 
upregulation of $U L B P 1$ and a downregulation of $M I C B$. Additionally, $U L B P 3$ was significantly increased in severe (grade 2-4) aGvHD compared to low or no aGvHD (grade 0-1). Also, the expression of MICA was higher in patients who died due to transplant related causes compared to patients who died due to unrelated causes or patients that are still alive. In addition, patients who were treated with steroids had a higher mRNA expression of MICA and lower expression of ULBP1, than patients who were not given steroids. Moreover, the KLRKI and MICA mRNA expression was significantly dependent on the EBMT risk score, suggesting that an increased expression of both the genes was associated with an increased risk of aGvHD incidence.

\subsection{Gene expression pattern of $C D 226$ and its ligands in aGvHD tissues in different species}

DNAM-1 is another activating NK receptor, which also serves as a costimulatory molecule on CD8 ${ }^{+}$ T cells (Zingoni et al., 2012). DNAM-1 or CD226 is expressed on NK cells and CD8 ${ }^{+} \mathrm{T}$ cells in humans (Shibuya et al., 1996), as well as $\mathrm{CD}^{+}{ }^{+} \mathrm{T}$ cells and monocytes whereas in mouse DNAM-1 is expressed by only approximately $40-50 \% \mathrm{NK}$ cells, all $\mathrm{CD}^{+} \mathrm{T}$ cells and activated $\mathrm{CD} 4^{+} \mathrm{T}$ cells (Dardalhon et al., 2005). DNAM-1 binds to its ligands CD112 encoded by poliovirus receptor 2 (PVRL2), expressed on epithelial cells, and CD155 encoded by $P V R$ expressed on epithelial cells, endothelial cells and APCs (Bottino et al., 2003; Tahara-Hanaoka et al., 2004). Blockade or deficiency of either DNAM-1 on donor cells, has been shown to reduce the intensity of acute graft versus host disease (aGvHD) in mouse (Nabekura et al., 2010).

We observed an elevated expression of CD226 mRNA in all the mouse aGvHD tissues compared to healthy controls. Similarly, an increase in gene expression of its ligands $P v r$ in the mouse and Pvrl 2 in mouse and rat, across the different aGvHD tissues was observed. In the human GI aGvHD biopsies, we saw a similar increase in the expression of CD226 and its ligands, but the regulation was not statistically significant. Moreover, patients who died due to transplant related causes had a lower expression of $P V R L 2$ compared to other patients who were still alive or died due to unrelated causes. In addition, both $C D 226$ and $P V R$ were decreased in patients who were treated with steroids compared to patients who were not given any steroids. Overall, an elevated expression of $C D 226$ and its ligands was observed in the different aGvHD tissues. DNAM-1 expressing T or NK cell infiltration in the different tissues is the most likely explanation for this expression pattern. Another explanation could be the activation of alloreactive $\mathrm{T}$ cells in the peripheral organs, leading to an increase in the expression of these genes. 


\subsection{Gene expression in human GI aGvHD biopsies}

We found several genes that were differentially regulated between patients with and without steroids. Interestingly, the presence of steroid treatment in patients significantly altered the gene expression in patients with aGvHD compared to controls. In the overall patient cohort, the clinical patient aGvHD scores (1-4) were associated with an increased expression of HCLS1, VCAM1, CCR5, CXCR3, CCR1, IL2 and IL6, compared to patients with no aGvHD (grade 0). The genes that were associated with an increase in clinical symptoms of aGvHD in patients were mostly chemokines and genes associated with activation of $\mathrm{B}$ and $\mathrm{T}$ cells. This is in line with $\mathrm{T}$ cell infiltration in the skin and gut, which in turn leads to heightened clinical aGvHD symptoms prior to taking a biopsy of the gut. Interestingly, the gene regulation patterns due to histological and clinical scores were very different. In biopsies with histological aGvHD grades 1-4, compared to no aGvHD (grade 0), we observed an upregulation of IL2RA (CD25), which is present on activated T cells and Tregs. This suggests a strong infiltration of $\mathrm{T}$ cells in the patient GI biopsies, as well as heightened alloreactive T cell activation. On further grouping the patient biopsies based on grade 2-4 vs. grade $0-1$, we could identify several genes that were differentially regulated in the severe aGvHD biopsies compared to patients with low or no aGvHD. We observed a more chemokine-based regulation of genes, whereby CXCL16 and CX3CR1 were significantly upregulated, whereas CXCL8 and CCL4 were significantly downregulated. Interestingly, this pattern of expression was the similar to mouse, where the expression of $C x c l 16$ and $C x 3 c r l$ was upregulated in the intestines whereas $C x c l 15$, a homolog to CXCL8 and Ccl4 was significantly decreased in the intestine. Furthermore, we found IL33 to be increased in patients that died due to TRM compared to patients who were still alive or died due to unrelated causes.

It is possible that several of the genes were significantly regulated due to the administration of steroids, instead of aGvHD. To better separate the changes in the gene expression patterns in the biopsies due to aGvHD, we separated the patients who did not receive steroids, from the entire patient group. In the patient group without steroids, several genes were still regulated in the same manner, whereas certain genes were additionally regulated, for example PSTPIP1 was significantly downregulated in patients with severe aGvHD (grade 2-4) compared to grade 0-1.

Taken together, the gene expression data from patient biopsies is of crucial importance. Identifying genes that are similarly expressed in all three species would be of more significance. Genes such as $U B D$, that are upregulated in all the different tissues in all the species, could be an important target to better understand mechanisms of aGvHD and possibly a therapeutic target. Further functional studies using knockout rodent models of aGvHD could be the next step in elucidating the importance of the function of these genes during aGvHD. 


\section{Summary and conclusions}

Several studies focussing on mRNA expression profiling in aGvHD tissues have been carried out in the past. Such mRNA profiling studies are important in understanding the pathophysiology of aGvHD in the different target organs affected by aGvHD. While most studies have been carried out in 1 to 2 tissues, we had the unique opportunity to study the mRNA expression profiles of several focus genes in different target organs of aGvHD, and in different species simultaneously. We showed that in mice, aGvHD was associated with an increase in the Th1, Th2 and Th17 responses and the regulation of these genes was more pronounced in the lung and intestinal biopsies. Moreover, genes associated with Treg regulation and activation were overexpressed in the different target organs, most notably in the intestinal biopsies. These results give us an idea on lymphocyte migration patterns in the different target tissues during aGvHD.

In all the aGvHD target tissues, we observed a pronounced chemokine response to aGvHD. A similar chemokine response was observed in the human GI aGvHD biopsies. Several candidate genes, mainly associated with $\mathrm{T}$ and $\mathrm{B}$ cell activation, and inflammatory responses were also increased in the mouse tissues and showed a similar trend of regulation in the rat and human aGvHD biopsies as well. Notably, a number of genes were significantly altered as a result of preconditioning alone, and their expression was further regulated due to aGvHD. Furthermore, the gene expression patterns in the target organs of aGvHD, such as intestine and liver, were significantly different from the non-classical aGvHD target organs, such as spleen.

We also found an increased trend in expression of KLRK1, encoding NKG2D and CD226, encoding DNAM-1 in all the mice and rat tissues, however in the human biopsies KLRK1 mRNA showed a trend of upregulation, whereas $C D 226$ remained unchanged. The expression patterns of their ligands were most interesting. On one hand, mRNA expression of the NKG2D ligand Ulbpl was upregulated in the different mouse tissues, whereas $H 60 a$ was upregulated in the intestinal biopsies, whereas downregulated in the skin, and Rael was significantly downregulated in the different mouse aGvHD tissues. Similarly, Raell was upregulated in the aGvHD rat liver, whereas it was downregulated in the skin. On the other hand, the expression of most of these genes were not changed during human GI aGvHD, however $M I C B$ was significantly downregulated, whereas we observed a trend of upregulation of MICA. The expression patterns of the DNAM-1 ligands were more similar between the different species. In the mice, both $P v r$ and $P v r l 2$ were significantly increased, and in the rats they showed a trend of upregulation. On the other hand, in the human GI biopsies, they were not significantly altered.

Moreover, an increased expression of $M I C A$, and decreased $P V R L 2$ expression was associated with TRM in patients. Similarly, MICA was upregulated in patients that were treated with high doses of 
steroids, whereas the expression of ULBP1,CD226 and PVR was downregulated in these steroidtreated patients. The regulation of several of these genes in mice due to preconditioning and transplant, suggests that preconditioning, significantly alters the expression of KLRK1, CD226 and their ligands during aGvHD, suggesting that conditioning regimens which lead to less induction of these ligands might ameliorate the risk of aGVHD after HSCT. 


\section{References:}

Aggarwal, S., Ghilardi, N., Xie, M.-H., de Sauvage, F.J., and Gurney, A.L. (2002). Interleukin-23 Promotes a Distinct CD4 T Cell Activation State Characterized by the Production of Interleukin17. J. Biol. Chem. 278, 1910-1914.

Ahmed, S.S., Wang, X.N., Norden, J., Pearce, K., El-Gezawy, E., Atarod, S., Hromadnikova, I., Collin, M., Holler, E., and Dickinson, A.M. (2015). Identification and validation of biomarkers associated with acute and chronic graft versus host disease. Bone Marrow Transplant. 50, 15631571.

Alegre, M.L., Frauwirth, K. a, and Thompson, C.B. (2001). T-cell regulation by CD28 and CTLA4. Nat. Rev. Immunol. 1, 220-228.

Allen, J.E., and Maizels, R.M. (1997). Th1-Th2: Reliable paradigm or dangerous dogma? Immunol. Today 18, 387-392.

Allen, J.L., Fore, M.S., Wooten, J., Roehrs, P.A., Bhuiya, N.S., Hoffert, T., Sharf, A., Deal, A.M., Armistead, P., Coghill, J., et al. (2012). B cells from patients with chronic GVHD are activated and primed for survival via BAFF-mediated pathways. Blood 120, 2529-2536.

Antin, J.H., and Ferrara, J.L. (1992). Cytokine dysregulation and acute graft-versus-host disease. Blood 80, 2964-2968.

Antin, J.H., Lee, S.J., Neuberg, D., Alyea, E., Soiffer, R.J., Sonis, S., and Ferrara, J.L.M. (2002). A phase I/II double-blind, placebo-controlled study of recombinant human interleukin-11 for mucositis and acute GVHD prevention in allogeneic stem cell transplantation. Bone Marrow Transplant. 29, 373-377.

Appelbaum, F.R. (2001). Haematopoietic cell transplantation as immunotherapy. Nature 411, 385389.

Atkinson, K., Horowitz, M.M., Gale, R.P., van Bekkum, D.W., Gluckman, E., Good, R.A., Jacobsen, N., Kolb, H.J., Rimm, A.A., and Ringdén, O. (1990). Risk factors for chronic graftversus-host disease after HLA-identical sibling bone marrow transplantation. Blood 75, 24592464.

Atkinson, K., Matias, C., Guiffre, A., Seymour, R., Cooley, M., Biggs, J., Munro, V., and Gillis, S. (1991). In vivo administration of granulocyte colony-stimulating factor (G-CSF), granulocytemacrophage CSF, interleukin-1 (IL-1), and IL-4, alone and in combination, after allogeneic murine hematopoietic stem cell transplantation. Blood 77, 1376-1382.

Bacigalupo, A., Ballen, K., Rizzo, D., Giralt, S., Lazarus, H., Ho, V., Apperley, J., Slavin, S., Pasquini, M., Sandmaier, B.M., et al. (2009). Defining the Intensity of Conditioning Regimens: Working Definitions. Biol. Blood Marrow Transplant. 15, 1628-1633.

de Baey, a, Fellerhoff, B., Maier, S., Martinozzi, S., Weidle, U., and Weiss, E.H. (1997). Complex expression pattern of the TNF region gene LST1 through differential regulation, initiation, and alternative splicing. Genomics 45, 591-600.

Baggiolini, M. (1998). Chemokines and leukocyte traffic. Nature 392, 565-568.

Barner, M., Mohrs, M., Brombacher, F., and Kopf, M. (1998). Differences between IL-4R alphadeficient and IL-4-deficient mice reveal a role for IL-13 in the regulation of Th2 responses. Curr. 
Biol. 8, 669-672.

BARNES, D.W., CORP, M.J., LOUTIT, J.F., and NEAL, F.E. (1956). Treatment of murine leukaemia with $\mathrm{X}$ rays and homologous bone marrow; preliminary communication. Br. Med. J. 2, $626-627$.

Bauer, S., Groh, V., Wu, J., Steinle, a, Phillips, J.H., Lanier, L.L., and Spies, T. (1999). Activation of NK cells and T cells by NKG2D, a receptor for stress-inducible MICA. Science 285, 727-729.

Beck, S., and Trowsdale, J. (2000). The human major histocompatability complex: lessons from the DNA sequence. Annu. Rev. Genomics Hum. Genet. 1, 117-137.

Bensinger, W.I., Buckner, C.D., Shannon-Dorcy, K., Rowley, S., Appelbaum, F.R., Benyunes, M., Clift, R., Martin, P., Demirer, T., Storb, R., et al. (1996). Transplantation of allogeneic CD34+ peripheral blood stem cells in patients with advanced hematologic malignancy. Blood 88,4132 4138.

Bensinger, W.I., Martin, P.J., Storer, B., Clift, R., Forman, S.J., Negrin, R., Kashyap, A., Flowers, M.E., Lilleby, K., Chauncey, T.R., et al. (2001). Transplantation of bone marrow as compared with peripheral-blood cells from HLA-identical relatives in patients with hematologic cancers. N. Engl. J. Med. 344, 175-181.

Beres, A.J., and Drobyski, W.R. (2013). The role of regulatory T cells in the biology of graft versus host disease. Front. Immunol. 4, 163.

Von Bergwelt-Baildon, M.S., Vonderheide, R.H., Maecker, B., Hirano, N., Anderson, K.S., Butler, M.O., Xia, Z., Zeng, W.Y., Wucherpfennig, K.W., Nadler, L.M., et al. (2002). Human primary and memory cytotoxic $\mathrm{T}$ lymphocyte responses are efficiently induced by means of CD40-activated $\mathrm{B}$ cells as antigen-presenting cells: Potential for clinical application. Blood 99, 3319-3325.

Bettelli, E., Carrier, Y., Gao, W., Korn, T., Strom, T.B., Oukka, M., Weiner, H.L., and Kuchroo, V.K. (2006). Reciprocal developmental pathways for the generation of pathogenic effector TH17 and regulatory T cells. Nature 441, 235-238.

Billingham, R.E. The biology of graft-versus-host reactions. Harvey Lect. 62, 21-78.

Blaise, D., Olive, D., Hirn, M., Viens, P., Lafage, M., Attal, M., Stoppa, A.M., Gabert, J., Gastaut, J.A., and Camerlo, J. (1991). Prevention of acute GVHD by in vivo use of anti-interleukin-2 receptor monoclonal antibody (33B3.1): a feasibility trial in 15 patients. Bone Marrow Transplant. $8,105-111$.

Blaser, B.W., Roychowdhury, S., Kim, D.J., Schwind, N.R., Bhatt, D., Yuan, W., Kusewitt, D.F., Ferketich, A.K., Caligiuri, M.A., and Guimond, M. (2005). Donor-derived IL-15 is critical for acute allogeneic graft-versus-host disease. Blood 105, 894-901.

Bottino, C., Castriconi, R., Pende, D., Rivera, P., Nanni, M., Carnemolla, B., Cantoni, C., Grassi, J., Marcenaro, S., Reymond, N., et al. (2003). Identification of PVR (CD155) and Nectin-2 (CD112) as cell surface ligands for the human DNAM-1 (CD226) activating molecule. J. Exp. Med. 198, 557-567.

Bouazzaoui, A., Spacenko, E., Mueller, G., Miklos, S., Huber, E., Holler, E., Andreesen, R., and Hildebrandt, G.C. (2009). Chemokine and chemokine receptor expression analysis in target organs of acute graft-versus-host disease. Genes Immun. 10, 687-701.

Braun, M.Y., Lowin, B., French, L., Acha-Orbea, H., and Tschopp, J. (1996). Cytotoxic T cells 
deficient in both functional fas ligand and perforin show residual cytolytic activity yet lose their capacity to induce lethal acute graft-versus-host disease. J. Exp. Med. 183, 657-661.

Brenner, S., Whiting-Theobald, N., Kawai, T., Linton, G.F., Rudikoff, A.G., Choi, U., Ryser, M.F., Murphy, P.M., Sechler, J.M.G., and Malech, H.L. (2004). CXCR4-transgene expression significantly improves marrow engraftment of cultured hematopoietic stem cells. Stem Cells 22, $1128-1133$.

van Den Brink, M.R., Moore, E., Horndasch, K.J., Crawford, J.M., Hoffman, J., Murphy, G.F., and Burakoff, S.J. (2000). Fas-deficient lpr mice are more susceptible to graft-versus-host disease. J. Immunol. 164, 469-480.

Brown, D., Trowsdale, J., and Allen, R. (2004). The LILR family: modulators of innate and adaptive immune pathways in health and disease. Tissue Antigens 64, 215-225.

Buzzeo, M.P., Yang, J., Casella, G., and Reddy, V. (2008). A preliminary gene expression profile of acute graft-versus-host disease. Cell Transplant. 17, 489-494.

Campbell, J.J., Haraldsen, G., Pan, J., Rottman, J., Qin, S., Ponath, P., Andrew, D.P., Warnke, R., Ruffing, N., Kassam, N., et al. (1999). The chemokine receptor CCR4 in vascular recognition by cutaneous but not intestinal memory T cells. Nature 400, 776-780.

Carayannopoulos, L.N., Naidenko, O. V, Fremont, D.H., and Yokoyama, W.M. (2002). Cutting edge: murine UL16-binding protein-like transcript 1: a newly described transcript encoding a highaffinity ligand for murine NKG2D. J. Immunol. 169, 4079-4083.

Carlson, M.J., West, M.L., Coghill, J.M., Panoskaltsis-Mortari, A., Blazar, B.R., and Serody, J.S. (2009). In vitro-differentiated TH17 cells mediate lethal acute graft-versus-host disease with severe cutaneous and pulmonary pathologic manifestations. Blood 113, 1365-1374.

Carrier, Y., Yuan, J., Kuchroo, V.K., and Weiner, H.L. (2007). Th3 cells in peripheral tolerance. II. TGF-beta-transgenic Th3 cells rescue IL-2-deficient mice from autoimmunity. J. Immunol. 178, $172-178$.

Castor, M.G.M., Pinho, V., and Teixeira, M.M. (2012). The role of chemokines in mediating graft versus host disease: opportunities for novel therapeutics. Front. Pharmacol. 3, 23.

Cerboni, C., Fionda, C., Soriani, A., Zingoni, A., Doria, M., Cippitelli, M., and Santoni, A. (2014). The DNA Damage Response: A Common Pathway in the Regulation of NKG2D and DNAM-1 Ligand Expression in Normal, Infected, and Cancer Cells. Front. Immunol. 4.

Cerwenka, A., Baron, J.L., and Lanier, L.L. (2001). Ectopic expression of retinoic acid early inducible-1 gene (RAE-1) permits natural killer cell-mediated rejection of a MHC class I-bearing tumor in vivo. Proc. Natl. Acad. Sci. U. S. A. 98, 11521-11526.

Chen, X., Das, R., Komorowski, R., Beres, A., Hessner, M.J., Mihara, M., and Drobyski, W.R. (2009). Blockade of interleukin-6 signaling augments regulatory T-cell reconstitution and attenuates the severity of graft-versus-host disease. Blood 114, 891-900.

Choo, J.K., Seebach, J.D., Nickeleit, V., Shimizu, A., Lei, H., Sachs, D.H., and Madsen, J.C. (1997). Species differences in the expression of major histocompatibility complex class II antigens on coronary artery endothelium: implications for cell-mediated xenoreactivity. Transplantation 64, $1315-1322$.

Cobbold, S.P., Castejon, R., Adams, E., Zelenika, D., Graca, L., Humm, S., and Waldmann, H. 
(2004). Induction of foxP3+ Regulatory T Cells in the Periphery of T Cell Receptor Transgenic Mice Tolerized to Transplants. J. Immunol. 172, 6003-6010.

Constant, S., Schweitzer, N., West, J., Ranney, P., and Bottomly, K. (1995). B lymphocytes can be competent antigen-presenting cells for priming $\mathrm{CD} 4+\mathrm{T}$ cells to protein antigens in vivo. $\mathrm{J}$. Immunol. 155, 3734-3741.

Cooke, K.R., Hill, G.R., Crawford, J.M., Bungard, D., Brinson, Y.S., Delmonte, J., and Ferrara, J.L. (1998). Tumor necrosis factor- alpha production to lipopolysaccharide stimulation by donor cells predicts the severity of experimental acute graft-versus-host disease. J. Clin. Invest. 102, $1882-1891$.

Cosman, D., Müllberg, J., Sutherland, C.L., Chin, W., Armitage, R., Fanslow, W., Kubin, M., and Chalupny, N.J. (2001). ULBPs, novel MHC class I-related molecules, bind to CMV glycoprotein UL16 and stimulate NK cytotoxicity through the NKG2D receptor. Immunity 14, 123-133.

Cray, C., and Levy, R.B. (1990a). Virus-associated immune responses in mice undergoing GVHR exacerbated by concurrent MCMV infection. Transplantation 50, 1027-1032.

Cray, C., and Levy, R.B. (1990b). The presence of infectious virus but not conventional antigen can exacerbate graft-versus-host reactions. Scand. J. Immunol. 32, 177-182.

Cray, C., and Levy, R.B. (1993). CD8+ and CD4+ T cells contribute to the exacerbation of class I MHC disparate graft-vs-host reaction by concurrent murine cytomegalovirus infection. Clin. Immunol. Immunopathol. 67, 84-90.

Cuzzola, M., Fiasché, M., Iacopino, P., Messina, G., Martino, M., Console, G., Fedele, R., Massi, D., Recchia, A.G., Irrera, G., et al. (2012). A molecular and computational diagnostic approach identifies FOXP3, ICOS, CD52 and CASP1 as the most informative biomarkers in acute graftversus-host disease. Haematologica 97, 1532-1538.

Dardalhon, V., Schubart, A.S., Reddy, J., Meyers, J.H., Monney, L., Sabatos, C.A., Ahuja, R., Nguyen, K., Freeman, G.J., Greenfield, E.A., et al. (2005). CD226 is specifically expressed on the surface of Th1 cells and regulates their expansion and effector functions. J. Immunol. 175, 15581565 .

Das, H., Imoto, S., Murayama, T., Mizuno, I., Sugimoto, T., Taniguchi, R., Toda, K., Isobe, T., Nakagawa, T., Nishimura, R., et al. (2001). Kinetic analysis of cytokine gene expression in patients with GVHD after donor lymphocyte infusion. Bone Marrow Transplant. 27, 373-380.

Deeg, H.J., and Storb, R.F. (1985). Immunosuppression for marrow transplantation. Plasma Ther. Transfus. Technol. 6 .

Demetriou, M., Granovsky, M., Quaggin, S., and Dennis, J.W. (2001). Negative regulation of Tcell activation and autoimmunity by Mgat5 N-glycosylation. Nature 409, 733-739.

Dickinson, A.M., Sviland, L., Hamilton, P.J., Usher, P., Taylor, P., Jackson, G., Dunn, J., and Proctor, S.J. (1994). Cytokine involvement in predicting clinical graft-versus-host disease in allogeneic bone marrow transplant recipients. Bone Marrow Transplant. 13, 65-70.

Diefenbach, A., Hsia, J.K., Hsiung, M.-Y.B., and Raulet, D.H. (2003). A novel ligand for the NKG2D receptor activates NK cells and macrophages and induces tumor immunity. Eur. J. Immunol. 33, 381-391.

Ding, A.H., Nathan, C.F., and Stuehr, D.J. (1988). Release of reactive nitrogen intermediates and 
reactive oxygen intermediates from mouse peritoneal macrophages. Comparison of activating cytokines and evidence for independent production. J Immunol 141, 2407-2412.

Dressel, R., Dickinson, A., Rolstadt, B., and Walter, L. (2013). MHC Genes and Risk of Graft Versus Host Disease. Pat. Publ. Number 20130338035.

Duffner, U.A., Maeda, Y., Cooke, K.R., Reddy, P., Ordemann, R., Liu, C., Ferrara, J.L.M., and Teshima, T. (2004). Host dendritic cells alone are sufficient to initiate acute graft-versus-host disease. J. Immunol. 172, 7393-7398.

Ebert, L.M., Schaerli, P., and Moser, B. (2005). Chemokine-mediated control of T cell traffic in lymphoid and peripheral tissues. Mol. Immunol. 42, 799-809.

Eisenberg, S.P., Evans, R.J., Arend, W.P., Verderber, E., Brewer, M.T., Hannum, C.H., and Thompson, R.C. (1990). Primary structure and functional expression from complementary DNA of a human interleukin-1 receptor antagonist. Nature 343, 341-346.

Erhardt, A., Biburger, M., Papadopoulos, T., and Tiegs, G. (2007). IL-10, regulatory T cells, and Kupffer cells mediate tolerance in concanavalin A-induced liver injury in mice. Hepatology 45, 475-485.

Espinoza, J.L., Takami, A., Onizuka, M., Sao, H., Akiyama, H., Miyamura, K., Okamoto, S., Inoue, M., Kanda, Y., Ohtake, S., et al. (2009). NKG2D gene polymorphism has a significant impact on transplant outcomes after HLA-fully-matched unrelated bone marrow transplantation for standard risk hematologic malignancies. Haematologica 94, 1427-1434.

Ferrara, J.L., and Deeg, H.J. (1991). Graft-versus-host disease. N. Engl. J. Med. 324, 667-674.

Ferrara, J.L.M., and Reddy, P. (2006). Pathophysiology of graft-versus-host disease. Semin. Hematol. 43, 3-10.

Ferrara, J.L., Marion, A., McIntyre, J.F., Murphy, G.F., and Burakoff, S.J. (1986). Amelioration of acute graft vs host disease due to minor histocompatibility antigens by in vivo administration of anti-interleukin 2 receptor antibody. J. Immunol. 137, 1874-1877.

Ferrara, J.L.M., Levy, R., and Chao, N.J. (1999). Pathophysiologic mechanisms of acute graft-vs.host disease. Biol. Blood Marrow Transplant. 5, 347-356.

Ferrara, J.L.M., Levine, J.E., Reddy, P., and Holler, E. (2009). Graft-versus-host disease. Lancet $373,1550-1561$.

Filipovich, A.H., Weisdorf, D., Pavletic, S., Socie, G., Wingard, J.R., Lee, S.J., Martin, P., Chien, J., Przepiorka, D., Couriel, D., et al. (2005). National Institutes of Health consensus development project on criteria for clinical trials in chronic graft-versus-host disease: I. Diagnosis and staging working group report. Biol. Blood Marrow Transplant. 11, 945-956.

Flier, J., Boorsma, D.M., van Beek, P.J., Nieboer, C., Stoof, T.J., Willemze, R., and Tensen, C.P. (2001). Differential expression of CXCR3 targeting chemokines CXCL10, CXCL9, and CXCL11 in different types of skin inflammation. J. Pathol. 194, 398-405.

Fontenot, J.D., Rasmussen, J.P., Williams, L.M., Dooley, J.L., Farr, A.G., and Rudensky, A.Y. (2005). Regulatory $\mathrm{T}$ cell lineage specification by the forkhead transcription factor Foxp3. Immunity 22, 329-341.

Fukuda, T., Kitamura, D., Taniuchi, I., Maekawa, Y., Benhamou, L.E., Sarthou, P., and Watanabe, T. (1995). Restoration of surface IgM-mediated apoptosis in an anti-IgM-resistant variant of 
WEHI-231 lymphoma cells by HS1, a protein-tyrosine kinase substrate. Proc. Natl. Acad. Sci. U. S. A. $92,7302-7306$.

Fulton, W.B., Reeves, R.H., Takeya, M., and De Maio, A. (2006). A quantitative trait loci analysis to map genes involved in lipopolysaccharide-induced inflammatory response: identification of macrophage scavenger receptor 1 as a candidate gene. J. Immunol. 176, 3767-3773.

Garg, S., Boerma, M., Wang, J., Fu, Q., Loose, D.S., Kumar, K.S., and Hauer-Jensen, M. (2010). Influence of sublethal total-body irradiation on immune cell populations in the intestinal mucosa. Radiat. Res. 173, 469-478.

Gavin, M.A., Rasmussen, J.P., Fontenot, J.D., Vasta, V., Manganiello, V.C., Beavo, J.A., and Rudensky, A.Y. (2007). Foxp3-dependent programme of regulatory T-cell differentiation. Nature 445, 771-775.

Gendelman, M., Hecht, T., Logan, B., Vodanovic-Jankovic, S., Komorowski, R., and Drobyski, W.R. (2004). Host conditioning is a primary determinant in modulating the effect of IL-7 on murine graft-versus-host disease. J. Immunol. 172, 3328-3336.

Gifford, G.E., and Lohmann-Matthes, M.L. (1987). Gamma interferon priming of mouse and human macrophages for induction of tumor necrosis factor production by bacterial lipopolysaccharide. J. Natl. Cancer Inst. 78, 121-124.

Gilfillan, S., Chan, C.J., Cella, M., Haynes, N.M., Rapaport, A.S., Boles, K.S., Andrews, D.M., Smyth, M.J., and Colonna, M. (2008). DNAM-1 promotes activation of cytotoxic lymphocytes by nonprofessional antigen-presenting cells and tumors. J. Exp. Med. 205, 2965-2973.

Gluckman, E., Rocha, V., and Chastang, C. Peripheral stem cells in bone marrow transplantation. Cord blood stem cell transplantation. Baillière's Best Pract. Res. Clin. Haematol. 12, 279-292.

Goker, H., Haznedaroglu, I.C., and Chao, N.J. (2001). Acute graft-vs-host disease: pathobiology and management. Exp. Hematol. 29, 259-277.

Gooley, T.A., Chien, J.W., Pergam, S.A., Hingorani, S., Sorror, M.L., Boeckh, M., Martin, P.J., Sandmaier, B.M., Marr, K.A., Appelbaum, F.R., et al. (2010). Reduced mortality after allogeneic hematopoietic-cell transplantation. N. Engl. J. Med. 363, 2091-2101.

Gor, D.O., Rose, N.R., and Greenspan, N.S. (2003). TH1-TH2: a procrustean paradigm. Nat. Immunol. 4, 503-505.

Gratwohl, A. (2012). The EBMT risk score. Bone Marrow Transplant. 47, 749-756.

Griffin, M., Casadio, R., and Bergamini, C.M. (2002). Transglutaminases: nature's biological glues. Biochem. J. 368, 377-396.

Griffith, L.M., Pavletic, S.Z., Lee, S.J., Martin, P.J., Schultz, K.R., and Vogelsang, G.B. (2008). Chronic Graft-versus-Host Disease--implementation of the National Institutes of Health Consensus Criteria for Clinical Trials. Biol. Blood Marrow Transplant. 14, 379-384.

Groom, J.R., and Luster, A.D. (2011). CXCR3 ligands: redundant, collaborative and antagonistic functions. Immunol. Cell Biol. 89, 207-215.

Hägglund, H., Ringdén, O., Remberger, M., Lönnqvist, B., Sparrelid, E., Tammik, L., and Kumlien, G. (1998). Faster neutrophil and platelet engraftment, but no differences in acute GVHD or survival, using peripheral blood stem cells from related and unrelated donors, compared to bone marrow. Bone Marrow Transplant. 22, 131-136. 
Halliwell, B., and Gutteridge, J.M. (1992). Biologically relevant metal ion-dependent hydroxyl radical generation. An update. FEBS Lett. 307, 108-112.

Hanash, A.M., Dudakov, J.A., Hua, G., O’Connor, M.H., Young, L.F., Singer, N. V, West, M.L., Jenq, R.R., Holland, A.M., Kappel, L.W., et al. (2012). Interleukin-22 protects intestinal stem cells from immune-mediated tissue damage and regulates sensitivity to graft versus host disease. Immunity $37,339-350$.

Hannum, C.H., Wilcox, C.J., Arend, W.P., Joslin, F.G., Dripps, D.J., Heimdal, P.L., Armes, L.G., Sommer, A., Eisenberg, S.P., and Thompson, R.C. (1990). Interleukin-1 receptor antagonist activity of a human interleukin-1 inhibitor. Nature 343, 336-340.

Harrington, L.E., Hatton, R.D., Mangan, P.R., Turner, H., Murphy, T.L., Murphy, K.M., and Weaver, C.T. (2005). Interleukin 17-producing CD4+ effector T cells develop via a lineage distinct from the T helper type 1 and 2 lineages. Nat. Immunol. 6, 1123-1132.

Harris, S.G., Padilla, J., Koumas, L., Ray, D., and Phipps, R.P. (2002). Prostaglandins as modulators of immunity. Trends Immunol. 23, 144-150.

Hasegawa, H., Inoue, a, Kohno, M., Lei, J., Miyazaki, T., Yoshie, O., Nose, M., and Yasukawa, M. (2008). Therapeutic effect of CXCR3-expressing regulatory T cells on liver, lung and intestinal damages in a murine acute GVHD model. Gene Ther. 15, 171-182.

He, S., Cao, Q., Qiu, Y., Mi, J., and Zhang, J. (2008). A new approach to the blocking of alloreactive $\mathrm{T}$ cell-mediated graft-versus-host disease by in vivo administration of anti-CXCR3 neutralizing antibody. J. Immunol. 181, 7581-7592.

Heinzel, F.P., Sadick, M.D., Mutha, S.S., and Locksley, R.M. (1991). Production of interferon gamma, interleukin 2, interleukin 4, and interleukin 10 by CD4+ lymphocytes in vivo during healing and progressive murine leishmaniasis. Proc. Natl. Acad. Sci. U. S. A. 88, 7011-7015.

Highfill, S.L., Rodriguez, P.C., Zhou, Q., Goetz, C.A., Koehn, B.H., Veenstra, R., Taylor, P.A., Panoskaltsis-Mortari, A., Serody, J.S., Munn, D.H., et al. (2010). Bone marrow myeloid-derived suppressor cells (MDSCs) inhibit graft-versus-host disease (GVHD) via an arginase-1-dependent mechanism that is up-regulated by interleukin-13. Blood 116, 5738-5747.

Hill, G.R. (2009). Inflammation and Bone Marrow Transplantation. Biol. Blood Marrow Transplant. 15, 139-141.

Hill, G.R., and Ferrara, J.L. (2000). The primacy of the gastrointestinal tract as a target organ of acute graft-versus-host disease: rationale for the use of cytokine shields in allogeneic bone marrow transplantation. Blood 95, 2754-2759.

Hill, G.R., Crawford, J.M., Cooke, K.R., Brinson, Y.S., Pan, L., and Ferrara, J.L. (1997). Total body irradiation and acute graft-versus-host disease: the role of gastrointestinal damage and inflammatory cytokines. Blood 90, 3204-3213.

Hill, G.R., Teshima, T., Gerbitz, a, Pan, L., Cooke, K.R., Brinson, Y.S., Crawford, J.M., and Ferrara, J.L. (1999). Differential roles of IL-1 and TNF-alpha on graft-versus-host disease and graft versus leukemia. J. Clin. Invest. 104, 459-467.

Ho, V.T., Vanneman, M., Kim, H., Sasada, T., Kang, Y.J., Pasek, M., Cutler, C., Koreth, J., Alyea, E., Sarantopoulos, S., et al. (2009). Biologic activity of irradiated, autologous, GM-CSF-secreting leukemia cell vaccines early after allogeneic stem cell transplantation. Proc. Natl. Acad. Sci. U. S. 
Hou, Y., Lin, H., Zhu, L., Liu, Z., Hu, F., Shi, J., Yang, T., Shi, X., Zhu, M., Godley, B.F., et al. (2013). Lipopolysaccharide Increases the Incidence of Collagen-Induced Arthritis in Mice Through Induction of Protease HTRA-1 Expression. ARTHRITIS Rheum. 65, 2835-2846.

Hülsdünker, J., and Zeiser, R. (2015). Insights into the pathogenesis of GvHD: what mice can teach us about man. Tissue Antigens 85, 2-9.

Ichiba, T., Teshima, T., Kuick, R., Misek, D.E., Liu, C., Takada, Y., Maeda, Y., Reddy, P., Williams, D.L., Hanash, S.M., et al. (2003). Early changes in gene expression profiles of hepatic GVHD uncovered by oligonucleotide microarrays. Blood 102, 763-771.

Iclozan, C., Yu, Y., Liu, C., Liang, Y., Yi, T., Anasetti, C., and Yu, X.-Z. (2010). T helper17 cells are sufficient but not necessary to induce acute graft-versus-host disease. Biol. Blood Marrow Transplant. 16, 170-178.

Isernhagen, A., Malzahn, D., Viktorova, E., Elsner, L., Monecke, S., von Bonin, F., Kilisch, M., Wermuth, J.M., Walther, N., Balavarca, Y., et al. (2015). The MICA-129 dimorphism affects NKG2D signaling and outcome of hematopoietic stem cell transplantation. EMBO Mol. Med. 7, $1480-1502$.

Isernhagen, A., Schilling, D., Monecke, S., Shah, P., Elsner, L., Walter, L., Multhoff, G., and Dressel, R. (2016a). The MICA-129Met/Val dimorphism affects plasma membrane expression and shedding of the NKG2D ligand MICA. Immunogenetics 68, 109-123.

Isernhagen, A., Malzahn, D., Monecke, S., Schilling, D., Shah, P., Multhoff, G., Wulf, G., Kube, D., Bickeböller, H., and Dressel, R. (2016b). Functional consequences of genetic polymorphisms in the NKG2D receptor signaling pathway and putative gene interactions. Recept. Clin. Investig. 3.

Jaksch, M., and Mattsson, J. (2005). The pathophysiology of acute graft-versus-host disease. Scand. J. Immunol. 61, 398-409.

Jaksch, M., Remberger, M., and Mattsson, J. (2005). Increased gene expression of chemokine receptors is correlated with acute graft-versus-host disease after allogeneic stem cell transplantation. Biol. Blood Marrow Transplant. 11, 280-287.

Jasperson, L.K., Bucher, C., Panoskaltsis-Mortari, A., Mellor, A.L., Munn, D.H., and Blazar, B.R. (2009). Inducing the tryptophan catabolic pathway, indoleamine 2,3-dioxygenase (IDO), for suppression of graft-versus-host disease (GVHD) lethality. Blood 114, 5062-5070.

Karimi, M.A., Bryson, J.L., Richman, L.P., Fesnak, A.D., Leichner, T.M., Satake, A., Vonderheide, R.H., Raulet, D.H., Reshef, R., and Kambayashi, T. (2015a). NKG2D expression by CD8+ T cells contributes to GVHD and GVT effects in a murine model of allogeneic HSCT. Blood 125, 3655-3663.

Karimi, M.A., Bryson, J.L., Richman, L.P., Fesnak, A.D., Leichner, T.M., Satake, A., Vonderheide, R.H., Raulet, D.H., Reshef, R., and Kambayashi, T. (2015b). NKG2D expression by CD8+ T cells contributes to GVHD and GVT effects in a murine model of allogeneic HSCT. Blood 125, 3655-3663.

Kichian, K., Nestel, F.P., Kim, D., Ponka, P., and Lapp, W.S. (1996). IL-12 p40 messenger RNA expression in target organs during acute graft-versus-host disease. Possible involvement of IFNgamma. J. Immunol. 157, 2851-2856. 
Kitko, C.L., Levine, J.E., Storer, B.E., Chai, X., Fox, D.A., Braun, T.M., Couriel, D.R., Martin, P.J., Flowers, M.E., Hansen, J.A., et al. (2014). Plasma CXCL9 elevations correlate with chronic GVHD diagnosis. Blood 123, 786-793.

Kong, N., Lan, Q., Chen, M., Wang, J., Shi, W., Horwitz, D.A., Quesniaux, V., Ryffel, B., Liu, Z., Brand, D., et al. (2012). Antigen-specific transforming growth factor $\beta$-induced Treg cells, but not natural Treg cells, ameliorate autoimmune arthritis in mice by shifting the Th17/Treg cell balance from Th17 predominance to Treg cell predominance. Arthritis Rheum. 64, 2548-2558.

Koreth, J., Matsuoka, K., Kim, H.T., McDonough, S.M., Bindra, B., Alyea, E.P., Armand, P., Cutler, C., Ho, V.T., Treister, N.S., et al. (2011). Interleukin-2 and regulatory T cells in graftversus-host disease. N. Engl. J. Med. 365, 2055-2066.

Korn, T., Bettelli, E., Oukka, M., and Kuchroo, V.K. (2009). IL-17 and Th17 Cells. Annu. Rev. Immunol. 27, 485-517.

Korngold, R., and Sprent, J. (1980). Negative selection of T cells causing lethal graft-versus-host disease across minor histocompatibility barriers. Role of the H-2 complex. J. Exp. Med. 151, 11141124.

Korngold, R., and Sprent, J. (1982). Features of T cells causing H-2-restricted lethal graft-vs.-host disease across minor histocompatibility barriers. J. Exp. Med. 155, 872-883.

Korngold, R., and Sprent, J. (1985). Surface markers of T cells causing lethal graft-vs-host disease to class I vs class II H-2 differences. J. Immunol. 135, 3004-3010.

Kreisel, D., Krupnick, A.S., Balsara, K.R., Riha, M., Gelman, A.E., Popma, S.H., Szeto, W.Y., Turka, L. a, and Rosengard, B.R. (2002). Mouse vascular endothelium activates CD8+ T lymphocytes in a B7-dependent fashion. J. Immunol. 169, 6154-6161.

Krenger, W., Falzarano, G., Delmonte, J., Snyder, K.M., Byon, J.C., and Ferrara, J.L. (1996). Interferon-gamma suppresses $\mathrm{T}$-cell proliferation to mitogen via the nitric oxide pathway during experimental acute graft-versus-host disease. Blood 88, 1113-1121.

Kriegeskorte, A.K., Gebhardt, F.E., Porcellini, S., Schiemann, M., Stemberger, C., Franz, T.J., Huster, K.M., Carayannopoulos, L.N., Yokoyama, W.M., Colonna, M., et al. (2005). NKG2Dindependent suppression of T cell proliferation by H60 and MICA. Proc. Natl. Acad. Sci. U. S. A. $102,11805-11810$.

Kurt-Jones, E.A., Hamberg, S., Ohara, J., Paul, W.E., and Abbas, A.K. (1987). Heterogeneity of helper/inducer T lymphocytes. I. Lymphokine production and lymphokine responsiveness. J. Exp. Med. 166, 1774-1787.

Kurtzberg, J., Laughlin, M., Graham, M.L., Smith, C., Olson, J.F., Halperin, E.C., Ciocci, G., Carrier, C., Stevens, C.E., and Rubinstein, P. (1996). Placental blood as a source of hematopoietic stem cells for transplantation into unrelated recipients. N. Engl. J. Med. 335, 157-166.

Langrehr, J.M., Murase, N., Markus, P.M., Cai, X., Neuhaus, P., Schraut, W., Simmons, R.L., and Hoffman, R.A. (1992). Nitric oxide production in host-versus-graft and graft-versus-host reactions in the rat. J. Clin. Invest. 90, 679-683.

Lechler, R., Ng, W.F., Steinman, R.M., Adler, A.J., Marsh, D.W., Yochum, G.S., Guzzo, J.L., Nigam, A., Nelson, W.G., Pardoll, D.M., et al. (2001). Dendritic Cells in Transplantation-Friend or Foe? Immunity 14, 357-368. 
Lee, S.J., Klein, J., Haagenson, M., Baxter-Lowe, L.A., Confer, D.L., Eapen, M., Fernandez-Vina, M., Flomenberg, N., Horowitz, M., Hurley, C.K., et al. (2007). High-resolution donor-recipient HLA matching contributes to the success of unrelated donor marrow transplantation. Blood 110, 4576-4583.

Li, J., Nishizawa, K., An, W., Hussey, R.E., Lialios, F.E., Salgia, R., Sunder-Plassmann, R., and Reinherz, E.L. (1998). A cdc15-like adaptor protein (CD2BP1) interacts with the CD2 cytoplasmic domain and regulates CD2-triggered adhesion. EMBO J. 17, 7320-7336.

Li, Q., Zhai, Z., Xu, X., Shen, Y., Zhang, A., Sun, Z., Liu, H., Geng, L., and Wang, Y. (2010). Decrease of $\mathrm{CD} 4(+) \mathrm{CD} 25(+)$ regulatory T cells and TGF-beta at early immune reconstitution is associated to the onset and severity of graft-versus-host disease following allogeneic haematogenesis stem cell transplantation. Leuk. Res. 34, 1158-1168.

Liu, R., Paxton, W.A., Choe, S., Ceradini, D., Martin, S.R., Horuk, R., MacDonald, M.E., Stuhlmann, H., Koup, R.A., and Landau, N.R. (1996). Homozygous defect in HIV-1 coreceptor accounts for resistance of some multiply-exposed individuals to HIV-1 infection. Cell 86, 367-377.

Loiseau, P., Busson, M., Balere, M.-L., Dormoy, A., Bignon, J.-D., Gagne, K., Gebuhrer, L., Dubois, V., Jollet, I., Bois, M., et al. (2007). HLA Association with hematopoietic stem cell transplantation outcome: the number of mismatches at HLA-A, -B, -C, -DRB1, or -DQB1 is strongly associated with overall survival. Biol. Blood Marrow Transplant. 13, 965-974.

Lord, J.D., Hackman, R.C., Gooley, T.A., Wood, B.L., Moklebust, A.C., Hockenbery, D.M., Steinbach, G., Ziegler, S.F., and McDonald, G.B. (2011). Blood and Gastric FOXP3+ T Cells Are Not Decreased in Human Gastric Graft-versus-Host Disease. Biol. Blood Marrow Transplant. 17, 486-496.

Lu, N.Z., Collins, J.B., Grissom, S.F., and Cidlowski, J.A. (2007). Selective regulation of bone cell apoptosis by translational isoforms of the glucocorticoid receptor. Mol. Cell. Biol. 27, 7143-7160.

Ma, H.-H.H., Ziegler, J., Li, C., Sepulveda, A., Bedeir, A., Grandis, J., Lentzsch, S., and Mapara, M.Y. (2011). Sequential activation of inflammatory signaling pathways during graft-versus-host disease (GVHD): early role for STAT1 and STAT3. Cell. Immunol. 268, 37-46.

Mabed, M., Maroof, S., Zalta, K., and El-Awadee, M. (2005). Delayed or delayed sequential bone marrow transplantation: relevance for acute graft-versus-host disease prevention after major $\mathrm{H} 2$ incompatible transplantation. Bone Marrow Transplant. 35, 803-806.

Malarkannan, S., Shih, P.P., Eden, P.A., Horng, T., Zuberi, A.R., Christianson, G., Roopenian, D., and Shastri, N. (1998). The molecular and functional characterization of a dominant minor $\mathrm{H}$ antigen, H60. J. Immunol. 161, 3501-3509.

Manickasingham, S.P., Anderton, S.M., Burkhart, C., and Wraith, D.C. (1998). Qualitative and quantitative effects of CD28/B7-mediated costimulation on naive T cells in vitro. J. Immunol. 161, $3827-3835$.

Mapara, M.Y., Leng, C., Kim, Y.-M., Bronson, R., Lokshin, A., Luster, A., and Sykes, M. (2006). Expression of chemokines in GVHD target organs is influenced by conditioning and genetic factors and amplified by GVHR. Biol. Blood Marrow Transplant. 12, 623-634.

Matzinger, P. (2002). The danger model: a renewed sense of self. Science 296, 301-305.

Mauri, C., and Ehrenstein, M.R. (2008). The "short" history of regulatory B cells. Trends Immunol. 29, 34-40. 
Mestas, J., and Hughes, C.C. (2001). Endothelial cell costimulation of T cell activation through CD58-CD2 interactions involves lipid raft aggregation. J. Immunol. 167, 4378-4385.

Mestas, J., and Hughes, C.C.W. (2004). Of mice and not men: differences between mouse and human immunology. J. Immunol. 172, 2731-2738.

Miura, Y., Thoburn, C.J., Bright, E.C., Phelps, M.L., Shin, T., Matsui, E.C., Matsui, W.H., Arai, S., Fuchs, E.J., Vogelsang, G.B., et al. (2004). Association of Foxp3 regulatory gene expression with graft-versus-host disease. Blood 104, 2187-2193.

Mohty, M., Blaise, D., Faucher, C., Vey, N., Bouabdallah, R., Stoppa, A.M., Viret, F., Gravis, G., Olive, D., and Gaugler, B. (2005). Inflammatory cytokines and acute graft-versus-host disease after reduced-intensity conditioning allogeneic stem cell transplantation. Blood 106, 4407-4411.

Moll, N.M., and Ransohoff, R.M. (2010). CXCL12 and CXCR4 in bone marrow physiology. Expert Rev. Hematol. 3, 315-322.

Moser, B., Wolf, M., Walz, A., and Loetscher, P. (2004). Chemokines: multiple levels of leukocyte migration control. Trends Immunol. 25, 75-84.

Mulcahy, H., O'Rourke, K.P., Adams, C., Molloy, M.G., and O'Gara, F. (2006). LST1 and NCR3 expression in autoimmune inflammation and in response to IFN- $\gamma$, LPS and microbial infection. Immunogenetics 57, 893-903.

Müller-Ruchholtz, W., Wottge, H.U., and Müller-Hermelink, H.K. (1976). Bone marrow transplantation in rats across strong histocompatibility barriers by selective elimination of lymphoid cells in donor marrow. Transplant. Proc. 8, 537-541.

Murai, M., Yoneyama, H., Harada, A., Yi, Z., Vestergaard, C., Guo, B., Suzuki, K., Asakura, H., and Matsushima, K. (1999). Active participation of CCR5+CD8+ T lymphocytes in the pathogenesis of liver injury in graft-versus-host disease. J. Clin. Invest. 104, 49-57.

Murphy, L.L., Mazanet, M.M., Taylor, A.C., Mestas, J., and Hughes, C.C. (1999). Single-cell analysis of costimulation by B cells, endothelial cells, and fibroblasts demonstrates heterogeneity in responses of CD4(+) memory T cells. Cell. Immunol. 194, 150-161.

Nabekura, T., Shibuya, K., Takenaka, E., Kai, H., Shibata, K., Yamashita, Y., Harada, K., TaharaHanaoka, S., Honda, S., and Shibuya, A. (2010). Critical role of DNAX accessory molecule-1 (DNAM-1) in the development of acute graft-versus-host disease in mice. Proc. Natl. Acad. Sci. U. S. A. 107, 18593-18598.

Nagata, S., and Golstein, P. (1995). The Fas death factor. Science 267, 1449-1456.

Napolitani, G., Acosta-Rodriguez, E. V, Lanzavecchia, A., and Sallusto, F. (2009). Prostaglandin E2 enhances Th17 responses via modulation of IL-17 and IFN-gamma production by memory CD4+ T cells. Eur. J. Immunol. 39, 1301-1312.

Nestel, F.P., Price, K.S., Seemayer, T.A., and Lapp, W.S. (1992). Macrophage priming and lipopolysaccharide-triggered release of tumor necrosis factor alpha during graft-versus-host disease. J. Exp. Med. 175, 405-413.

New, J.Y., Li, B., Koh, W.P., Ng, H.K., Tan, S.Y., Yap, E.H., Chan, S.H., and Hu, H.Z. (2002). T cell infiltration and chemokine expression: relevance to the disease localization in murine graftversus-host disease. Bone Marrow Transplant. 29, 979-986.

Ni, M., MacFarlane, A.W., Toft, M., Lowell, C.A., Campbell, K.S., and Hamerman, J.A. (2012). 
B-cell adaptor for PI3K (BCAP) negatively regulates Toll-like receptor signaling through activation of PI3K. Proc. Natl. Acad. Sci. U. S. A. 109, 267-272.

Novota, P., Sviland, L., Zinocker, S., Stocki, P., Balavarca, Y., Bickeboller, H., Rolstad, B., Wang, X.N., Dickinson, A.M., and Dressel, R. (2008). Correlation of Hsp70-1 and Hsp70-2 gene expression with the degree of graft-versus-host reaction in a rat skin explant model. Transplantation 85, 1809-1816.

Novota, P., Zinocker, S., Norden, J., Wang, X.N., Sviland, L., Opitz, L., Salinas-Riester, G., Rolstad, B., Dickinson, A.M., Walter, L., et al. (2011). Expression profiling of major histocompatibility and natural killer complex genes reveals candidates for controlling risk of graft versus host disease. PLoS One 6, e16582.

Ocklenburg, F., Moharregh-Khiabani, D., Geffers, R., Janke, V., Pfoertner, S., Garritsen, H., Groebe, L., Klempnauer, J., Dittmar, K.E.J., Weiss, S., et al. (2006). UBD, a downstream element of FOXP3, allows the identification of LGALS3, a new marker of human regulatory T cells. Lab. Invest. 86, 724-737.

Ogasawara, K., Hamerman, J.A., Ehrlich, L.R., Bour-Jordan, H., Santamaria, P., Bluestone, J.A., and Lanier, L.L. (2004). NKG2D blockade prevents autoimmune diabetes in NOD mice. Immunity $20,757-767$.

OKunewick, J.P., Buffo, M.J., and Kociban, D.L. (1987). Evidence for two distinct mechanisms in acute fatal graft-versus-host reaction across minor histocompatibility barriers. Exp. Hematol. 15, $365-372$.

Olson, J. a., Leveson-Gower, D.B., Gill, S., Baker, J., Beilhack, A., and Negrin, R.S. (2010). NK cells mediate reduction of GVHD by inhibiting activated, alloreactive T cells while retaining GVT effects. Blood 115, 4293-4301.

Ordemann, R., Hutchinson, R., Friedman, J., Burakoff, S.J., Reddy, P., Duffner, U., Braun, T.M., Liu, C., Teshima, T., and Ferrara, J.L.M. (2002). Enhanced allostimulatory activity of host antigenpresenting cells in old mice intensifies acute graft-versus-host disease. J. Clin. Invest. 109, 12491256.

Pan, W., da Graca, L.S., Shao, Y., Yin, Q., Wu, H., and Jiang, X. (2009). PHAPI/pp32 suppresses tumorigenesis by stimulating apoptosis. J. Biol. Chem. 284, 6946-6954.

Park, H., Li, Z., Yang, X.O., Chang, S.H., Nurieva, R., Wang, Y.-H., Wang, Y., Hood, L., Zhu, Z., Tian, Q., et al. (2005). A distinct lineage of CD4 T cells regulates tissue inflammation by producing interleukin 17. Nat. Immunol. 6, 1133-1141.

Pasquini MC, Wang Z, S.L. (2007). Current use and outcome of hematopoietic stem cell transplantation: part I-CIBMTR Summary Slides, 2007. CIBMTR Newsl. 13, 5-9.

Petersdorf, E.W. (2007). Risk assessment in haematopoietic stem cell transplantation: histocompatibility. Best Pr. Res Clin Haematol 20, 155-170.

Pfoertner, S., Jeron, A., Probst-Kepper, M., Guzman, C.A., Hansen, W., Westendorf, A.M., Toepfer, T., Schrader, A.J., Franzke, A., Buer, J., et al. (2006). Signatures of human regulatory T cells: an encounter with old friends and new players. Genome Biol. 7, R54.

Pober JS, Kluger MS, S.J. (2001). Human endothelial cell presentation of antigen and the homing of memory/effector T cells to skin. Ann N Y Acad Sci 941, 12-25. 
Poloni, A., Sartini, D., Emanuelli, M., Trappolini, S., Mancini, S., Pozzi, V., Costantini, B., Serrani, F., Berardinelli, E., Renzi, E., et al. (2011). Gene expression profile of cytokines in patients with chronic graft-versus-host disease after allogeneic hematopoietic stem cell transplantation with reduced conditioning. Cytokine 53, 376-383.

Pomerantz, J.L., Denny, E.M., and Baltimore, D. (2002). CARD11 mediates factor-specific activation of NF-kappaB by the T cell receptor complex. EMBO J. 21, 5184-5194.

Ratajczak, P., Janin, A., Peffault de Latour, R., Leboeuf, C., Desveaux, A., Keyvanfar, K., Robin, M., Clave, E., Douay, C., Quinquenel, A., et al. (2010). Th17/Treg ratio in human graft-versus-host disease. Blood 116, 1165-1171.

Raulet, D.H. (2003). Roles of the NKG2D immunoreceptor and its ligands. Nat. Rev. Immunol. 3, 781-790.

Raulet, D.H., Gasser, S., Gowen, B.G., Deng, W., and Jung, H. (2013). Regulation of ligands for the NKG2D activating receptor. Annu. Rev. Immunol. 31, 413-441.

Ray, A., and Prefontaine, K.E. (1994). Physical association and functional antagonism between the p65 subunit of transcription factor NF-kappa B and the glucocorticoid receptor. Proc. Natl. Acad. Sci. U. S. A. $91,752-756$.

Rébé, C., Raveneau, M., Chevriaux, A., Lakomy, D., Sberna, A.-L., Costa, A., Bessède, G., Athias, A., Steinmetz, E., Lobaccaro, J.M.A., et al. (2009). Induction of transglutaminase 2 by a liver X receptor/retinoic acid receptor alpha pathway increases the clearance of apoptotic cells by human macrophages. Circ. Res. 105, 393-401.

Reddy, P. (2003). Pathophysiology of acute graft-versus-host disease. Hematol. Oncol. 21, 149161.

Reddy, P., and Ferrara, J.L.. M. (2003). Immunobiology of acute graft-versus-host disease. Blood Rev. 17, 187-194.

Reichenbach, D.K., Schwarze, V., Matta, B.M., Tkachev, V., Lieberknecht, E., Liu, Q., Koehn, B.H., Pfeifer, D., Taylor, P.A., Prinz, G., et al. (2015). The IL-33/ST2 axis augments effector Tcell responses during acute GVHD. Blood 125, 3183-3192.

Remberger, M., Ringdén, O., Blau, I.W., Ottinger, H., Kremens, B., Kiehl, M.G., Aschan, J., Beelen, D.W., Basara, N., Kumlien, G., et al. (2001). No difference in graft-versus-host disease, relapse, and survival comparing peripheral stem cells to bone marrow using unrelated donors. Blood 98, 1739-1745.

Ren, G., Zhao, X., Zhang, L., Zhang, J., L’Huillier, A., Ling, W., Roberts, A.I., Le, A.D., Shi, S., Shao, C., et al. (2010). Inflammatory cytokine-induced intercellular adhesion molecule-1 and vascular cell adhesion molecule-1 in mesenchymal stem cells are critical for immunosuppression. J. Immunol. 184, 2321-2328.

Rezvani, K., Mielke, S., Ahmadzadeh, M., Kilical, Y., Savani, B.N., Zeilah, J., Keyvanfar, K., Montero, A., Hensel, N., Kurlander, R., et al. (2006). High donor FOXP3-positive regulatory T-cell (Treg) content is associated with a low risk of GVHD following HLA-matched allogeneic SCT. Blood 108, 1291-1297.

Ringdén, O. (1992). Correlation of pretransplant viral serology and complications of bone marrow transplantation. Ann. Hematol. 64 Suppl, A143-A147. 
Ringdén, O., Remberger, M., Runde, V., Bornhäuser, M., Blau, I.W., Basara, N., Hölig, K., Beelen, D.W., Hägglund, H., Basu, O., et al. (2000). Faster engraftment of neutrophils and platelets with peripheral blood stem cells from unrelated donors: a comparison with marrow transplantation. Bone Marrow Transplant. 25 Suppl 2, S6-S8.

Ringdén, O., Labopin, M., Bacigalupo, A., Arcese, W., Schaefer, U.W., Willemze, R., Koc, H., Bunjes, D., Gluckman, E., Rocha, V., et al. (2002). Transplantation of peripheral blood stem cells as compared with bone marrow from HLA-identical siblings in adult patients with acute myeloid leukemia and acute lymphoblastic leukemia. J. Clin. Oncol. 20, 4655-4664.

Rodriguez, P.C., Quiceno, D.G., Zabaleta, J., Ortiz, B., Zea, A.H., Piazuelo, M.B., Delgado, A., Correa, P., Brayer, J., Sotomayor, E.M., et al. (2004). Arginase I production in the tumor microenvironment by mature myeloid cells inhibits T-cell receptor expression and antigen-specific T-cell responses. Cancer Res. 64, 5839-5849.

Ruggeri, L., Capanni, M., Urbani, E., Perruccio, K., Shlomchik, W.D., Tosti, A., Posati, S., Rogaia, D., Frassoni, F., Aversa, F., et al. (2002). Effectiveness of donor natural killer cell alloreactivity in mismatched hematopoietic transplants. Science 295, 2097-2100.

Sackstein, R. (1995). Lymphocyte migration following bone marrow transplantation. Ann. N. Y. Acad. Sci. 770, 177-188.

Sadeghi, B., Al-Chaqmaqchi, H., Al-Hashmi, S., Brodin, D., Hassan, Z., Abedi-Valugerdi, M., Moshfegh, A., and Hassan, M. (2013). Early-phase GVHD gene expression profile in target versus non-target tissues: kidney, a possible target? Bone Marrow Transplant. 48, 284-293.

Sakaguchi, S., Sakaguchi, N., Asano, M., Itoh, M., and Toda, M. (1995). Immunologic selftolerance maintained by activated $\mathrm{T}$ cells expressing IL-2 receptor alpha-chains (CD25). Breakdown of a single mechanism of self-tolerance causes various autoimmune diseases. J. Immunol. 155, 1151-1164.

Sallusto, F., Lanzavecchia, A., and Mackay, C.R. (1998). Chemokines and chemokine receptors in T-cell priming and Th1/Th2-mediated responses. Immunol. Today 19, 568-574.

Sarantopoulos, S., Stevenson, K.E., Kim, H.T., Cutler, C.S., Bhuiya, N.S., Schowalter, M., Ho, V.T., Alyea, E.P., Koreth, J., Blazar, B.R., et al. (2009). Altered B-cell homeostasis and excess BAFF in human chronic graft-versus-host disease. Blood 113, 3865-3874.

Saxena, M., Williams, S., Gilman, J., and Mustelin, T. (1998). Negative regulation of T cell antigen receptor signal transduction by hematopoietic tyrosine phosphatase (HePTP). J. Biol. Chem. 273, $15340-15344$.

Schmaltz, C., Alpdogan, O., Kappel, B.J., Muriglan, S.J., Rotolo, J.A., Ongchin, J., Willis, L.M., Greenberg, A.S., Eng, J.M., Crawford, J.M., et al. (2002). T cells require TRAIL for optimal graftversus-tumor activity. Nat. Med. 8, 1433-1437.

Schmaltz, C., Alpdogan, O., Muriglan, S.J., Kappel, B.J., Rotolo, J.A., Ricchetti, E.T., Greenberg, A.S., Willis, L.M., Murphy, G.F., Crawford, J.M., et al. (2003). Donor T cell-derived TNF is required for graft-versus-host disease and graft-versus-tumor activity after bone marrow transplantation. Blood 101, 2440-2445.

Schroeder, M.A., and DiPersio, J.F. (2011). Mouse models of graft-versus-host disease: advances and limitations. Dis. Model. Mech. 4, 318-333.

Schultz, K.R., Paquet, J., Bader, S., and HayGlass, K.T. (1995). Requirement for B cells in T cell priming to minor histocompatibility antigens and development of graft-versus-host disease. Bone 
Marrow Transplant. 16, 289-295.

Schwarte, S., and Hoffmann, M.W. (2005). Influence of radiation protocols on graft-vs-host disease incidence after bone-marrow transplantation in experimental models. Methods Mol. Med. $109,445-458$.

Seok, J., Warren, H.S., Cuenca, A.G., Mindrinos, M.N., Baker, H. V, Xu, W., Richards, D.R., McDonald-Smith, G.P., Gao, H., Hennessy, L., et al. (2013). Genomic responses in mouse models poorly mimic human inflammatory diseases. Proc. Natl. Acad. Sci. U. S. A. 110, 3507-3512.

Shafi, S., Vantourout, P., Wallace, G., Antoun, A., Vaughan, R., Stanford, M., and Hayday, A. (2011). An NKG2D-mediated human lymphoid stress surveillance response with high interindividual variation. Sci. Transl. Med. 3, 113ra124.

Shibuya, A., Campbell, D., Hannum, C., Yssel, H., Franz-Bacon, K., McClanahan, T., Kitamura, T., Nicholl, J., Sutherland, G.R., Lanier, L.L., et al. (1996). DNAM-1, a novel adhesion molecule involved in the cytolytic function of T lymphocytes. Immunity 4, 573-581.

Shimabukuro-Vornhagen, A., Hallek, M.J., Storb, R.F., and von Bergwelt-Baildon, M.S. (2009). The role of B cells in the pathogenesis of graft-versus-host disease. Blood 114, 4919-4927.

Shlomchik, W.D. (2007). Graft-versus-host disease. Nat. Rev. Immunol. 7, 340-352.

Shlomchik, W.D., Couzens, M.S., Tang, C.B., McNiff, J., Robert, M.E., Liu, J., Shlomchik, M.J., and Emerson, S.G. (1999). Prevention of graft versus host disease by inactivation of host antigenpresenting cells. Science 285, 412-415.

Shlomchik, W.D., Lucarelli, G., Clift, R.A., Galimberti, M., al., et, Lucarelli, G., Galimberti, M., Giardini, C., al., et, Boulad, F., et al. (2003). Antigen presentation in graft-vs-host disease. Exp. Hematol. 31, 1187-1197.

Skert, C., Damiani, D., Michelutti, a, Patriarca, F., Arpinati, M., Filì, C., Lucchi, P., Malagola, M., Bergonzi, C., Roccaro, a, et al. (2009). Kinetics of Th1/Th2 cytokines and lymphocyte subsets to predict chronic GVHD after allo-SCT: results of a prospective study. Bone Marrow Transplant. 44, $729-737$.

Snover, D.C., Weisdorf, S.A., Ramsay, N.K., McGlave, P., and Kersey, J.H. (1984). Hepatic graft versus host disease: a study of the predictive value of liver biopsy in diagnosis. Hepatology 4, $123-$ 130.

Snover, D.C., Weisdorf, S.A., Vercellotti, G.M., Rank, B., Hutton, S., and McGlave, P. (1985). A histopathologic study of gastric and small intestinal graft-versus-host disease following allogeneic bone marrow transplantation. Hum. Pathol. 16, 387-392.

Sprent, J., Schaefer, M., Lo, D., and Korngold, R. (1986). Properties of purified T cell subsets. II. In vivo responses to class I vs. class II H-2 differences. J. Exp. Med. 163, 998-1011.

Sprent, J., Schaefer, M., Gao, E.K., and Korngold, R. (1988). Role of T cell subsets in lethal graftversus-host disease (GVHD) directed to class I versus class II H-2 differences. I. L3T4+ cells can either augment or retard GVHD elicited by Lyt-2+ cells in class I different hosts. J. Exp. Med. 167, $556-569$.

St-Pierre, Y., Campion, C.G., and Grosset, A.-A. (2012). A distinctive role for galectin-7 in cancer? Front. Biosci. (Landmark Ed. 17, 438-450. 
Storek, J., Gooley, T., Siadak, M., Bensinger, W.I., Maloney, D.G., Chauncey, T.R., Flowers, M., Sullivan, K.M., Witherspoon, R.P., Rowley, S.D., et al. (1997). Allogeneic peripheral blood stem cell transplantation may be associated with a high risk of chronic graft-versus-host disease. Blood 90, 4705-4709.

Stuart, J.M., Segal, E., Koller, D., and Kim, S.K. (2003). A gene-coexpression network for global discovery of conserved genetic modules. Science 302, 249-255.

Sugerman, P.B., Faber, S.B., Willis, L.M., Petrovic, A., Murphy, G.F., Pappo, J., Silberstein, D., and van den Brink, M.R.M. (2004). Kinetics of gene expression in murine cutaneous graft-versushost disease. Am. J. Pathol. 164, 2189-2202.

Tahara-Hanaoka, S., Shibuya, K., Onoda, Y., Zhang, H., Yamazaki, S., Miyamoto, A., Honda, S.I., Lanier, L.L., and Shibuya, A. (2004). Functional characterization of DNAM-1 (CD226) interaction with its ligands PVR (CD155) and nectin-2 (PRR-2/CD112). Int. Immunol. 16, 533538.

Takada, A., Yoshida, S., Kajikawa, M., Miyatake, Y., Tomaru, U., Sakai, M., Chiba, H., Maenaka, K., Kohda, D., Fugo, K., et al. (2008). Two novel NKG2D ligands of the mouse H60 family with differential expression patterns and binding affinities to NKG2D. J. Immunol. 180, 1678-1685.

Tanaka, J., Imamura, M., Kasai, M., Sakurada, K., and Miyazaki, T. (1995a). Cytokine gene expression after allogeneic bone marrow transplantation. Leuk. Lymphoma 16, 413-418.

Tanaka, J., Imamura, M., Kasai, M., Zhu, X., Kobayashi, S., Hashino, S., Higa, T., Sakurada, K., and Asaka, M. (1995b). Cytokine receptor gene expression in peripheral blood mononuclear cells during graft-versus-host disease after allogeneic bone marrow transplantation. Leuk. Lymphoma 19, 281-287.

Tawara, I., Koyama, M., Liu, C., Toubai, T., Thomas, D., Evers, R., Chockley, P., Nieves, E., Sun, Y., Lowler, K.P., et al. (2011). Interleukin-6 modulates graft-versus-host responses after experimental allogeneic bone marrow transplantation. Clin. Cancer Res. 17, 77-88.

Taylor, P.A., Panoskaltsis-Mortari, A., Freeman, G.J., Sharpe, A.H., Noelle, R.J., Rudensky, A.Y., Mak, T.W., Serody, J.S., and Blazar, B.R. (2005). Targeting of inducible costimulator (ICOS) expressed on alloreactive T cells down-regulates graft-versus-host disease (GVHD) and facilitates engraftment of allogeneic bone marrow (BM). Blood 105, 3372-3380.

Theiss-Suennemann, J., Jörß, K., Messmann, J.J., Reichardt, S.D., Montes-Cobos, E., Lühder, F., Tuckermann, J.P., AWolff, H., Dressel, R., Gröne, H.-J., et al. (2015). Glucocorticoids attenuate acute graft-versus-host disease by suppressing the cytotoxic capacity of CD8(+) T cells. J. Pathol. $235,646-655$.

THOMAS, E.D., LOCHTE, H.L., CANNON, J.H., SAHLER, O.D., and FERREBEE, J.W. (1959). Supralethal whole body irradiation and isologous marrow transplantation in man. J. Clin. Invest. $38,1709-1716$.

Thomas, E.D., Storb, R., Clift, R.A., Fefer, A., Johnson, L., Neiman, P.E., Lerner, K.G., Glucksberg, H., and Buckner, C.D. (1975). Bone-marrow transplantation (second of two parts). N. Engl. J. Med. 292, 895-902.

Trentin, J.J., and Judd, K.P. (1973). Prevention of acute graft-versus-host (GVH) mortality with spleen-absorbed antithymocyte globulin (ATG). Transplant. Proc. 5, 865-868.

Turnbull, I.R., Gilfillan, S., Cella, M., Aoshi, T., Miller, M., Piccio, L., Hernandez, M., and 
Colonna, M. (2006). Cutting Edge: TREM-2 Attenuates Macrophage Activation. J. Immunol. 177, $3520-3524$.

Tyan, M.L. (1973). Modification of severe graft-versus-host disease with antisera to the (theta) antigen or to whole serum. Transplantation 15, 601-604.

Ueha, S., Murai, M., Yoneyama, H., Kitabatake, M., Imai, T., Shimaoka, T., Yonehara, S., Ishikawa, S., and Matsushima, K. (2007). Intervention of MAdCAM-1 or fractalkine alleviates graft-versus-host reaction associated intestinal injury while preserving graft-versus-tumor effects. J. Leukoc. Biol. 81, 176-185.

Uhrberg, M., Valiante, N.M., Young, N.T., Lanier, L.L., Phillips, J.H., and Parham, P. (2001). The repertoire of killer cell Ig-like receptor and CD94:NKG2A receptors in T cells: clones sharing identical alpha beta TCR rearrangement express highly diverse killer cell Ig-like receptor patterns. J. Immunol. 166, 3923-3932.

Varona, R., Cadenas, V., Lozano, M., Moreno-Ortiz, M.C., Kremer, L., Martínez-A, C., and Márquez, G. (2006). CCR6 regulates the function of alloreactive and regulatory CD4+ T cells during acute graft-versus-host disease. Leuk. Lymphoma 47, 1469-1476.

Verner, J., Kabathova, J., Tomancova, A., Pavlova, S., Tichy, B., Mraz, M., Brychtova, Y., Krejci, M., Zdrahal, Z., Trbusek, M., et al. (2012). Gene expression profiling of acute graft-vs-host disease after hematopoietic stem cell transplantation. Exp. Hematol. 40, 899-905.e5.

Wagner, J.E., Rosenthal, J., Sweetman, R., Shu, X.O., Davies, S.M., Ramsay, N.K., McGlave, P.B., Sender, L., and Cairo, M.S. (1996). Successful transplantation of HLA-matched and HLAmismatched umbilical cord blood from unrelated donors: analysis of engraftment and acute graftversus-host disease. Blood 88, 795-802.

Wall, D.A., and Sheehan, K.C. (1994). The role of tumor necrosis factor and interferon gamma in graft-versus-host disease and related immunodeficiency. Transplantation 57, 273-279.

Ware, C.F., VanArsdale, T.L., Crowe, P.D., and Browning, J.L. (1995). The ligands and receptors of the lymphotoxin system. Curr. Top. Microbiol. Immunol. 198, 175-218.

Weaver, C.T., Hatton, R.D., Mangan, P.R., and Harrington, L.E. (2007). IL-17 family cytokines and the expanding diversity of effector T cell lineages. Annu. Rev. Immunol. 25, 821-852.

Weiss, G., Schwaighofer, H., Herold, M., Nachbaur, D., Wachter, H., Niederwieser, D., and Werner, E.R. (1995). Nitric oxide formation as predictive parameter for acute graft-versus-host disease after human allogeneic bone marrow transplantation. Transplantation 60, 1239-1244.

Welniak, L.A., Blazar, B.R., and Murphy, W.J. (2007). Immunobiology of allogeneic hematopoietic stem cell transplantation. Annu. Rev. Immunol. 25, 139-170.

Wolf, D., Wolf, A.M., Fong, D., Rumpold, H., Strasak, A., Clausen, J., and Nachbaur, D. (2007). Regulatory T-cells in the graft and the risk of acute graft-versus-host disease after allogeneic stem cell transplantation. Transplantation 83, 1107-1113.

Wysocki, C. a, Burkett, S.B., Panoskaltsis-Mortari, A., Kirby, S.L., Luster, A.D., McKinnon, K., Blazar, B.R., and Serody, J.S. (2004). Differential roles for CCR5 expression on donor T cells during graft-versus-host disease based on pretransplant conditioning. J. Immunol. 173, 845-854.

Wysocki, C.A., Panoskaltsis-Mortari, A., Blazar, B.R., and Serody, J.S. (2005a). Leukocyte migration and graft-versus-host disease. Blood 105, 4191-4199. 
Wysocki, C.A., Jiang, Q., Panoskaltsis-Mortari, A., Taylor, P.A., McKinnon, K.P., Su, L., Blazar, B.R., and Serody, J.S. (2005b). Critical role for CCR5 in the function of donor CD4+CD25 + regulatory T cells during acute graft-versus-host disease. Blood 106, 3300-3307.

Yagami, A., Orihara, K., Morita, H., Futamura, K., Hashimoto, N., Matsumoto, K., Saito, H., and Matsuda, A. (2010). IL-33 mediates inflammatory responses in human lung tissue cells. J. Immunol. 185, 5743-5750.

Yamazaki, T., and Kurosaki, T. (2003). Contribution of BCAP to maintenance of mature B cells through c-Rel. Nat. Immunol. 4, 780-786.

Yang, Y.G., Sergio, J.J., Pearson, D.A., Szot, G.L., Shimizu, A., and Sykes, M. (1997). Interleukin12 preserves the graft-versus-leukemia effect of allogeneic CD8 T cells while inhibiting CD4dependent graft-versus-host disease in mice. Blood 90, 4651-4660.

Yi, T., Chen, Y., Wang, L., Du, G., Huang, D., Zhao, D., Johnston, H., Young, J., Todorov, I., Umetsu, D.T., et al. (2009). Reciprocal differentiation and tissue-specific pathogenesis of Th1, Th2, and Th17 cells in graft-versus-host disease. Blood 114, 3101-3112.

Zeiser, R., Beilhack, A., and Negrin, R.S. (2006). Acute graft-versus-host disease-challenge for a broader application of allogeneic hematopoietic cell transplantation. Curr. Stem Cell Res. Ther. 1, 203-212.

Zhang, N., Schröppel, B., Lal, G., Jakubzick, C., Mao, X., Chen, D., Yin, N., Jessberger, R., Ochando, J.C., Ding, Y., et al. (2009). Regulatory T Cells Sequentially Migrate from Inflamed Tissues to Draining Lymph Nodes to Suppress the Alloimmune Response. Immunity 30, 458-469. Zhao, B., and Ivashkiv, L.B. (2011). Negative regulation of osteoclastogenesis and bone resorption by cytokines and transcriptional repressors. Arthritis Res. Ther. 13, 234.

Zhuo, M., Fujiki, M., Wang, M., Piard-Ruster, K., Wai, L.-E., Wei, L., Martinez, O.M., and Krams, S.M. (2010). Identification of the rat NKG2D ligands, RAE1L and RRLT, and their role in allograft rejection. Eur. J. Immunol. 40, 1748-1757.

Zingoni, A., Ardolino, M., Santoni, A., and Cerboni, C. (2012). NKG2D and DNAM-1 activating receptors and their ligands in NK-T cell interactions: role in the NK cell-mediated negative regulation of T cell responses. Front. Immunol. 3, 408.

Zinöcker, S., Sviland, L., Dressel, R., and Rolstad, B. (2011). Kinetics of lymphocyte reconstitution after allogeneic bone marrow transplantation: markers of graft-versus-host disease. J. Leukoc. Biol. 90, 177-187. 


\section{Supplement}

\subsection{Expression of genes in aGvHD mice compared to healthy controls in different tissues}

All fold changes for genes regulated in mice with aGvHD compared to healthy controls have been listed in Table 7.1 for all the tissues. Fold changes $>1$ represent upregulation and $<1$ represent downregulation of the genes. The gene-tissue combinations for which the regulation was significant $(\mathrm{p}<0.05)$ are in bold and in red. All genes are listed alphabetically.

Table 7.1 Fold changes of regulated genes in aGvHD mice compared to healthy controls in the different tissues

\begin{tabular}{|c|c|c|c|c|c|c|}
\hline Genes & Liver & Lung & Skin & Spleen & $\begin{array}{c}\text { Small } \\
\text { intestine }\end{array}$ & $\begin{array}{c}\text { Large } \\
\text { intestine }\end{array}$ \\
\hline Anp32a & 1.17 & 0.29 & 0.58 & 6.25 & 5.68 & 0.75 \\
\hline Arg1 & 9.5 & 22.69 & 2.09 & 1.02 & 7.29 & 59.31 \\
\hline Bmpr1a & 3.15 & 7.94 & 1.86 & 1.83 & 0.99 & 1.67 \\
\hline Clqtnf7 & 0.02 & 0.01 & 2.7 & 0.01 & 0 & $0.3 \times 10^{-3}$ \\
\hline Card11 & 0.06 & 0.05 & 0.85 & $0.2 \times 10^{-3}$ & 0.23 & 0.31 \\
\hline Ccl4 & 2.8 & 8.02 & $8.5 \times 10^{7}$ & 2.06 & 16.93 & 11.87 \\
\hline Ccl5 & 0.65 & 0.9 & 1.06 & 0.15 & 0.96 & 0.37 \\
\hline Ccl9 & 1.51 & 31.98 & 29.18 & 5.97 & 3.78 & 11.61 \\
\hline Ccr1 & 17.84 & 6.62 & 47.37 & 4.64 & 4.5 & 6.62 \\
\hline Ccr4 & 1.57 & 0.35 & 0.31 & 0.05 & 0.41 & 1.57 \\
\hline Ccr5 & 3.01 & 5.07 & 0.52 & 1.56 & 3.82 & 3.15 \\
\hline Cd226 & 1 & 4.25 & 10.54 & 0.97 & 10.66 & 4.89 \\
\hline Cx3cl1 & 0.16 & 0.47 & 1.65 & 0.26 & 1.36 & 0.36 \\
\hline Cx3cr1 & 0.24 & 0.97 & 0.31 & 13.34 & 49.71 & 265.77 \\
\hline Cxcl10 & 2.22 & 47.87 & 21.77 & 0.97 & 10.46 & 21.32 \\
\hline Cxcl11 & 0.11 & 65.72 & 15.71 & 2.99 & 133.73 & 64.82 \\
\hline Cxcl15 & 0.07 & 0.3 & 0.29 & 0.19 & 2.57 & 1.08 \\
\hline Cxcl16 & 2.97 & 5.2 & 4.52 & 0.55 & 2.17 & 5.66 \\
\hline Cxcl9 & 10.54 & 9.07 & 20.17 & 0.31 & 3.18 & 10.54 \\
\hline Cxcr3 & 0.41 & 21.16 & $1.9 \times 10^{6}$ & 1.2 & 12.59 & $4.4 \times 10^{10}$ \\
\hline Cxcr4 & 10.11 & 0.27 & 0.42 & 0.51 & 0.07 & 0.25 \\
\hline Enpp1 & 0.07 & 0.17 & 0.76 & 0.71 & 3 & 2 \\
\hline Fcer1g & 2.31 & 3.66 & 6.92 & 0.91 & 1.6 & 2.15 \\
\hline Fcgr3 & 1.04 & 1.24 & 3.19 & 0.79 & 1.65 & 2.24 \\
\hline Foxp3 & 0.15 & 0 & 1062.95 & 0.42 & 3.68 & 37.9 \\
\hline H60a & 3.7 & 0.17 & 2.94 & 0.08 & 0.87 & 3.46 \\
\hline & & & & & & \\
\hline & & & & & & \\
\hline
\end{tabular}




\begin{tabular}{|c|c|c|c|c|c|c|}
\hline Hcls1 & 3.95 & 8.61 & 44.39 & 1.37 & 1.83 & 3.18 \\
\hline Htral & 1.6 & 1.75 & 0.94 & 0.49 & 1.28 & 1.28 \\
\hline Icam1 & 5.66 & 3.54 & 10.13 & 1.5 & 4.13 & 10.55 \\
\hline Ido1 & 0.71 & 36.57 & 3.52 & 1.08 & 93.18 & 30.82 \\
\hline Ifng & 67.99 & 33.76 & $3 \times 10^{6}$ & 5.63 & $1.16 \times 10^{8}$ & 43.6 \\
\hline Illo & 0.03 & 0.08 & $\mathbf{0}$ & 65.51 & 143.75 & 63.27 \\
\hline$I l 12 a$ & 32.25 & 0.62 & $\mathbf{0}$ & 0.11 & 106.86 & 1.84 \\
\hline$I l 13$ & 17.93 & 1.52 & 0.37 & 2.15 & 164.39 & 6.85 \\
\hline$I l 15$ & 0.64 & 0.79 & 1.78 & 0.52 & 0.5 & 0.44 \\
\hline$I l 17 a$ & 208.1 & 2.78 & 3.88 & 17.88 & 786.77 & 14.56 \\
\hline Illr1 & 6.64 & 1.07 & 1.49 & 0.42 & 2.33 & 1.36 \\
\hline Illrl2 & 7.22 & 15.08 & 1.06 & 1.21 & 1.2 & 1.82 \\
\hline$I l 2$ & 1.13 & 5.4 & 34.42 & 0.65 & 239.65 & 3.31 \\
\hline Il22 & 11.56 & $6.4 \times 10^{4}$ & 0.1 & 116.23 & 30.85 & $1.4 \times 10^{2}$ \\
\hline$I l 23 a$ & 0.24 & 0.01 & 0.38 & 11.35 & 5.1 & $4.9 \times 10^{3}$ \\
\hline Il2ra & 38.77 & 7.49 & 1987.75 & 0.69 & 3.53 & 10.21 \\
\hline Il33 & 0.71 & 6.06 & 3.44 & 0.37 & 2.03 & 4.78 \\
\hline Il4 & 133.21 & 0.15 & 59.98 & $2.2 \times 10^{2}$ & 2.84 & 5.48 \\
\hline Il4ra & 1.18 & 19.28 & 4.1 & 0.78 & 3.23 & 4.91 \\
\hline Il5 & 409.74 & 0.29 & 1135.55 & 20.66 & 2378.45 & 684.19 \\
\hline Il6 & 36.49 & 4.57 & 555.19 & 1.95 & 791.68 & $3.2 \times 10^{2}$ \\
\hline Klrk1 & 2.32 & 15.55 & 7.2 & 1.45 & 4.29 & 3.12 \\
\hline Lgals3 & 7.63 & 10.86 & 2.1 & 4.35 & 1.76 & 0.91 \\
\hline Lgals 7 & 19.67 & 0.01 & 0.22 & 2.59 & $6.4 \times 10^{2}$ & 4.56 \\
\hline Lilra5 & 6.84 & 4.01 & 128.6 & 8.58 & $9.8 \times 10^{2}$ & 168.91 \\
\hline Lst1 & 4.04 & 9.63 & 8.38 & 65.59 & 734.91 & 37.68 \\
\hline Msr1 & 1.06 & 5.29 & 106.48 & 9.34 & 9.65 & 13.69 \\
\hline Pik3ap1 & 1.35 & 4.04 & 1.37 & 0.73 & 1.03 & 1.68 \\
\hline Pstpip1 & 9.47 & 1.66 & 1.15 & 1.01 & 2.12 & 4.34 \\
\hline Ptger2 & 1.18 & 0.39 & 0.61 & 0.39 & 1.78 & 1.91 \\
\hline Ptpn7 & 8.14 & 1.19 & 1.38 & 1.43 & 1.81 & 4.09 \\
\hline$P v r$ & 1.85 & 0.52 & 2.56 & 1.06 & 3.38 & 1.41 \\
\hline Pvrl2 & 2.32 & 2.43 & 1.14 & 2.39 & 2.83 & 1.92 \\
\hline Rae1 & 0.05 & 0.02 & 1.4 & 0.55 & 0.09 & 0.1 \\
\hline Tap1 & 0.07 & 1.31 & 0.73 & 0.01 & 0.01 & 1.3 \\
\hline$T g f b 1$ & 1.76 & 5.06 & 2.15 & 0.78 & 1.06 & 2.01 \\
\hline$T g f b r 1$ & 0.08 & 7.88 & 1.24 & 0.41 & 1.03 & 1.41 \\
\hline$T g f b 2$ & 1.02 & 0.8 & 1.31 & 0.24 & 1.03 & 0.64 \\
\hline $\operatorname{Tgm} 2$ & 5.05 & 26.28 & 3.07 & 3.22 & 9.91 & 8.76 \\
\hline$T n f$ & 0.32 & 2.06 & 0.46 & 0.14 & 1.4 & 3.15 \\
\hline Trem2 & 16.48 & 0.1 & 6.06 & 0.07 & $0.1 \times 10^{-4}$ & 1.02 \\
\hline
\end{tabular}




\begin{tabular}{|c|c|c|c|c|c|c|} 
Ubc & 6.12 & 13.97 & 6.04 & 2.04 & 6.74 & 3.29 \\
\hline Ubd & 2.17 & 10.32 & 59.3 & 59.28 & 11.25 & 7.55 \\
\hline Ulbp1 & 4.46 & 4.82 & 0.76 & 1.87 & 1.09 & 1.45 \\
\hline
\end{tabular}

\subsection{Expression of genes in aGvHD mice compared to BM controls in different tissues}

All fold changes for genes regulated in mice with aGvHD compared to BM controls have been listed in Table 7.2 for the different tissues. Fold changes $>1$ represent upregulation and $<1$ represent downregulation of the genes. The gene-tissue combinations for which the regulation was significant $(\mathrm{p}<0.05)$ are in bold and in blue. All genes are listed alphabetically.

Table 7.2 Fold changes of regulated genes in aGvHD mice compared to BM controls in the different tissues

\begin{tabular}{|c|c|c|c|c|c|c|}
\hline Genes & Liver & Lung & Skin & Spleen & $\begin{array}{c}\text { Small } \\
\text { intestine }\end{array}$ & $\begin{array}{c}\text { Large } \\
\text { intestine }\end{array}$ \\
\hline Anp32a & 20.76 & 7.38 & 0.89 & 8.78 & 4.39 & 4.53 \\
\hline Arg1 & 1.48 & 2.04 & 6.16 & 0.45 & 9.92 & 24.65 \\
\hline Bmprla & 1.14 & 0.99 & 2.84 & 1.63 & 0.97 & 1.44 \\
\hline C1qtnf7 & 20.11 & 0.02 & 26.66 & 0.11 & 0.01 & 0.01 \\
\hline Card11 & 0.05 & 0.11 & 3.15 & 0.34 & 0.52 & 0.57 \\
\hline Ccl4 & 3.23 & 4.09 & 25.25 & 0.52 & 11.97 & 14.42 \\
\hline Ccl5 & 2.81 & 3.37 & 8.78 & 0.46 & 2.1 & 6.28 \\
\hline Ccl9 & 0.95 & 3.68 & 4.95 & 1.39 & 3.48 & 9.26 \\
\hline Ccrl & 6.92 & 3.57 & 4.3 & 2.64 & 5.31 & 2.57 \\
\hline Ccr4 & 6.19 & 0.95 & 1.65 & 0.11 & 1.29 & 2.99 \\
\hline Ccr 5 & 1.08 & 3.46 & 0.06 & 0.95 & 7.42 & 3.95 \\
\hline$C d 226$ & 1.44 & 2.67 & 9.16 & 1.61 & 8.52 & 10.8 \\
\hline Cx3cl1 & 0.57 & 0.79 & 1.32 & 0.15 & 0.89 & 1.42 \\
\hline Cx3cr1 & 0.24 & 0.16 & 0.83 & 0.86 & 3.52 & 17.42 \\
\hline Cxcl10 & 0.17 & 2.6 & 3.45 & 0.12 & 7.07 & 3.4 \\
\hline Cxcl11 & 0.1 & 3.29 & 0.68 & 0.13 & 18.33 & 31.09 \\
\hline Cxcl15 & 0.17 & 0.51 & 2.77 & 0.26 & 1.41 & 0.47 \\
\hline Cxcl16 & 1.88 & 0.94 & 2.23 & 0.32 & 2.08 & 3.09 \\
\hline Cxcl9 & 0.22 & 1.16 & 4.19 & 0.53 & 3.02 & 2.41 \\
\hline Cxcr3 & 2.19 & 49.15 & 92.4 & 2.95 & 51.31 & $2.2 \times 10^{4}$ \\
\hline Cxcr4 & 4.21 & 0.67 & 1.22 & 1.12 & 1.24 & 1.87 \\
\hline Enpp1 & 0.11 & 0.43 & 0.41 & 0.58 & 2.6 & 0.78 \\
\hline Fcer1g & 0.92 & 1.35 & 2.04 & 0.37 & 1.76 & 2.24 \\
\hline
\end{tabular}




\begin{tabular}{|c|c|c|c|c|c|c|}
\hline Fcgr 3 & 1.42 & 1.24 & 0.71 & 0.55 & 1.84 & 2.37 \\
\hline Foxp3 & 0 & 0 & 18.91 & 0.3 & 1707.92 & 8.89 \\
\hline H60a & 0.7 & 0.56 & 2.03 & 0.14 & 2.73 & 3.11 \\
\hline Hcls1 & 2.17 & 0.87 & 4.37 & 0.5 & 3.28 & 2.47 \\
\hline Htral & 40.4 & 0.49 & 1.08 & 0.33 & 2.64 & 1.71 \\
\hline Icam1 & 1.92 & 1.07 & 5.09 & 1.02 & 10.5 & 5.38 \\
\hline Ido1 & 2.03 & 1.16 & 5.48 & 0.77 & 12.03 & 2.75 \\
\hline Ifng & 0.77 & 5.41 & 1361.09 & 0.78 & 235.18 & 95.52 \\
\hline Illo & 0.23 & 1.9 & O & 1 & 215.6 & 7334.52 \\
\hline$I l 12 a$ & 5.08 & 0.99 & 0.31 & 1.07 & 92.56 & 5.3 \\
\hline$I l 13$ & 0.99 & 2.14 & 10.76 & 0.82 & 2.52 & 5.22 \\
\hline Ill5 & 0.73 & 0.69 & 1.96 & 0.71 & 0.5 & 0.88 \\
\hline$I l 17 a$ & 161.28 & 0.4 & 0.45 & 5.8 & 1.11 & 0.71 \\
\hline Illr1 & 3.24 & 0.76 & 1.77 & 0.47 & 5.4 & 1.92 \\
\hline Illrl2 & 1.2 & 1.3 & 1.86 & 1.66 & 2.69 & 1.89 \\
\hline$I l 2$ & 0.03 & 1.69 & 0.03 & 0.21 & 0.88 & 1.65 \\
\hline Il22 & 0.04 & 0.64 & 1.44 & 0.01 & 0.49 & 1.4 \\
\hline$I l 23 a$ & 5.85 & 14.58 & 0.27 & 0.14 & 1.19 & $3.8 \times 10^{2}$ \\
\hline Il2ra & 4.07 & 4.33 & 10.12 & 0.4 & 8.77 & 10.08 \\
\hline Il33 & 1.73 & 2.09 & 2.26 & 0.28 & 3.55 & 4.37 \\
\hline Il4 & 0.12 & 0.1 & $3.7 \times 10^{6}$ & 6.6 & 3.13 & 2.76 \\
\hline Il4ra & 1.53 & 0.7 & 1.91 & 0.91 & 2.18 & 1.93 \\
\hline$I 15$ & 0.47 & 2.72 & 1.13 & 4.39 & 7.42 & 1.37 \\
\hline Il6 & 0.64 & 3.25 & 2.9 & 0.36 & 3.37 & 2.66 \\
\hline Klrk1 & 0.78 & 2.14 & 6.42 & 0.69 & 4.64 & 3.67 \\
\hline Lgals3 & 1.57 & 1.17 & 2.53 & 1.32 & 1.26 & 1.63 \\
\hline Lgals7 & 64.07 & 1.96 & 0.16 & 0.38 & 107.56 & 1.24 \\
\hline Lilra5 & 2.37 & 0.54 & 0.14 & 1.06 & 134.34 & 1.2 \\
\hline Lst1 & 1.55 & 1.02 & 5.22 & 1.6 & 5.48 & 3.2 \\
\hline Msr1 & 1.09 & 0.87 & 0.16 & 1.15 & 3.73 & 1.45 \\
\hline Pik3ap1 & 1.42 & 0.63 & 0.62 & 0.36 & 2.02 & 1.78 \\
\hline Pstpip1 & 10.73 & 1.36 & 1.43 & 1.28 & 5.66 & 5.13 \\
\hline Ptger2 & 1.74 & 0.54 & 1.23 & 0.6 & 3.26 & 3.2 \\
\hline Ptpn7 & 10.73 & 0.95 & 3.63 & 1.12 & 5.66 & 5.13 \\
\hline$P v r$ & 2.23 & 1.35 & 8.32 & 1.25 & 4.1 & 5.89 \\
\hline Pvrl2 & 1.3 & 0.63 & 1.48 & 0.51 & 1.82 & 1.21 \\
\hline Rae1 & 0.52 & 0.96 & 2.11 & 4.29 & 20.05 & 1.86 \\
\hline Tap1 & 0.001 & $\overline{0}$ & 0.01 & $\mathbf{0}$ & 0.07 & 0.03 \\
\hline$T g f b 1$ & 1.62 & 0.68 & 1.02 & 0.54 & 1.97 & 2.03 \\
\hline$T g f b r 1$ & 0.1 & 0.65 & 0.89 & 0.56 & 1.19 & 1.23 \\
\hline$T g f b 2$ & 2.78 & 0.8 & 0.97 & 0.58 & 1.61 & 1.97 \\
\hline
\end{tabular}




\begin{tabular}{|c|c|c|c|c|c|c|} 
Tgm2 & 1.59 & 0.85 & 1.25 & 0.64 & 3.44 & 3.94 \\
\hline Tnf & 0.79 & 2.93 & 2.49 & 0.41 & 5.5 & 5.63 \\
\hline Trem2 & 0.04 & 3.07 & 0 & 6.93 & 0.03 & 1.81 \\
\hline Ubc & 0.79 & 1.24 & 4.91 & 1.74 & 3.67 & 3 \\
\hline Ubd & 2.17 & 10.32 & 59.3 & 0.83 & 11.25 & 7.55 \\
\hline Ulbp1 & 2.14 & 0.74 & 1.99 & 1.36 & 2.32 & 1.39 \\
\hline
\end{tabular}

\subsection{Expression of genes in BM mice compared to healthy controls in different tissues}

All fold changes for genes regulated in mice as a result of preconditioning and transplantation in BM control mice compared to healthy controls have been listed in Table 7.3 for the different tissues. Fold changes $>1$ represent upregulation and $<1$ represent downregulation of the genes. The gene-tissue combinations for which the regulation was significant $(\mathrm{p}<0.05)$ are in bold and in green. All genes are listed alphabetically.

Table 7.3 Fold changes of regulated genes due to preconditioning and transplantation procedure in BM control mice compared to healthy controls

\begin{tabular}{|c|c|c|c|c|c|c|}
\hline Genes & Liver & Lung & Skin & Spleen & $\begin{array}{c}\text { Small } \\
\text { intestine }\end{array}$ & $\begin{array}{c}\text { Large } \\
\text { intestine }\end{array}$ \\
\hline Anp32a & 0.06 & 0.04 & 0.65 & 0.71 & 1.29 & 0.17 \\
\hline Arg1 & 6.4 & 11.1 & 0.34 & 2.28 & 0.74 & 2.41 \\
\hline Bmpr1a & 2.76 & 7.99 & 0.66 & 1.12 & 1.02 & 1.16 \\
\hline C1qtnf7 & 0 & 0.52 & 0.1 & 0.04 & 0.05 & 0.04 \\
\hline Card11 & 1.37 & 0.47 & 0.27 & 0.38 & 0.44 & 0.55 \\
\hline Ccl4 & 0.87 & 1.96 & $3.3 \times 10^{5}$ & 3.98 & 1.41 & 0.82 \\
\hline Ccl5 & 0.23 & 0.27 & 0.12 & 0.31 & 0.45 & 0.06 \\
\hline Ccl9 & 1.58 & 8.69 & 5.9 & 4.28 & 1.09 & 1.25 \\
\hline Ccr1 & 2.58 & 1.86 & 11.02 & 1.76 & 0.85 & 2.58 \\
\hline Ccr4 & 0.25 & 0.37 & 0.19 & 0.46 & 0.32 & 0.52 \\
\hline Ccr5 & 2.81 & 1.47 & 8.41 & 1.64 & 0.51 & 0.78 \\
\hline Cd226 & 0.69 & 1.59 & 1.15 & 0.6 & 1.25 & 0.45 \\
\hline Cx3cl1 & 0.28 & 0.59 & 1.26 & 1.69 & 1.53 & 0.26 \\
\hline Cx3cr1 & 1.01 & 6.25 & 0.37 & 15.57 & 14.13 & 15.25 \\
\hline Cxcl10 & 13.08 & 18.44 & 6.31 & 8.03 & 1.48 & 6.28 \\
\hline Cxcl11 & 1.1 & 19.99 & 23.11 & 22.98 & 7.3 & 2.08 \\
\hline Cxcl15 & 0.4 & 0.59 & 0.1 & 0.73 & 1.82 & 2.28 \\
\hline Cxcl16 & 1.58 & 5.55 & 2.02 & 1.72 & 1.04 & 1.83 \\
\hline & & & & & & \\
\hline
\end{tabular}




\begin{tabular}{|c|c|c|c|c|c|c|}
\hline Cxcl9 & 48.67 & 7.83 & 4.82 & 0.59 & 1.05 & 4.37 \\
\hline Cxcr3 & 0.2 & 0.43 & $2.1 \times 10^{4}$ & 0.41 & 0.25 & $1.9 \times 10^{6}$ \\
\hline Cxcr4 & 2.4 & 0.4 & 0.34 & 0.46 & 0.05 & 0.13 \\
\hline Enpp1 & 0.64 & 0.39 & 1.85 & 1.23 & 1.15 & 2.55 \\
\hline Fcer1g & 2.52 & 2.72 & 3.4 & 2.46 & 0.91 & 0.96 \\
\hline Fcgr3 & 0.73 & 1 & 4.48 & 1.42 & 0.9 & 0.94 \\
\hline Foxp3 & 78.95 & 0.6 & 56.22 & 1.39 & $\mathbf{0}$ & 4.26 \\
\hline H60a & 5.26 & 0.3 & 1.45 & 0.58 & 0.32 & 1.11 \\
\hline Hcls1 & 1.82 & 9.9 & 10.16 & 2.75 & 0.56 & 1.29 \\
\hline Htral & 0.4 & 3.56 & 0.86 & 1.5 & 0.49 & 0.75 \\
\hline Icam1 & 2.94 & 3.32 & 1.99 & 1.47 & 0.39 & 1.96 \\
\hline Ido1 & 0.35 & 31.43 & 0.64 & 1.4 & 7.75 & 11.19 \\
\hline Ifng & 88.01 & 6.25 & 2206.47 & 7.23 & $4.9 \times 10^{4}$ & 0.45 \\
\hline Illo & 0.15 & 0.04 & 0.07 & 65.33 & 0.67 & 0.01 \\
\hline$I l 12 a$ & 6.34 & 0.62 & 0.01 & 0.1 & 1.15 & 0.35 \\
\hline$I l 13$ & 18.05 & 0.71 & 0.03 & 2.62 & 65.21 & 1.31 \\
\hline$I l 15$ & 0.88 & 1.14 & 0.91 & 0.74 & 0.99 & 0.51 \\
\hline$I l 17 a$ & 1.29 & 6.88 & 8.61 & 3.08 & 709.71 & 20.37 \\
\hline Illr1 & 2.05 & 1.4 & 0.84 & 0.89 & 0.43 & 0.71 \\
\hline Illrl2 & 6.03 & 11.62 & 0.57 & 0.73 & 0.44 & 0.96 \\
\hline$I l 2$ & 41.47 & 3.19 & 1262.54 & 0.13 & 273.49 & 2.01 \\
\hline Il22 & 293.51 & $1.04 \times 10^{4}$ & 0.07 & $1.04 \times 10^{4}$ & 62.82 & 91.4 \\
\hline$I l 23 a$ & 0.04 & $0.7 \times 10^{-3}$ & 1.42 & 79.06 & 4.28 & 12.84 \\
\hline Il2ra & 9.53 & 1.73 & 196.44 & 1.01 & 0.4 & 1.01 \\
\hline Il33 & 0.41 & 2.9 & 1.52 & 1.35 & 0.57 & 1.09 \\
\hline$I l 4$ & 214.13 & 1.48 & $\mathbf{0}$ & 33.94 & 0.91 & 0.18 \\
\hline Il4ra & 0.77 & 27.39 & 2.15 & 0.87 & 1.49 & 2.54 \\
\hline Il5 & 865.17 & 0.11 & 1001.57 & 4.71 & 320.54 & 498.43 \\
\hline Il6 & 56.92 & 1.41 & 191.63 & 5.43 & 234.62 & $1.2 \times 10^{2}$ \\
\hline Klrk1 & 2.97 & 7.27 & 1.12 & 2.09 & 0.93 & 0.85 \\
\hline Lgals3 & 4.87 & 9.3 & 0.83 & 3.29 & 1.4 & 0.56 \\
\hline Lgals7 & 0.31 & 0.01 & 1.37 & 6.83 & 5.95 & 3.67 \\
\hline Lilra5 & 2.89 & 7.4 & 892.92 & 8.1 & 7.28 & 140.76 \\
\hline Lst 1 & 2.6 & 9.48 & 1.61 & 41.07 & 134.08 & 11.76 \\
\hline Msr1 & 0.98 & 6.09 & 663.75 & 8.13 & 2.58 & 9.47 \\
\hline Pik3ap1 & 0.95 & 6.4 & 2.21 & 2.03 & 0.51 & 0.95 \\
\hline Pstpip1 & 0.88 & 1.22 & 0.8 & 0.8 & 0.37 & 0.85 \\
\hline Ptger2 & 0.68 & 0.71 & 0.5 & 0.66 & 0.55 & 0.61 \\
\hline Ptpn7 & 0.76 & 1.26 & 0.38 & 1.28 & 0.32 & 0.94 \\
\hline$P v r$ & 0.83 & 0.39 & 0.31 & 0.84 & 0.83 & 0.25 \\
\hline Pvrl2 & 1.79 & 3.84 & 0.77 & 4.65 & 1.56 & 1.58 \\
\hline
\end{tabular}




\begin{tabular}{|c|c|c|c|c|c|c|} 
Rae1 & 0.1 & 0.02 & 0.66 & 0.13 & 0 & 0.05 \\
\hline Tap1 & 129.22 & 603.45 & 127.02 & 0.25 & 0.11 & 43.17 \\
\hline Tgfb1 & 1.09 & 7.42 & 2.1 & 1.44 & 0.54 & 0.99 \\
\hline Tgfbr1 & 0.75 & 12.06 & 1.4 & 0.73 & 0.86 & 1.14 \\
\hline Tgfb2 & 0.37 & 1 & 1.35 & 0.41 & 0.64 & 0.33 \\
\hline Tgm 2 & 3.72 & 30.82 & 2.46 & 5.02 & 2.88 & 2.22 \\
\hline Tnf & 0.41 & 0.7 & 0.18 & 0.35 & 0.26 & 0.56 \\
\hline Trem 2 & 393.05 & 0.03 & $1.5 \times 10^{4}$ & 0.01 & 0 & 0.57 \\
\hline Ubc & 7.7 & 11.26 & 1.23 & 1.18 & 1.84 & 1.1 \\
\hline Ubd & 28.71 & 10.65 & 0.56 & 4.56 & 0.78 & 1.17 \\
\hline Ulbp1 & 2.08 & 6.53 & 0.38 & 1.37 & 0.47 & 1.04 \\
\hline
\end{tabular}

\subsection{Expression of genes in aGvHD rat compared to syngeneic rats in different tissues}

All fold changes for genes regulated in aGvHD rats compared to syngeneic rats have been listed in Table 7.4 for the different tissues. Fold changes $>1$ represent upregulation and $<1$ represent downregulation of the genes. The gene-tissue combinations for which the regulation was significant $(\mathrm{p}<0.05)$ are in bold and in blue. All genes are listed alphabetically.

Table 7.4 Fold changes of regulated genes in aGvHD rats compared to syngeneic rats in different tissues

\begin{tabular}{|c|c|c|c|c|}
\hline Genes & Liver & Lung & Skin & $\begin{array}{c}\text { Small } \\
\text { intestine }\end{array}$ \\
\hline C1qtnf7 & 0.4 & 1.06 & 1.36 & 0.68 \\
\hline Cd226 & 10.63 & 0.61 & 4.33 & 1.24 \\
\hline Hcls1 & $\mathbf{5 . 2 8}$ & 2.34 & 1.3 & 1.71 \\
\hline Htra1 & 0.77 & $\mathbf{0 . 5 3}$ & 0.54 & 0.85 \\
\hline Lgals7 & 26.12 & 1.19 & 0.61 & 2.06 \\
\hline Lilra5 & 20.51 & 3.87 & 0.63 & 1.59 \\
\hline Lst1 & 172.84 & 4.69 & 0.8 & 1.94 \\
\hline Msr1 & 8.54 & 0.86 & 0.89 & 1.08 \\
\hline Klrk1 & 94.31 & 1.11 & 1.95 & 0.7 \\
\hline Pik3ap1 & 3.01 & 1.06 & 0.61 & 3.33 \\
\hline Pstpip1 & 3.48 & $\mathbf{4 . 5 6}$ & 0.97 & 4.65 \\
\hline Ptger2 & $\mathbf{6 . 9 8}$ & 1.67 & 9.83 & 1.16 \\
\hline Ptpn7 & $\mathbf{3 . 6 7}$ & 2.02 & 4.39 & 1.2 \\
\hline Pvr & 0.43 & 0.77 & 0.58 & 1.27 \\
\hline Prrl2 & 1.42 & 0.76 & 2.21 & $\mathbf{2 . 8 7}$ \\
\hline Rae1l & $\mathbf{1 8 5 . 7 8}$ & 4.89 & $\mathbf{0 . 0 1}$ & 0.33 \\
\hline Rrlt & 15.75 & 0.27 & 0.1 & 0.23 \\
\hline Tap1 & $\mathbf{2 . 9 8}$ & 2.12 & 0.78 & 3.3 \\
\hline Tgm2 & 3.17 & 0.97 & 0.36 & 1.57 \\
\hline Trem2 & 1.59 & 4.65 & 0.54 & 2.84 \\
\hline Ubd & 80.44 & $\mathbf{5 8 . 5 8}$ & 96.29 & $\mathbf{3 0 . 8 5}$ \\
\hline
\end{tabular}




\section{Curriculum vitae}

\section{Pranali Nitin Shah}

Date of Birth: 09.07.1989

Born in Gujarat, India

\section{Address and contact details}

Department of Cellular and Molecular Immunology

Humboldtallee 34

37073 Göttingen

Lab: 0551/3913857

Email:pranali.04@gmail.com

\section{Education}

Aug 2013 - present $\quad$ PhD. thesis at Georg-August University Göttingen at the Department of Cellular and Molecular Immunology.

Thesis title: Identification of genes regulated in target organs of acute graft versus host disease in different species

Sept 2011 - Aug 2012 Master of Science in Infection and Immunity at University College London, UK.

Thesis title: Investigation of antigen dominance in serum antibody response to nasopharyngeal colonization in mice with Streptococcus pneumoniae

Aug 2007 - May 2011 Bachelor of Technology in Biotechnology at SRM University, Chennai, India.

Thesis title: Investigation of immunological and virological effects of antiretroviral therapy in HIV-TB co-infected individuals in South Indian populations 


\section{Publications}

Jalapothu D, Boieri M, Crossland RE, Shah P, Butt IA, Norden J, Dressel R, Dickinson AM and Inngjerdingen $M$ (2016). Tissue-specific expression patterns of microRNA during acute graftversus-host disease in the rat. Front. Immunol. 7:361.

Boieri M., Shah P., Dressel R., Inngjerdingen M. (2016). The role of animal models in the study of hematopoietic stem cell transplantation and GvHD: A historical overview. Front. Immunol. 7:333.

Isernhagen A., Schilling D., Monecke S., Shah P., Elsner L., Walter L., Multhoff G., Dressel R. (2016a). The MICA-129Met/Val dimorphism affects plasma membrane expression and shedding of the NKG2D ligand MICA. Immunogenetics 68, 109-123.

Isernhagen A., Malzahn D., Monecke S., Schilling D., Shah P., Multhoff G., Wulf G., Kube D., Bickeböller H., and Dressel R. (2016b). Functional consequences of genetic polymorphisms in the NKG2D receptor signaling pathway and putative gene interactions. Recept. Clin. Investig. 3.

\section{Congress}

Shah P, Jörß K, Fischer H, Zaitseva O, Monecke S, Hamann C, Walter L, Reichardt HM, Dressel $\mathrm{R}$ (2015) Gene expression profiling indicates an increased expression of the activating NK receptors NKG2D and DNAM-1 and their ligands during murine acute graft versus host disease. In: Abstracts of the Natural Killer Cell Symposium NK 2015, Göttingen. 07.10.2015 - 09.10.2015, p. 68 , poster

Shah P, Boieri M, Fischer H, Ghimire S, Jörß K, Zaitseva O, Hamann C, Monecke S, Walter L, Inngjerdingen M, Holler E, Reichardt HM, Dressel R (2016) Gene expression profiling indicates an increased expression of the activating NK receptors NKG2D and DNAM-1 and their ligands during acute graft versus host disease

In: Abstracts of Transplantation Immunology workshop, DGFI, Medical Hochschule Hannover 27.05.2016 - 28.05.2016, oral presentation 